\title{
A SPRAY REACTOR CONCEPT FOR CATALYTIC OXIDATION OF P-XYLENE TO PRODUCE HIGH-PURITY TEREPHTHALIC ACID
}

By

Meng Li

Submitted to the graduate degree program in Chemical \& Petroleum Engineering and the Graduate Faculty of the University of Kansas in partial fulfillment of the requirements for the degree of Doctor of Philosophy.

Bala Subramaniam (Chairperson)

Daryle H. Busch

$\begin{array}{r}\hline \text { Raghunath V. Chaudhari } \\ \hline \text { Peter D. Metelski } \\ \hline \text { Laurence R. Weatherley }\end{array}$

Date Defended: July 18, 2013 
The Dissertation Committee for Meng Li

certifies that this is the approved version of the following dissertation:

\section{A SPRAY REACTOR CONCEPT FOR CATALYTIC OXIDATION OF P-XYLENE TO PRODUCE HIGH-PURITY TEREPHTHALIC ACID}

Bala Subramaniam (Chairperson)

Date approved: July 20, 2013 


\begin{abstract}
Terephthalic acid (TPA), with current annual world capacity of exceeding 50 million metric tons, is a commercially important chemical used primarily in the manufacture of polyesters. A spray reactor in which the liquid phase, containing dissolved $p$-xylene $(p \mathrm{X})$ and the catalyst $(\mathrm{Co} / \mathrm{Mn} / \mathrm{Br})$, is dispersed as fine droplets by a nozzle into a continuous vapor phase containing the oxidant $\left(\mathrm{O}_{2}\right)$ is shown to produce high-purity TPA with less than 25 ppm 4carboxybenzaldehyde (4-CBA) in the solid TPA product. In sharp contrast, the solid TPA product obtained from a conventional stirred reactor similar to the configuration used in the conventional Mid-Century (MC) process contains nearly 1000 ppm 4-CBA even though the reactor is operated at similar pressure and temperature $\left(15\right.$ bar and $\left.200{ }^{\circ} \mathrm{C}\right)$ but with the gas phase dispersed into the liquid phase. The dramatic improvement in TPA product quality during spray reactor operation is attributed to two main factors: the alleviation of interphase gas-liquid mass transfer limitations that facilitates more complete oxidation of the $p \mathrm{X}$ and the intermediate oxidation products to TPA, and reduced backmixing that enhances the oxidation rates. Kinetic studies of $p \mathrm{X}$ oxidation to TPA performed in a well-stirred $50 \mathrm{~mL}$ reactor confirm that the intermediate oxidation steps are subject to mass transfer limitations even at the highest rpm used. Theoretical calculations show that the time constants for $\mathrm{O}_{2}$ diffusion in typical spray droplets (assumed to be $50 \mu \mathrm{m}$ diameter) are one to two orders of magnitude lower than the kinetic rate constant confirming complete $\mathrm{O}_{2}$ penetration and saturation of the droplets.
\end{abstract}

Gas phase concentration measurements show that in the spray reactor gas phase CO formation is roughly one-fourth of that in the MC process, indicative of solvent burning. This decrease is attributed to the shorter residence times in the spray reactor. Further, the usage of $\mathrm{CO}_{2}$ as an inert 
gas and the dominance of acetic acid $(>50 \mathrm{~mol} \%)$ in the vapor phase under reaction conditions create a gas phase environment that falls outside of the flammability envelope.

Mathematical modeling of the stirred reactor using MC process conditions accurately predicts the steady state temperatures observed in industrial reactors $\left(195^{\circ} \mathrm{C}\right)$. The model also clearly divulges that the cooling provided by partial evaporation of the acetic acid solvent, upon absorbing the heat of reaction at the set reactor pressure, is vital to maintain stable steady state operation. Experimental results clearly attest to the significance of reliable pressure control to prevent undesired temperature rises. Comparative economic analyses and gate-to-gate and cradle-to-gate life cycle assessments show that the spray process significantly reduces capital and operating costs by $55 \%$ and $16 \%$ respectively, and also imposes less adverse environmental impacts than the MC process. These benefits of the CEBC spray process are mainly derived from the non-requirement of the hydrogenation step required in the conventional process for purifying the crude TPA. Thus, the spray reactor concept has the potential to be a greener and more sustainable process for making polymer-grade dicarboxylic acids in one step. The results from this dissertation provide valuable guidance for the rational design and development of a continuous spray reactor. 


\section{Graphical Abstract}

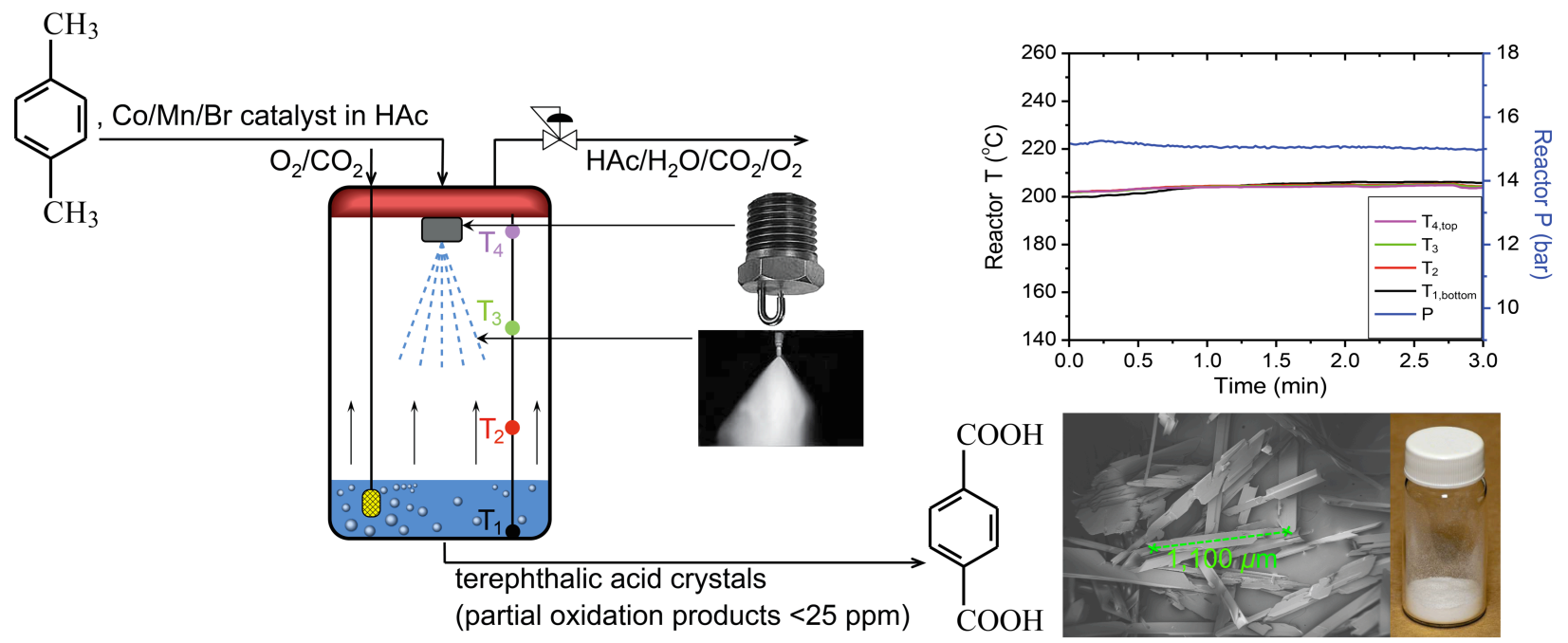


"The way ahead is long and has no ending; yet high and low I'll search with my will unbending."

\author{
- Qu Yuan \\ (Warring States Period of Ancient China)
}

Dedicated to my parents, Yuefang Hao and Zhicai Li, for thirty-one years of love and support, without which this work would not have been possible 


\section{Acknowledgements}

I would like to express my most sincere gratitude and appreciation to my advisor, Prof. Bala Subramaniam, for his guidance and support throughout my graduate study. Prof. Subramaniam provided me with the great opportunity to work on this project, and offered excellent technical and professional advice whenever I had difficulties in my research. This project has long captured my fascination and imagination, including chemical reactor design and operation, catalysis and kinetics, and chemical process simulation and modeling. It was Prof. Subramaniam's encouragement and support that sustained my interest. In addition to his guidance on my dissertation work, Prof. Subramaniam has taught me a lot of things. He has taught me about critical thinking, about developing an idea, about analyzing and solving problems, about perseverance and staying positive when research hits a snag. Prof. Subramaniam has been and will always be a great mentor and an invaluable asset to me.

Special thanks are also due to Prof. Daryle Busch, Prof. Raghunath Chaudhari, Dr. Peter Metelski and Prof. Laurence Weatherley for serving on my PhD committee, and for providing valuable instruction and guidance throughout my time in the program.

I would like to acknowledge and thank Dr. Fenghui Niu and Dr. Xiaobin Zuo, for their generous assistance in experiments. Without their hard work and input I would not have been able to complete this dissertation to my satisfaction. I would like to thank Dr. Todd Williams and Dr. Robert Drake for their help with the analytical methods. Thanks are also due to Mr. Edwin Atchison and Mr. Thomas Ruddy for their valuable input to the equipment design and economic and environmental impact analyses.

In addition, I would like to thank my colleagues at CEBC (past and present): Dr. Darryl Fahey, Dr. Chris Lyon, Dr. Claudia Bode, Mr. James Busse, Ms. Deanna Bieberly, Ms. Nancy 
Crisp, Ms. Rhonda Partridge, Ms. Megan Gannon, Mr. Alan Walker, Ms. Rebecca Meyer, Dr. William Kirk Snavely, Dr. Jing Fang, Dr. Jackson Ford, Dr. Andrew Danby, Dr. Michael Lundin, Dr. Hyun-Jin Lee, Dr. Debdut Roy, Dr. Suparna Roy, Dr. Juan Bravo, Dr. Bibhas Sarkar, Dr. Haijun Wan, Dr. Swarup Maiti, Dr. Ramanathan Anand, Dr. Maheswari Rajamanickam, Dr. Amit Chaudhari, Dr. Shengwei Tang, Dr. Chad Johnson, Dr. Kening Gong, Dr. Wei Ren, Dr. Azita Ahosseini, Dr. Sylvia Johnson, Dr. Madhav Ghanta, Dr. Zhuanzhuan Xie, Ms. Arely Torres, Mr. Faisal Baksh, Ms. Yuanchun Li, Mr. Xin Jin, Ms. Wenjuan Yan, Ms. Xiaohui Hu, Mr. Steve Tang, Ms. Qing Pan, Ms. Xuchao Li, Mr. Dupeng Liu, and Mr. He for their friendship and help.

In short, I am grateful to all of the above incredible people I met during this journey who contributed to my academic and personal development.

The majority of the research in this dissertation was funded by National Science Foundation and U.S. Department of Agriculture.

Finally, I own so much to my parents, Yuefang Hao and Zhicai Li, for their unconditional love and support, and for respecting my decision to go to another country for my continued education. I have to compensate for not being with them for all these years and find a balance between career and family, which will be another life project of mine. I would also like to thank my aunt Junyue Hao and my uncle-in-law Huanbing Zhai, for their continued care and support. 


\section{Table of Contents}

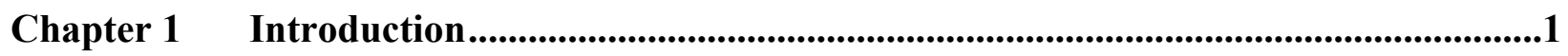

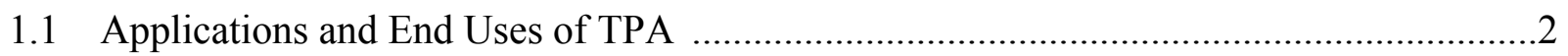

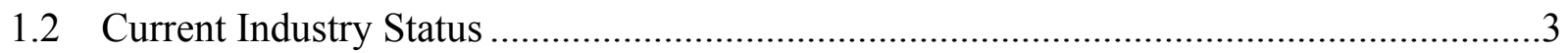

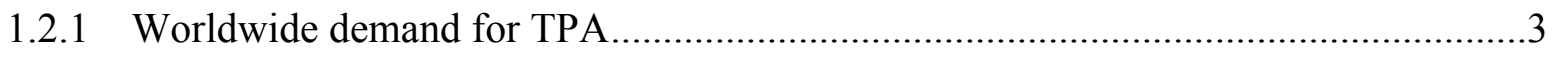

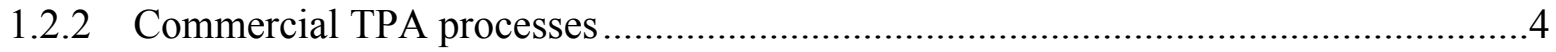

1.3 Technical Aspects of TPA Process .......................................................................

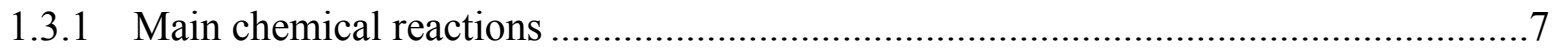

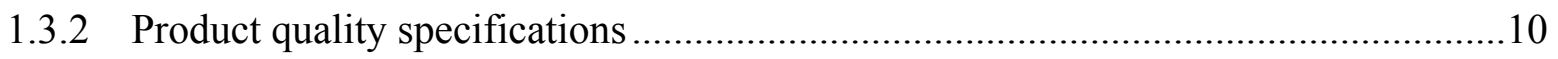

1.3.3 Mechanism of $p \mathrm{X}$ oxidation with $\mathrm{MC}$ catalyst ............................................. 11

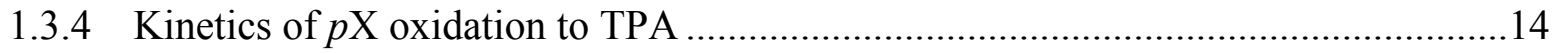

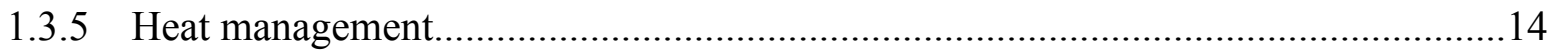

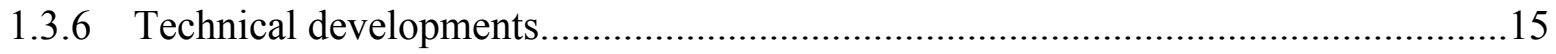

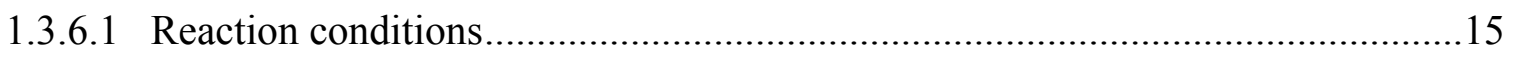

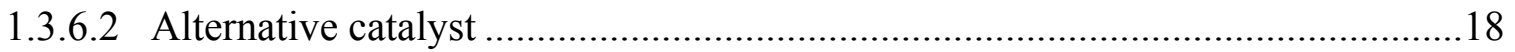

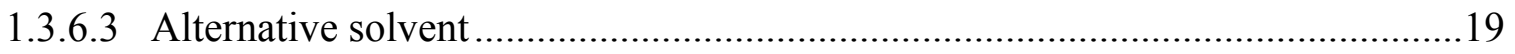

1.4 Challenges in the MC Process ..........................................................................2

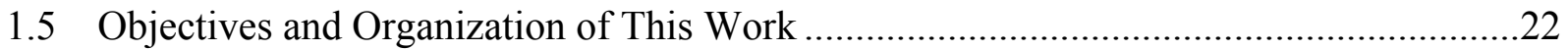

\section{Chapter 2 Kinetic Studies of Homogeneous p-Xylene Oxidation to Terephthalic Acid in Acetic Acid with a Co/Mn/Br Catalyst................................................................24}

2.1 Solubility Studies of the MC Process Related Components .........................................24 


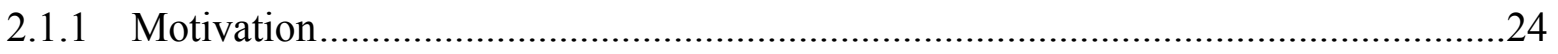

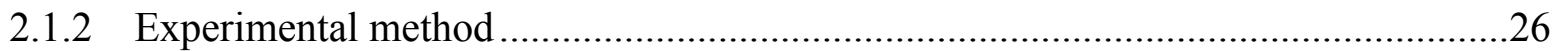

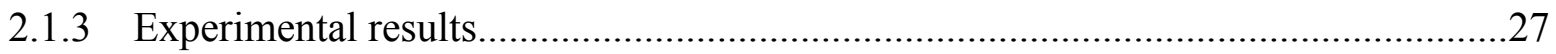

2.2 Previous Work on Kinetics of $p X$ Oxidation to TPA ……..............................................29

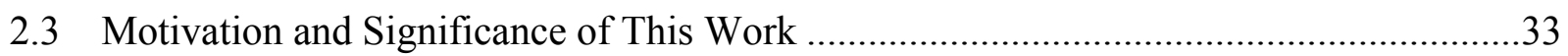

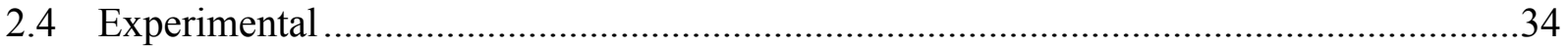

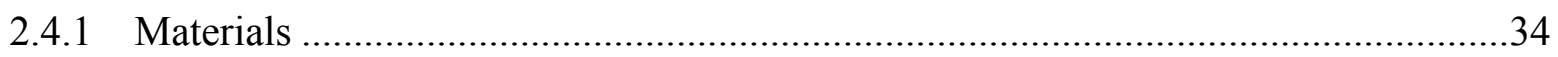

2.4.2 Experimental setup and procedure ........................................................................34

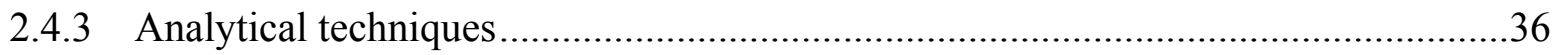

2.5 Lumped Kinetic Scheme and Semi-Batch Reactor Model ...............................................37

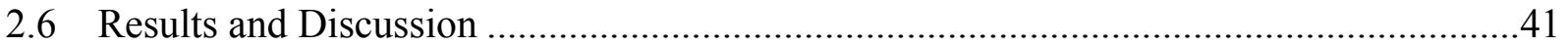

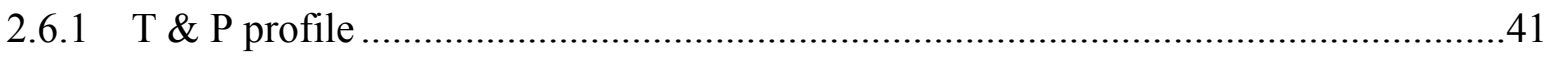

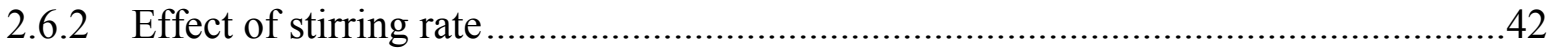

2.6.3 Comparison of estimated rate constants with literature data .....................................44

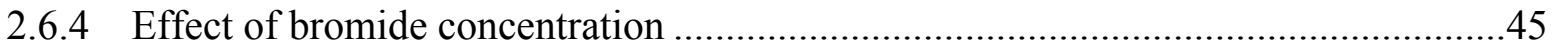

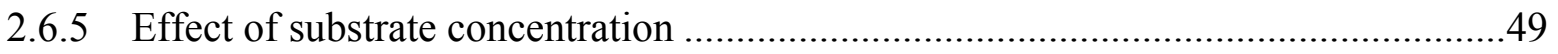

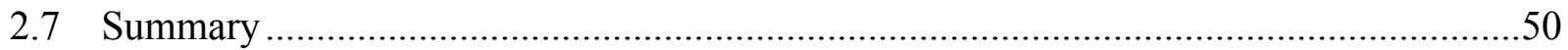

\section{Chapter 3 A Spray Reactor Concept for Catalytic Oxidation of $p$-Xylene to Produce}

High-Purity Terephthalic Acid .....................................................................................52

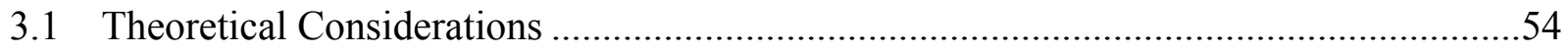

3.1.1 Rationale for choosing spray reactor as an alternative to a stirred reactor .................54

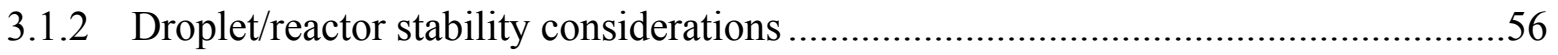

3.1.3 Vapor phase flammability considerations.............................................................61 
3.2 Experimental Demonstration

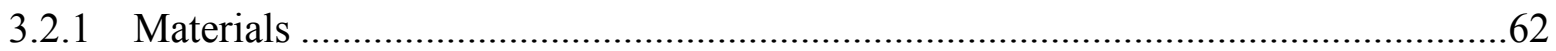

3.2.2 Oxidation experiments in spray reactor ........................................................63

3.2.3 Benchmark oxidation experiments in a stirred reactor .......................................65

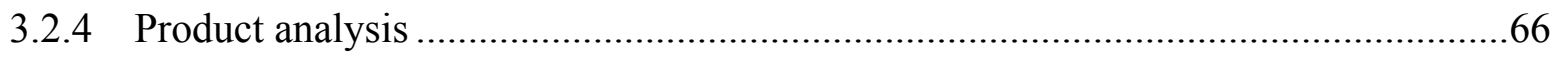

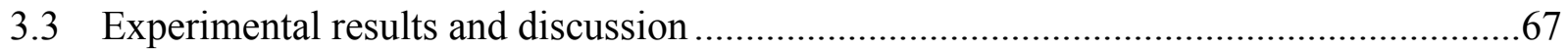

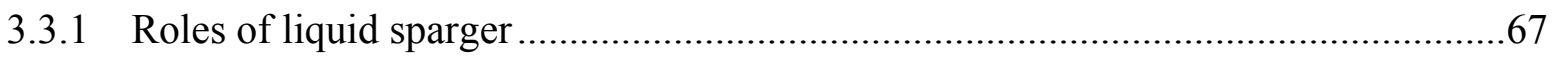

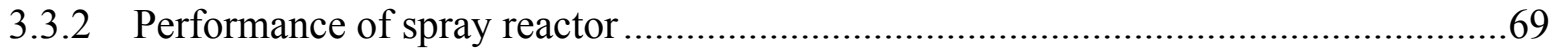

3.3.3 Comparison of product from spray and stirred reactors .....................................74

3.3.4 CO formation as a measure of burning reactions............................................. 77

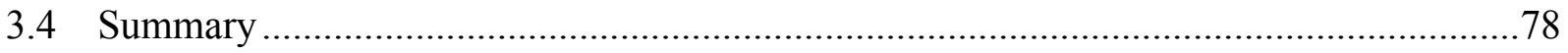

\section{Chapter 4 Development of A Continuous Spray Process for Terephthalic Acid}

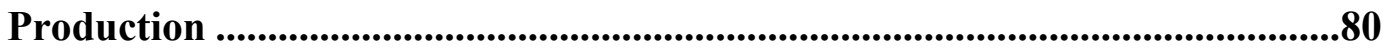

4.1 Continuous Spray Reactor: Initial Efforts............................................................. 80

4.2 Preliminary Experimental Results from Continuous operation of the 4 L Spray Reactor

4.3 Summary

Chapter 5 Economic and Environmental Impact Assessment of the Spray Process for Terephthalic Acid Production .....................................................................................88

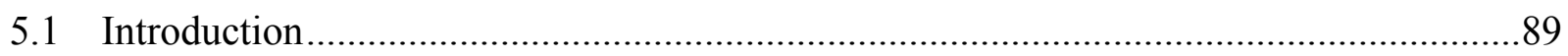

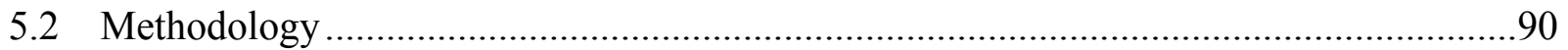

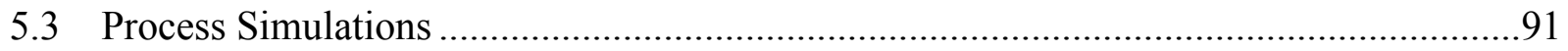




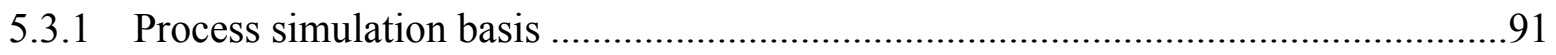

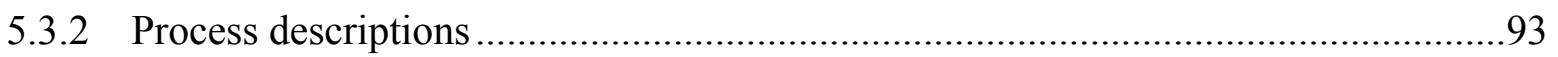

5.3.2.1 Conventional MC process ..............................................................................96

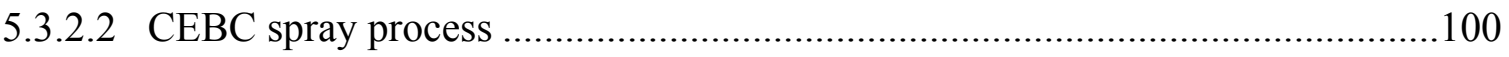

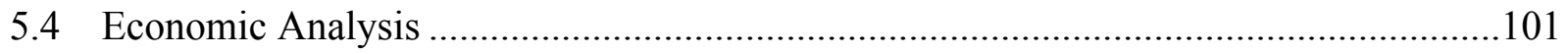

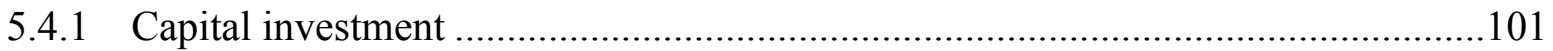

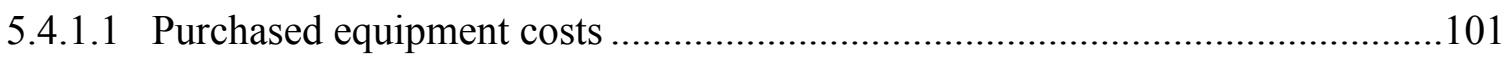

5.4.1.2 Estimate of capital investment ......................................................................102

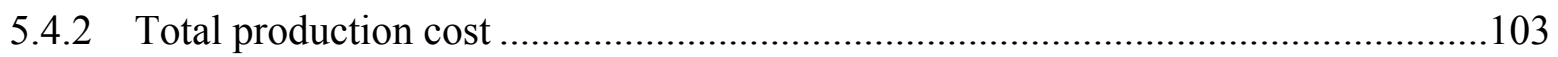

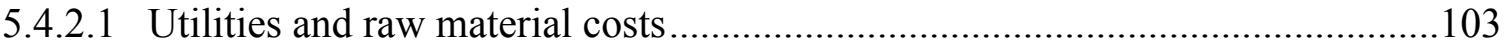

5.4.2.2 Estimate of total production cost .................................................................106

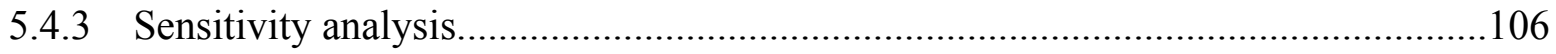

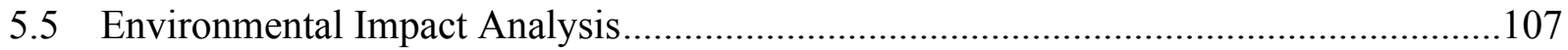

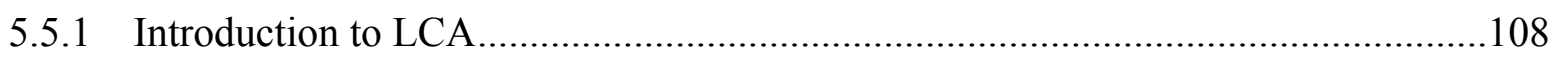

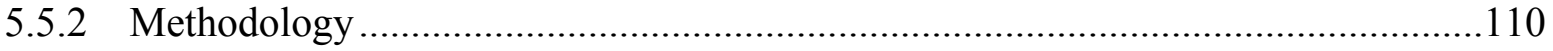

5.5.3 Environmental impact analysis of TPA processes................................................112

5.5.3.1 Goal and scope definition .....................................................................112

5.5.3.2 Life cycle inventory ...............................................................................117

5.5.3.3 Life cycle impact assessment......................................................................119

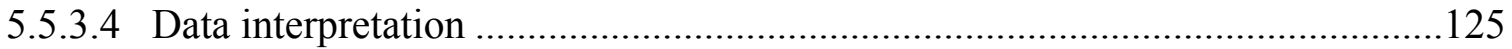

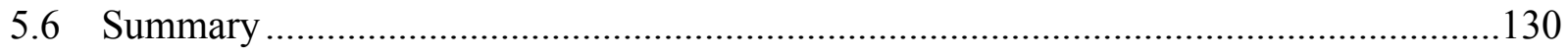

Chapter 6 Conclusions and Recommendations..................................................................132 


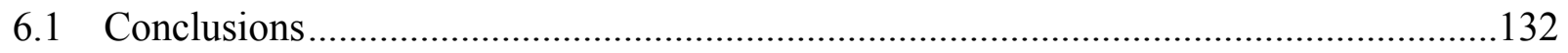

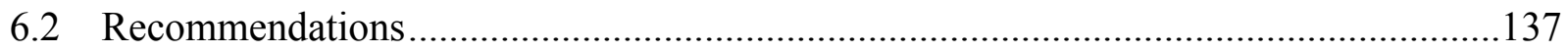

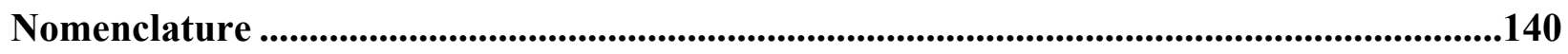

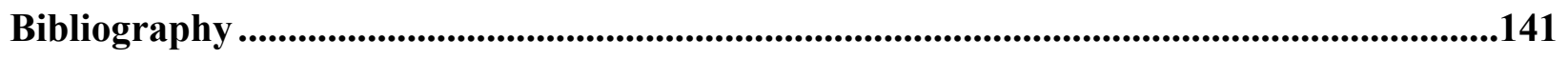

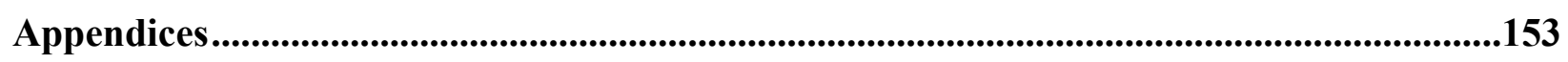

Appendix A: Numerical Data of the Experimental Solubilities .......................................153

Appendix B: HYSYS Simulation for Saturated Acetic Acid ........................................154

Appendix C: HYSYS Simulation of MC and CEBC Processes ......................................155

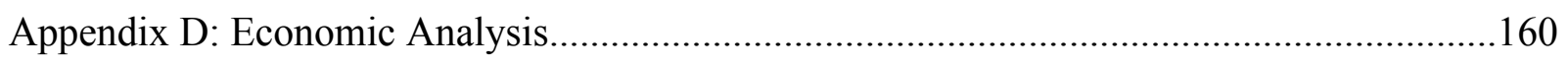

Appendix E: Environmental Impact Analysis ..........................................................182 


\section{List of Tables}

Table 1.1 Oxidation reaction conditions and technology characteristics of major commercial

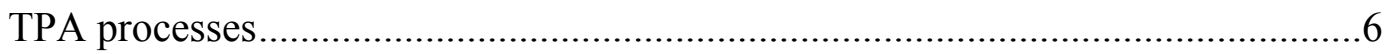

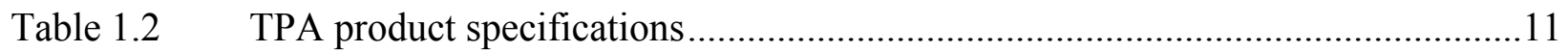

Table 2.1 Temperature ranges of available literature solubility data...............................26

Table 2.2 Various kinetic models for $p \mathrm{X}$ oxidation to TPA from literature.........................31

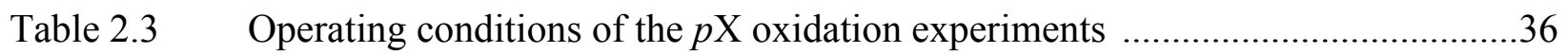

Table 2.4 Estimated reaction rate constants at different stirring rates (initial $[p \mathrm{X}]=0.025 \mathrm{M}$; all other experimental conditions are as shown in Table 2.3) ...........................44

Table 2.5 Comparison of estimated rate constants with literature data .............................45

Table 2.6 Estimated reaction rate constants at different bromide concentrations (initial $[p X]$ $=0.025 \mathrm{M}$; stirring rate $=1000 \mathrm{rpm}$; all other experimental conditions are as shown in Table 2.3) ............................................................................4

Table 2.7 Estimated reaction rate constants at different $p \mathrm{X}$ concentrations $(\mathrm{P}=15$ bar; $\mathrm{T}=$ $200\left({ }^{\circ} \mathrm{C}\right.$; stirring rate $=1000 \mathrm{rpm}$; all other experimental conditions are as shown

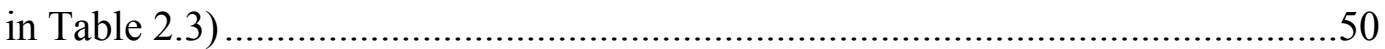

Table 3.1 Estimation of Hatta number for simultaneous $\mathrm{O}_{2}$ diffusion and reaction in a

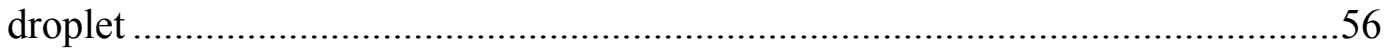

Table $3.2 \quad$ Purity of solid TPA product obtained from the spray reactor ..........................69

Table $3.3 \quad$ Product specifications from run \#1 of Table 3.2 ...............................................73

Table 3.4 Comparison of product purity and yields from spray and stirred reactors ..........74

Table 3.5 $\quad \mathrm{CO}$ analysis during the $p \mathrm{X}$ oxidation in the spray reactor .................................77 
Table 4.1 Gas sparger effects on solid TPA product quality from the 4-L spray reactor .....82

Table 4.2 Acetic acid amounts from the top (gas stream) and bottom (liquid stream) of the

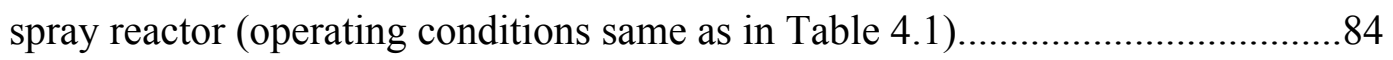

Table 4.3 Solid TPA product quality during 25-min continuous run in $4 \mathrm{~L}$ spray reactor ....86

Table 5.1 Key operating parameters for the simulation of the MC and CEBC spray processes .92

Table 5.2 Assumed acetic acid use and loss for the MC process and the CEBC spray process

Table 5.3 Comparison of capital costs for both MC and spray processes

Table 5.4 Utilities and raw material costs for both processes.

Table 5.5 Comparison of conventional MC process VOC emissions obtained from U.S. EPA Toxic Release Inventory (TRI) data for commercial facility with $\mathrm{GaBi}^{\circledR}$ estimated emissions 119

Table 5.6 Comparison of conventional MC process $\mathrm{CO} 2$ emissions obtained from U.S. EPA GHG data for commercial facility with $\mathrm{GaBi}^{\circledR}{ }^{\circledR}$-estimated emissions .120

Table A1 Experimental solubilities of TPA, 4-CBA and $p$-TA in acetic acid or aqueous acetic acid 153

Table C1 Simulation parameters for the oxidation section of both processes. 155 Table C2 Simulation parameters for the hydrogenation section of conventional MC process

Table D1 Purchased equipment cost estimation details of conventional MC process ........160

Table D2 Purchased equipment cost estimation details of CEBC spray process ...............175

Table D3 Total production cost for both processes ............................................... 181 
Table E1 Predicted gate-to-gate environmental impact potentials for conventional and CEBC processes.

Table E2 Predicted cradle-to-gate environmental impact potentials for conventional and

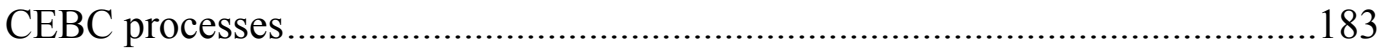




\section{List of Figures}

Figure 1.1 Major applications of polyethylene terephthalate (PET) ....................................

Figure 1.2 Projected growth in demand for TPA in various geographic regions [6] f.............4

Figure 1.3 Sequential reaction network for $p \mathrm{X}$ oxidation to TPA .....................................

Figure 1.4 Structures of major impurities formed during $p \mathrm{X}$ oxidation to TPA....................9

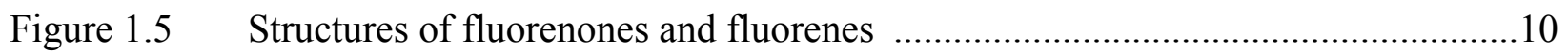

Figure 1.6 Model for redox activity of MC catalyst components [6]...............................12

Figure 1.7 Initiation, propagation and termination steps of free radical chain reactions during

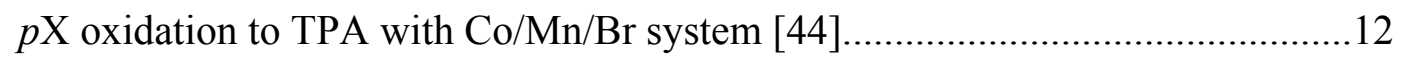

Figure 2.1 Typical T \& P profiles during solubility studies ( T \& P rise and fall due to stirrer

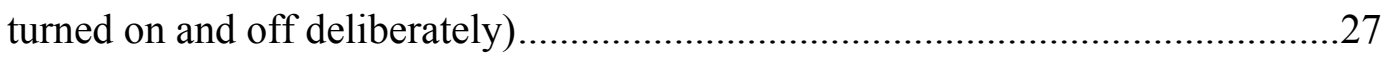

Figure 2.2 Solubilities of the MC process related components (14.5-15.5 bar in $\mathrm{N}_{2}$ ): (a) TPA;

(b) 4-CBA and $p$-TA .28

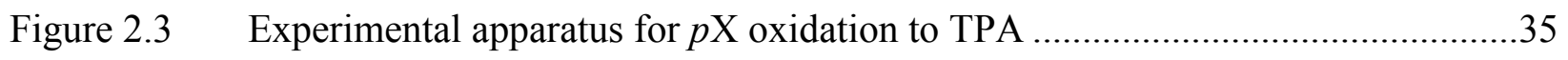

Figure 2.4 Typical HPLC chromatogram of a product sample (after $15 \mathrm{sec}$. of $p \mathrm{X}$ oxidation

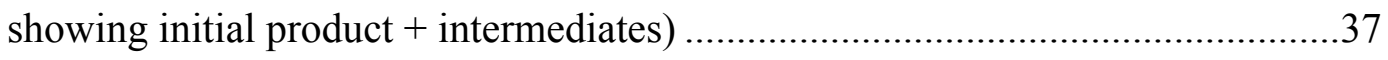

Figure 2.5 Proposed lumped kinetic scheme for $p \mathrm{X}$ oxidation to TPA ...............................38

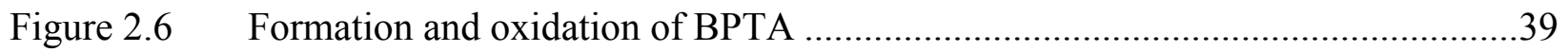

Figure 2.7 Temporal temperature and pressure profiles during intrinsic kinetic studies of $p \mathrm{X}$ oxidation in a stirred reactor .41 
Figure 2.8 Experimental and simulated product distributions at different stirring rates (Reaction conditions: $\mathrm{T}=200^{\circ} \mathrm{C}, \mathrm{P}=15$ bar; Initial $p \mathrm{X}=25 \mathrm{mM}, \mathrm{Co}=12.5 \mathrm{mM}$, $\left.\mathrm{Mn}=12.5 \mathrm{mM}, \mathrm{Br}=32.5 \mathrm{mM} ; \mathrm{O}_{2}: \mathrm{CO}_{2}(\mathrm{~mol}: \mathrm{mol})=1: 1\right)$

Figure 2.9 Figure 2.9: Color changes of the reaction solution due to the formation of various cobalt bromides [104] at various bromide concentrations in acetic acid. $[\mathrm{Co}(\mathrm{II})]=$ $12.5 \mathrm{mM}$ .46

Figure 2.10 Experimental and simulated product distributions with various bromide concentrations (initial $[p \mathrm{X}]=0.025 \mathrm{M}$; stirring rate $=1000 \mathrm{rpm}$; all other experimental conditions are as shown in Table 2.3). .48

Figure 2.11 Proposed Scheme for the autoxidation of benzyl bromide [105] .........................49

Figure 2.12 Effect of initial concentration of $p \mathrm{X}$ on the reaction rate [99]............................50

Figure $3.1 \quad$ Factors that dictate reactor performance [109] .................................................52

Figure 3.2 Variation of acetic acid boiling point and heat of vaporization with pressure .......57

Figure 3.3 Schematic of oxidation reactor at typical MC process conditions $[13,14,20] \ldots . .58$

Figure 3.4 Predicted heat generation $\left(Q_{\mathrm{G}}\right)$ and heat removal $\left(Q_{\mathrm{R}}\right)$ rates for $p \mathrm{X}$ oxidation reactor (conditions noted in Figure 3.3)

Figure 3.5 Effects of water content on reactor temperature in MC process.. 61

Figure 3.6 Schematic of the spray reactor: The four thermocouple locations (black dots) are evenly spaced and numbered from the bottom to the top as follows: $\mathrm{T}_{1, \mathrm{bottom}}$ (in the liquid phase), $\mathrm{T} 2$ (above $\mathrm{T}_{1, \text { bottom }}$ ), $\mathrm{T} 3$ (above $\mathrm{T}_{2}$ ) and $\mathrm{T} 4$,top (above $\mathrm{T}_{3}$ ) .64

Figure 3.7 Schematic of the liquid sparger. .68

Figure 3.8 Measurement of acetic acid condensed from gas phase during black run (Left: without sparger; Right: with sparger) .68 
Figure 3.9 SEM (scanning electron microscope) image of TPA crystals produced from the spray reactor .70

Figure 3.10 Temporal temperature and pressure profiles during spray reactor operation: (a) with gas outlet (operating conditions correspond to run 1, Table 3.2); (b) without gas outlet (operating conditions correspond to run 4, Table 3.2); (c) with gas outlet (operating conditions correspond to run 3, Table 3.2). .72

Figure 3.11 Temperature profiles during the cool down phase: (a) stirred reactor; (b) spray reactor .76

Figure 4.1 Dimensions of $700 \mathrm{~mL}$ and $4 \mathrm{~L}$ spray reactors .81

Figure 4.2 T \& $\mathrm{P}$ profiles during a 25-min blank run with manual control of micro-metering valve .85

Figure 4.3 30-min blank run with stepper motor controlled micro-metering valve...............86

Figure 5.1 Process flow diagram for the conventional MC process ..................................94

Figure 5.2 Process flow diagram for the CEBC spray process .......................................95

Figure 5.3 Comparison of purchased equipment costs for MC and spray processes ...........102

Figure 5.4 Comparison of total production costs for both processes (Others include other direct, indirect costs and general expenses such as labor, research, plant overhead, operating supplies and maintenance, details are provided in Table D3 of Appendix D) 106

Figure 5.5 Capital and operating cost rise with acetic acid throughput in CEBC spray process 107

Figure 5.6 Methodological framework for LCA of TPA processes. .110 
Figure 5.7 System boundaries for gate-to-gate and cradle-to-gate LCA: (a) conventional MC

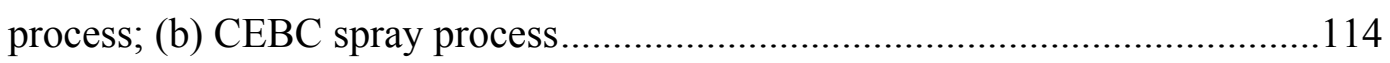

Figure 5.8 Flow model for cradle-to-gate LCA simulated in $\mathrm{GaBi}^{\circledR}$ : (a) conventional MC process; (b) CEBC spray process ..................................................................118

Figure 5.9 Comparison of predicted gate-to-gate environmental impact potential for both processes (Orange: MC process oxidation section; Magenta: MC process hydrogenation section; Cyan: spray process; Shaded: environmental impact potential of direct process emissions such as off-gas, wastewater, VOCs from unit operations and fugitive emissions; Unshaded: fuel combustion for process steam production)

Figure 5.10 Predicted cradle-to-gate environmental impact potential for both processes (Orange: MC process; Cyan: Spray process) .......................................................124

Figure B1 HYSYS simulation of saturated acetic acid flow in the vapor phase .154 


\section{Chapter 1 Introduction}

The concepts of green chemistry and green engineering [1] were introduced nearly two decades ago in an effort to simultaneously achieve the "triple bottom line" — sustainability in economic, social, and environmental performance. By providing qualitative guidelines for the design of chemical products and processes that reduce or eliminate the use and generation of hazardous substances, the twelve principles of green chemistry and the twelve principles of green engineering [2] promote sustainability. As part of their professional responsibilities, chemists and chemical engineers should continue to evaluate alternative products and manufacturing processes, or retrofit existing procedures to meet customer needs, to maintain process safety and to enhance environmental protection while ensuring economic viability.

The University of Kansas Center for Environmentally Beneficial Catalysis (CEBC) implements its mission guided by the principles of green chemistry and green engineering to develop sustainable chemical technologies in diverse areas such as selective oxidation, hydroformylation, alkylation and hydrogenation. Sustainable technologies are achieved by designing highly active and selective catalysts, employing benign media and process intensification via novel reactor types. As part of the selective oxidation testbed, $p$-xylene $(p \mathrm{X})$ oxidation to terephthalic acid (TPA) has been investigated extensively at CEBC in an effort to develop greener alternatives to the conventional Mid-Century process. One of the key published findings to date is that the use of a $\mathrm{CO}_{2} / \mathrm{O}_{2}$ mixture instead of the traditional air oxidation system effectively reduces the solvent burning rate [3]. 
This dissertation research complements previous work by CEBC researchers and addresses some of the sustainability challenges associated with industrial processes for TPA production. In this chapter, the state-of-the-art of TPA technologies is reviewed.

\subsection{Applications and End Uses of TPA}

TPA is a commercially important aromatic compound used mainly as a major precursor of polyethylene terephthalate (PET) polymer, which is produced by polycondensation of ethylene glycol with TPA. More than $90 \%$ of the worldwide production of TPA is used to make PET, consumed primarily for the manufacture of polyester fibers, solid-state resins (also known as bottle-grade resins) and polyester film.

Figure 1.1 shows the major applications of PET. Polyester fiber application accounts for a majority of TPA consumption. End products of polyester fibers include industrial filaments and polyester filament yarns for consumer products such as apparel, carpets, home textiles and fiberfill. PET solid-state resins are the next major market for TPA. The end-use applications are

for packaging and plastic bottles as food and beverage containers. The third major TPA application is polyester film mostly used for magnetic tape and packaging. Remaining minor TPA uses include production of polybutylene terephthalate, polytrimethylene terephthalate and aramid fibers. 


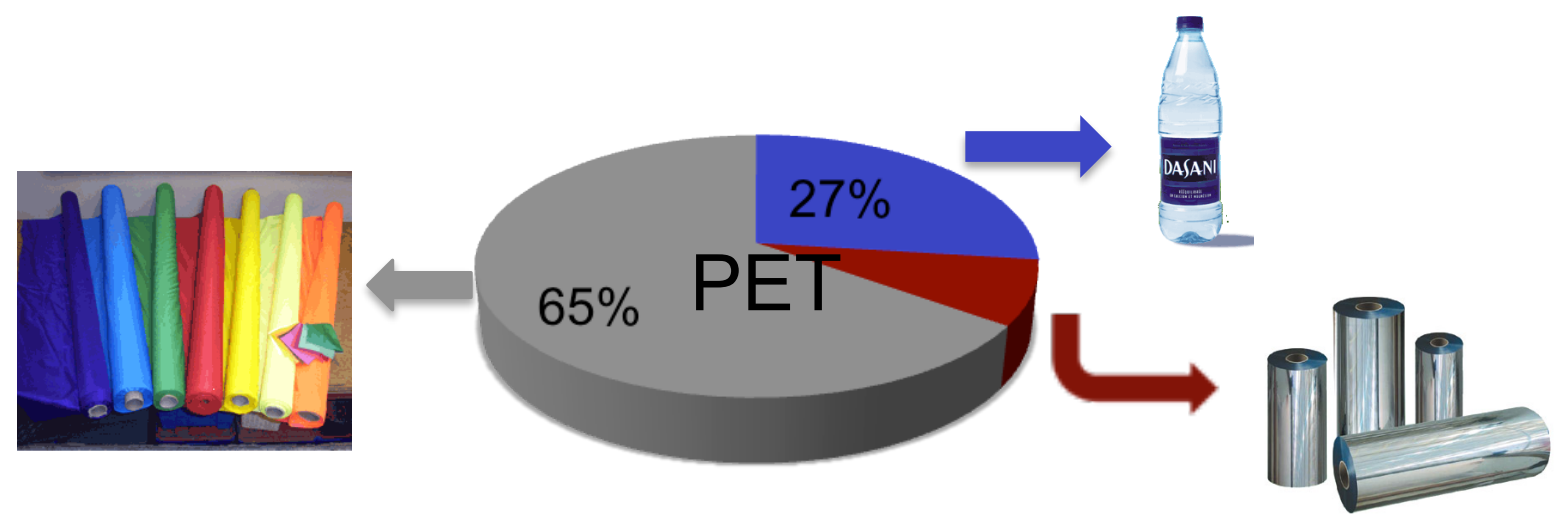

Figure 1.1: Major applications of polyethylene terephthalate (PET)

\subsection{Current Industry Status}

\subsubsection{Worldwide demand for TPA}

TPA is a growing industry worldwide and the demand is driven by the polyester markets. Global TPA supply and demand was approximately 42 million metric tons in 2009 [4]. From 1999 to 2009 , the global TPA demand grew at a rate of $7-8 \% / y r$. This growth rate decreased following the global economic recession in 2008 .

The current global TPA growth is largely driven by the Asia region, which accounts for about $70 \%$ of the world TPA capacity. Asian TPA market is primarily driven by PET fibers, while in North America and Western Europe, TPA growth is associated primarily with PET bottle resin market [5]. The TPA demand by geographic regions is shown in Figure 1.2 [6]. 


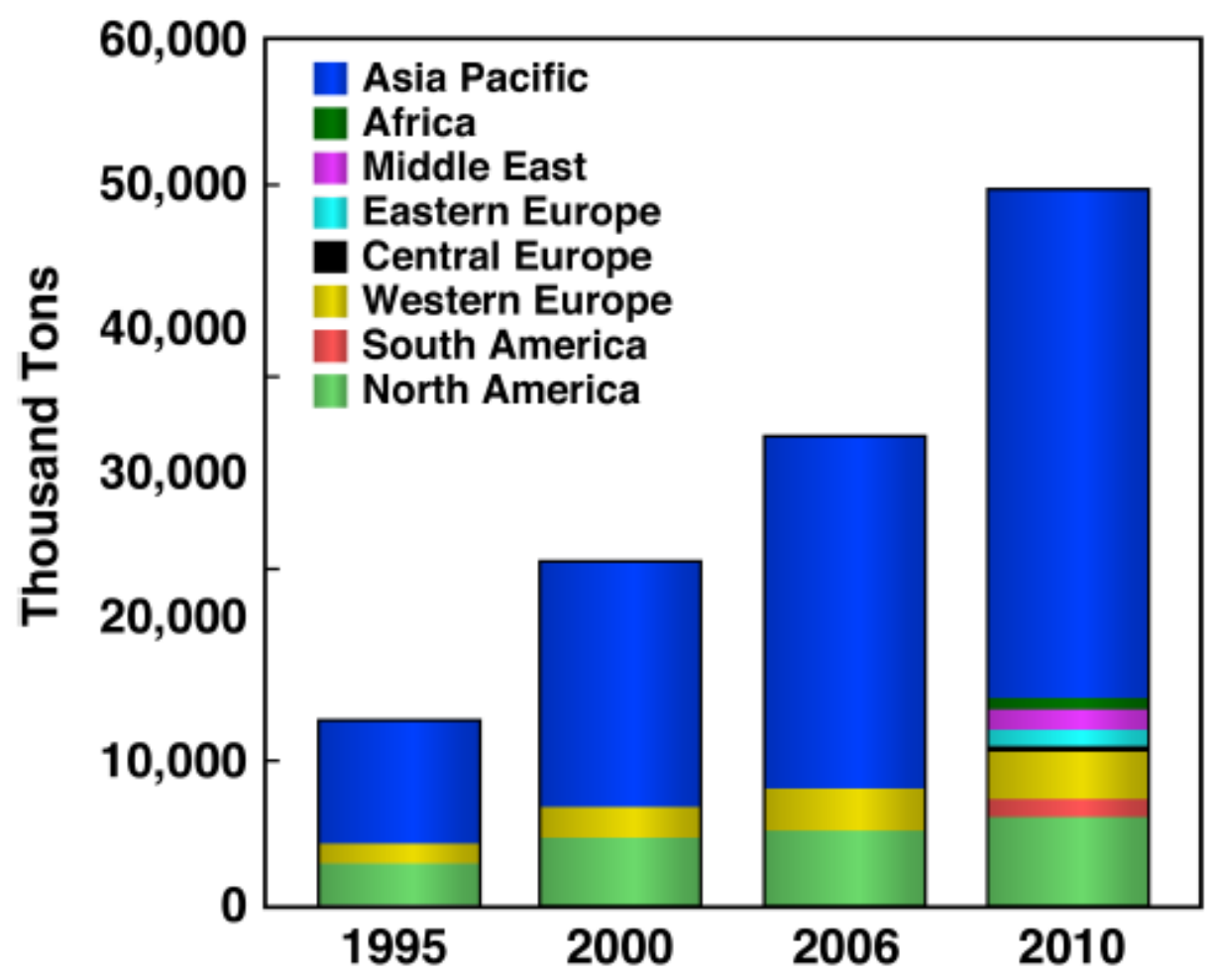

Figure 1.2: Projected growth in demand for TPA in various geographic regions [6]

\subsubsection{Commercial TPA processes}

Most of the commercial TPA processes are based on the core technology originally developed by Mid-Century Corporation - a liquid phase bromine-promoted catalytic oxidation of $p$-xylene $(p \mathrm{X})$ by means of air or molecular oxygen [7]. Less than $1 \%$ of the world capacity of TPA production is based on the DMT (dimethyl terephthalate) hydrolysis technology. Based on the impurity levels of the TPA product, the commercial TPA processes are categorized into Purified TPA (PTA) process and Medium Quality TPA (MTA) process.

The well-known Amoco (acquired by BP in 1997) Mid-Century (MC) process is currently the leading TPA technology. It involves an oxidation reactor system in which air is dispersed into the stirred liquid phase containing $p \mathrm{X}$ and $\mathrm{Co} / \mathrm{Mn} / \mathrm{Br}$ based catalyst dissolved in aqueous acetic acid. The reactors are lined with titanium to withstand the highly corrosive hydrobromic acid in 
the reaction mixture. A downstream hydrogenation reactor is deployed to remove 4carboxybenzaldehyde (4-CBA), the main and most troublesome impurity. The crude TPA solid from the oxidation reactor contains $1000-4000 \mathrm{ppm}$ of 4-CBA [8] due to incomplete oxidation. Most polyester applications require stringent TPA purity specifications of less than 25 ppm 4CBA and 150 ppm $p$-toluic acid ( $p$-TA) each [9] because 4-CBA and $p$-TA are polymerization terminators in the polymerization process for PET production. The erstwhile Amoco Company developed the hydrogenation purification step to upgrade the resulting crude TPA to high purity polymer-grade TPA or purified TPA (PTA) [10]. BP, DuPont, Dow Chemical, Mitsubishi Chemical, Eastman Chemical, Mitsui Chemicals, Interquisa, Hitachi and Grupo Petromex are currently the main commercial TPA technology license holders and licensors. BP is currently the world's largest TPA producer with an annual capacity of 7.5 million metric tons in 2011 [11].

By eliminating the hydrogenation step and adding one or more post-oxidation reactors, a Medium Quality Terephthalic Acid (MTA) process has been developed and commercialized [12]. Eastman Chemical is the original licensor of this technology. The TPA product from the MTA process has higher 4-CBA levels ( $\sim 500 \mathrm{ppm})$ compared to the MC process, which prevents its use in some polyester applications.

Generally, the variations of the MC process technology are related to reaction conditions, catalysts, off-gas treatment method, energy recovery method, acetic acid separation and recycling, and solid-liquid separation techniques. Table 1.1 summarizes the oxidation reaction conditions and technology characteristics of major commercial TPA processes assembled from a large number of patents. 
Table 1.1: Oxidation reaction conditions and technology characteristics of major commercial TPA processes

\begin{tabular}{|c|c|c|c|}
\hline Process & $\begin{array}{l}\text { Oxidation reaction } \\
\text { conditions }\end{array}$ & Technology characteristics & References \\
\hline $\begin{array}{l}\text { MC Process } \\
\text { (BP Amoco) }\end{array}$ & $\begin{array}{l}\mathrm{T}: 190-205^{\circ} \mathrm{C} \\
\mathrm{P}: 15-30 \mathrm{bar} \\
\text { catalyst: } \mathrm{Co} / \mathrm{Mn} / \mathrm{Br} \\
\text { solvent: acetic acid }\end{array}$ & $\begin{array}{l}\text { Leading TPA technology; } \\
\text { New-generation TPA process ( } X \\
\text { Technology): involves improvements in } \\
\text { water recycle and solid-liquid separation }\end{array}$ & $7,13-21$ \\
\hline $\begin{array}{c}\text { INVISTA } \\
\text { Process } \\
\text { (ICI-DuPont) }\end{array}$ & MC Process conditions & $\begin{array}{l}\text { TOUGH Max }{ }^{\mathrm{TM}} \text { process: involves improved } \\
\text { plant layout and solid-liquid separation } \\
\text { methods; } \\
\mathrm{R}^{2} \mathrm{R} \text { technology of residue recovery }\end{array}$ & $8,22-26$ \\
\hline $\begin{array}{c}\text { Dow-Inca } \\
\text { Process }\end{array}$ & MC Process conditions & $\begin{array}{l}\text { COMPRESS TM PTA technology: involves } \\
\text { pressure filtration technology and a novel } \\
\text { agitation system (to increase mass transfer } \\
\text { coefficient) }\end{array}$ & $27-29$ \\
\hline $\begin{array}{l}\text { Eastman- } \\
\text { Kodak } \\
\text { Process }\end{array}$ & $\begin{array}{l}\mathrm{T}: 120-175^{\circ} \mathrm{C} \\
\text { P: } 7.5-15 \text { bar } \\
\text { catalyst: Co/acetaldehyde } \\
\text { solvent: acetic acid }\end{array}$ & $\begin{array}{l}\text { Acetic acid is a co-product in the process; } \\
\text { Use of acetaldehyde in place of bromide } \\
\text { allows the use of conventional material of } \\
\text { construction such as stainless steel and } \\
\text { carbon steel; } \\
\text { Product purity } \sim 99 \% \text {. }\end{array}$ & $13,14,30,31$ \\
\hline $\begin{array}{l}\text { Toray } \\
\text { Process }\end{array}$ & $\begin{array}{l}\text { T: } 110-140{ }^{\circ} \mathrm{C} \\
\text { P: } 30 \text { bar } \\
\text { catalyst: Co/paraldehyde } \\
\text { solvent: acetic acid }\end{array}$ & $\begin{array}{l}\text { Paraldehyde is effective at relatively mild } \\
\text { reaction conditions resulting in less colored } \\
\text { impurities; } \\
\text { Use of acetaldehyde in place of bromide } \\
\text { allows the use of conventional material of } \\
\text { construction such as stainless steel and } \\
\text { carbon steel; } \\
\text { Product purity } ~ 99 \% \text {. }\end{array}$ & $13,14,32,33$ \\
\hline $\begin{array}{l}\text { Teijin } \\
\text { Process }\end{array}$ & $\begin{array}{l}\text { T: } 100-130{ }^{\circ} \mathrm{C} \\
\text { P: } 10 \text { bar } \\
\text { catalyst: Co } \\
\text { solvent: acetic acid }\end{array}$ & $\begin{array}{l}\text { High concentrations of Co are used under } \\
\text { mild conditions with no promotors; } \\
\text { Conventional material of construction such } \\
\text { as stainless steel and carbon steel can be used } \\
\text { for the apparatus; } \\
\text { No coloring impurities such as biphenyl } \\
\text { ketone compounds are present so good color } \\
\text { tone is easily achieved; } \\
\text { Product purity } ~ 99 \% \text {. }\end{array}$ & $14,34,35$ \\
\hline
\end{tabular}




\subsection{Technical Aspects of TPA Process}

\subsubsection{Main chemical reactions}

The commercial MC process involves a TPA synthesis step by the oxidation of $p \mathrm{X}$ and a TPA purification step by the hydrogenation of 4-CBA. The overall stoichiometric equation for $p \mathrm{X}$ oxidation and 4-CBA hydrogenation is shown in eqns. 1.1 and 1.2 , respectively.

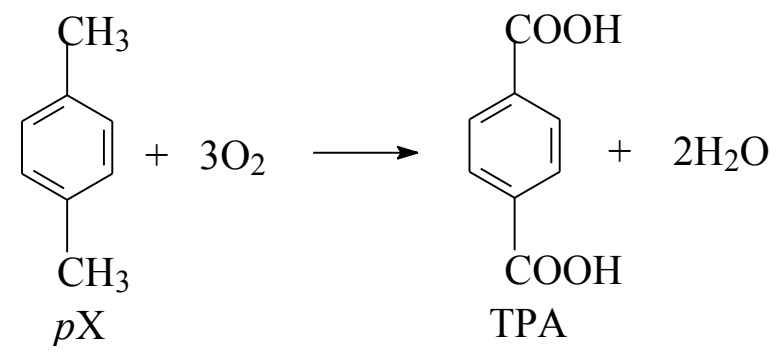

(eqn. 1.1)

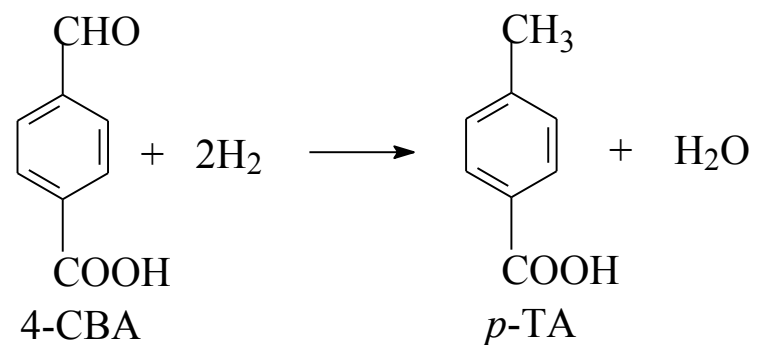

(eqn. 1.2)

\section{pXoxidation}

The $p \mathrm{X}$ oxidation is typically conducted at $190-205^{\circ} \mathrm{C}$ and $15-30$ bar [13]. A catalyst mixture of cobalt and manganese acetates is employed and hydrobromic acid or sodium bromide acts as the catalyst promoter reducing the induction period $[14,36]$. Aqueous acetic acid is used as the reaction medium. The oxidation reaction is highly exothermic with the heat of reaction of approximately $1,300 \mathrm{~kJ} / \mathrm{mol}$ for $p \mathrm{X}$ conversion to TPA $[37,38]$. The reaction involves a complex free radical chain mechanism [36] and is believed to proceed through the sequential reactions shown in Figure 1.3. 


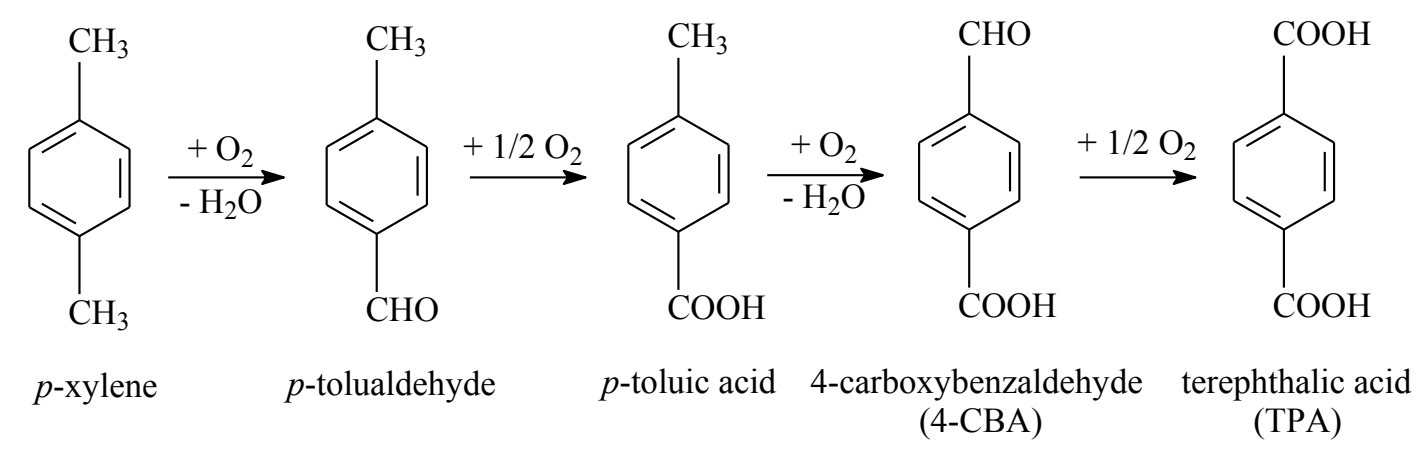

Figure 1.3: Sequential reaction network for $p \mathrm{X}$ oxidation to TPA

In addition to the intermediates shown in Figure 1.3, there are many other byproducts formed in the $p \mathrm{X}$ oxidation process to TPA. These impurities can be grouped into the derivatives of benzoic acid, phenol, terephthalic acid, diphenyl, fluorenone, benzophenone, anthraquinone, aromatic esters and bromine-substituted intermediates [39]. The formation of these compounds is due to the side reactions, radical and/or ionic, occurring among $p \mathrm{X}$, the oxidation intermediates, reaction solvent, and the catalytic system. The major impurities are 4-CBA, $p$-TA, $p$ tolualdehyde (TALD), benzoic acid, 1,4-benzenedimethanol diacetate and 4-hydroxymethyl benzoic acid (4-HMBA), the structures of which are shown in Figure 1.4. These byproducts are mostly present in the mother liquor and can be easily separated from the solid TPA product by crystallization under specified conditions. Some troublesome impurities such as 4-CBA tend to co-precipitate during the reaction and be embodied in the solid product obtained from the final step of the mother liquor separation. Hence, a subsequent purification step is needed to reduce these impurities to acceptable levels. 
$\overbrace{\mathrm{COOH}}^{\mathrm{CHO}}$

4-CBA

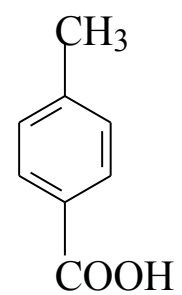

$p$-TA<smiles>Cc1ccc(C=O)cc1</smiles><smiles>O=C(O)c1ccccc1</smiles>

TALD benzoic acid

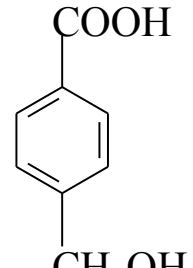<smiles>CC(=O)Oc1ccc(C(C)=O)cc1</smiles>

4-HMBA 1,4-benzenedimethanol diacetate

Figure 1.4: Structures of major impurities formed during $p \mathrm{X}$ oxidation to TPA

Over-oxidation of hydrocarbon reactant, intermediates and solvent (also termed as "burning" [40]) along with decarboxylation and decarbonylation form the undesired products such as $\mathrm{CO}$, $\mathrm{CO}_{2}$, water, benzoic acid, methyl acetate and methyl bromide. The formation of $\mathrm{CO}_{\mathrm{x}}(\mathrm{CO}$ and $\mathrm{CO}_{2}$ ) provides a measure of burning reactions and the $\mathrm{CO}_{2} / \mathrm{CO}$ molar ratio (the so-called "burning index") is typically around 3 [3] in the TPA process. It is estimated that $70 \%$ of acetic acid produced worldwide is used to manufacture TPA. Approximately $5 \%$ of the acetic acid is burned per pass [3]. This is equivalent to approximately $0.07 \mathrm{lb}$ of acetic acid burned per pound of TPA produced [6]. Another important byproduct during TPA production is methyl acetate, which is formed by the oxidation of acetic acid solvent (eqn. 1.3). Roffia et al. [41] studied the formation of methyl acetate and found that methyl acetate can be conveniently hydrolyzed over ion exchange catalysts, for example, to convert methyl acetate to methanol and acetic acid, which is then recycled.

$$
2 \mathrm{CH}_{3} \mathrm{COOH}+1 / 2 \mathrm{O}_{2} \rightarrow \mathrm{CH}_{3} \mathrm{COOCH}_{3}+\mathrm{H}_{2} \mathrm{O}+\mathrm{CO}_{2}
$$

\section{4-CBA hydrogenation}

The 4-CBA is dissolved with the crude TPA in water and then selectively converted to $p$-TA. The TPA re-dissolved in water is recrystallized from aqueous solution. The hydrogenation of 4CBA is typically carried out in a fixed-bed reactor containing a carbon-supported palladium 
catalyst to quantitatively convert 4-CBA to $p$-TA at $275-290{ }^{\circ} \mathrm{C}$ and $70-90$ bar $[8,10]$. The $p$-TA produced is then removed from the recrystallized TPA product by solid-liquid separation. The hydrogenation step also removes coloring in the TPA product by converting the yellow-colored fluorenones to the colorless fluorenes. The examples of structures of fluorenones and fluorenes are shown in Figure 1.5.

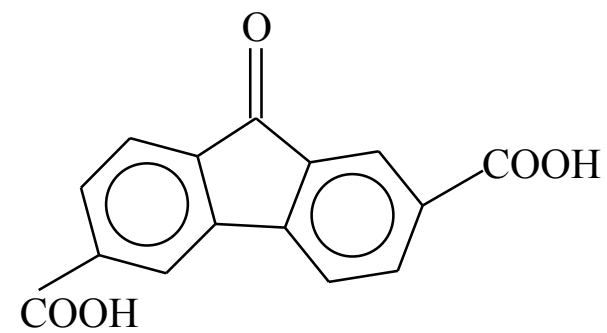

fluorenone

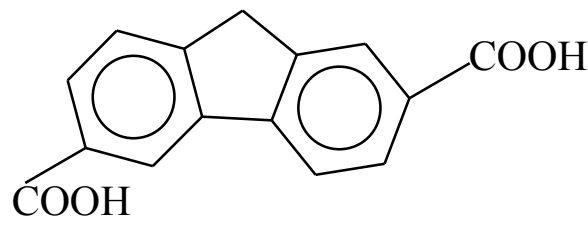

fluorene

Figure 1.5: Structures of fluorenones and fluorenes

\subsubsection{Product quality specifications}

The TPA product that is used in the polyester industry must satisfy stringent product specifications. Impurities with only one carboxylic acid group, such as 4-CBA, $p$-TA and benzoic acid, are polymerization terminators in the polymerization process for PET production and must be limited to very low levels. Trifunctional carboxylic acids can cause chain branching resulting in undesirable rheological and spinning properties. Inorganic impurities such as residual Co and Mn contents must also be restricted to $<10 \mathrm{ppm}$. Color and particle size are also important specification parameters. Colored impurities such as 4-CBA and fluorenones can be incorporated into the polyester. In particular, it is believed that 4-CBA levels of 1000-4000 ppm in the crude TPA have a major impact on the polyester color. However, 4-CBA levels below 300 ppm are less of color concern. Particle size determines the flow and the handling of the crystal TPA product as well as the viscosity of the slurry when mixed with ethylene glycol. Table 1.2 
lists the quantitative TPA product specifications on impurity levels, color and particle size [42]. The acid number is typically determined by titration. A perfectly pure TPA sample will have an acid number of $675.5 \mathrm{mg} \mathrm{KOH} / \mathrm{g}$, but the extremely low impurity levels make the acid number meaningless as a quantitative indication of purity, and it is being phased out [42].

Table 1.2: TPA product specifications

\begin{tabular}{lccc}
\hline \multirow{2}{*}{ Item } & \multirow{2}{*}{ Specification } & \multicolumn{2}{c}{ Typical value } \\
\cline { 3 - 4 } & & PTA & MTA \\
\hline Acid number, mg KOH/g & $675 \pm 2$ & $673-675$ & $673-675$ \\
Organic impurities, ppm & & & \\
$\quad$ 4-CBA & $\leq 25$ & 15 & $250-500$ \\
$\quad p$-TA & $\leq 125 \pm 45$ & $100-250$ & $<50$ \\
Benzoic acid & - & $<5$ & $15-40$ \\
Acetic acid & - & Trace & $400-800$ \\
Trimellitic acid & - & $10-20$ & $40-100$ \\
Inorganic impurities & $\leq 9$ & & \\
$\quad{ }^{a}$ Metals, ppm & $\leq 15$ & $<2$ & $<2$ \\
$\quad{ }^{\mathrm{b}}$ Ash, ppm & $\leq 0.2$ & 0.1 & $<3$ \\
$\quad$ Water, wt $\%$ & - & $98.7-99.1$ & $<0.1$ \\
${ }^{\mathrm{c}}$ Color-L, $\%$ & - & $0.7-1.7$ & $97.5-99$ \\
Color-b & - & $100-150$ & $2-3$ \\
Particle size, $\mu \mathrm{m}$ & & & $65-85$ \\
\hline
\end{tabular}

${ }^{\mathrm{a}} \mathrm{Co}+\mathrm{Mn}+\mathrm{Fe}+\mathrm{Cr}+\mathrm{Ni}+\mathrm{Mo}+\mathrm{Ti}$.

${ }^{\mathrm{b}}$ Trace metal oxides.

${ }^{c}$ The color of TPA crystals is measured in terms of a tristimulus color scale such as the CIE $L^{*} a * b$ scale [43]. The $L^{*}$ value measures dark to light and ranges from 0 to 100 . The $b *$ value measures yellow to blue, with higher values indicating higher yellow intensity.

\subsubsection{Mechanism of $p \mathrm{X}$ oxidation with MC catalyst}

The liquid-phase catalytic oxidation of $p \mathrm{X}$ with the $\mathrm{MC}$ catalytic system $(\mathrm{Co} / \mathrm{Mn} / \mathrm{Br})$ is generally believed to follow a free radical chain mechanism [36]. As other free radical chain reactions generally have, the oxidation reaction of $p \mathrm{X}$ to TPA involves initiation, propagation 
and termination steps. The model for redox activity of the MC catalyst [6] and the three steps of free radical chain reactions [44] are shown in Figures 1.6 and 1.7, respectively. In Figure 1.7, M denotes metal catalyst such as Co or Mn, R denotes aromatic reactants and I denotes radicals produced.

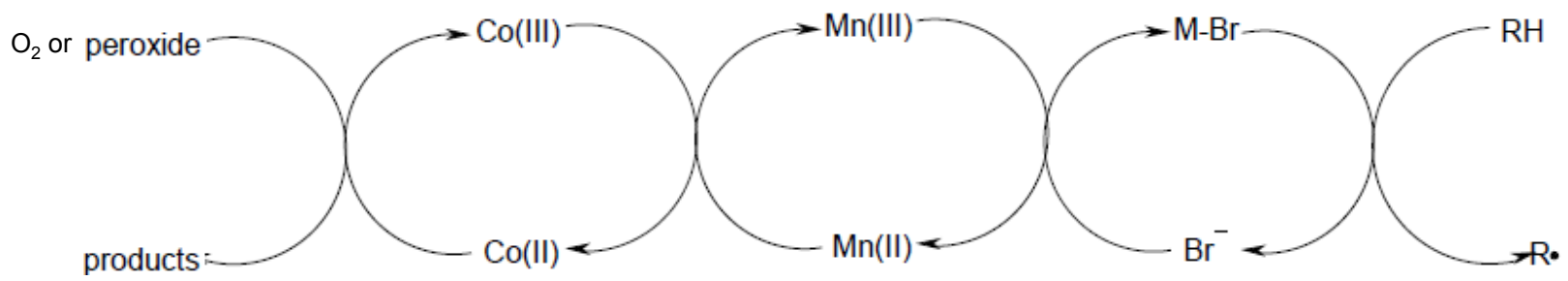

Figure 1.6: Model for redox activity of MC catalyst components [6]

$$
\begin{aligned}
& \mathrm{M}^{3+}+\mathrm{Br}^{-} \rightarrow \mathrm{M}^{2+}+\mathrm{Br}^{*} \\
& \mathrm{Br}^{*}+\mathrm{RCH}_{3} \rightarrow \mathrm{RCH}_{2}^{\cdot}+\mathrm{H}^{+}+\mathrm{Br}^{-} \\
& \mathrm{RCH}_{2}+\mathrm{O}_{2} \rightarrow \mathrm{RCH}_{2} \mathrm{O}_{2} \cdot \\
& \mathrm{RCH}_{2} \mathrm{O}_{2}{ }^{\cdot}+\mathrm{M}^{2+} \rightarrow \mathrm{RCHO}+\mathrm{M}^{3+}+\mathrm{OH}^{-} \\
& \mathrm{RCHO}+\mathrm{M}^{3+} \rightarrow \mathrm{RCO}^{\bullet}+\mathrm{M}^{2+}+\mathrm{H}^{+} \\
& \mathrm{RCHO}+\mathrm{Br}^{*} \rightarrow \mathrm{RCO}^{*}+\mathrm{Br}^{-}+\mathrm{H}^{+} \\
& \mathrm{RCO}^{*}+\mathrm{O}_{2} \rightarrow \mathrm{RCO}_{3}{ }^{\circ} \\
& \mathrm{RCO}_{3}{ }^{*}+\mathrm{RH} \rightarrow \mathrm{RCO}_{3} \mathrm{H}+\mathrm{R}^{*} \\
& \mathrm{RCO}_{3} \mathrm{H}+\mathrm{RCHO} \rightarrow 2 \mathrm{RCOOH} \\
& \mathrm{RCO}_{3} \mathrm{H}+\mathrm{M}^{2+} \rightarrow \mathrm{RCO}_{2}^{\cdot}+\mathrm{M}^{3+}+\mathrm{OH}^{-} \\
& \mathrm{RCO}_{2}{ }^{\circ}+\mathrm{RH} \rightarrow \mathrm{RCOOH}+\mathrm{R}^{*} \\
& \mathrm{I}_{i}^{*}+\mathrm{I}_{i}^{*} \rightarrow \mathrm{I}_{i} \mathrm{I}_{j}
\end{aligned}
$$

Figure 1.7: Initiation, propagation and termination steps of free radical chain reactions during $p \mathrm{X}$ oxidation to TPA with $\mathrm{Co} / \mathrm{Mn} / \mathrm{Br}$ system [44] 
The initiation step involves the formation of a highly unstable radical of $p \mathrm{X}$ by hydrogen atom abstraction. Even though Co(III) is capable of performing hydrogen abstraction to make the radical of $p \mathrm{X}$, using cobalt as the sole catalyst is inefficient because the hydrogen abstraction is very slow. It is well accepted that the initiation mechanism is predominantly due to bromine radicals originated from bromine ions that are oxidized by $\mathrm{Co}(\mathrm{III})$ and $\mathrm{Mn}(\mathrm{III})$. The formation of the radical of $p \mathrm{X}$ invokes the classical chain reaction scheme and greatly amplifies the initiation sequence via a propagation step. The propagation steps consist of oxidation of the substrates by molecular oxygen, metal ion, and bromine radical, and the reduction of radicals and hydroperoxides by metal ion. A substance of interest is the peroxy acid that is formed from oxygenated radicals like benzaldehydes, which is the basis of the Eastman-Kodak process and the Toray process (refer to Table 1.1), in which aldehydes are added as promoters due to their ability to form the peroxy acid as the radical initiating specie. In addition, owing to the electronwithdrawing nature of the carboxyl group, the oxidation of the second methyl group (i.e., the methyl group on the $p-\mathrm{TA}$ ) is more challenging. Heiba et al. [45] found that the rate of reaction of Co(III) in acetic acid of $p$-TA is 26 times slower than that of $p \mathrm{X}$. The addition of acetaldehyde in the Eastman-Kodak process and the Toray process can effectively resolve the problem of the termination of oxygenation caused by the electronic deactivation of the ring. The termination step consists of the reactions between two radicals to form the TPA product and the byproducts.

Partenheimer [46] illustrated the functions of the $\mathrm{Co} / \mathrm{Mn} / \mathrm{Br}$ catalyst in the oxidation of $p \mathrm{X}$ to TPA. Generally, according to his chemical model, cobalt appears unique among the first row transition metals in its weak reaction with acetic acid, which produces a small amount of $\mathrm{Co}(\mathrm{III})$ to initiate the reaction. When bromide is added, there is a large increase in activity and selectivity due to the rapid electron transfer from cobalt to bromide. In addition, there is a competition for 
Co(III) by methylbenzene (to make the radical of $p$-TA) and decarboxylation of acetic acid. At high temperatures, most of the Co(III) is being wasted in the decarboxylation of the solvent. The addition of bromide raises the temperature barrier to increase the reaction rate of the oxidation of the second methyl group. Manganese operates in much the same way as bromide by rapid electron transfer between cobalt and manganese species. The synergy effect between manganese and cobalt further increases the activity and selectivity as well as decreases decarboxylation reactions. In addition, given that manganese costs less than cobalt there is an economic incentive to reduce the cobalt usage.

\subsubsection{Kinetics of $p \mathrm{X}$ oxidation to TPA}

The intrinsic kinetic rates of the sequential steps during $p \mathrm{X}$ oxidation under MC process conditions $\left(200{ }^{\circ} \mathrm{C}, 15\right.$ bar) are extremely fast and tend to be limited by gas-liquid mass transfer resistance. Due to this reason, obtaining reliable kinetics of $p \mathrm{X}$ oxidation to TPA is a challenge. Some reported kinetic studies are under low temperatures or under conditions that are far away from the industrial reaction conditions to slow down the reactions. To the best of our knowledge, all the reported kinetic studies of $p \mathrm{X}$ oxidation to TPA are based on relatively high $p \mathrm{X}$ concentrations at which the TPA product will exceed its solubility and precipitate resulting in a gas-liquid-solid reaction system. Literature review of kinetic study of liquid phase $p \mathrm{X}$ oxidation to TPA will be summarized in section 2.2 of Chapter 2 .

\subsubsection{Heat management}

The reaction of $p \mathrm{X}$ oxidation is highly exothermic and the heat of reaction can reach approximately $1,300 \mathrm{~kJ} / \mathrm{mol}$ at the total conversion of $p \mathrm{X}$ to TPA [37, 38]. Heat removal with the use of jacket cooling or cooling coils in the reactor or an external heat exchanger could be ineffective because fouling is important in this case due to the precipitation of TPA. Here 
evaporative cooling is a preferable heat removal method compared to the others. Evaporative cooling reactors, also known as autorefrigerated reactors, are chemical reactors in which the cooling of the liquid phase where the reaction takes place is achieved by partial evaporation of the liquid [47]. The heat generation due to the oxidation of $p \mathrm{X}$ to TPA in industrial oxidation reactors is partly controlled by the evaporative cooling of the solvent (acetic acid) [48]. The latent heat of evaporation of acetic acid plays a significant role in thermal management as large quantities of the solvent are used in this process. A mathematical model of an evaporative cooling reactor operated at typical MC process conditions will be developed to quantify the effect of the solvent evaporation as a main source of reactor cooling in section 2.1.2.

\subsubsection{Technical developments}

Incremental technical developments have been made during the last several decades to improve the TPA process. Efforts towards developing greener TPA technologies have focused on several fronts such as the elimination of the hydrogenation step (the aforementioned MTA process is a typical example), an alternative solvent that is less susceptible than acetic acid to burning, milder reaction conditions and less corrosive catalytic system that avoids the use of bromine. This section reviews the developments of significance in this area.

\subsubsection{Reaction conditions}

\section{Acetic acid to $p X$ ratio in the feed}

The typical acetic acid solvent to $p \mathrm{X}$ weight ratio in the reactor feed is 2-4 in the MC process. Mitsui Petrochemical [49] reported a TPA process wherein this ratio falls in the range of 6.5-15, leading to a solid TPA concentration in the oxidation reactor below $20 \mathrm{wt} \%$. The residence time was reported to be in the range of 4.5-45 minutes. This set of operating conditions is claimed to 
facilitate the diffusion of oxygen into the reaction medium and to allow operation at a shorter residence time than that in the conventional $\mathrm{MC}$ process resulting in increased volumetric productivity in the reactor and an efficient production of aromatic carboxylic acids. However, the higher acetic acid throughput will require greater equipment sizing and higher energy input, resulting in an adverse economic and environmental impact. The effects of acetic acid to $p \mathrm{X}$ ratio in the feed on temperature control and on process economics and environmental impacts will be discussed in Chapters 2 and 4, respectively.

\section{$\underline{\mathrm{Co} / \mathrm{Mn} / \mathrm{Br} \text { ratio }}$}

As explained in section 1.3.3, cobalt and manganese exhibit synergy in the overall mechanism. Cheng et al. [50] reported that the variation of the $\mathrm{Co} / \mathrm{Mn}$ ratio had a strong effect on the activity and selectivity of the catalyst system. For the main reaction ( $p \mathrm{X} \rightarrow \mathrm{TPA})$, Cheng et al. reported an optimum $\mathrm{Co} / \mathrm{Mn}$ ratio at which the rates of the two intermediate oxidation steps of $p$-TA and 4-CBA (Figure 1.3) attained a maximum. The optimum $\mathrm{Co} / \mathrm{Mn}$ ratio decreased with an increase of reaction temperature. However, for the side reactions, the rate increased monotonically with the increase of $\mathrm{Co} / \mathrm{Mn}$ ratio. In addition, since cobalt is more expensive than manganese, more manganese is typically used than cobalt. The molar $\mathrm{Mn} / \mathrm{Co}$ ratio in the MC process is typically between 1 and 3 .

The role of bromide in the MC catalyst system has also been described in section 1.3.3. Kamiya et al. [51] investigated the effect of bromine/metals ratio on the reaction rate and found that at a cobalt concentration of $0.05 \mathrm{M}$, the $p \mathrm{X} \rightarrow$ TPA oxidation rate rapidly increased to a maximum value as the molar $\mathrm{Br} / \mathrm{Co}$ ratio was increased to 1 and remained constant up to a ratio of 9. The optimum $\mathrm{Br} / \mathrm{Co}$ ratio, at which the $p \mathrm{X} \rightarrow$ TPA oxidation rate attains a maximum, increased when lowering the cobalt concentration. However, considering the equipment 
corrosion issue and the production of methyl bromide, an ozone depleting gas, high levels of bromide should be avoided. The operating molar $\mathrm{Br} / \mathrm{Co}$ ratio is typically $0.5-1$ in the $\mathrm{MC}$ process [52]. On the other hand, too low a concentration of bromide can cause precipitation of the manganese resulting in $\mathrm{MnO}_{2}$, leading to a grey color in the solid TPA product. Incorporating one or more types of transition or lanthanide metal components as additives to the $\mathrm{MC}$ catalyst can effectively alleviate this problem. Further, most of these additives were reported to also increase catalytic activity [53-59].

Amoco Corporation [53] reported that the addition of hafnium $[<250$ ppmw (parts per million by weight) of the total reactor mother liquor] to the $\mathrm{Co} / \mathrm{Mn} / \mathrm{Br}$ catalyst system could increase the catalytic activity and allow a reduction in the net catalyst amounts required, leading to a reduction in bromine emissions. In one of their more recent patents [54], cerium was used as a catalyst additive and the molar ratio of bromine to total cobalt and manganese could be reduced to $<0.3$ by adding $0.1-0.3$ mole of cerium per mole of cobalt. The addition of molybdenum [55] has also been shown to enhance catalytic activity as manifested by higher yields of aromatic acids. In addition, zirconium [56-58] has also been proven to activate a $\mathrm{Co} / \mathrm{Mn} / \mathrm{Br}$ catalyst and to enhance the rate of oxidation of $p X$. Samsung General Chemicals [59] reported an alkali metal or alkaline earth metal (preferably $\mathrm{K}, \mathrm{Na}$, or $\mathrm{Cs}$ ) as additives to the $\mathrm{Co} / \mathrm{Mn} / \mathrm{Br}$ catalyst system and found that such additives enhanced both the yield as well as purity of the TPA product. Thus, in addition to alleviating Mn precipitation, the aforementioned catalyst additives are to some extent able to also improve the catalytic activity allowing the use of reduced amounts of catalytic components. The reduction in $\mathrm{Br}$ usage should significantly alleviate the environmental and equipment corrosion problems. 


\section{$\underline{\text { Co-feeds }}$}

Use of hydrocarbon additives as co-feeds with $p \mathrm{X}$ has been reported. Mitsubishi Chemical [60] incorporated 3-35 wt $\%$ of $p$-tolualdehyde in the $p \mathrm{X}$ feed and reported that the additive could reduce the acetic acid solvent burning rate and improve the solid TPA product quality. Mitsui Petrochemical [61] claimed that adding $0.5 \mathrm{ppm}$ to $1 \mathrm{wt} \%$ of $n$-eicosane to the $p \mathrm{X}$ feed prevented foaming of the reaction mixture and improved the volumetric production efficiency in the

oxidation reactor. Use of $\mathrm{CO}_{2}$ additive as co-feed was first reported by Samsung General Chemicals [62]. In their patent, a feed gas containing oxygen and at least 4 vol\% of $\mathrm{CO}_{2}$ was used to oxidize $p \mathrm{X}$ to TPA. It was reported that the addition of $\mathrm{CO}_{2}$ enhanced the TPA yield by nearly $8 \%$.

\section{Oxygen-enriched feed gas}

A number of patents $[48,63-66]$ deal with the use of pure or nearly pure oxygen or oxygenenriched air as the feed gas for $p \mathrm{X}$ oxidation. This method was aimed to reduce the compressor energy, the amount of vent gas treated and the operating pressure. However, the inventions do not address gas-phase flammability and associated safety issues. The use of oxygen-enriched gas has not yet been commercialized.

Zuo et al. [3] investigated the use of $\mathrm{CO}_{2}$-expanded liquids (CXLs) for $p \mathrm{X}$ oxidation to TPA and found that the reaction with a $\mathrm{CO}_{2} / \mathrm{O}_{2}$ mixture (with the maximum $\mathrm{O}_{2}$ content being $50 \mathrm{vol} \%$ ) instead of the traditional air oxidation system at lower temperatures (around $160{ }^{\circ} \mathrm{C}$ ) increases the yield and purity of the TPA product while significantly reducing solvent burning. Since $\mathrm{CO}_{2}$ has higher heat capacity and superior flame inhibition properties relative to nitrogen, oxygenenriched gas could be used as the reaction oxidant. The reduction in the flammability envelope in the pressure of $\mathrm{CO}_{2}$ will be addressed in more detail in Chapter 2. 


\subsubsection{Alternative catalyst}

Efforts have been studied over the years to seek alternative catalyst to the halide promoters in order to resolve the environmental and equipment corrosion problems. Shell Oil Company [67] reported that using a Co-Zr catalyst system between 80 and $130{ }^{\circ} \mathrm{C}$ and $2-3 \mathrm{~atm}$ in a stainless steel stirred tank reactor gave the $p \mathrm{X}$ conversion and TPA selectivity exceeding $90 \%$. Chester $e t$ al. [68] found that the optimum $\mathrm{Co} / \mathrm{Zr}$ ratio in neat acetic acid was 6-7 for maximum TPA yield and minimum induction period. Promotors such as $\mathrm{CoBr}_{2}-\mathrm{MnBr}_{2}[69,70]$ instead of $\mathrm{HBr}$ as a bromine source, $N$-hydroxyphthalimide [71,72], and $N$-hydroxy succinimide $[73,74]$ have also been reported for $p \mathrm{X}$ oxidation to TPA under milder conditions $\left(\sim 100^{\circ} \mathrm{C}\right)$, but the $p \mathrm{X}$ conversion and TPA selectivity are below the typical industrial values.

\subsubsection{Alternative solvent}

Another promising alternative is to carry out the reaction of $p \mathrm{X}$ oxidation to TPA in a solvent other than acetic acid. The use of either supercritical or high-temperature liquid water as a benign substitute for acetic acid has been reported [75-78]. The reaction systems were necessarily operated at high temperatures $\left(330-450{ }^{\circ} \mathrm{C}\right)$ and high pressures $(250-270$ bar) with TPA yields of $>90 \%$. The absence of acetic acid reduced the methyl bromide formation and $\mathrm{CO}_{2}$ emissions, and also eliminated the distillation column for acetic acid/water separation. A solvent comprising of benzoic acid and water has been reported by BP for the oxidation of $p \mathrm{X}$ to TPA [79]. Benzoic acid is easier to separate from water than acetic acid. Further, the use of benzoic acid allowed solid-liquid separation at elevated temperatures, leading to reduced co-crystallization of the product impurities. Dow chemical [80] reported an aqueous medium containing at least $30 \mathrm{wt} \%$ water and preferably up to $30 \mathrm{wt} \%$ surfactant such as stearic acid or $\alpha$-olefin sulfonate and a low molecular weight material containing a hydrophilic end group as co-surfactant such as acetic 
acid or benzoic acid for $p \mathrm{X}$ oxidation. To the best of our knowledge, none of these alternative solvents have been applied commercially yet.

The above literature review outlined only the major technical developments regarding the oxidation reactor. In addition, a great number of patent applications deal with reactor design, unit operations for crystallization, product drying, acetic acid solvent dehydration, off-gas treatment,

hydrogenation reactor, energy recovery method, and solid-liquid separation techniques. One should note that this literature review is by no means exhaustive as the volume of work reported on the TPA process is quite extensive.

\subsection{Challenges in the MC Process}

Although the MC process for TPA production has been practiced since the 1950s and has long been considered to be a mature technology, there are several challenges that remain to be addressed or improved. The major issues of the MC process are as follows.

\section{Hydrogenation step}

The polymer grade TPA product is achieved following complex purification steps involving hydrogenation under harsh reaction conditions (high temperature and pressure) using expensive metal catalysts as well as a number of additional solid-liquid separation steps. The TPA purification by hydrogenation doubles capital investment and increases operating costs by several cents/lb TPA [81]. Further, the fossil fuel-based hydrogen production and energy generation required for the hydrogenation step increases the environmental burdens.

\section{Solvent burning}

Roughly $5 \%$ of the acetic acid is destroyed by oxidative burning per pass, resulting in significant solvent loss and $\mathrm{CO}_{2}$ emissions. The burning of acetic acid is considered to be one of 
the primary contributors to the variable cost in the production of TPA [6]. Therefore, solvent burning is a significant factor in the economics of the MC process.

\section{Corrosion issues}

Due to the corroding capability of acetic acid and hydrogen bromide at high operating temperatures toward the conventional material of construction apparatus such as stainless steel, titanium-lining is required for the oxidation reactor and some of the columns and heat exchangers, resulting in higher capital costs. Besides, the use of titanium only alleviates the corrosion and has limitations of its own.

\section{Safety issues}

The use of a flammable organic solvent (acetic acid) during the $p \mathrm{X}$ oxidation to TPA gives rise to vapor phase flammability concerns. Air is used as the primary oxidant instead of pure $\mathrm{O}_{2}$ or oxygen-enriched air for safety reasons. The upper flammability limit (UFL) for acetic acid in air under ambient or elevated pressure (above 30 bar) is below 25 vol\% and the lower flammability limit (LFL) is approximately 3 vol\% $[6,82]$. The oxidation reaction should be operated outside the flammability envelope. In addition, there exists an inherent safety concern,

owning to titanium burning under oxygen-enriched atmospheres at high temperatures [6]. Both of these safety concerns necessitate monitoring and control of the oxygen content in the vapor phase.

\section{Off-Gas treatment}

Gaseous emissions from the TPA process are mostly the oxygen-depleted air from the oxidation step [83]. Volumes are large and the vent gas contaminated with VOCs (volatile organic compounds), $\mathrm{CO}_{\mathrm{x}}$ and $\mathrm{NO}_{\mathrm{x}}$ must be cleansed via catalytic combustion followed by 
scrubbing to meet the most demanding regulations for process vents. The large volumes of offgases requiring treatment increases process costs.

\subsection{Objectives and Organization of This Work}

This dissertation is aimed at developing a new process concept for TPA production that promotes process sustainability by solving some of the existing process challenges explained above. Toward this end, this dissertation introduces a novel spray reactor system for the oxidation of $p \mathrm{X}$ to TPA. The overall project goal is to investigate the feasibility and potential benefits of the spray reactor for TPA production - in terms of rational reactor design and safe operation, superior product yield and purity, economic viability and environmental sustainability

- employing complementary experimental and theoretical approaches for process development. Specific objectives are to:

(1) Perform kinetic study of $p \mathrm{X}$ liquid phase oxidation to TPA in a stirred reactor under homogeneous conditions (i.e., low $p \mathrm{X}$ concentrations employed to avoid TPA precipitation);

(2) Develop and demonstrate a laboratory-scale spray reactor system for $p \mathrm{X}$ oxidation to TPA, benchmarking the process with performance metrics ( $p \mathrm{X}$ conversion, TPA yield and purity, etc.) from a conventional stirred reaction configuration;

(3) Develop and demonstrate a laboratory-scale continuous spray process.

(4) Perform comparative economic and environmental impact assessments of the MC process and spray process for TPA production, based on plant-scale process simulations.

The various chapters following this introductory chapter are organized as follows. In the next chapter of this dissertation, the kinetic study of $p \mathrm{X}$ liquid phase oxidation to TPA is investigated in a stirred reactor under homogeneous conditions employing low $p \mathrm{X}$ concentrations at which 
product precipitation is avoided. A pseudo first-order kinetic model is proposed and kinetic rate constants are determined based on the experimental data. In Chapter 3, a semi-batch spray reactor is developed and demonstrated to produce high-purity TPA in one step. Theoretical analyses based on reactor engineering fundamentals such as $\mathrm{O}_{2}$ availability throughout the liquid phase, reactor stability and vapor phase flammability issues are considered in order to rationally design the reactor. Reaction parameters such as $p \mathrm{X}$ conversion, TPA yield and purity are examined to test the performance of the spray reactor. Chapter 4 describes the methodology and procedures to develop a continuous spray process. Some preliminary experimental results from the continuous spray reactor are reported. Chapter 5 covers the economic and environmental aspects of the conceptual spray process based on plant-scale simulation to assess the economic viability and the environmental benignity of the alternative spray process for TPA production. Finally, in Chapter 6, a summary of the conclusions of the current work and recommendations for future work are provided. 


\section{Chapter 2 Kinetic Studies of Homogeneous p-Xylene Oxidation to Terephthalic Acid in Acetic Acid with a Co/Mn/Br Catalyst}

In this chapter, the intrinsic kinetics of $p \mathrm{X}$ liquid phase oxidation to TPA is investigated in a stirred reactor under homogeneous conditions without product precipitation by employing low initial $p \mathrm{X}$ concentrations in order to avoid interphase gas-liquid and gas-solid mass transfer limitations. The kinetic study is important to the rational design and optimization of the CEBC spray process as well as to aid in reactor modeling studies. Before getting started, solubility studies are first performed to determine the homogeneous reaction conditions at which the TPA remains dissolved in solution.

\subsection{Solubility Studies of the MC Process Related Components}

\subsubsection{Motivation}

The intrinsic solubilities of TPA, 4-CBA, and $p$-TA are generally considered in industrial TPA purification by crystallization. TPA is purified from the mixture of acetic acid and water as follows. In the oxidation section, the crude TPA product is recovered by a series of flash crystallizers (3-6 stages) with stepwise cooling [20]. In the subsequent hydrogenation section, the crude TPA is re-dissolved in water at elevated temperature of $275-290{ }^{\circ} \mathrm{C}[8,10]$, then purified by hydrotreating and finally passed through a series of 3-6 stage crystallizers $[10,84]$ with stepwise cooling to recover the purified TPA product from aqueous solution. Measurements of solubilities of the aforementioned compounds in aqueous acetic acid are important for the 
rational choice of operating conditions and for rational equipment design for the separation and purification steps.

In the case of the spray reactor, reliable solubility data are important to determine the concentrations at which the TPA remains dissolved in solution at the reaction conditions. If the TPA and impurities precipitate, the solid particles could trap liquid reaction mixture and give rise to $\mathrm{O}_{2}$ diffusion limitations resulting in incomplete oxidation. Intrinsic solubility studies will help determine the maximum $p \mathrm{X}$ concentration that may be used to maintain TPA dissolved in the liquid phase. These results will guide the choice of operating conditions that avoid solids formation during the kinetic studies (discussed in the next section) and during continuous operation to minimize $\mathrm{O}_{2}$ diffusion limitations as well as the possibility of valve plugging during continuous operation.

The solubilities of TPA, 4-CBA and $p$-TA in acetic acid/water solvent mixtures have been investigated by several research groups [85-89]. Most of these studies followed the procedure of first experimentally measuring the solubility using one of the following techniques: (a) steady state method by sampling and analyzing the saturated solution, or (b) laser technique by observing the equilibrium temperature, and then correlating the experimental data to various phase equilibrium models. Table 2.1 outlines the temperature ranges of the reported solubility data. These literature data were obtained in relatively limited temperature ranges that do not provide adequate guidance for our experiments. Hence, it was decided to perform solubility studies covering a relatively wide temperature range. 
Table 2.1: Temperature ranges of available literature solubility data

\begin{tabular}{cccc}
\hline Solute & Solvent & T Range & Ref. \\
\hline TPA & $\mathrm{HOAc} / \mathrm{H}_{2} \mathrm{O}(\mathrm{HOAc}=0-100 \mathrm{wt} \%)$ & $150-240{ }^{\circ} \mathrm{C}$ & $85-89$ \\
$4-\mathrm{CBA}$ & $\mathrm{HOAc} / \mathrm{H}_{2} \mathrm{O}(\mathrm{HOAc}=84.2 \mathrm{wt} \%$ or $100 \mathrm{wt} \%)$ & $15-56{ }^{\circ} \mathrm{C}$ & 87 \\
$p$-TA & $\mathrm{HOAc} / \mathrm{H}_{2} \mathrm{O}(\mathrm{HOAc}=84.2 \mathrm{wt} \%$ or $100 \mathrm{wt} \%)$ & $18-76{ }^{\circ} \mathrm{C}$ & 87 \\
\hline
\end{tabular}

\subsubsection{Experimental method}

The solubility studies were performed in a $50 \mathrm{~mL}$ stirred vessel (Parr Instrument Company, Series 4560 Mini Bench Top Reactor) with view windows. The experimental method is similar to that reported by Chen and Ma [87], except that instead of using a laser technique, we observed the dissolving process visually, which is more common and simpler. The experimental procedure for a typical run is as follows: (a) A certain amount of TPA and $20 \mathrm{~g}$ of solvent (pure acetic acid or acetic acid/water solvent mixture with $95 \mathrm{wt} \%$ acetic acid) are loaded in the $50 \mathrm{~mL}$ vessel; (b) The vessel is pressurized with $\mathrm{N}_{2}$ to 15 bar; (c) The vessel is heated and the $\mathrm{T}$ at which dissolution and reprecipitation occurs is visually noted. Figure 2.1 shows the typical T \& P profiles during the solubility studies. The vessel was heated very slowly and the stirring was started and stopped alternatively until no solute was precipitated from the solution, at which point the temperature would be the solubility temperature. The apparent oscillations in temperature and pressure rise and fall are due to the stirrer being turned on and off during the experiments. 


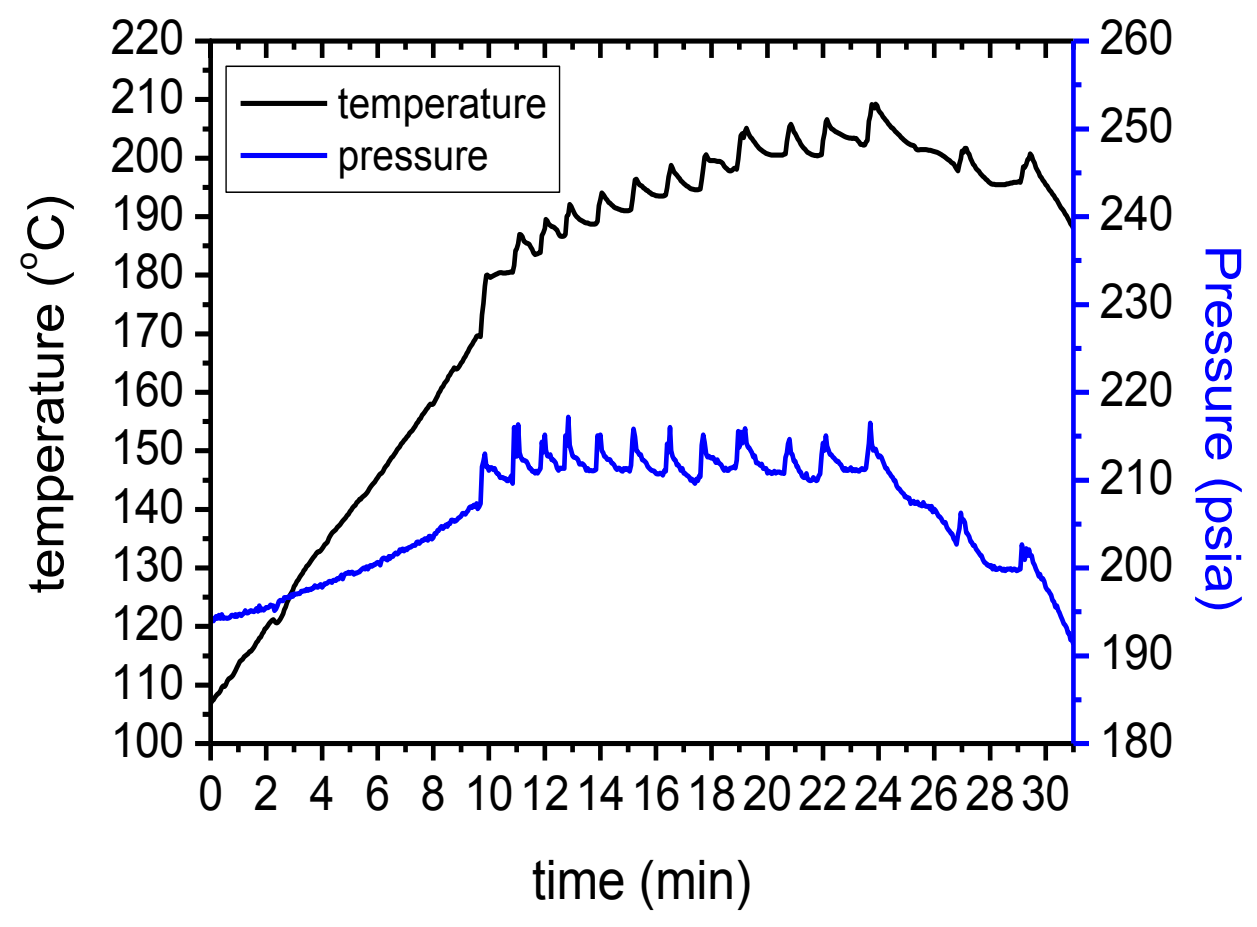

Figure 2.1: Typical T \& P profiles during solubility studies ( $\mathrm{T} \& \mathrm{P}$ rise and fall due to stirrer turned on and off deliberately)

\subsubsection{Experimental results}

Solubility data of TPA, 4-CBA and $p$-TA in a wide temperature range were obtained (Figure 2.2). Numerical data of the experimental solubilities are shown in Table A1 of Appendix A. The measured TPA solubility matches well with the literature data (Figure 2.2a) thus validating the experimental method. It was found that while water addition enhances TPA solubility in acetic acid at temperatures above $130{ }^{\circ} \mathrm{C}$, the solubility is unaffected below this temperature. The solubilities in acetic acid follow the order $p$-TA $>4$-CBA $>$ TPA, with each successive increase or decrease being approximately one order of magnitude. The reaction was carried out at $\mathrm{P}$ and $\mathrm{T}$ such that TPA remains soluble in the liquid phase at reactor conditions during kinetic studies and continuous runs. The TPA was precipitated post reactor by cooling. 
(a)

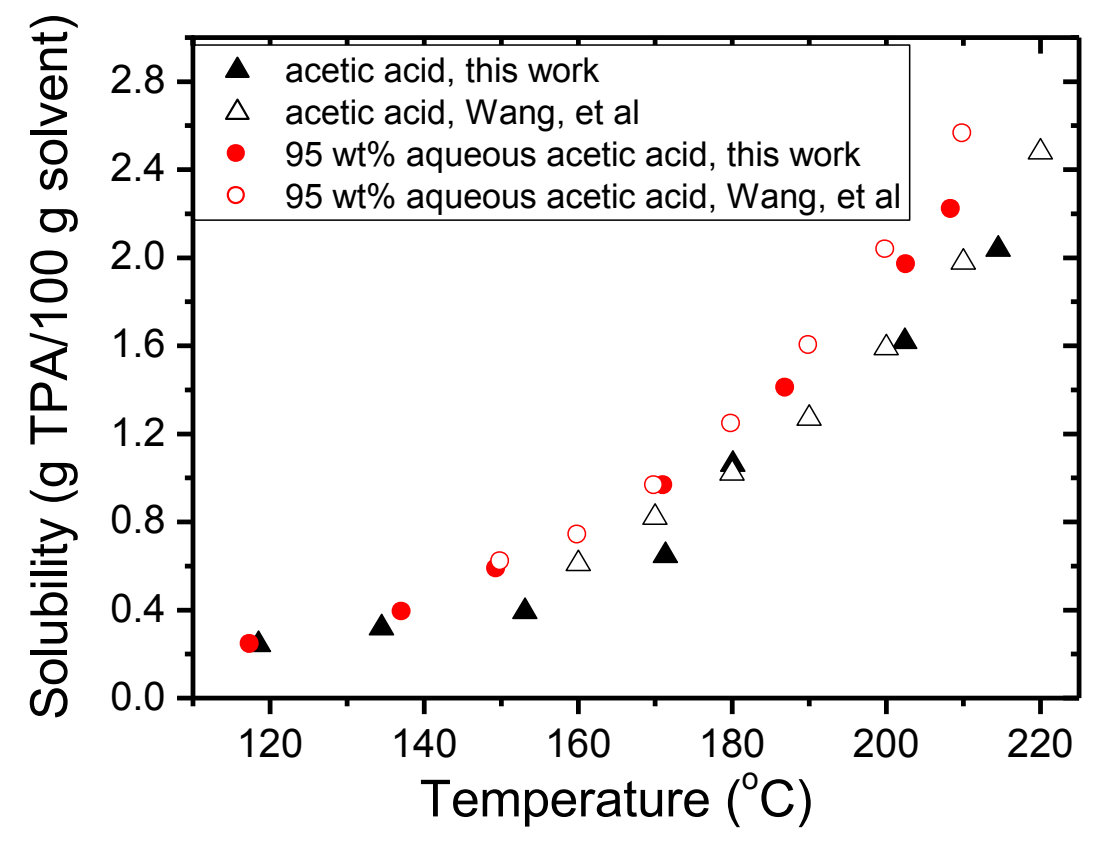

(b)

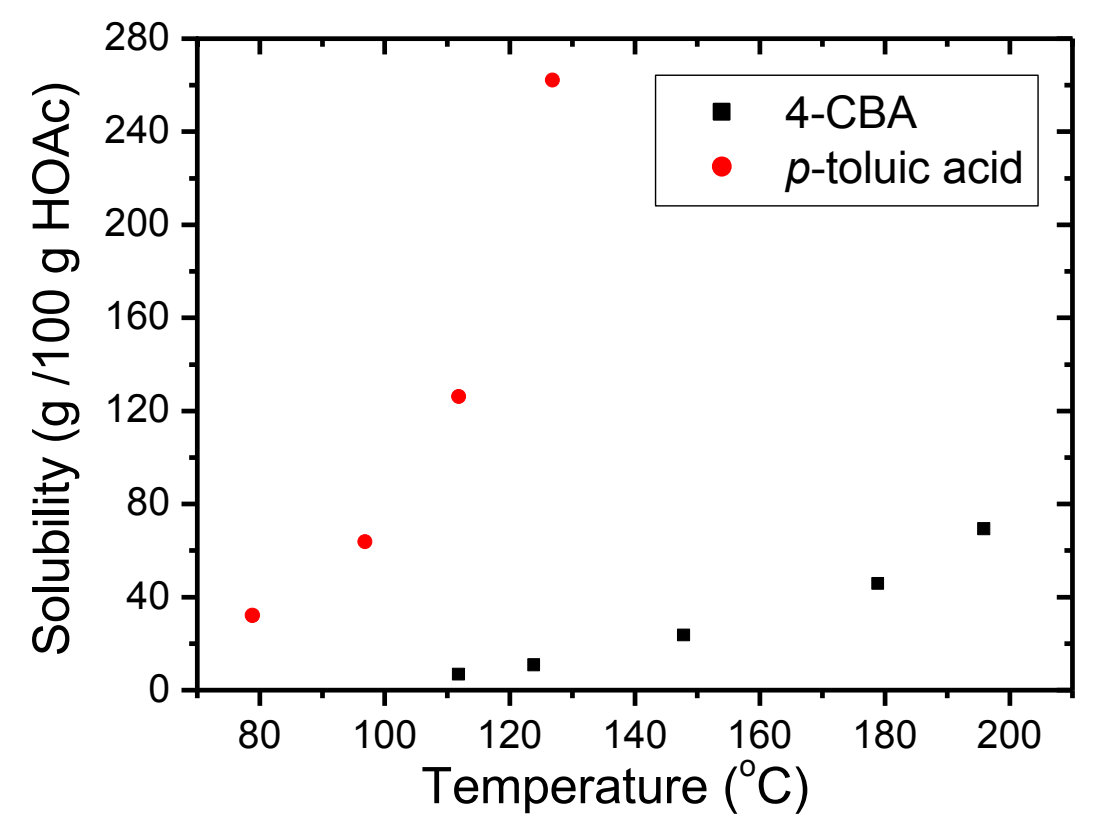

Figure 2.2: Solubilities of the MC process related components (14.5-15.5 bar in $\mathrm{N}_{2}$ ) (a) TPA; (b) 4-CBA and $p$-TA 


\subsection{Previous Work on Kinetics of $p X$ Oxidation to TPA}

Due to its industrial importance, the kinetics of liquid-phase $p \mathrm{X}$ oxidation to TPA has been the subject of several reports [90-99]. The catalytic oxidation of $p \mathrm{X}$ to TPA with $\mathrm{Co} / \mathrm{Mn} / \mathrm{Br}$ catalyst system is known to proceed via a free radical chain mechanism (refer to section 1.3.3 for details of the reaction mechanism) involving a large number of radical and molecular species. A detailed kinetic scheme including all the elementary reactions and intermediates has not been possible primarily due to the difficulties in detecting and monitoring the highly reactive radicals over time. Because of this, "lumped" kinetic schemes and models [93, 97-99] have been adopted to simplify this complex reaction by taking into account only the most important molecular intermediates and relatively stable intermediate products such as $p$-tolualdehyde (TALD), $p$ toluic acid ( $p$-TA), 4-carboxybenzaldehyde (4-CBA) and TPA.

The reported kinetic models of $p \mathrm{X}$ oxidation to TPA were developed based on the power law assumption or derived from the simplified radical chain reaction mechanisms. Cao and coworkers [94-97] investigated the kinetics of $p \mathrm{X}$ oxidation at low temperatures $\left(80-130{ }^{\circ} \mathrm{C}\right)$ and low pressure (1 atm) using methyl benzoate as the solvent and cobalt naphthenate and TALD as the catalyst and promoter, respectively. The rate of each oxidation step was assumed to be zero order in oxygen (when the oxygen partial pressure was greater than 50-100 Torr) and first-order with respect to the reactants being oxidized. A semi-batch reactor model which accounts for inter- and intra-phase gas-liquid mass transfer processes was presented and the agreement between model predictions and experimental data was shown to be satisfactory. Wang and coworkers $[44,98]$ developed a fractional kinetic model based on the complicated radical chain reaction mechanism and determined that the apparent reaction order was $<<1$ based on $p \mathrm{X}$ oxidation to TPA experiments performed under industrial oxidation conditions. Model 
parameters were determined from both batch and semi-continuous experimental results. Yan and co-workers $[99,100]$ extended the power law model and the fractional model to correlate the kinetic parameters from the industrial CSTR with those from the laboratory reactor using data mining approach based on artificial neural network (ANN). Researchers from the same institute as Yan $[93,101]$ developed a simplified free-radical reaction kinetic model in which peroxy radicals were involved in the lumped reaction equations. Table 2.2 lists the various kinetic models along with lumped reaction equations and reaction conditions from the available literature. 

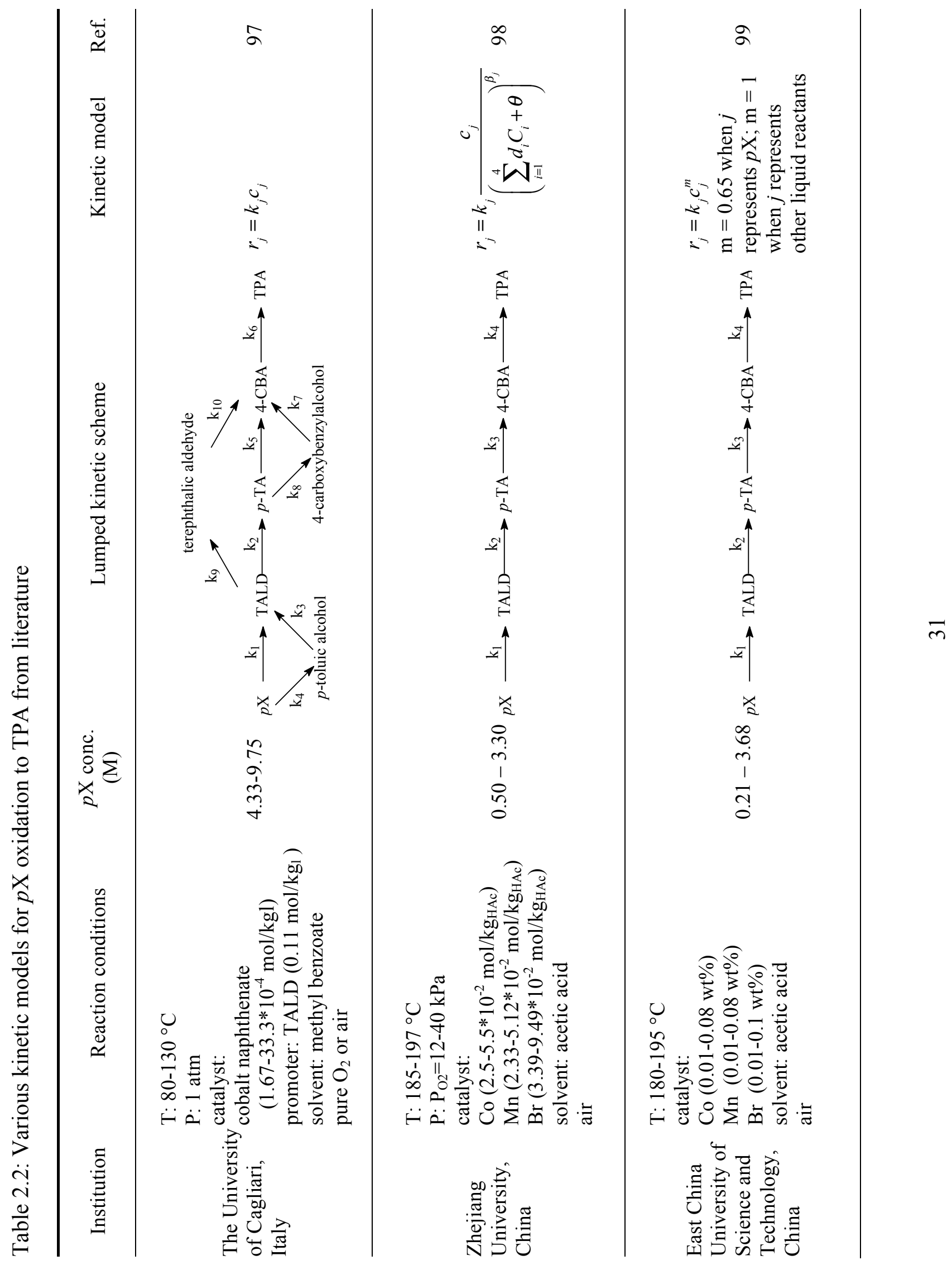


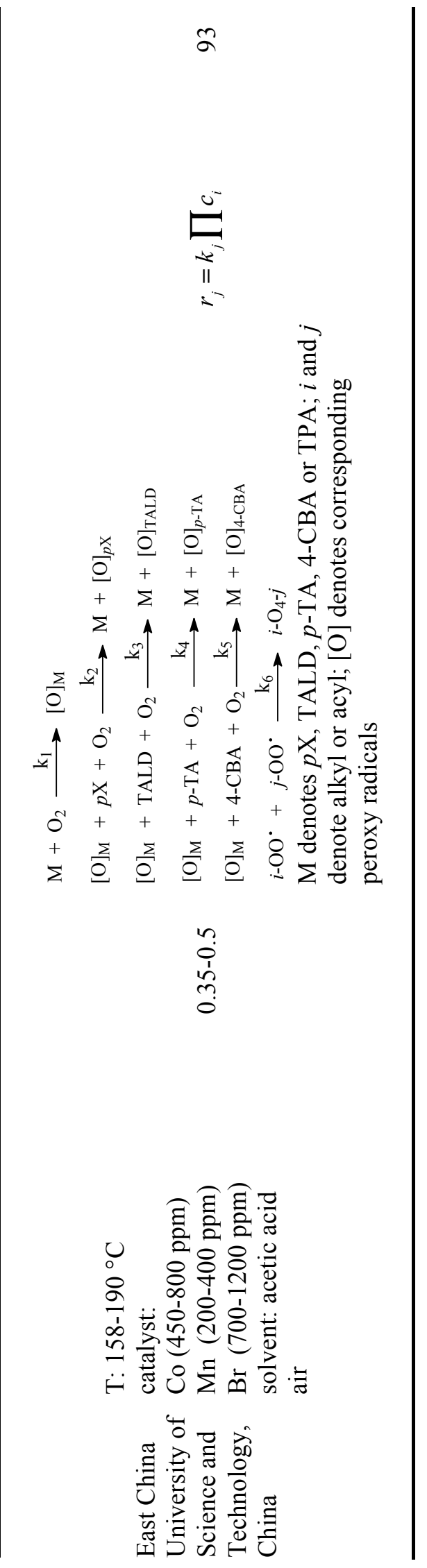

N 


\subsection{Motivation and Significance of This Work}

To the best of our knowledge, all the reported kinetic studies of $p \mathrm{X}$ oxidation to TPA are based on relatively high $p \mathrm{X}$ concentrations (Table 2.2) at which the TPA product will exceed its solubility and precipitate resulting in a gas-liquid-solid reaction system. The kinetic parameters obtained under these reaction conditions could be subject to interphase mass transfer limitations. If TPA precipitates as a solid, it traps some of the reaction intermediates into its solid structure removing the intermediates from solution. The trapped intermediates would then react slower. By eliminating the formation of solids in the liquid phase (lower $p \mathrm{X}$ concentrations are used to prevent TPA precipitation) gas-solid and liquid-solid mass transfer steps can be eliminated. Further, the various dissolved products can be more accurately sampled and analyzed.

In this work, the intrinsic kinetics of $p \mathrm{X}$ liquid phase oxidation to TPA was investigated in a stirred reactor under homogeneous conditions employing low $p \mathrm{X}$ concentrations at which product precipitation is avoided even at total $p \mathrm{X}$ conversion to TPA. In this manner, interphase gas-liquid and gas-solid mass transfer resistances that are prevalent in most reported kinetic studies are avoided. A first order kinetic model was assumed based on a lumped reaction scheme in which the oxygen concentration is maintained constant in stoichiometric excess for complete $p \mathrm{X}$ oxidation to TPA. Kinetic rate constants were obtained by non-linear regression of the experimental data to fit the model. Such a kinetic model is essential for the rational design, optimization and scale-up of the spray reactor. 


\subsection{Experimental}

\subsubsection{Materials}

The following chemicals were used as obtained from Sigma-Aldrich without further purification: $p \mathrm{X}$ (substrate), cobalt(II) acetate tetrahydrate (catalyst), manganese(II) acetate tetrahydrate (catalyst), hydrobromic acid (48\% in water) (promoter), acetic acid (solvent), biphenyl (internal standard). Ultra high purity (UHP) grade oxygen and industrial grade $\mathrm{CO}_{2}$ were purchased from Airgas and Linweld, respectively.

\subsubsection{Experimental setup and procedure}

The experimental apparatus employed in this work is shown in Figure 2.3. The $50 \mathrm{~mL}$ titanium stirred vessel (Parr Instrument Company, Series 4560 Mini Bench Top Reactor) was equipped with a magnetically driven impeller for stirring, a dual probe J-type thermocouple and an OMEGA transducer for measuring the reactor temperature and pressure. The temperature and pressure were continuously recorded and controlled with the help of a LabVIEW ${ }^{\circledR}$ data acquisition system. The reactor was modified to accommodate multiple inlet/outlet ports for (a) introducing $\mathrm{O}_{2}$ via a dip tube/sparger, (b) sampling the liquid phase via a 1/16" titanium dip tube, (c) withdrawing the vapor phase, and (d) providing a pressure relief valve. 


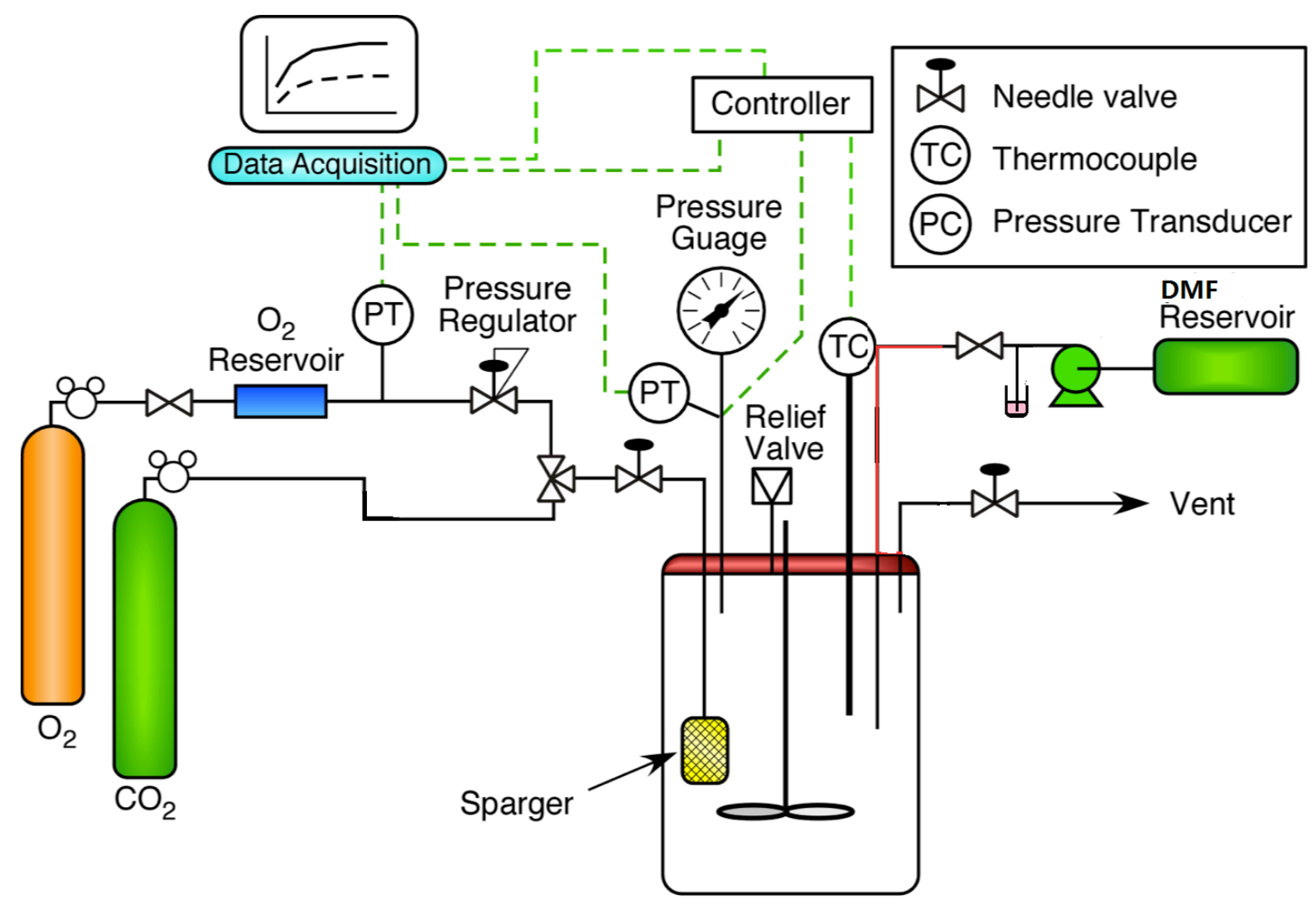

Figure 2.3: Experimental apparatus for $p \mathrm{X}$ oxidation to TPA

In a typical experimental run, the reactor was initially charged with a $35 \mathrm{~mL}$ solution of $p \mathrm{X}$, $\mathrm{Co} / \mathrm{Mn} / \mathrm{Br}$ catalyst and biphenyl in acetic acid. This homogeneous mixture is pressurized with $\mathrm{CO}_{2}$ to $2.5 \mathrm{bar}$, and then heated to the desired temperature. After the temperature and pressure stabilized, $\mathrm{O}_{2}$ was added to the reactor to initiate the oxidation reaction. The $\mathrm{O}_{2}$ consumed in the liquid phase by reaction was continuously replenished from an external reservoir such that $\mathrm{O}_{2}$ partial pressure (and therefore reactor pressure) was maintained constant. To avoid sampling errors due to product flashing and the TPA sticking to the inner wall of the tubing, the liquid phase was directly sampled into $15 \mathrm{~mL}$ DMF ( $N, N$-dimethylformamide) solvent. The titanium sampling line was heated to $200{ }^{\circ} \mathrm{C}$ to avoid TPA precipitation. The unheated line was flushed by DMF after each sampling to remove residual reactants and products from the line. Table 2.3 
summarizes the operating conditions of the $p \mathrm{X}$ oxidation experiments. Typical industrial conditions and operating conditions used for the reported kinetic study are also listed for comparison.

Table 2.3: Operating conditions of the $p \mathrm{X}$ oxidation experiments

\begin{tabular}{cccccccc}
\hline Run \# & Stirring rate & $\mathrm{T}\left({ }^{\circ} \mathrm{C}\right)$ & ${ }^{\mathrm{a}} \mathrm{P}_{\mathrm{O} 2}(\mathrm{bar})$ & $\mathrm{C}_{p \mathrm{X}, 0}(\mathrm{M})$ & $\mathrm{C}_{\mathrm{Co}}(\mathrm{M})$ & $\mathrm{C}_{\mathrm{Mn}}(\mathrm{M})$ & $\mathrm{C}_{\mathrm{Br}}(\mathrm{M})$ \\
\hline 1 & 600 & 200 & 3.4 & 0.025 & 0.0125 & 0.0125 & 0.035 \\
2 & 800 & 200 & 3.4 & 0.025 & 0.0125 & 0.0125 & 0.035 \\
3 & 1000 & 200 & 3.4 & 0.025 & 0.0125 & 0.0125 & 0.035 \\
4 & 1200 & 200 & 3.4 & 0.025 & 0.0125 & 0.0125 & 0.035 \\
5 & 1400 & 200 & 3.4 & 0.025 & 0.0125 & 0.0125 & 0.035 \\
6 & 1000 & 200 & 3.4 & 0.025 & 0.0125 & 0.0125 & 0.016 \\
7 & 1000 & 200 & 3.4 & 0.025 & 0.0125 & 0.0125 & 0.009 \\
8 & 1000 & 200 & 3.4 & 0.025 & 0.0125 & 0.0125 & 0.005 \\
9 & 1000 & 200 & 3.4 & 0.025 & 0.0125 & 0.0125 & 0.002 \\
10 & 1000 & 200 & 3.4 & 0.025 & 0.0125 & 0.0125 & 0.001 \\
11 & 1000 & 200 & 3.4 & 0.035 & 0.0125 & 0.0125 & 0.016 \\
12 & 1000 & 200 & 3.4 & 0.014 & 0.0125 & 0.0125 & 0.016 \\
Industry [20] & - & 195 & 0.5 & 3.8 & 0.006 & 0.017 & 0.01 \\
Wang et al $[98]$ & 800 & 191 & 0.4 & 0.9 & 0.3 & 0.3 & 0.9 \\
\hline
\end{tabular}

${ }^{a}$ The total pressure is 15 bar.

\subsubsection{Analytical techniques}

The reactant $(p \mathrm{X})$, intermediates (TALD, $p$-TA, BPTA and 4-CBA) and desired product (TPA) were analyzed by HPLC using the gradient elution technique described by Viola and Cao [102]. Specifically, the mobile phase consisted of three eluents: aqueous phase $(0.1 \mathrm{wt} \%$ phosphoric acid) and an organic phase consisting of 7 parts acetonitrile and 2 parts methanol by volume. The gradient elution program was as follows: at $0 \mathrm{~min}, 100 \mathrm{vol} \%$ aqueous phase; from 0 to $5 \mathrm{~min}$, the eluent mixture was changed linearly with time to 95 vol\% aqueous phase and 5 vol $\%$ organic phase; from 5 to $10 \mathrm{~min}$, the mixture composition changed linearly with time to become $40 \mathrm{vol} \%$ 
aqueous phase and $60 \mathrm{vol} \%$ organic phase; from 10 to $24 \mathrm{~min}$, the mixture composition was kept constant. The total flow rate of the mobile phase was $1 \mathrm{~mL} / \mathrm{min}$. A UV detector was used to quantify the products using biphenyl as internal standard (215 nm for $p \mathrm{X}$ and $254 \mathrm{~nm}$ for all other products). The HPLC samples were prepared from those collected during the reaction and adding $15 \mathrm{~mL}$ acetic acid to each sample. Figure 2.4 shows a typical chromatogram obtained from HPLC analysis of a sample. The carbon balance estimated from only the concentrations of the aromatic compounds was found to be $>95 \%$. In this work, the combustion reactions and volatilization were neglected.

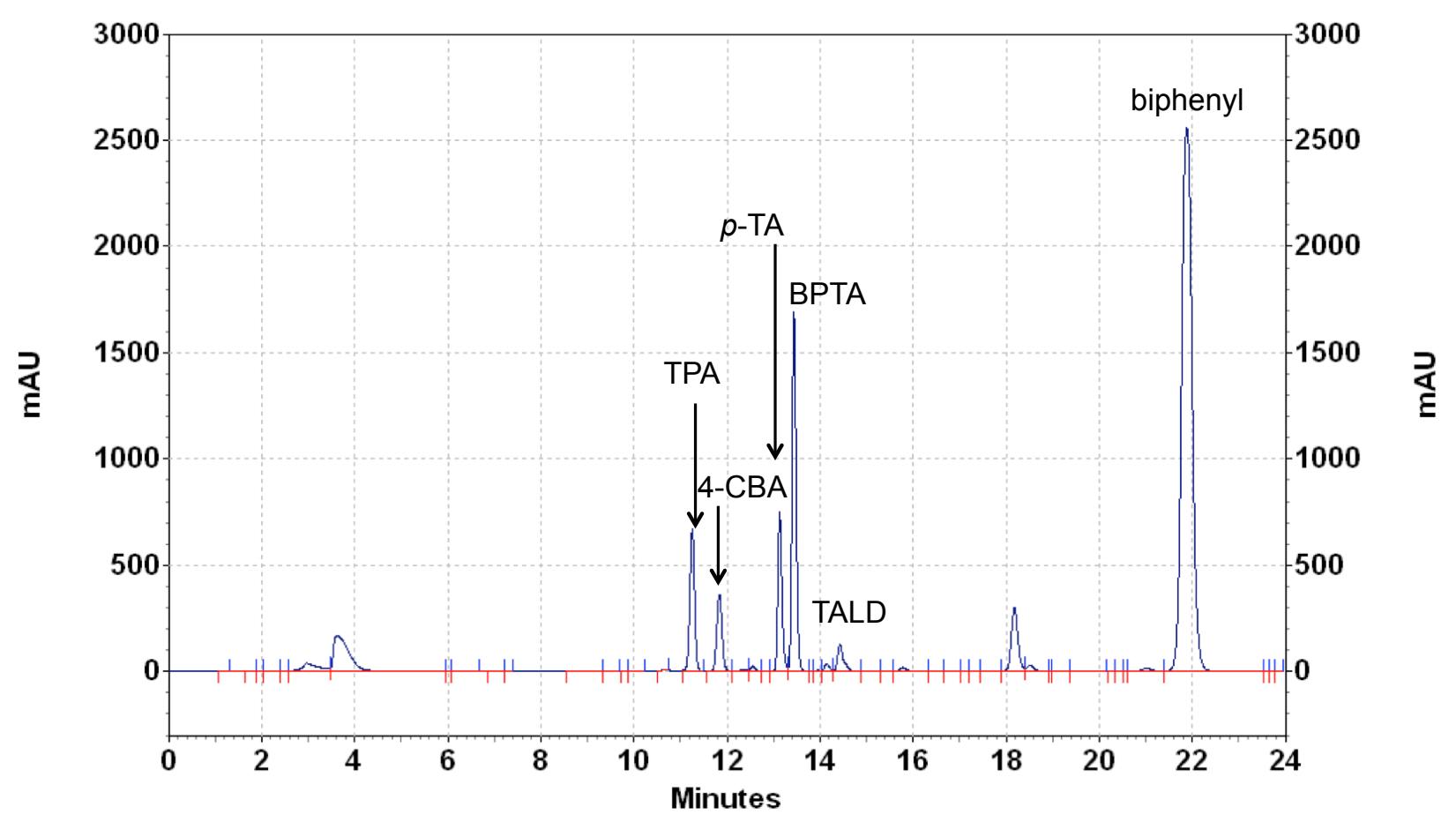

Figure 2.4: Typical HPLC chromatogram of a product sample (after $15 \mathrm{sec}$. of $p \mathrm{X}$ oxidation showing initial product + intermediates)

\subsection{Lumped Kinetic Scheme and Semi-Batch Reactor Model}

None of the reported lumped kinetic models for $p \mathrm{X}$ oxidation to TPA account for 4bromomethyl benzoic acid (BPTA), significant amounts of which are detected in our 
experiments. Considering its possible important role in the process, the formation and disappearance of BPTA are also included in our proposed lumped kinetic scheme as a parallel reaction, as shown in Figure 2.5. BPTA is formed by the substitution reaction involving $p$-TA and $\mathrm{HBr}$, and is oxidized in the presence of oxygen to 4-CBA. The proposed chemical reaction equations associated with the formation and oxidation of BPTA are shown in Figure 2.6.

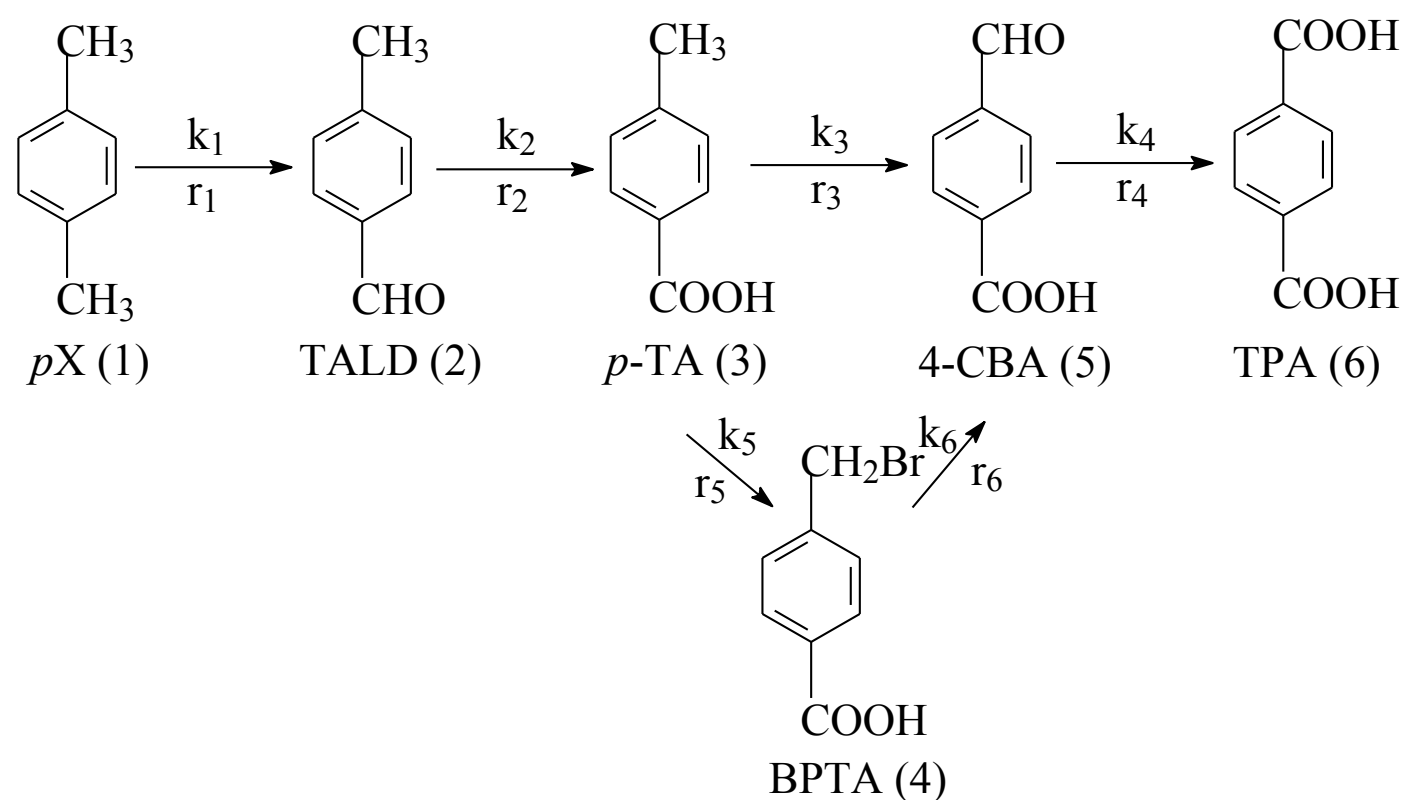

Figure 2.5: Proposed lumped kinetic scheme for $p \mathrm{X}$ oxidation to TPA 

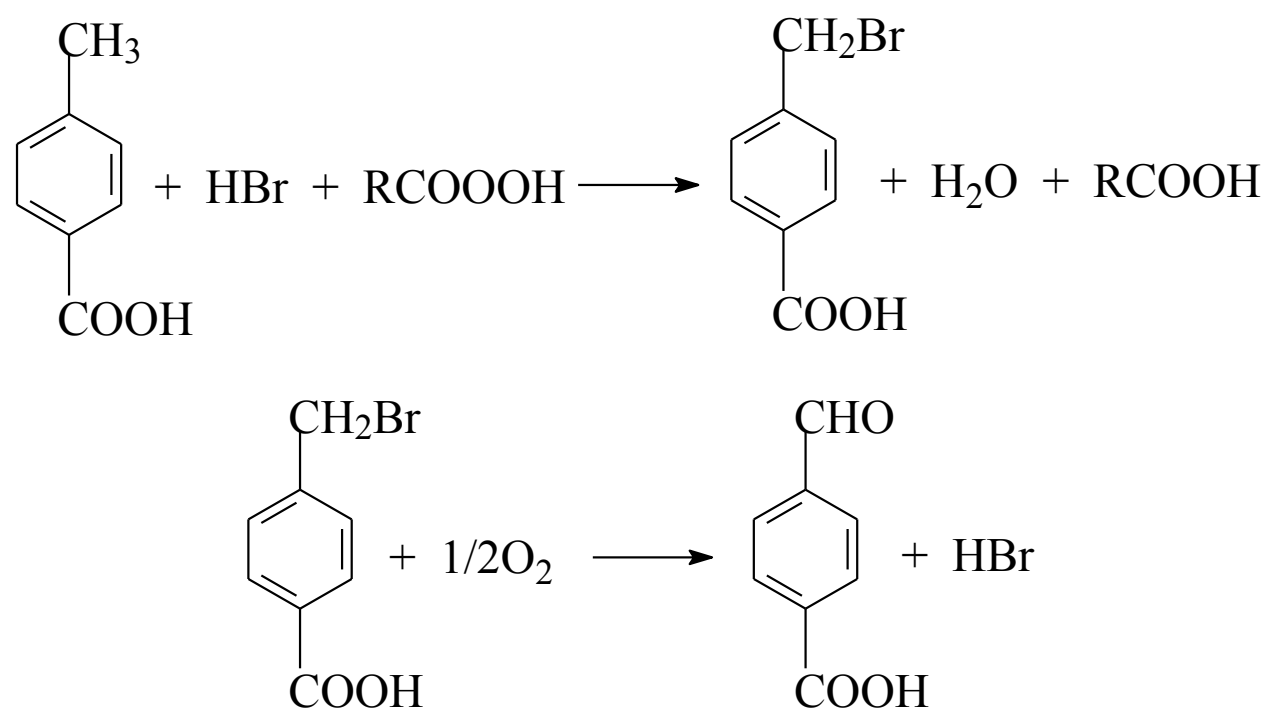

Figure 2.6: Formation and oxidation of BPTA

The reported power law kinetic models for $p \mathrm{X}$ oxidation to TPA mainly assume pseudo-first order oxidation kinetics (in excess of $\mathrm{O}_{2}$ ). Cao et al. [95] applied the first order kinetic model at low $p \mathrm{X}$ conversions wherein no solid phase was formed; i.e., the system was considered homogeneous. Wang et al. [44] also deduced from their fractional model that when the reactant $(p \mathrm{X})$ concentration was low enough compared to $\mathrm{O}_{2}$, the first order kinetic model was valid. In our experiments, we chose to operate at low enough initial $p \mathrm{X}$ concentrations such that even at total conversion to TPA, there would not be any TPA precipitation. Further, the $\mathrm{O}_{2}$ concentrations relative to the $p \mathrm{X}$ concentration was maintained in excess (at least 5-fold). Based on these experimental conditions, we adopted the pseudo-first order kinetic model for the sequential reactions involving $\mathrm{O}_{2}$, with the $\mathrm{O}_{2}$ concentration partial pressure (and therefore its activity in the liquid phase dominated by acetic acid) being maintained constant. The model equations based on these assumptions are given below: 


$$
\begin{aligned}
\frac{d c_{1}}{d t} & =-k_{1} c_{1} \\
\frac{d c_{2}}{d t} & =k_{1} c_{1}-k_{2} c_{2} \\
\frac{d c_{3}}{d t} & =k_{2} c_{2}-k_{3} c_{3}-k_{5} c_{3} \\
\frac{d c_{4}}{d t} & =k_{5} c_{3}-k_{6} c_{4} \\
\frac{d c_{5}}{d t} & =k_{3} c_{3}+k_{6} c_{4}-k_{4} c_{5} \\
\frac{d c_{6}}{d t} & =k_{4} c_{5}
\end{aligned}
$$

with the initial conditions:

$$
c_{1}=c_{10}, c_{2}=c_{3}=c_{4}=c_{5}=c_{6}=0
$$

Athena Visual Studio [103] was used to estimate the kinetic parameters. The non-linear least squares method was employed. The optimization method was implemented in the software and encoded using Fortran. The convergence criteria is based on the evaluation of an experimental variance, $\sigma^{2}$, defined as:

$$
\sigma^{2}=\frac{\sum_{1}^{n}\left(Y_{E}-Y_{P}\right)^{2}}{n-p}<10^{-3}
$$

where $Y_{E}$ and $Y_{P}$ are experimental and predicted functions (in this case, concentration), $n$ is the number of experimental points and $p$ is the number of parameters.

The following observations based on published literature provide valuable guidance for our experimental and modeling studies. It is generally believed that the $p$-TA $\rightarrow$ 4-CBA reaction is the slowest step in the overall oxidation sequence [97, 98]. If this is indeed the case in our experiments, then the estimated first-order rate constant for this step $\left(k_{3}\right)$ should be several-fold less than the other rate constants in the sequential oxidation. The overall oxidation rate would 
then be the rate of the slowest step in the sequence. In such a case, it should be possible to assess the presence or absence of gas-liquid mass transfer limitations associated with the slowest step from the influence of stirrer speed on the estimated rate constant for that step. The estimated rate constants associated with the non-rate determining steps could be merely mass transfer coefficients, recognizing that the $\mathrm{O}_{2}$ transport into the liquid phase is also a first-order rate process.

\subsection{Results and Discussion}

\subsubsection{T \& P profile}

Figure 2.7 shows the temperature and pressure profiles corresponding to run 3 (Table 2.3). Since low enough initial $p \mathrm{X}$ concentration $(0.025 \mathrm{M})$ was charged in the reactor, the reactor temperature rise was within $1{ }^{\circ} \mathrm{C}$ caused by the generated heat of reaction, which allowed us to consider that the reactor was operated under isothermal conditions. The reactor pressure was maintained constant (15 bar) except for brief, insignificant pressure fluctuations during sampling.

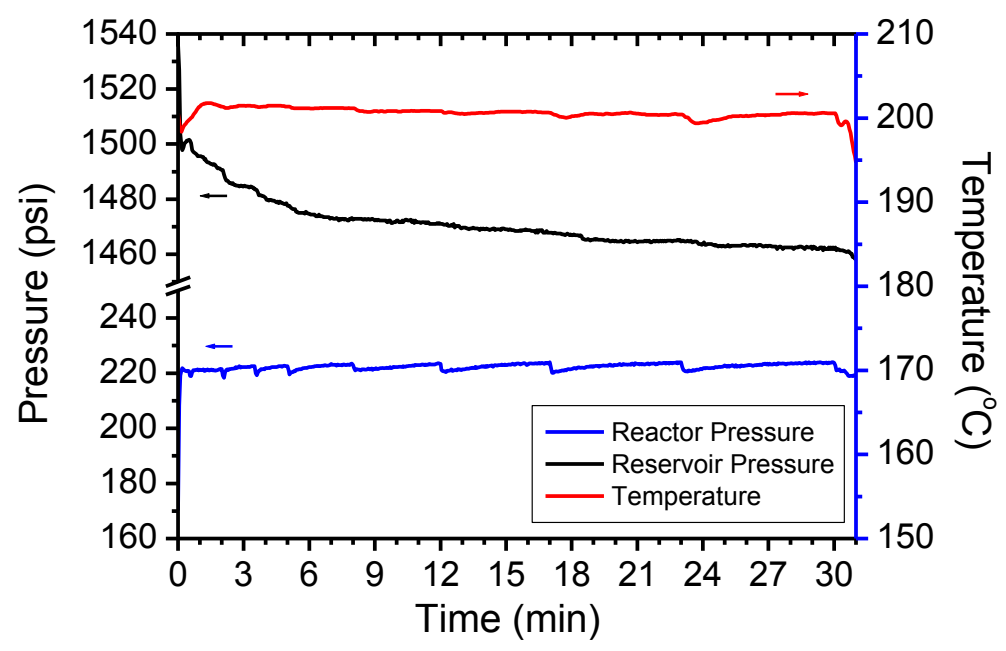

Figure 2.7: Temporal temperature and pressure profiles during intrinsic kinetic studies of $p \mathrm{X}$ oxidation in a stirred reactor 


\subsubsection{Effect of stirring rate}

The intrinsic kinetic rates of the sequential steps during $p \mathrm{X}$ oxidation under MC process conditions $\left(200{ }^{\circ} \mathrm{C}, 15\right.$ bar $)$ are very fast and tend to be limited by gas-liquid mass transfer resistance. The experiments (Runs 1-5 of Table 2.3) were carried out in the stirred reactor to evaluate the effect of stirring speed on the reaction rates. Figure 2.8 shows the temporal product distributions at various stirring speeds. Effective rate constants were obtained from the reactant and product concentration profiles as explained previously.
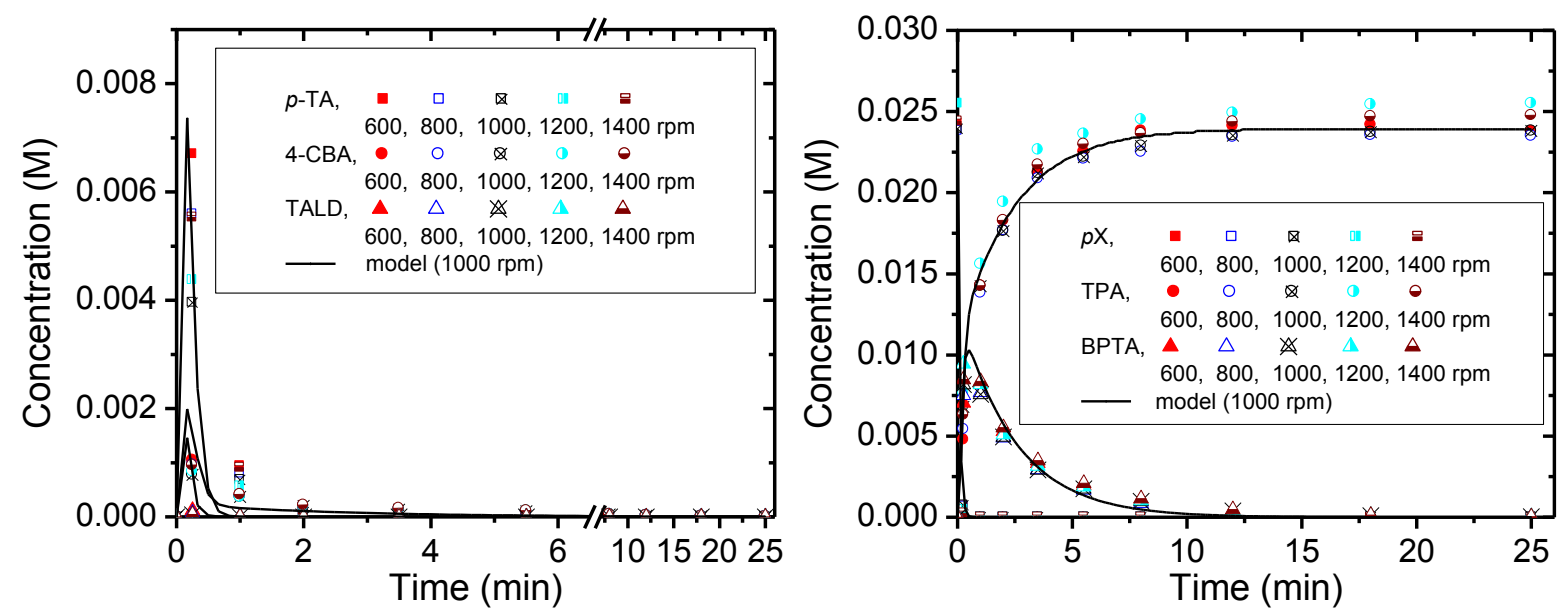

Figure 2.8: Experimental and simulated product distributions at different stirring rates (Reaction conditions: $\mathrm{T}=200{ }^{\circ} \mathrm{C}, \mathrm{P}=15$ bar; Initial $p \mathrm{X}=25 \mathrm{mM}$, Co $=12.5 \mathrm{mM}, \mathrm{Mn}=12.5$ $\left.\mathrm{mM}, \mathrm{Br}=32.5 \mathrm{mM} ; \mathrm{O}_{2}: \mathrm{CO}_{2}(\mathrm{~mol}: \mathrm{mol})=1: 1\right)$

The estimated rate constants are shown in Table 2.4. Clearly, the slowest step in the series reaction sequence is the formation of 4-CBA from $p$-TA, and that in the parallel reaction is the oxidation of BPTA to 4-CBA. At all stirrer speeds ranging from $600-1400 \mathrm{rpm}$, the rate constants associated with these steps $\left(k_{3}\right.$ and $\left.k_{6}\right)$ are several-fold lower than those associated with the other steps. The uncertainties associated with the higher rate constants are greater, as might be anticipated with the measurement of faster reaction rates. For example, because the first two 
reaction rates in the oxidation sequence are so fast, the sampling errors associated with TALD and $p$-TA will be substantially more. This also manifests in the uncertainties of the estimated rate constants. In contrast, the uncertainties associated with the rate constants of the slowest steps $\left(k_{3}\right.$, $k_{5}$ and $k_{6}$ ) are much lower. For the sequential oxidation steps, the rate constant associated with 4CBA formation form $p$-TA $\left(k_{3}\right)$ increases from 600 to $800 \mathrm{rpm}$ (beyond the range of uncertainty) and tends to level off around $1000 \mathrm{rpm}$. In contrast, the other rate constants in the oxidation sequence $\left(k_{1}, k_{2}\right.$ and $\left.k_{4}\right)$ do not vary in this stirrer speed range. These observations suggest that while it is possible to overcome gas-liquid mass transfer limitations associated with the $p$-TA $\rightarrow$ 4-CBA step in the stirred reactor, the kinetics associated with other rates are much faster to eliminate gas-liquid mass transfer limitations. In addition, the lowest rate constant $\left(k_{6}\right)$ is invariant in this stirrer rpm range suggesting that it is not influenced by gas-liquid mass transfer limitations. Note that there is an apparent decrease in rate constants with a further increase in the stirring rate to $1400 \mathrm{rpm}$. This is probably due to the formation of a deep meniscus at the higher agitation speed that decreases the liquid level below that of the sparger (either partly or fully) used for introducing $\mathrm{O}_{2}$ into the reactor. Hence, we conclude that the maximum rate of TPA formation in this stirred reactor occurs beyond $1000 \mathrm{rpm}$ and that it is likely not possible to completely eliminate the mass transfer limitations associated with the formation of the intermediates, TALD and $p$-TA, in a conventional stirred reactor. In other words, the saturation of the liquid phase with oxygen is most likely the rate-limiting step. Any further increase in the overall TPA formation rate must involve another reactor configuration in which the gas-liquid mass transfer limitations associated with the formation of these intermediates are better alleviated. 
Table 2.4: Estimated reaction rate constants at different stirring rates (initial $[p \mathrm{X}]=0.025 \mathrm{M}$; all other experimental conditions are as shown in Table 2.3)

\begin{tabular}{ccccccc}
\hline $\begin{array}{c}\text { Stirring } \\
\text { rate } \\
(\mathrm{rpm})\end{array}$ & $\begin{array}{c}k_{1} \\
\left(\mathrm{~min}^{-1}\right)\end{array}$ & $\begin{array}{c}k_{2} \\
\left(\mathrm{~min}^{-1}\right)\end{array}$ & $\begin{array}{c}k_{3} \\
\left(\mathrm{~min}^{-1}\right)\end{array}$ & $\begin{array}{c}k_{4} \\
\left(\mathrm{~min}^{-1}\right)\end{array}$ & $\begin{array}{c}k_{5} \\
\left(\mathrm{~min}^{-1}\right)\end{array}$ & $\begin{array}{c}k_{6} \\
\left(\mathrm{~min}^{-1}\right)\end{array}$ \\
\hline 600 & $11.7(1.72)^{\mathrm{a}}$ & $33.2(18.9)$ & $3.90(0.548)$ & $17.5(4.78)$ & $4.12(0.568)$ & $0.421(0.0405)$ \\
\hline 800 & $11.8(1.96)$ & $37.1(26.0)$ & $4.45(0.764)$ & $18.6(5.24)$ & $4.71(0.799)$ & $0.423(0.0450)$ \\
\hline 1000 & $11.7(1.85)$ & $39.0(27.1)$ & $5.89(1.10)$ & $22.5(6.83)$ & $6.00(1.10)$ & $0.414(0.0406)$ \\
\hline 1200 & $13.6(2.31)$ & $42.1(32.9)$ & $5.56(1.03)$ & $23.2(6.77)$ & $5.81(1.05)$ & $0.435(0.0364)$ \\
\hline 1400 & $13.9(2.75)$ & $36.8(28.9)$ & $4.49(0.793)$ & $20.5(5.91)$ & $4.88(0.844)$ & $0.401(0.0369)$ \\
\hline
\end{tabular}

${ }^{\mathrm{a}}$ In parenthesis: $95 \%$ confidence level.

Figure 2.8 also shows the experimentally observed concentrations at various stirrer rpms. The fitted concentration profiles at $1000 \mathrm{rpm}$ (solid line) match the experimental observations quite well.

\subsubsection{Comparison of estimated rate constants with literature data}

Table 2.5 compares the estimated reaction rate constants in this work with the reported data from literature. Only those employing the lumped reaction scheme (Figure 1.3) are compared here. Even though different reaction conditions and kinetic models are employed, the relative rate constants of the various steps follow the same trend: $k_{3}<k_{1}<k_{4}<k_{2}$. Our investigations concur with previous reports that the slowest step is the oxidation of $p$-TA to 4-CBA. The estimated reaction rate constants in this work are at least one order of magnitude greater than those reported in the referenced studies. We attribute this to the fact that the reaction was performed under homogeneous conditions in this work, avoiding product precipitation in the liquid phase and thereby eliminating gas-solid and liquid-solid mass transfer limitations. 
Table 2.5: Comparison of estimated rate constants with literature data

\begin{tabular}{ccccccc}
\hline Model type & $\begin{array}{c}k_{1} \\
\left(\mathrm{~min}^{-1}\right)\end{array}$ & $\begin{array}{c}k_{2} \\
\left(\mathrm{~min}^{-1}\right)\end{array}$ & $\begin{array}{c}k_{3} \\
\left(\mathrm{~min}^{-1}\right)\end{array}$ & $\begin{array}{c}k_{4} \\
\left(\mathrm{~min}^{-1}\right)\end{array}$ & Ref. & Remarks \\
\hline $\begin{array}{c}\text { Fractional model } \\
\text { derived from reaction } \\
\text { mechanism }\end{array}$ & 0.176 & 0.725 & 0.0361 & 0.338 & {$[98]$} & \\
\hline $\begin{array}{c}\text { Power law } \\
\text { assumption }\end{array}$ & 0.732 & 1.422 & 0.139 & 0.873 & {$[100]$} & $\begin{array}{c}\text { Parameter re-estimated } \\
\text { for Wang's model }\end{array}$ \\
\hline & 0.38 & 0.99 & 0.123 & 0.44 & {$[99]$} & $\begin{array}{c}0.65 \text {-order to } p \text { X, first- } \\
\text { order to others }\end{array}$ \\
& 11.74 & 39.03 & 5.889 & 22.50 & this work* & Run \# 3 in Table 2.3 \\
\hline
\end{tabular}

*Stirring rate $=1000 \mathrm{rpm}$; initial $[p \mathrm{X}]=0.025 \mathrm{M}$; all other operating conditions listed in Table 2.3 .

\subsubsection{Effect of bromide concentration}

Different bromide concentrations (Runs $3 \& 6-10$ of Table 2.3) were used in the kinetic study experiments for $p \mathrm{X}$ oxidation to TPA to investigate the effect of bromide concentration on the reaction rate. Figure 2.9 shows the color change of the reaction solution corresponding to the various bromide concentrations in Runs $3 \& 6-10$ of Table 3.2, respectively. The solution changes color from blue to pink with bromide concentration from $34.8 \mathrm{mM}$ to $0.91 \mathrm{mM}$. 


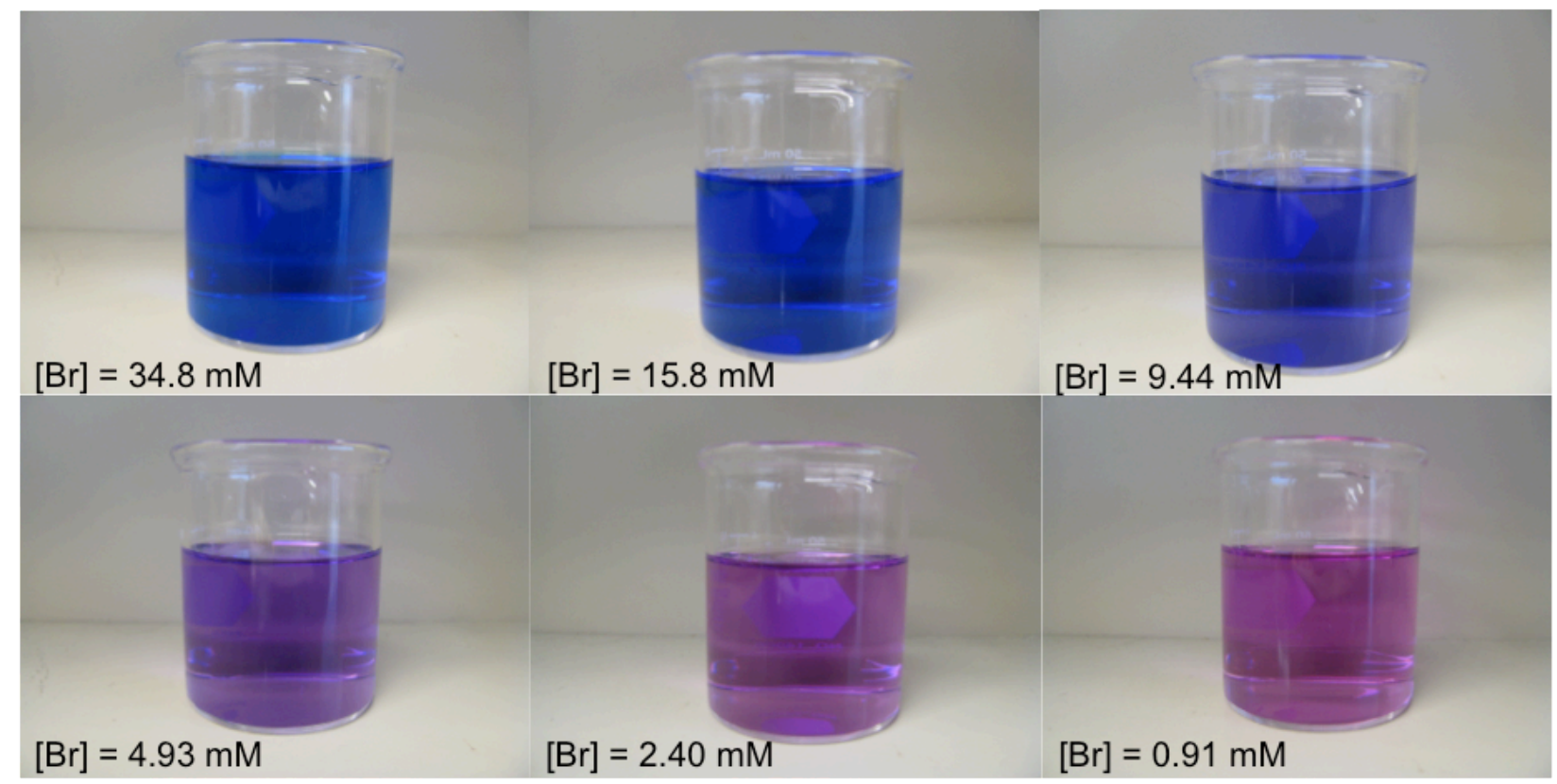

Figure 2.9: Color changes of the reaction solution due to the formation of various cobalt bromides [104] at various bromide concentrations in acetic acid. $[\mathrm{Co}(\mathrm{II})]=12.5 \mathrm{mM}$

Figure 2.10 shows the component concentrations with different bromide concentrations. Surprisingly high concentrations of BPTA were detected during the $p \mathrm{X}$ oxidation. Although it is well recognized that the $p$-TA to 4-CBA conversion is the rate determining step, the slowest step is BPTA oxidation according to the estimated rate constants (Table 2.6). The decrease of the bromide concentration will slow down the BPTA disappearance rate. It is reported as much as $99 \%$ of the initial inorganic bromide is converted to benzylic bromide and its concentration remains about the same until the oxidation of the hydrocarbon is nearly complete [105]. Partenheimer [106, 107], Saha and Espenson [108] also found that a decrease in $\mathrm{Co} / \mathrm{Mn} / \mathrm{Br}$ catalytic activity is attributed to the benzylic bromide formation and benzylic bromide has virtually no activity or promotional effect in $\mathrm{Co} / \mathrm{Mn} / \mathrm{Br}$ autoxidations whereas the inorganic ionic bromide is active. The mechanism of BPTA oxidation has not been well studied. Metelski et al. [105] developed a proposed mechanism for benzyl bromide oxidation (Figure 2.11). According 
to this, benzyl bromide is oxidized in the presence of $\mathrm{Co}(\mathrm{III})$, leading to the recovery of bromide ions. Simultaneously, $\mathrm{Co}(\mathrm{III})$ is reduced to $\mathrm{Co}(\mathrm{II})$ by hydrogen bromide (faster) and also by benzyl bromide, albeit at a much slower rate. Partenheimer [106] reported that the competing mechanisms of oxidation and solvolysis for the disappearance of benzylic bromide occur at approximately the same rates under the studied experimental conditions.
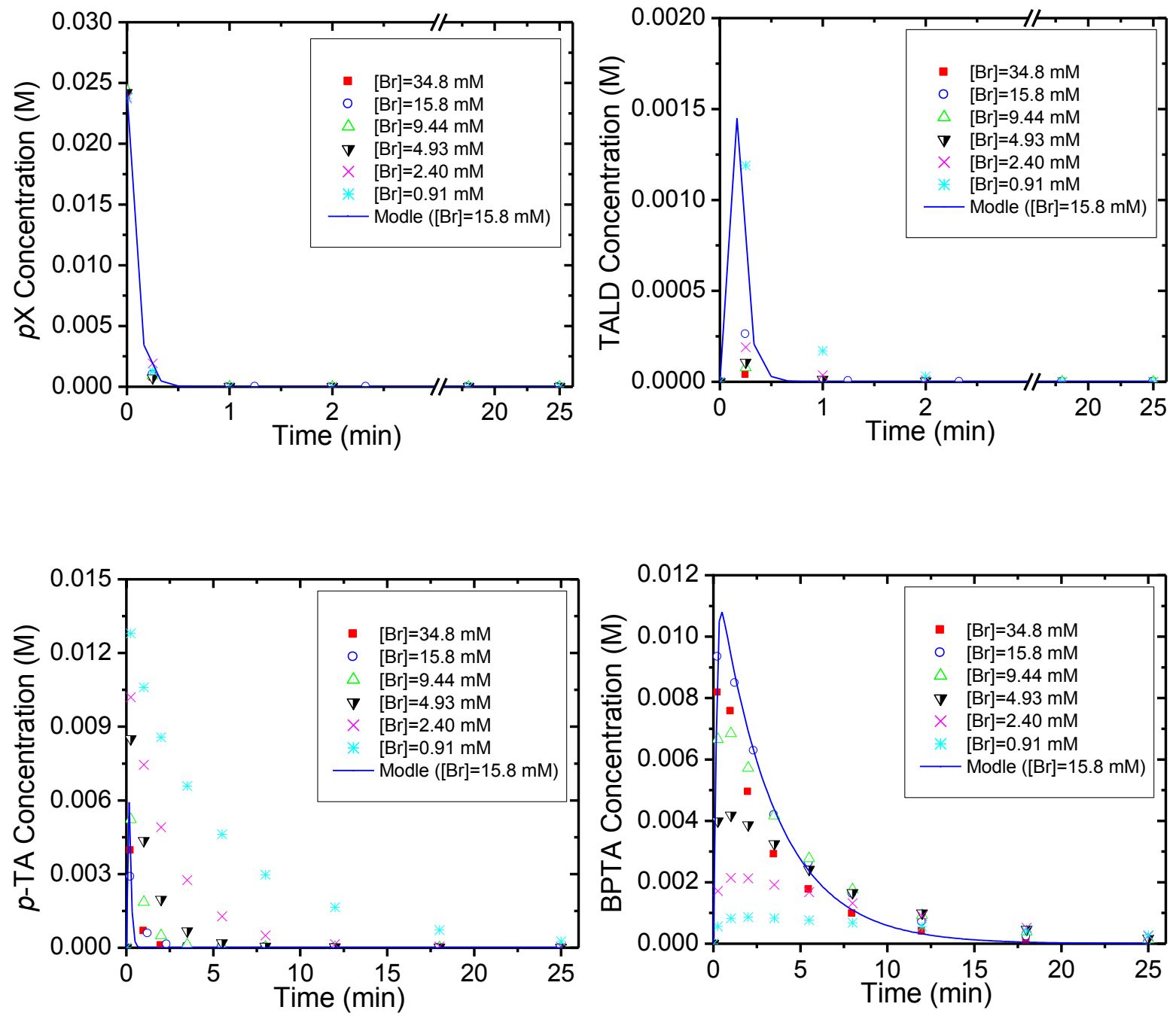

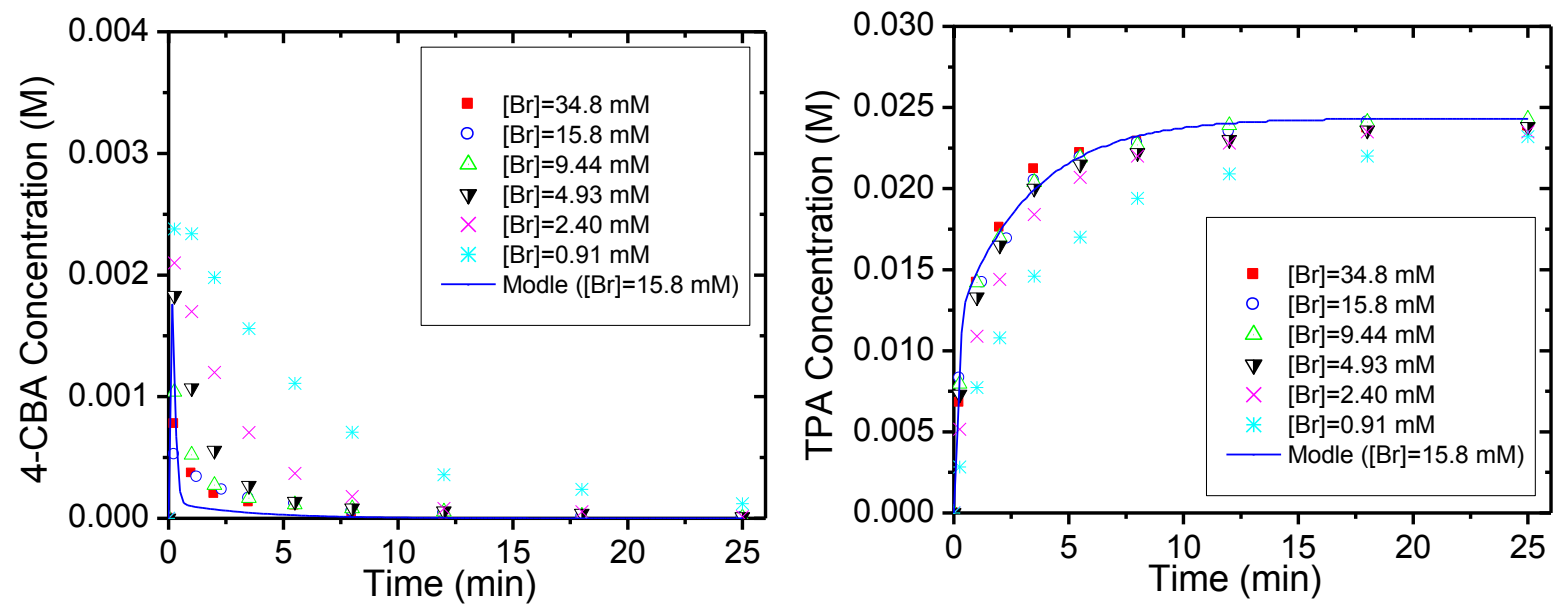

Figure 2.10: Experimental and simulated product distributions with various bromide concentrations (initial $[p \mathrm{X}]=0.025 \mathrm{M}$; stirring rate $=1000 \mathrm{rpm}$; all other experimental conditions are as shown in Table 2.3)

Table 2.6: Estimated reaction rate constants at different bromide concentrations (initial $[p \mathrm{X}]=$ $0.025 \mathrm{M}$; stirring rate $=1000 \mathrm{rpm}$; all other experimental conditions are as shown in Table 2.3)

\begin{tabular}{ccccccc}
\hline $\begin{array}{c}\mathrm{C}_{\mathrm{Br}} \\
(\mathrm{mM})\end{array}$ & $\begin{array}{c}k_{1} \\
\left(\mathrm{~min}^{-1}\right)\end{array}$ & $\begin{array}{c}k_{2} \\
\left(\mathrm{~min}^{-1}\right)\end{array}$ & $\begin{array}{c}k_{3} \\
\left(\mathrm{~min}^{-1}\right)\end{array}$ & $\begin{array}{c}k_{4} \\
\left(\mathrm{~min}^{-1}\right)\end{array}$ & $\begin{array}{c}k_{5} \\
\left(\mathrm{~min}^{-1}\right)\end{array}$ & $\begin{array}{c}k_{6} \\
\left(\mathrm{~min}^{-1}\right)\end{array}$ \\
\hline 34.8 & $11.7(1.91)^{\mathrm{a}}$ & $39.0(27.1)$ & $5.89(1.10)$ & $22.5(6.83)$ & $6.00(1.10)$ & $0.414(0.0405)$ \\
15.8 & $11.8(1.60)$ & $39.6(25.1)$ & $7.75(1.45)$ & $29.3(10.3)$ & $7.69(1.42)$ & $0.307(0.0227)$ \\
9.44 & $11.6(2.28)$ & $45.6(43.2)$ & $5.64(1.27)$ & $25.4(12.8)$ & $4.17(0.955)$ & $0.261(0.0359)$ \\
4.93 & $8.25(2.36)$ & $56.3(48.2)$ & $2.43(0.648)$ & $11.8(6.51)$ & $1.17(0.413)$ & $0.205(0.0913)$ \\
2.40 & $6.45(1.70)$ & $17.6(11.0)$ & $0.927(0.183)$ & $7.84(4.08)$ & $0.223(0.119)$ & $0.152(0.125)$ \\
0.91 & $7.80(2.66)$ & $14.5(8.92)$ & $0.419(0.0719)$ & $3.87(1.93)$ & $0.0585(0.0477)$ & $0.0658(0.0599)$ \\
\hline
\end{tabular}

${ }^{a}$ In parenthesis: $95 \%$ confidence level. 

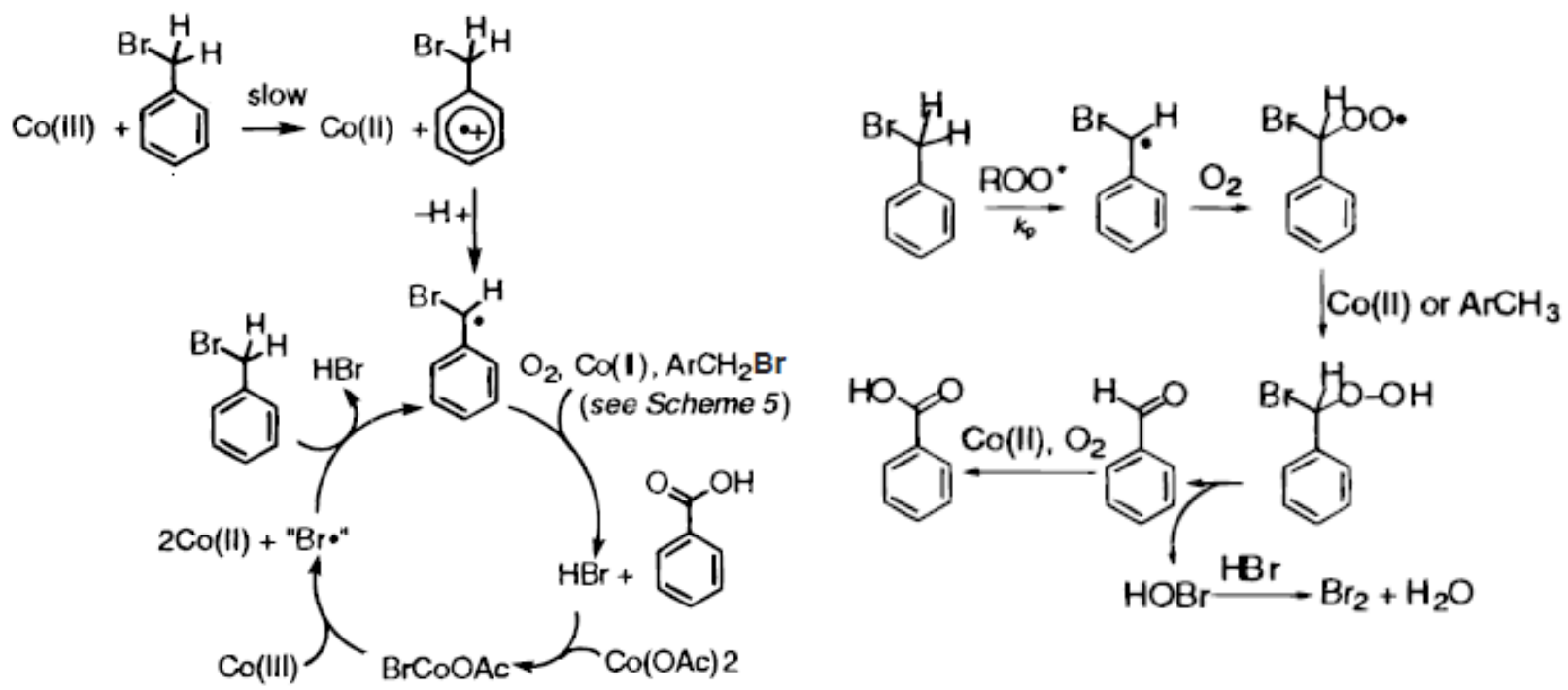

Figure 2.11: Proposed Scheme for the autoxidation of benzyl bromide [105]

\subsubsection{Effect of substrate concentration}

Experiments with different $p \mathrm{X}$ concentrations (Runs $6,11 \& 12$ of Table 2.3) were carried out to study the effect of substrate concentration on the regressed rate constants. Table 2.7 shows the estimated rate constants. It was found that the rate constants at the different concentrations are invariant when the confidence intervals are taken into account. This invariance is to be expected in the case of intrinsic rate constants. However, as shown in Figure 2.12, the rate constants are reported to decrease with the increase of initial $p \mathrm{X}$ concentration in a three-phase reaction system [99]. This suggests that the formation of a solid phase introduces mass transfer resistances that lower the rates and therefore the rate constants. 
Table 2.7: Estimated reaction rate constants at different $p X$ concentrations $(\mathrm{P}=15$ bar; $\mathrm{T}=200$ $\left({ }^{\circ} \mathrm{C}\right.$; stirring rate $=1000 \mathrm{rpm}$; all other experimental conditions are as shown in Table 2.3)

\begin{tabular}{cccccccc}
\hline Run \# & $\begin{array}{c}\mathrm{C}_{p X, 0} \\
(\mathrm{mM})\end{array}$ & $\begin{array}{c}k_{1} \\
\left(\mathrm{~min}^{-1}\right)\end{array}$ & $\begin{array}{c}k_{2} \\
\left(\mathrm{~min}^{-1}\right)\end{array}$ & $\begin{array}{c}k_{3} \\
\left(\mathrm{~min}^{-1}\right)\end{array}$ & $\begin{array}{c}k_{4} \\
\left(\mathrm{~min}^{-1}\right)\end{array}$ & $\begin{array}{c}k_{5} \\
\left(\mathrm{~min}^{-1}\right)\end{array}$ & $\begin{array}{c}k_{6} \\
\left(\mathrm{~min}^{-1}\right)\end{array}$ \\
\hline 12 & 14 & $11.8(2.56)^{\mathrm{a}}$ & $40.1(42.0)$ & $5.20(1.27)$ & $18.73(6.60)$ & $5.64(1.37)$ & $0.225(0.0255)$ \\
6 & 25 & $11.8(1.60)$ & $39.6(25.1)$ & $7.75(1.45)$ & $29.3(10.3)$ & $7.69(1.42)$ & $0.307(0.0227)$ \\
11 & 35 & $11.0(2.10)$ & $38.9(29.9)$ & $8.81(2.36)$ & $30.3(16.9)$ & $5.87(1.61)$ & $0.302(0.0445)$ \\
\hline
\end{tabular}

${ }^{\mathrm{a}}$ In parenthesis: 95\% confidence level.
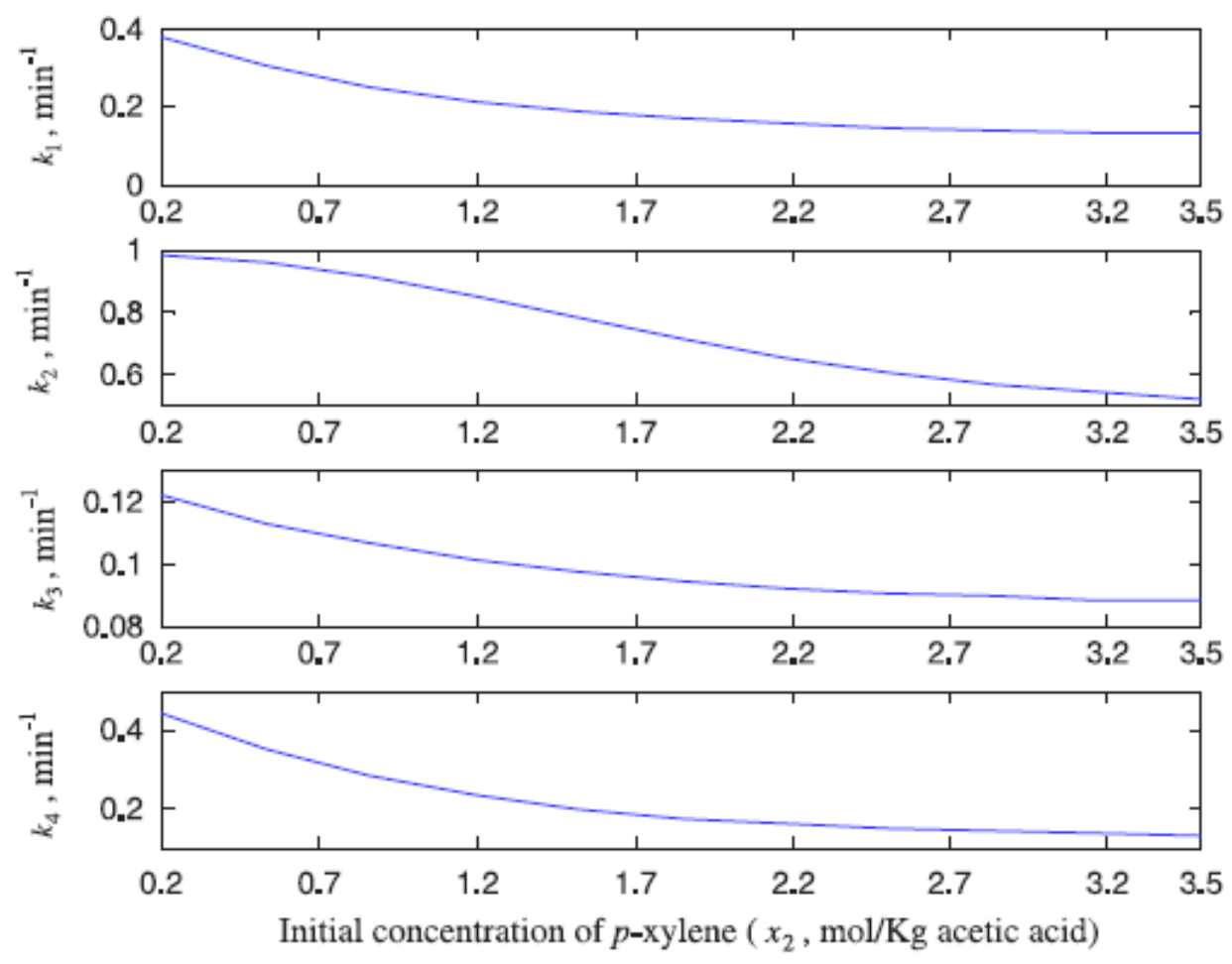

Figure 2.12: Effect of initial concentration of $p \mathrm{X}$ on the reaction rate [99]

\subsection{Summary}

In order to provide guidelines for the rational design and optimization of the spray process for TPA production as well as reactor modeling studies, the kinetic study of $p \mathrm{X}$ liquid phase oxidation to TPA was investigated in a stirred reactor under homogeneous conditions (i.e., low $p \mathrm{X}$ concentrations employed to avoid TPA precipitation). Solubility data of MC process related 
components such as TPA, 4-CBA and $p$-TA in acetic acid over a wide temperature range were obtained experimentally and verified with reported data. Based on the solubility data, appropriate reaction conditions were determined for investigating the kinetics of the oxidation reaction. A first order kinetic model was developed based on a lumped reaction scheme that includes a parallel step describing the formation of 4-(bromomethyl)benzoic acid (BPTA). Kinetic rate constants regressed from the experimental data are shown to be at least one order of magnitude greater than those reported in the literature. This is attributed to the fact that the conversion data were operated under homogeneous conditions in this study (unlike the literature data that were obtained in gas-liquid-solid systems) avoiding solid formation in the liquid phase and thereby eliminating gas-solid and liquid-solid mass transfer limitations. Our investigations concur with previous reports that the slowest step is the oxidation of $p$-TA to 4-CBA. Systematic investigations of the effects of stirring rate reveal that it is not possible to completely eliminate the gas-liquid mass transfer limitations in even a laboratory scale stirred reactor. This further confirms that other reactor configurations (such as a spray reactor in which the liquid is the dispersed phase) are needed to overcome gas-liquid mass transfer limitations. The effects of bromide and initial $p \mathrm{X}$ concentrations on the reaction rate were also investigated. Bromide concentration accelerates the reaction rate significantly up to a certain concentration level and shows zero order dependence at high concentrations where the intermediate BPTA elimination is favored. Decreasing reaction rate constants with an increase of initial $p \mathrm{X}$ concentration reported in traditional three-phase reaction systems were not observed in the homogeneous system, which further confirms that the solid phase hinders the reaction rate and the literature reported kinetic rate constants are not intrinsic kinetic parameters. 


\section{Chapter 3 A Spray Reactor Concept for Catalytic Oxidation of $p$-Xylene to Produce High-Purity Terephthalic Acid}

Knowledge of intrinsic kinetics and the type of internal contacting pattern employed for the reactants are key factors among many that dictate chemical reactor performance (Figure 3.1). Kinetics provides information on the speed of the various steps in the overall reaction as a function of reactant concentrations and temperature. The contacting pattern determines how materials flow through and contact each other in the reactor, how early or late they mix, their clumpiness or state of aggregation [109].

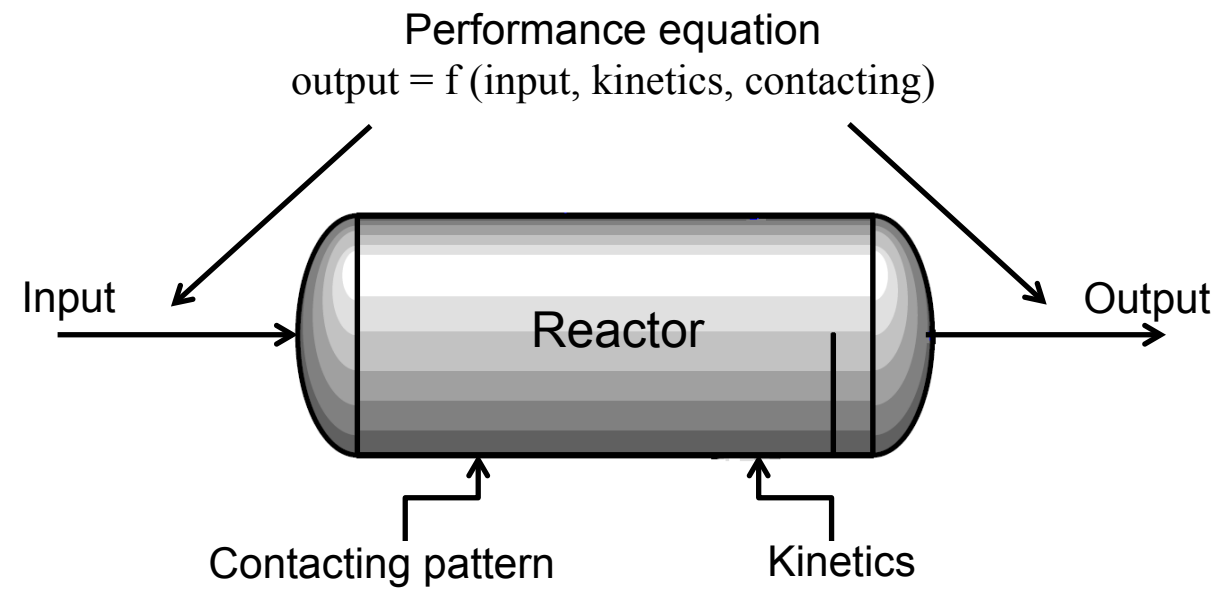

Figure 3.1: Factors that dictate reactor performance [109]

Kinetic studies of liquid phase $p \mathrm{X}$ oxidation to TPA in the previous chapter reveal that it is not possible to completely eliminate the gas-liquid mass transfer limitations in even a laboratory scale stirred reactor. Any further increase in the overall TPA formation rate must involve another reactor configuration in which the gas-liquid mass transfer limitations associated with the 
formation of these intermediates are better alleviated. This is the issue of internal contacting pattern of the reactants in the reactor configuration.

Contacting pattern is especially important in the case of gas-liquid reaction systems, due primarily to possible gas-liquid mass transfer resistance. The commercial MC process for TPA production employs either a CSTR or a bubble column reactor where air is bubbled through the liquid phase. Given that the rates of the sequential oxidation reaction steps are extremely rapid, the presence of gas-liquid mass transfer limitations will cause $\mathrm{O}_{2}$ starvation in the liquid phase resulting in incomplete oxidation. A number of patents deal with improving the agitation system to maximize the $\mathrm{O}_{2}$ /liquid mass transfer coefficient, thereby improving the oxidation rates and obtaining a purer TPA product $[110,111]$. In addition, Osada and Savage [112] found that the oxygen introduction method has a profound influence on the course of $p \mathrm{X}$ partial oxidation reaction in high-temperature water as reaction medium. Adding oxygen in quick, discrete, small incremental bursts led to dramatically higher yields and selectivities of TPA than continuous addition of oxygen. They hypothesized that the rapid step-wise $\mathrm{O}_{2}$ introduction provided additional turbulence and better mixing as well as higher local $\mathrm{O}_{2}$ concentrations compared to steady introduction of an identical amount of $\mathrm{O}_{2}$ in a given time. The authors suggested that an optimal reactor configuration is a tubular reactor in which a stream of high-temperature water containing dissolved catalyst and substrate $(p X)$ is flowed with intermittent introduction of oxygen bursts through side-stream jets placed along the tube length.

This chapter describes a spray reactor concept with a different gas/liquid contacting pattern compared to the conventional MC process reactor for TPA production. A theoretical basis guide by reactor engineering fundamentals is provided to justify this contacting pattern and to rationally design the spray reactor. A mathematical model of the conventional reactor provides 
valuable insights into how effective temperature control and safe operation of the exothermic oxidation system are achieved in industrial practice. These insights provide rational guidance for experimental demonstration of safe operation of a laboratory scale spray reactor whose performance is compared with a stirred tank reactor with respect to product yield and purity.

\subsection{Theoretical Considerations}

\subsubsection{Rationale for choosing spray reactor as an alternative to a stirred reactor}

At the operating temperature of the MC process, the gas phase containing the oxidant is dispersed through the liquid phase containing the substrate $(p \mathrm{X})$. The TPA formation involves a series of partial oxidation steps in which the methyl groups in $p \mathrm{X}$ are sequentially oxidized to yield TALD, $p$-TA, 4-CBA and finally TPA. However, the oxidation rates are so fast such that, even if the $\mathrm{O}_{2}$ in the bulk gas phase is present in stoichiometric amounts for TPA formation, the overall conversion is limited by severe gas-to-liquid mass transfer limitations. This in turn causes $\mathrm{O}_{2}$ starvation in the bulk of the liquid phase containing the substrate and the intermediate oxidation products. If one envisions spherical droplets, the outermost liquid layer in each droplet that is in contact with the oxygen will be easily oxidized causing the TPA to precipitate with incomplete oxidation products trapped in the inner core of the particles. In order to overcome the $\mathrm{O}_{2}$-to-liquid mass transfer limitations, we conceptualized a spray-reactor concept in which the liquid phase is dispersed as micron sized droplets into a continuous gas phase containing the oxidant (oxygen). Each of these droplets may be envisioned to function as a microreactor. In this manner, the gas-liquid interfacial area is significantly enhanced relative to the stirred reactor in which the gas is the dispersed phase.

If the oxygen surrounding each droplet is able to completely penetrate the droplet [i.e., if the

$\mathrm{O}_{2}$ diffusion time scale $\left(R_{\mathrm{p}}{ }^{2} / D\right)$ is shorter than the kinetic time scale $\left.(1 / k)\right]$, then the oxidation 
should progress uniformly throughout the droplet. Such a scenario provides the potential for complete $p$-xylene conversion to produce the ultimate product (TPA) at relatively high purity. In contrast, if the droplet size is large such that the oxygen diffusion time scale is much longer than the kinetic time scale, the resulting gradient in the $\mathrm{O}_{2}$ concentration within the droplet could give rise to non-uniform oxidation of the $p$-xylene leading to a less pure TPA product contaminated by intermediates, lower product yield and quality. Clearly therefore, smaller droplets should favor the production of TPA product with higher purity and yield.

Table 3.1 provides quantitative estimates of the $\mathrm{O}_{2}$ diffusion time scale $\left(R_{\mathrm{p}}{ }^{2} / D\right)$ and the kinetic time scale $(1 / k)$. For this estimation, we assumed an average droplet size of $50 \mu \mathrm{m}$ that is typical for the nozzles (BETE PJ6, BETE Fog Nozzle, Inc.) employed in our experiments. The $\mathrm{O}_{2}$ diffusivity $(D)$ in acetic acid (the dominant specie in the droplet) at $200{ }^{\circ} \mathrm{C}$ and 15 bar is estimated from the Wilke-Chang correlation [113]. The first-order rate constant ( $k$ ) corresponds to the slowest step in the consecutive oxidation sequence (typically $p$-TA oxidation to 4-CBA). Values reported in the literature [94-99] vary widely depending on the catalyst and operating conditions used. We used all these values as well as the rate constants determined in this work (Chapter 2) to estimate the square of Hatta number (Hatta number, $\mathrm{M}_{\mathrm{H}}$, is defined by eqn. 3.1 [109]) for this gas-liquid reaction at various operating conditions as summarized in Table 3.1. The fact that the square of Hatta number is far less than unity at all the conditions allows us to conclude that it is possible to eliminate $\mathrm{O}_{2}$ starvation in typical spray droplets.

$$
\mathrm{M}_{\mathrm{H}}^{2}=\frac{\text { max possible conversion in film }}{\text { max diffusional transport through the film }}
$$


Table 3.1: Estimation of Hatta number for simultaneous $\mathrm{O}_{2}$ diffusion and reaction in a droplet

\begin{tabular}{|c|c|c|c|}
\hline Operating conditions & $\begin{array}{c}k \\
\left(\min ^{-1}\right)\end{array}$ & $\begin{array}{l}k R_{\mathrm{p}}^{2} / D \\
\text { (analogous to } \\
\text { Hatta number } \\
\text { square) }\end{array}$ & $\begin{array}{l}\text { Reference } \\
\quad(\text { for } k)\end{array}$ \\
\hline $\begin{array}{l}\mathrm{T}=130{ }^{\circ} \mathrm{C}, \mathrm{P}=1 \mathrm{~atm} \\
C_{p \mathrm{X}, 0}=3.8 \mathrm{~mol} / \mathrm{kg}_{\text {sol }} \\
C_{\text {catalyst (cobalt naphtenate) }}=0.001 \mathrm{~mol} / \mathrm{kg}_{\text {sol }}\end{array}$ & $6.6\left(10^{-4}\right)$ & $2.3\left(10^{-7}\right)$ & 94 \\
\hline $\begin{array}{l}\mathrm{T}=191{ }^{\circ} \mathrm{C}, \mathrm{P}_{\mathrm{O} 2}=40 \mathrm{kPa} \\
C_{p \mathrm{X}, 0}=0.943 \mathrm{~mol} / \mathrm{kg}_{\mathrm{HAc}} \\
C_{\mathrm{Co}}=0.055 \mathrm{~kg} / \mathrm{kg}_{\mathrm{HAc}}, C_{\mathrm{Mn}}=0.0512 \mathrm{~kg} / \mathrm{kg}_{\mathrm{HAc}} \\
C_{\mathrm{Br}}=0.0746 \mathrm{~kg} / \mathrm{kg}_{\mathrm{HAc}}\end{array}$ & 0.0568 & $2.0\left(10^{-5}\right)$ & 98 \\
\hline $\begin{array}{l}\mathrm{T}=187^{\circ} \mathrm{C} \\
C_{p \mathrm{X}, 0}=0.2 \mathrm{~mol} / \mathrm{kg}_{\mathrm{HAc}} \\
C_{\mathrm{Co}}=0.04 \mathrm{wt} \%, C_{\mathrm{Mn}}=0.02 \mathrm{wt} \%, C_{\mathrm{Br}}=0.08 \mathrm{wt} \%\end{array}$ & 0.123 & $4.3\left(10^{-5}\right)$ & 99 \\
\hline $\begin{array}{l}\mathrm{T}=200{ }^{\circ} \mathrm{C} \\
C_{p X, 0}=0.025 \mathrm{M} \\
C_{\mathrm{Co}}=12.5 \mathrm{mM}, C_{\mathrm{Mn}}=12.5 \mathrm{mM}, C_{\mathrm{Br}}=16 \mathrm{mM}\end{array}$ & 7.75 & $2.7\left(10^{-3}\right)$ & This work \\
\hline
\end{tabular}

\subsubsection{Droplet/reactor stability considerations}

The oxidation of $p \mathrm{X}$ to TPA is highly exothermic with $\Delta H_{R}=1,300 \mathrm{~kJ} / \mathrm{mol}[37,38]$. The droplet temperature rise due to reaction is partly controlled by the evaporative cooling of the solvent (acetic acid). At typical operating conditions, the latent heat of vaporization provides enough heat capacity to absorb the heat of reaction and maintain the maximum droplet temperature at the acetic acid boiling point. As shown in Figure 3.2, the heat of vaporization of acetic acid decreases and the boiling point increases with an increase in pressure. Clearly, proper choice and control of the operating pressure are essential to limit the droplet temperature rise to the boiling point of acetic acid and thereby prevent temperature overshoot that can lead to undesired reactions (burning of acetic acid, for example) and a thermal runaway situation. 


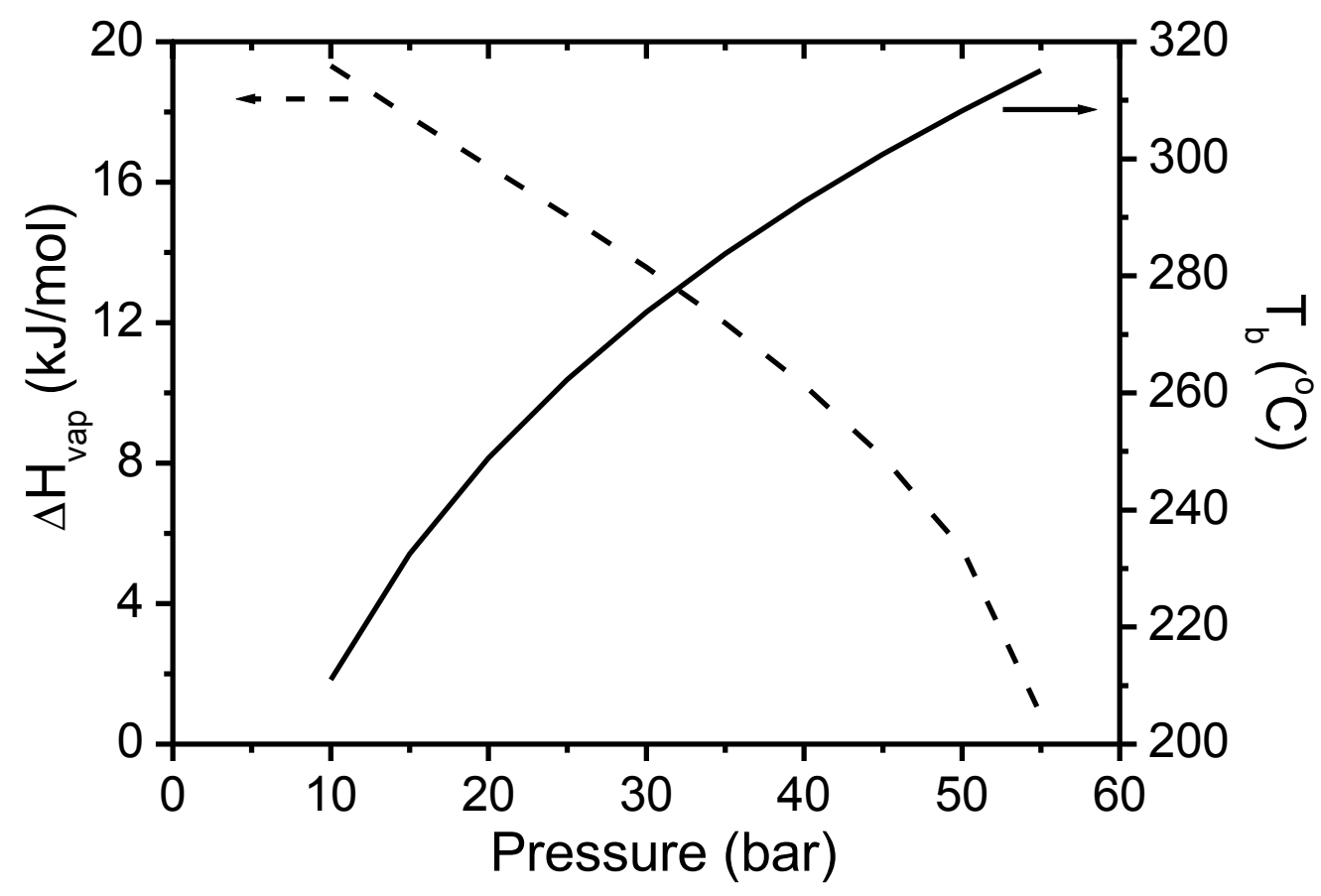

Figure 3.2: Variation of acetic acid boiling point and heat of vaporization with pressure

To better understand temperature control by evaporative cooling, we chose to mathematically model the continuous stirred tank reactor configuration (Figure 3.3) in the MC process. In addition to the feed and recycle streams, the latent heat of evaporation of the mixed solvent (acetic acid and water) at the reactor $\mathrm{P}$ and $\mathrm{T}$ is also a potential coolant source. Note in Figure 3.3 that the acetic acid and water vapor steams are condensed in an external separator and recycled back to the reactor. Based on these assumptions, the energy balance equation for the oxidation reactor is given by:

$$
\sum_{j=1}^{n} F_{j_{0}}\left(H_{j_{0}}-H_{j}\right)+\frac{r_{i} V}{v_{i}}\left(-\Delta H_{R}\right)=\sum_{j=1}^{n} N_{j} C_{p, j} \frac{d T}{d t}
$$


The first term of the left hand side of eqn. 3.2 represents the heat removal rate $\left(-Q_{R}\right)$ and the second term represents the heat generation rate $\left(Q_{G}\right)$. These individual terms are further elaborated in eqns. 3.3 and 3.4 .

$$
\begin{aligned}
& \sum_{j=1}^{n} F_{j_{0}}\left(H_{j_{0}}-H_{j}\right)=-Q_{R}=\sum_{j=1}^{n} F_{j_{0}} \int_{T}^{T_{j_{0}}} C_{p, j} d T-\sum_{j=1}^{m} F_{j_{u a p}} \Delta H_{v a p, j} \\
& =F_{\text {feed }} C_{p, \text { feed }}\left(T_{0}-T\right)+F_{\text {air }} C_{p, \text { air }}\left(T_{0}-T\right) \\
& +F_{\text {HAc, condensed }} C_{p, H A c}\left(T_{\text {recycle }}-T\right)+F_{H_{2} O \text {,condensed }} C_{p, H_{2} O}\left(T_{\text {recycle }}-T\right) \\
& -F_{H A c, v a p} \Delta H_{v a p, H O A c}-F_{H_{2} O, v a p} \Delta H_{v a p, H_{2} O} \\
& \frac{r_{i} V}{v_{i}}\left(-\Delta H_{R}\right)=Q_{G}=F_{p X} X\left(-\Delta H_{R}\right)
\end{aligned}
$$

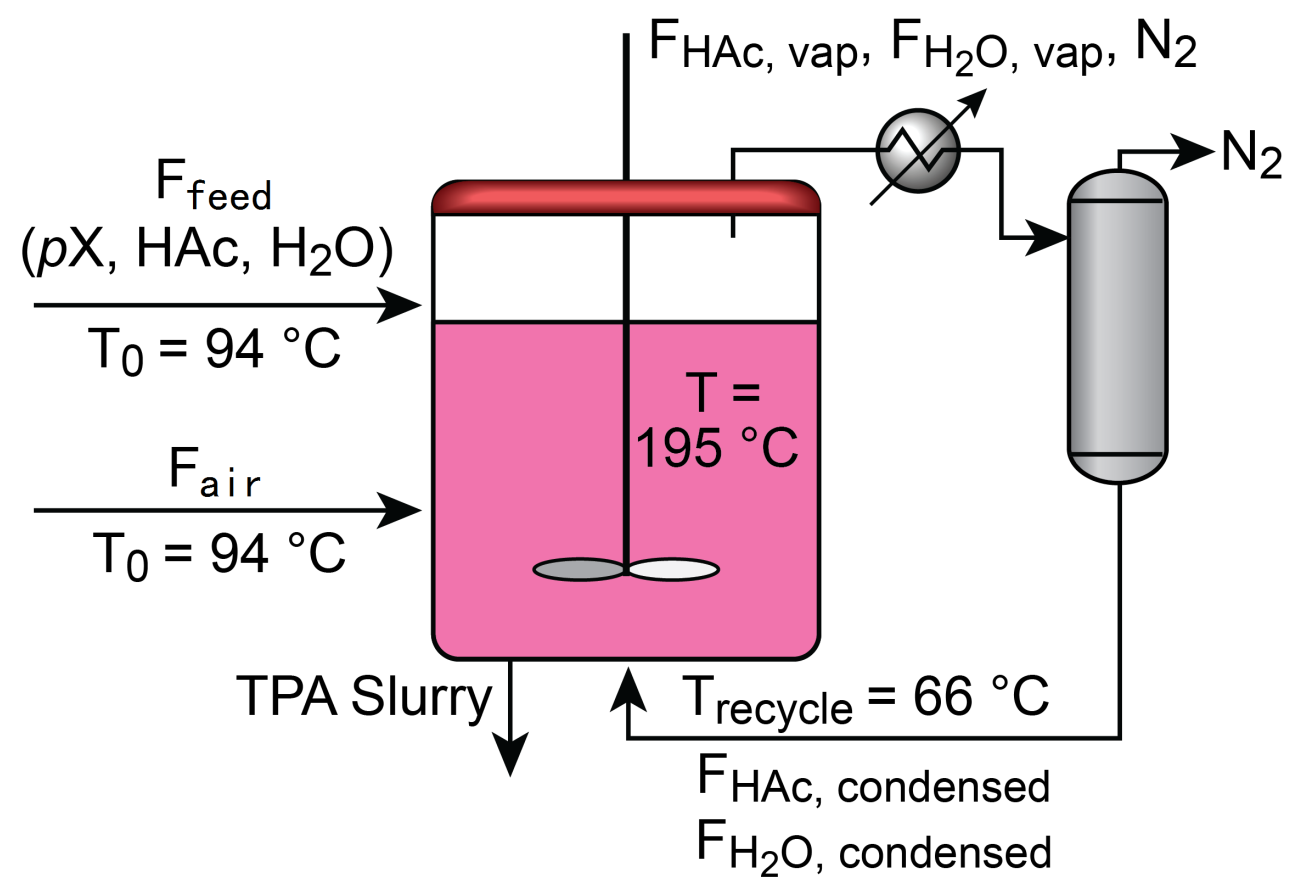

Figure 3.3: Schematic of oxidation reactor at typical MC process conditions $[13,14,20]$

For this analysis, the exothermic heat effects from the intermediate oxidation products (TALD, $p$-TA, 4-CBA) and from solvent burning are neglected as being insignificant compared to the heat effects associated with the predominant TPA formation reaction. Since the $p \mathrm{X}$ 
conversion is complete under typical MC process conditions (with TPA being the dominant product), the heat generation rate is constant at all simulated conditions. The physical property data required to evaluate the various enthalpies in eqn. 3.3 are taken from HYSYS ${ }^{\circledR}$ database [37] and other sources [38]. The vapor phase compositions in the reactor at various $\mathrm{T}$ and fixed reactor P are also estimated using the HYSYS ${ }^{\circledR}$ database.

Figure 3.4 shows the heat generation rate $\left(Q_{G}\right)$ and heat removal rate $\left(Q_{R}\right)$ as a function of temperature for the $\mathrm{MC}$ process oxidation reactor at two different reactor operating pressures: 15.2 bar and 12.5 bar. Note the exponential increase in $Q_{R}$ as the boiling point of the acetic acid (dominant component in the reaction mixture) is approached at the two pressures. At the typical MC process operating pressure of 15.2 bar, the steady state temperature (where $Q_{G}=Q_{R}$ ) is approximately $195{ }^{\circ} \mathrm{C}$. This is remarkably close to the steady state operating temperature reported for industrial reactors $[13,14]$. The evaporative cooling effect accounts for $65 \%$ of the total heat removed. As seen in Figure 3.4, the steady state temperature decreases as pressure decreases. This is because the boiling point of the solvent (acetic acid/water is a tangentially zeotropic system [114]) decreases while the latent heat of evaporation increases with pressure. Further, the slope of heat removal curve is greater than that of heat generation curve, implying that the steady states are open-loop stable. This analysis should clearly hold even when the dispersed and continuous phases are switched: i.e., when the liquid phase exists as droplets (i.e., as microreactors) surrounded by a continuous gas phase containing the oxidant, implying that a spray reactor operated under similar conditions should also be open-loop stable. 


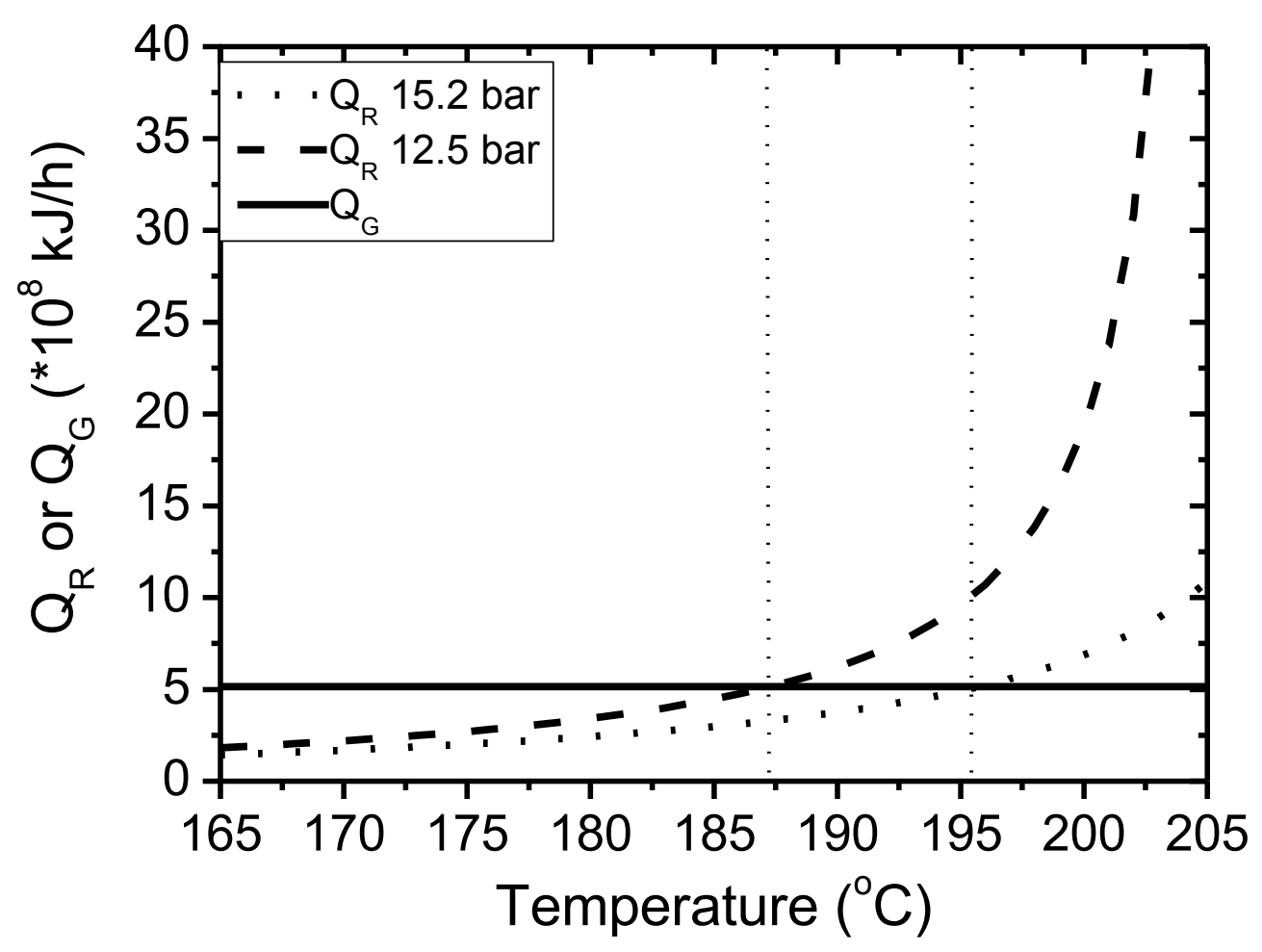

Figure 3.4: Predicted heat generation $\left(Q_{\mathrm{G}}\right)$ and heat removal $\left(Q_{\mathrm{R}}\right)$ rates for $p \mathrm{X}$ oxidation reactor (conditions noted in Figure 3.3)

\section{Effect of water content in feed on reactor temperature}

Modeling of the stirred reactor at MC process conditions clearly shows that a substantial portion of the heat generated by the oxidation reactions is removed by the evaporation of the solvent. The modeling results also reveal that proper control of the reactor operating pressure is essential to limit the reactor temperature to the boiling point of the solvent and thereby prevent temperature overshoot.

The feed stream in the MC process typically contains about $5 \mathrm{wt} \%$ water. Since water has a lower boiling point and a higher value of heat of vaporization compared to acetic acid, water content in the solvent affects the steady state reactor temperature at a given operating pressure. 
To quantify this effect, the mathematical model developed previously is applied to the MC oxidation reactor conditions at various water contents in the feed. Figure 3.5 shows the results of such an analysis. Higher water content in the solvent results in lower reactor temperature.

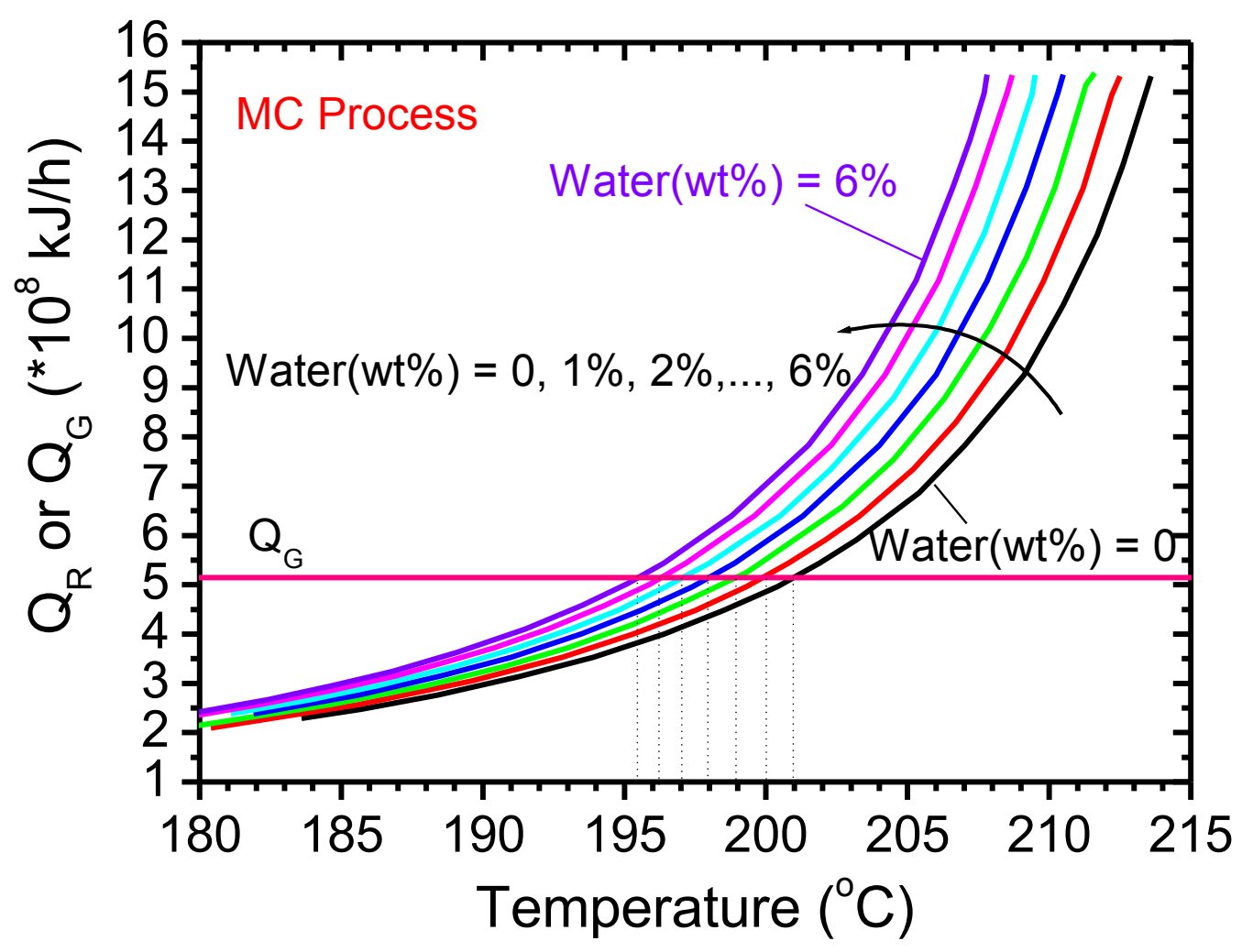

Figure 3.5: Effects of water content on reactor temperature in MC process

\subsubsection{Vapor phase flammability considerations}

In both the MC process and the spray process, the use of a flammable organic solvent (acetic acid) during $p \mathrm{X}$ oxidation gives rise to vapor phase flammability concerns. The upper flammability limit (UFL) for acetic acid in air under ambient or elevated pressure (above 30 bar) is below $25 \mathrm{vol} \%[6,82]$. In the spray reactor, an $\mathrm{O}_{2} / \mathrm{CO}_{2}$ mixture (instead of air) is used as the oxidant source with the maximum $\mathrm{O}_{2}$ content being $50 \mathrm{vol} \%$. Carbon dioxide is used as the 
diluent for $\mathrm{O}_{2}$ because of its higher heat capacity and superior flame inhibition properties relative to nitrogen. Under typical experimental $\mathrm{P}$ and $\mathrm{T}\left(15\right.$ bar and $\left.200{ }^{\circ} \mathrm{C}\right)$, the estimated vapor phase acetic acid and $\mathrm{O}_{2}$ concentrations at equilibrium are $58 \mathrm{vol} \%$ and $23 \mathrm{vol} \%$ respectively, assuming no reaction. The remaining component in the vapor phase is $\mathrm{CO}_{2}(\sim 19$ vol\%). The vapor phase component compositions were obtained from HYSYS simulation of a separator under the selected $\mathrm{P}$ and $\mathrm{T}$. For estimating vapor-liquid equilibrium, the UNIQUAC model was applied and Peng-Robinson equation of state was used to model the vapor phase [115]. $\mathrm{CO}_{2} /$ acetic acid binary interaction coefficient was assumed to be 0.02 [116]. Because $\mathrm{O}_{2}$ is fed at nearstoichiometric amounts needed for $p \mathrm{X} \rightarrow$ TPA oxidation, typical vapor phase $\mathrm{O}_{2}$ partial pressure is about 0.4 bar [98] or approximately 3 vol\% in the MC oxidation reactor. Using Calculated Adiabatic Reaction Temperature (CART) approach, Rajagopalan [6] demonstrated that even a vapor phase containing acetic acid at a molar concentration of $20 \%$ and equimolar amounts of $\mathrm{CO}_{2}$ and $\mathrm{O}_{2}$ (i.e., $40 \mathrm{~mol} \%$ each) falls outside the flammability envelope. Thus, the presence of $\mathrm{CO}_{2}$ as an inert gas and excess acetic acid $(>50 \mathrm{~mol} \%)$ in the vapor phase under reaction conditions create an environment that falls outside of the flammability envelope.

\subsection{Experimental Demonstration}

\subsubsection{Materials}

The substrate $(p \mathrm{X})$, catalyst materials (cobalt acetate, manganese acetate and $48 \%$ hydrobromic acid in water), solvent (acetic acid) and internal standard (biphenyl) were purchased from Sigma-Aldrich and used without further treatment. Ultra high purity (UHP) grade oxygen was purchased from Airgas. Industrial grade $\mathrm{CO}_{2}$ and equimolar $\mathrm{CO}_{2} / \mathrm{UHP} \mathrm{O}_{2}$ gas mixtures were purchased from Linweld. 


\subsubsection{Oxidation experiments in spray reactor}

Figure 3.6 shows the schematic of a $700 \mathrm{~mL}$ titanium spray reactor equipped with a 4-point thermocouple and a transducer for measuring axial reactor temperatures and the reactor pressure, respectively. Four profile thermocouples are located inside the reactor, with intervals of 1.5 inches. The temperature and pressure are continuously recorded and controlled with the help of a LabVIEW $^{\circledR}$ data acquisition system. The reactor lid accommodates multiple inlet/outlet ports for (a) liquid injection via a custom-made titanium nozzle (BETE ${ }^{\circledR}$, Model PJ), (b) continuously introducing an $\mathrm{O}_{2} / \mathrm{CO}_{2}$ gas mixture to the gas sparger immersed in the liquid phase at the bottom of the spray tower, (c) continuously withdrawing the vapor phase, and (d) a safety pressure relief valve. 


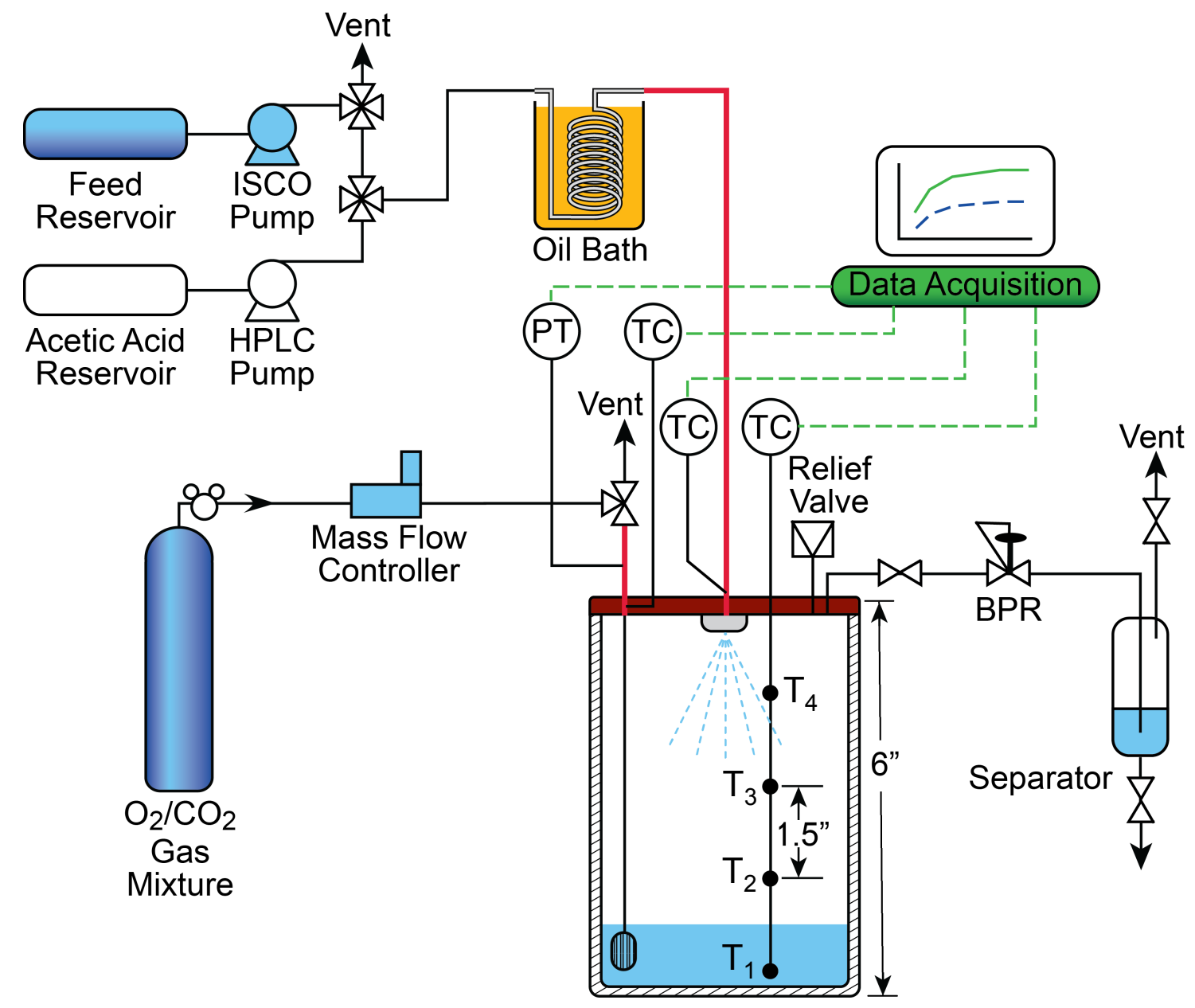

Figure 3.6: Schematic of the spray reactor: The four thermocouple locations (black dots) are evenly spaced and numbered from the bottom to the top as follows: $T_{1, \text { bottom }}$ (in the liquid phase),

$$
\mathrm{T}_{2} \text { (above } \mathrm{T}_{1, \text { bottom}),} \mathrm{T}_{3} \text { (above } \mathrm{T}_{2} \text { ) and } \mathrm{T}_{4, \text { top }}\left(\text { above } \mathrm{T}_{3}\right. \text { ). }
$$

The spray reactor was operated in a semi-batch mode with and without gas withdrawal. In a typical experimental run, the reactor was initially charged with $50 \mathrm{~mL}$ of acetic acid $(97 \mathrm{wt} \%$ purity, used as received) and pressurized with an equimolar $\mathrm{O}_{2} / \mathrm{CO}_{2}$ gas mixture, and then heated to the desired temperature (approximately $200^{\circ} \mathrm{C}$ ) such that the eventual pressure is 15 bar. After the temperature and pressure stabilized, the $\mathrm{O}_{2} / \mathrm{CO}_{2}$ gas mixture was preheated to the reactor temperature by means of an oil bath and continuously introduced into the reactor at a flow rate of $606 \mathrm{std} \mathrm{cm}^{3} / \mathrm{min}$. Simultaneously, the pre-mixed and pre-heated feed solution (containing 
specific concentrations of $p \mathrm{X}$, cobalt acetate, manganese acetate and hydrobromic acid dissolved in acetic acid) was sprayed into the reactor for 3 minutes at a rate of $35 \mathrm{~mL} / \mathrm{min}$. For a typical run, the $\mathrm{O}_{2} / p \mathrm{X}$ (mol:mol) is approximately 3.26 which is sufficient to fully convert the $p \mathrm{X}$ to TPA. In the constant pressure operation mode, the gas was also continuously withdrawn from the vapor phase to maintain the reactor pressure constant at the desired value (15 bar) with a backpressure regulator (DRESSER ${ }^{\circledR}$, Model SD91LW) connected to the gas-effluent line. In the experiments without gas withdrawal, the pressure was allowed to rise continuously during the 3minute duration of the spray. In both cases, the liquid volume increased by approximately 105 $\mathrm{mL}$ during the 3-minute spray duration. At the end of a run, the spray as well as the $\mathrm{O}_{2}$ addition was stopped and the reactor was allowed to cool down with any further reaction occurring mostly in the liquid phase in the presence of residual $\mathrm{O}_{2}$. Both the spray and the stirred reactors were typically allowed to cool down to room temperature in a programmed fashion ensuring that any observed differences in product quality in the two reactors are not due to temperature differences during the cooling step.

\subsubsection{Benchmark oxidation experiments in a stirred reactor}

To enable comparisons with the spray reactor performance, experiments were also performed in a $50 \mathrm{~mL}$ titanium stirred vessel (Parr Instrument Company, Series 4560 Mini Bench Top Reactor). Details of this reactor unit and operation are provided elsewhere [3]. Briefly, $25 \mathrm{~mL}$ of acetic acid ( $\sim 97 \mathrm{wt} \%$ in water) solution containing the catalyst components, whose concentrations were identical to those in the spray reactor experiments, was initially charged into the Parr reactor. The reactor was then pressurized with an equimolar $\mathrm{O}_{2} / \mathrm{CO}_{2}$ mixture to the desired operating pressure $(15 \mathrm{bar})$ and temperature $\left(200^{\circ} \mathrm{C}\right)$. The stirred reactor was operated in a semi-batch mode in which $p \mathrm{X}$ was continuously pumped into the reactor at a rate of 0.067 
$\mathrm{mL} / \mathrm{min}$ for 3 minutes. The molar $\mathrm{O}_{2} / p \mathrm{X}$ ratio based on the $p \mathrm{X}$ added and the $\mathrm{O}_{2}$ partial pressure of 3.2 bar is 2.5 . To ensure that there was always sufficient stoichiometric $\mathrm{O}_{2}$ supply to complete the $p \mathrm{X}$ oxidation to TPA, the $\mathrm{O}_{2}$ consumed during reaction is continuously replenished from an external $\mathrm{O}_{2}$ reservoir to maintain a constant partial pressure of 3.2 bar. There is about $58 \mathrm{~mol} \%$ acetic acid vapor in the gas phase. The total 15 bar pressure consists of $58 \%$ acetic acid and equimolar $\mathrm{O}_{2}$ and $\mathrm{CO}_{2}$ in the gas phase. The $\mathrm{O}_{2}$ pressure profile in the external reservoir was continuously monitored to maintain the desired rate of $\mathrm{O}_{2}$ consumption and total $\mathrm{O}_{2}$ consumption.

\subsubsection{Product analysis}

The solid product was filtered, washed, dried and analyzed by HPLC using the gradient elution technique described by Viola and Cao [102]. The sample preparation procedure and the chromatographic column used in this work are identical to those used in the work by Zuo et al. [3]. Specifically, the mobile phase consisted of three eluents: aqueous phase $(0.1 \mathrm{wt} \%$ phosphoric acid) and organic phase (consisting of 7 parts acetonitrile and 2 parts methanol by volume). The gradient elution program was as follows: starting with an aqueous phase, the eluent mixture composition was changed linearly during a five minute span to $95 \mathrm{vol} \%$ aqueous phase and 5 vol\% organic phase. From 5 to $10 \mathrm{~min}$, the mixture composition was altered linearly with time to $15 \mathrm{vol} \%$ aqueous phase and $85 \mathrm{vol} \%$ organic phase and kept constant thereafter until 16 min. The total flow rate of the mobile phase was $1 \mathrm{~mL} / \mathrm{min}$. A UV detector was used to quantify the products using biphenyl as internal standard $(215 \mathrm{~nm}$ for $p \mathrm{X}$ and $254 \mathrm{~nm}$ for all other products).

The 4-CBA detection limit is $200 \mathrm{ppm}$ for the aforementioned HPLC method. In order to increase the 4-CBA detection sensitivity to less than $25 \mathrm{ppm}$ in the TPA solid (and thereby 
enable assessment of polymer-grade purity), the following sensitive HPLC method was developed: a $20 \mathrm{mg}$ solid sample was dissolved in $20 \mathrm{~mL}$ of water with $150 \mu \mathrm{L}$ ammonium hydroxide and analyzed by HPLC. The gradient elution program is the same as explained above except that during the time period of 10-16 min, a mobile phase composition of $60 \mathrm{vol} \%$ aqueous phase and 40 vol\% organic phase was used with a total flow rate of $1 \mathrm{~mL} / \mathrm{min}$. The detection limit of this sensitive analytical method is down to $1 \mathrm{ppm} 4-\mathrm{CBA}$. The overall carbon balance based on analysis of $p \mathrm{X}$ and the oxidation products (in the liquid and solid phases) is around $95 \%$ in all experiments.

\subsection{Experimental results and discussion}

\subsubsection{Roles of liquid sparger}

A high surface area 4-blade titanium sparger immersed into the liquid phase for introducing the $\mathrm{O}_{2} / \mathrm{CO}_{2}$ gas mixture into the liquid (Figure 3.7) was attempted to maintain acetic acid saturation and sufficient $\mathrm{O}_{2}$ availability in the vapor and liquid phases. To test the liquid sparger in a blank run, the amounts of acetic acid condensed from the reactor overhead stream (vapor phase) were measured. As shown in Figure 3.8, without sparger, the acetic acid flow rate decayed with time and the vapor phase tended to dry out (undesired). Further, temperature gradients were observed in the reactor. With sparger, however, acetic acid collected from overhead condenser fluctuated around $1.4 \mathrm{~g} / \mathrm{min}$ and temperature gradients were minimized. 

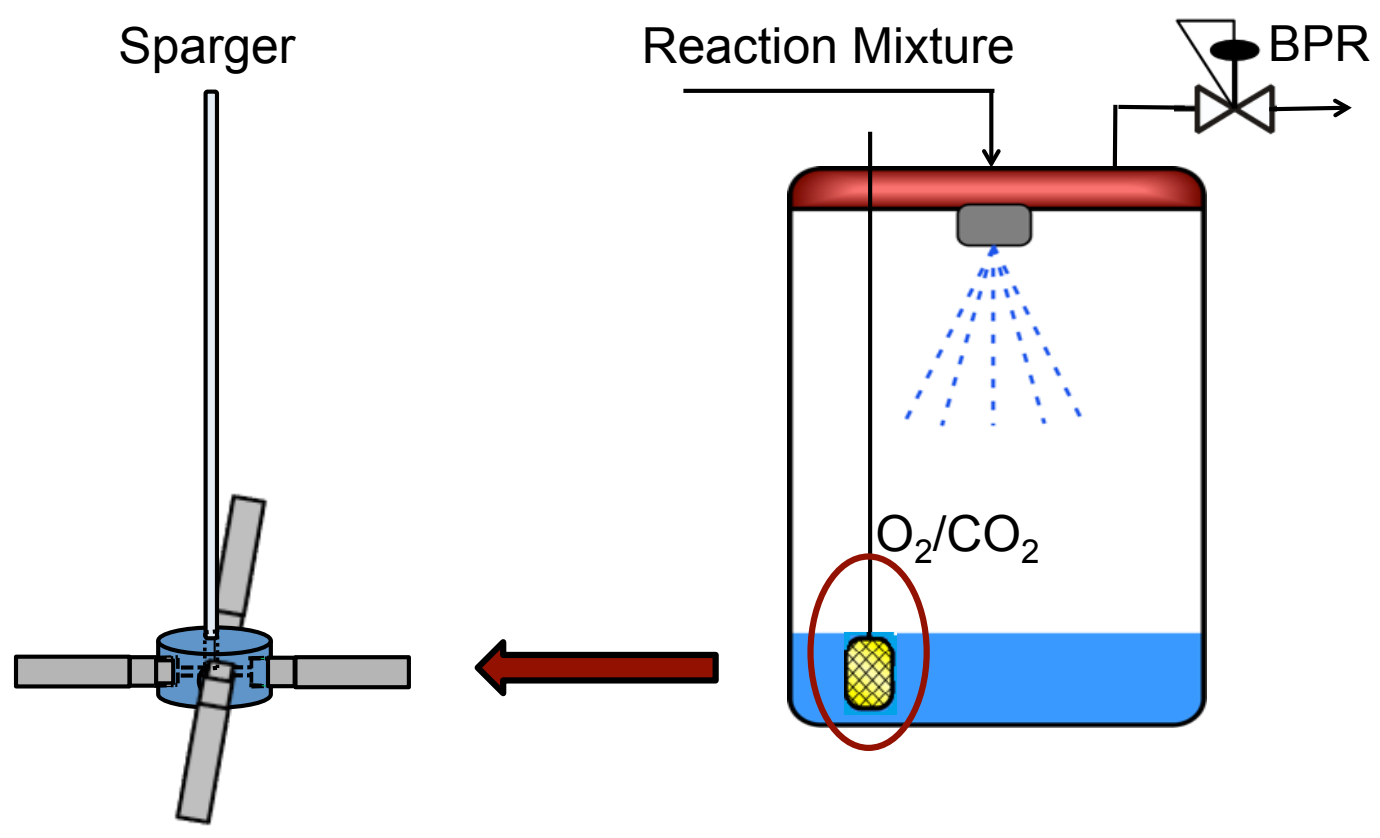

Figure 3.7: Schematic of the liquid sparger
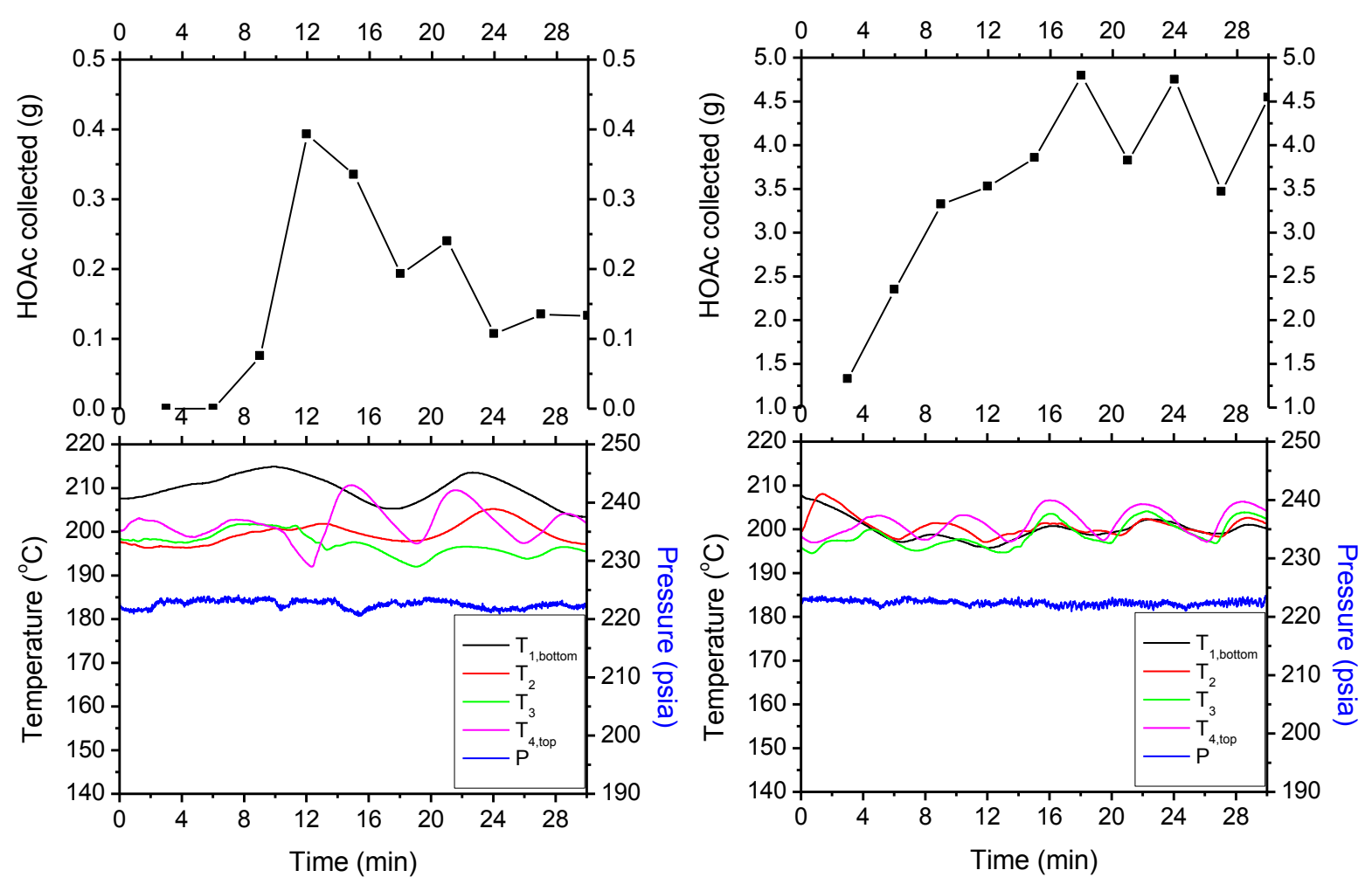

Figure 3.8: Measurement of acetic acid condensed from gas phase during blank run

Left: without sparger; Right: with sparger 


\subsubsection{Performance of spray reactor}

Table 3.2 shows a summary of the solid TPA product purities from the spray reactor. The experimental procedure was described in section 3.2.2. In addition to the main product and intermediates shown in Figure 1.3, the presence of 4-(bromomethyl)benzoic acid (BPTA) was also checked during product analysis. It was found that the spray reactor produced high-purity solid TPA product with 4-CBA at concentrations of less than $25 \mathrm{ppm}$ (Table 3.2) being the only impurity detected. The TPA crystals produced from the spray reactor were white and needle-like with particles in the $600-1500 \mu \mathrm{m}$ size range, as seen from SEM (scanning electron microscope) image (Figure 3.9). A UV-Vis spectrometer was used to determine the optical density of the solid TPA product as an indicator of the extent of colored compounds present. The measurement of optical density and details of associated calculations are provided elsewhere [3]. The typical $\mathrm{OD}_{340}$ (optical density at $340 \mathrm{~nm}$ ) values of the obtained solid TPA product from the spray reactor are in the range of $0.013-0.018$. These values meet the specification required for a polymer-grade TPA product $(<0.08)[6]$.

Table 3.2: Purity of solid TPA product obtained from the spray reactor

\begin{tabular}{|c|c|c|c|c|c|c|c|}
\hline \multirow[b]{2}{*}{ Run \# } & \multirow[b]{2}{*}{ Operation mode } & \multirow{2}{*}{$\begin{array}{c}\mathrm{T} \\
\left({ }^{\circ} \mathrm{C}\right)\end{array}$} & \multirow{2}{*}{$\begin{array}{c}\mathrm{P} \\
\text { (bar) }\end{array}$} & \multirow{2}{*}{$\begin{array}{l}p \mathrm{X} \text { added } \\
(\mathrm{mmol})\end{array}$} & \multicolumn{3}{|c|}{ Solid product quality } \\
\hline & & & & & $\begin{array}{l}\text { 4-CBA } \\
(\mathrm{ppm})\end{array}$ & $\begin{array}{l}\text { BPTA } \\
(\mathrm{ppm})\end{array}$ & $\begin{array}{l}p \text {-TA } \\
(\mathrm{ppm})\end{array}$ \\
\hline 1 & $\begin{array}{l}\text { With gas outlet } \\
\text { (P constant) }\end{array}$ & 200 & 15 & 13.8 & $3 \pm 1$ & n.d. ${ }^{a}$ & n.d. \\
\hline $2^{b}$ & $\begin{array}{l}\text { With gas outlet } \\
\text { (P constant) }\end{array}$ & 200 & 15 & 20.7 & $<1$ & n.d. & n.d. \\
\hline $3^{c}$ & $\begin{array}{l}\text { With gas outlet } \\
\text { ( } P \text { constant })\end{array}$ & 200 & 15 & 27.6 & $<1$ & n.d. & n.d. \\
\hline 4 & $\begin{array}{l}\text { No gas outlet } \\
\text { (P increases) }\end{array}$ & $200-235$ & $30-38$ & 27.5 & $13 \pm 1$ & n.d. & n.d. \\
\hline
\end{tabular}




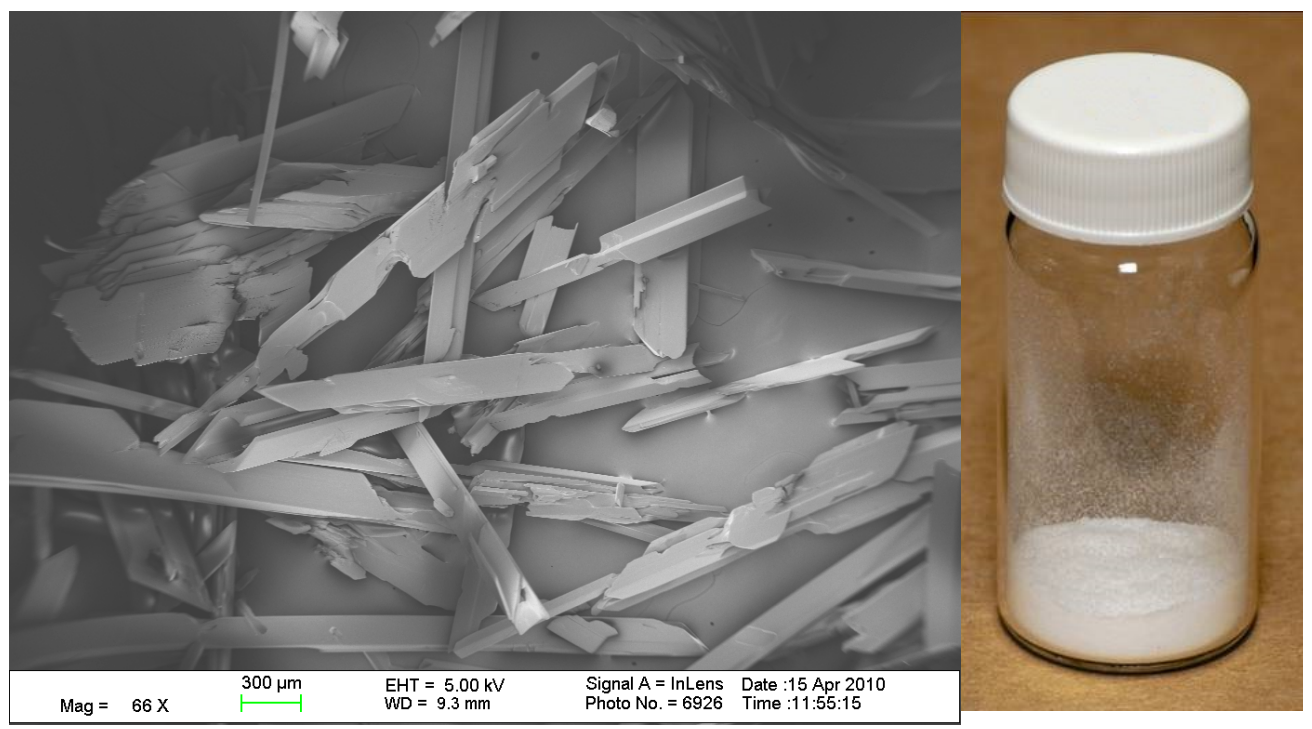

Figure 3.9: SEM (scanning electron microscope) image of TPA crystals produced from the spray reactor

Figures $3.10 \mathrm{a}$ and $3.10 \mathrm{~b}$ show the temperature and pressure profiles corresponding to runs 1 and 4 (Table 3.2). During operation without gas outlet (Figure 3.10b), the reactor pressure increased by up to approximately 7.5 bar (from 30 to 38 bar) and the temperature increased by up to $35^{\circ} \mathrm{C}$ with the maximum temperature occurring in the liquid phase at the bottom of the spray reactor. In sharp contrast, when the reactor pressure was maintained at a constant value during reactor operation with gas outlet (Figure 3.10a), the temperature profiles were nearly flat and the temperature rise was within a few degrees. The significant temperature rise in Figure $3.10 \mathrm{~b}$ is partly attributed to the greater amount of heat generated at the higher $p \mathrm{X}$ concentrations and partly to the increasing pressure (30-38 bar) during the run that increases the boiling point of the solvent and lowers the evaporative cooling effect. When an experiment using the same $p \mathrm{X}$ concentration as that in run 4 was performed at 15 bar with gas outlet (run 3), similar T \& P profiles as in run 1 (Figure 3.10c) were obtained. This indicates that the increasing reactor 
pressure in run 4 was the main reason for the increasing $\mathrm{T}$ during that run. The increase in $\mathrm{T}$ enhances the possibility of not only solvent burning but also titanium which partially burns in pure $\mathrm{O}_{2}$ at $200{ }^{\circ} \mathrm{C}$ and $\mathrm{O}_{2}$ partial pressure of roughly 25 bar $[6,117]$. Clearly, a reactor pressure of $15 \mathrm{bar}$ is preferred to take better advantage of evaporative cooling due to acetic acid boiling at lower $\mathrm{T}\left(232.5^{\circ} \mathrm{C}\right.$ at 15 bar, Figure 3.2), as explained in section 3.1.2.

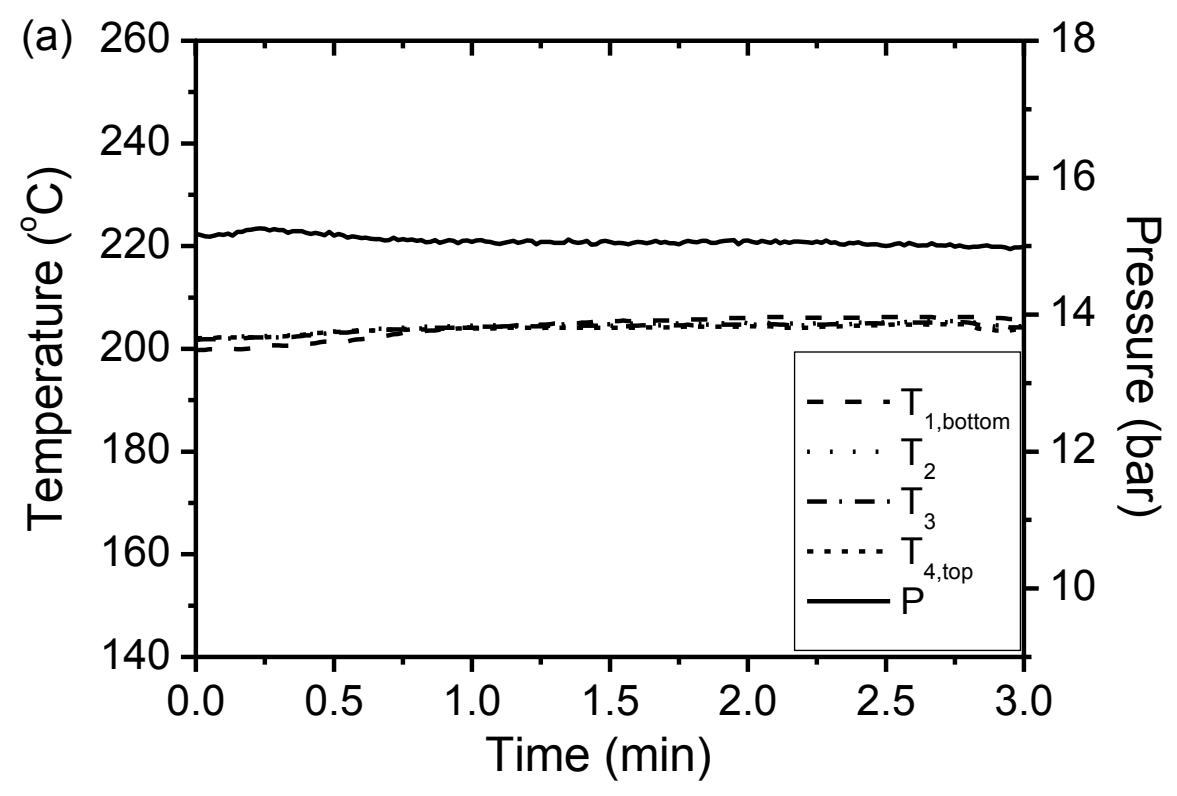



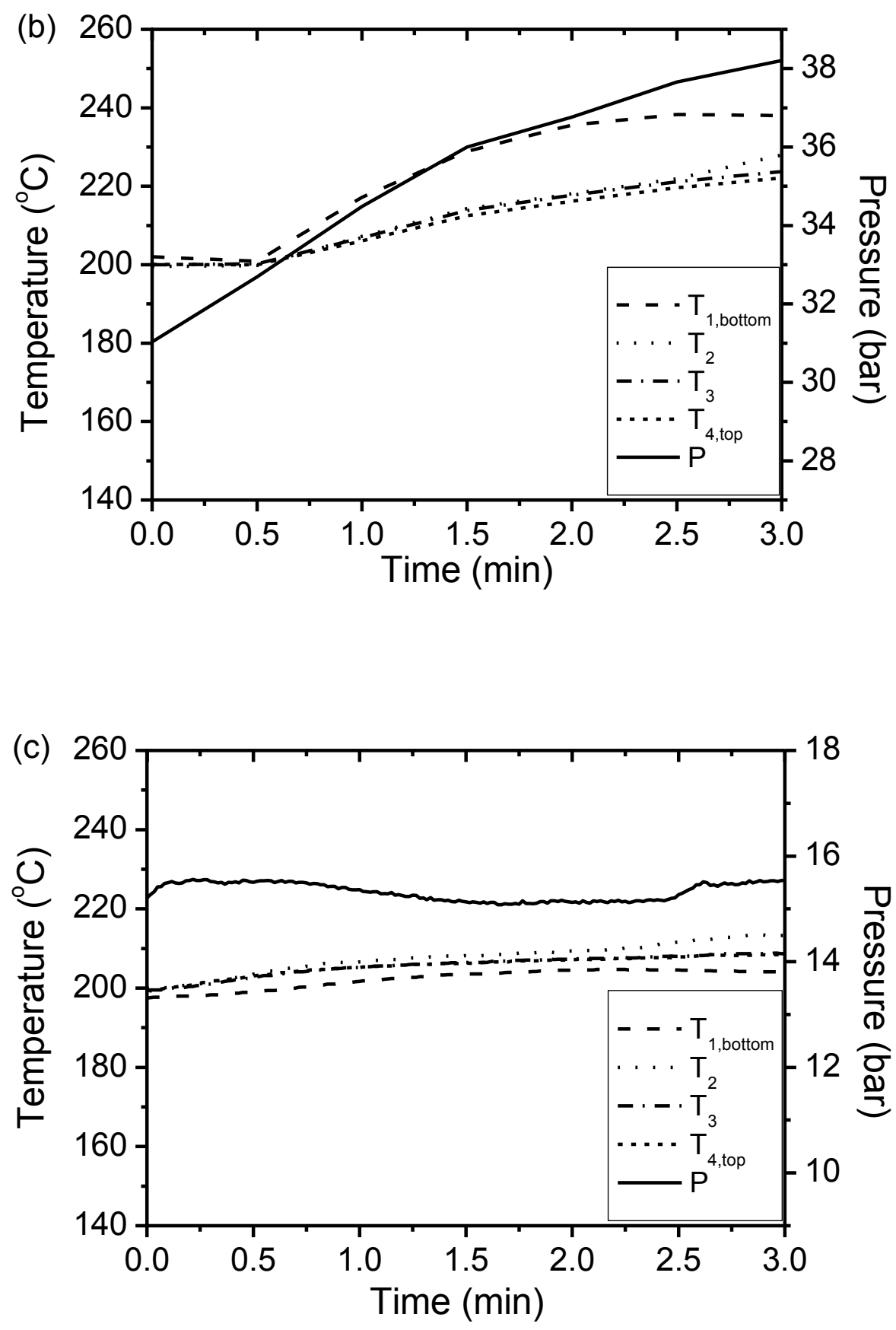

Figure 3.10: Temporal temperature and pressure profiles during spray reactor operation:
(a) with gas outlet (operating conditions correspond to run 1, Table 3.2);
(b) without gas outlet (operating conditions correspond to run 4, Table 3.2);
(c) with gas outlet (operating conditions correspond to run 3, Table 3.2). 
It is important to point out that the reaction solution was preheated to the desired temperature via a two-stage oil bath before being sprayed into the reactor. During the development phase of the spray reactor, a single stage oil bath was used resulting in an inlet temperature of $<150{ }^{\circ} \mathrm{C}$ just before the liquid stream passed through the nozzle. Even though the entire reactor was heated to $200{ }^{\circ} \mathrm{C}$ and other reaction conditions stayed the same as those aforementioned experiments, the solid TPA produced under low inlet temperatures was yellowish and the 4-CBA content was on the order of tens of thousands of ppm, clearly confirming the importance of preheating the reaction mixture to $200{ }^{\circ} \mathrm{C}$ before contacting with $\mathrm{O}_{2}$ to avoid the kinetic limitations at the lower temperatures and take full advantage of the increased $\mathrm{O}_{2} /$ liquid mass transfer area.

Table 3.3 summarizes the results of analyses of the solid and liquid phases from run \#1 (Table 3.2). The majority of the TPA product exists in the solid phase. The high-purity solid TPA product harvested following a 3-minute spray is approximately $2.07 \mathrm{~g}$, translating to an overall productivity of $394 \mathrm{~kg} \mathrm{~m}^{-3} \mathrm{~h}^{-1}$ based on the liquid phase volume. While only minor amounts of 4CBA and TALD intermediates were detected in the liquid, a significant amount of BPTA was also found. It was found that this intermediate can be completely eliminated (i.e., oxidized to TPA) if the reactor temperature was maintained at $200{ }^{\circ} \mathrm{C}$ for 30 minutes following the spray. Further, $p$-TA, a major impurity in industrial grade purified TPA, was not detected in either the solid or liquid phases. In this study, only the concentrations of the aromatic compounds were used to calculate carbon balance, which was found to be around $95 \%$, which is within experimental error. 
Table 3.3: Product specifications from run \#1 of Table 3.2

\begin{tabular}{cccc}
\hline Item & Solid phase & Liquid phase & $\begin{array}{c}\text { Overall yield } \\
\text { (solid + liquid phases) }\end{array}$ \\
\hline TPA & $99.99+\%$ & $289 \pm 10 \mathrm{ppm}$ & - \\
TPA yield & $91.3 \%$ & $2.7 \%$ & $94.0 \%$ \\
4-CBA & $3 \pm 1 \mathrm{ppm}$ & $<2 \mathrm{ppm}$ & $0.0087 \%$ \\
BPTA & n.d. & $58 \pm 10 \mathrm{ppm}$ & $0.42 \%$ \\
$p$-TA & n.d. & n.d. & n.d. \\
TALD & n.d. & $<2 \mathrm{ppm}$ & $0.024 \%$ \\
${ }^{\text {aCCarbon balance }}$ & - & - & $94.5 \%$ \\
\hline
\end{tabular}

${ }^{a}$ Based on analysis of $p \mathrm{X}$ fed and oxidation products in the liquid and solid phases

\subsubsection{Comparison of product from spray and stirred reactors}

Table 3.4 compares the TPA yield and solid product quality from the spray reactor and the stirred reactor with identical initial $p \mathrm{X}$ concentrations and under similar reactor pressures and temperatures. While the spray reactor facilitates near-complete $p \mathrm{X}$ oxidation producing nearly pure TPA product (4-CBA $<25 \mathrm{ppm}$ ), the solid TPA produced from the stirred reactor contains 1000-4000 ppm 4-CBA, which falls in the range of crude TPA from the conventional MC process. Further, the presence of the $p$-TA and BPTA in the solid TPA product from the stirred reactor implies oxygen starvation in the liquid phase [39]. At all conditions investigated, the spray reactor produces similar TPA yield as a stirred reactor with a carbon balance closure of above $94 \%$ for each experiment but significantly better solid TPA product that conforms to polymer-grade purity. 
Table 3.4: Comparison of product purity and yields from spray and stirred reactors

\begin{tabular}{|c|c|c|c|c|c|c|c|c|c|c|c|c|}
\hline \multirow{2}{*}{$\begin{array}{l}\text { Run } \\
\#\end{array}$} & \multirow{2}{*}{ Reactor } & \multirow{2}{*}{\multicolumn{2}{|c|}{$\begin{array}{cc}\mathrm{T} & \mathrm{P} \\
\left({ }^{\circ} \mathrm{C}\right)(\mathrm{bar})\end{array}$}} & \multirow{2}{*}{$\begin{array}{c}\text { Initial } \\
p \mathrm{X} \\
\text { conc. } \\
(\mathrm{M})\end{array}$} & \multicolumn{3}{|c|}{$\begin{array}{l}\text { Impurities in solid TPA } \\
\text { product }\end{array}$} & \multicolumn{5}{|c|}{$\begin{array}{c}\text { Overall yield (\%) } \\
\text { (solid + liquid phases) }\end{array}$} \\
\hline & & & & & $\begin{array}{l}\text { 4-CBA } \\
\text { (ppm) }\end{array}$ & $\begin{array}{l}p \text {-TA } \\
(\mathrm{ppm})\end{array}$ & & & $p$-TA & BPTA & 4-CBA & TPA \\
\hline 4 & & $\begin{array}{l}200- \\
235\end{array}$ & $\begin{array}{l}30- \\
37\end{array}$ & 0.264 & $13 \pm 1$ & n.d. & n.d. & & 0.46 & 0.64 & 0.10 & 97.6 \\
\hline 5 & Stir & 200 & 30 & 0.264 & $1,621 \pm 10$ & 1,062 & n.d. & & 1.57 & 0.96 & 0.61 & 96.0 \\
\hline 6 & Spray & 200 & 15 & 0.066 & $46 \pm 1$ & n.d. & n.d. & 0.015 & - & 0.072 & 0.0044 & 94.8 \\
\hline 7 & Stirred & 200 & 15 & 0.066 & $924 \pm 10$ & n.d. & $986 \pm 10$ & 0.21 & 0.68 & 5.23 & 0.36 & 92.7 \\
\hline 8 & & 200 & 15 & 0.066 & $<1$ & n.d. & n.d. & - & - & - & - & 95.6 \\
\hline 9 & Stirred $^{\mathrm{a}}$ & 200 & 15 & 0.066 & $112 \pm 1$ & n.d. & n.d. & 0.11 & 0.27 & - & 0.07 & 93.7 \\
\hline
\end{tabular}

Reaction conditions:

$\mathrm{Co}=12.5 \mathrm{mM}, \mathrm{Mn}=12.5 \mathrm{mM}, \mathrm{Br}=32.5 \mathrm{mM}$;

$\mathrm{O}_{2}: \mathrm{CO}_{2}(\mathrm{~mol}: \mathrm{mol})=1: 1$

spray rate $=35 \mathrm{~mL} / \mathrm{min}$, spray time $=3 \mathrm{~min}$;

gas mixture flow rate $\left(\mathrm{O}_{2}: \mathrm{CO}_{2}=1: 1\right)=606 \mathrm{std} \mathrm{cm}^{3} / \mathrm{min}$;

${ }^{a}$ Reactors were maintained at $200{ }^{\circ} \mathrm{C}$ for 1 hour after completion of $p \mathrm{X}$ introduction.

Typically, the products were removed from the reactor following a cool-down phase after a run. It was found that the stirred reactor cooled down faster than the spray reactor. While the stirred reactor cooled from $200{ }^{\circ} \mathrm{C}$ to approximately $160{ }^{\circ} \mathrm{C}$ in 10 minutes (Figure $3.11 \mathrm{a}$ ), the temperature of the liquid phase in the spray reactor was nearly $190{ }^{\circ} \mathrm{C}$ after 10 minutes of natural cooling (Figure 3.11b). To rule out the effects of higher temperatures during the cool-down phase in promoting more complete oxidation of the intermediates, experiments were conducted wherein the stirred reactor temperature was maintained at $200{ }^{\circ} \mathrm{C}$ for $1 \mathrm{~h}$ following completion of $p \mathrm{X}$ introduction. In this manner, the average temperatures during the cool-down phase were much higher in the stirred reactor compared to the spray reactor. Despite the higher temperatures, the 4-CBA content in the TPA product from the stirred reactor was still $112 \mathrm{ppm}$, several-fold 
greater than the 4-CBA concentrations attained with the spray reactor. In fact, if the spray reactor is also maintained at $200{ }^{\circ} \mathrm{C}$ for $1 \mathrm{~h}$ following cessation of $p \mathrm{X}$ introduction, the 4-CBA content in the solid TPA product almost vanishes. This shows that the reaction also occurs in the liquid phase. Overall, the spray reactor is able to provide much better oxidation rates of $p \mathrm{X}$ and of the intermediates compared to the stirred reactor, resulting in a much better TPA product quality (i.e., with lower concentrations of intermediates). This finding has important practical implications as it shows that with proper optimization, a continuous spray reactor has the potential to produce polymer-grade TPA obviating the need for the hydrogenation reactor used in the conventional MC process. The resulting savings in capital and operating costs, and in GHG emissions (due to reduction in hydrogen use and solvent burning) can be significant and lead to improved process sustainability. These potential benefits are quantified and discussed in Chapter 5.

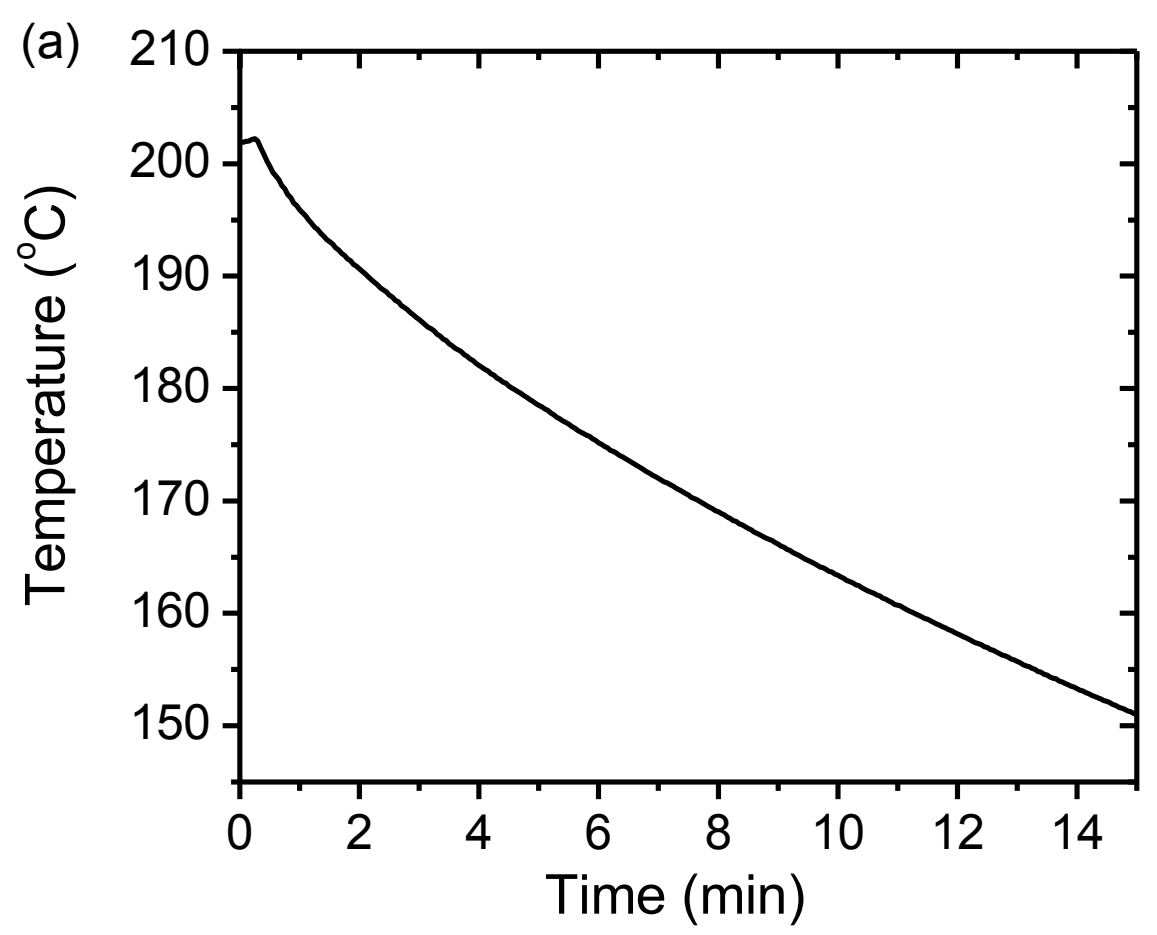




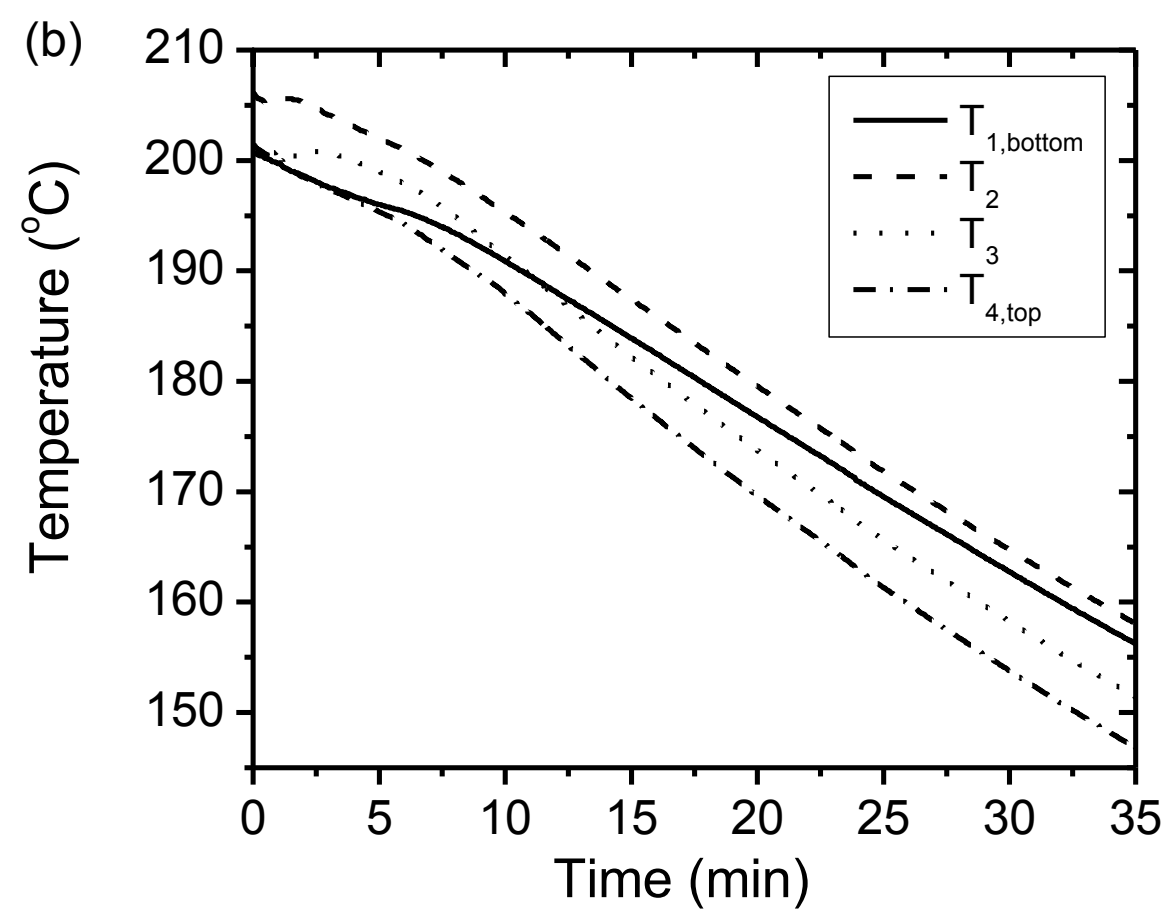

Figure 3.11: Temperature profiles during the cool down phase (a) stirred reactor; (b) spray reactor.

\subsection{4 $\mathrm{CO}$ formation as a measure of burning reactions}

The increase in the concentration of $\mathrm{CO}_{2}$ in the gas phase is typically measured to assess the extent of burning reactions during $p \mathrm{X}$ oxidation. Because we use a $\mathrm{CO}_{2} / \mathrm{O}_{2}$ mixture, it is difficult to accurately measure incremental $\mathrm{CO}_{2}$ concentrations in the gas phase due to burning reactions. Hence, we chose to measure the $\mathrm{CO}$ concentration in the gas phase as an approximate measure of the extent of burning reactions. The gas mixture was analyzed by GC (column: Shin Carbon ST 100/120 mesh) to determine the CO formation from solvent burning. Preliminary experimental results from the spray process (Table 3.5) show that at identical feed conditions, the observed $\mathrm{CO}$ formation during spray reactor operation was approximately one fourth or less of that observed during stirred reactor operation $[\mathrm{CO} / \mathrm{pX}(\mathrm{mol} / \mathrm{mol}) \sim 0.045]$. The reduced burning is 
presumably because the residence time in the spray reactor $(\sim 1.5 \mathrm{~min})$ is much shorter than that in the MC reactor $(\sim 35 \mathrm{~min})$. Further experiments encompassing longer spray durations and ${ }^{14} \mathrm{CO}_{2}$-labeling studies are needed to confirm the extent of solvent burning during spray reactor operation.

Table 3.5: $\mathrm{CO}$ analysis during the $p \mathrm{X}$ oxidation in the spray reactor

\begin{tabular}{ccc}
\hline Run \# & Time duration for vapor sample collection & $\mathrm{CO} / \mathrm{pX}(\mathrm{mol} / \mathrm{mol})$ \\
\hline 10 & $1^{\prime} 25^{\prime \prime} \sim 2^{\prime} 45^{\prime}$ & 0.0112 \\
11 & $40^{\prime \prime} \sim 2^{\prime} 15^{\prime}$ & 0.0048 \\
12 & $1^{\prime} \sim 3^{\prime} 05^{\prime}$ & 0.0114 \\
\hline
\end{tabular}

Reaction conditions:

$\mathrm{T}=200^{\circ} \mathrm{C}, \mathrm{P}=15$ bar;

$\mathrm{Co}=12.5 \mathrm{mM}, \mathrm{Mn}=12.5 \mathrm{mM}, \mathrm{Br}=32.5 \mathrm{mM}$;

$p \mathrm{X}$ added $=6.9 \mathrm{mmol}$, spray rate $=35 \mathrm{~mL} / \mathrm{min}$.

\subsection{Summary}

For $p$-xylene oxidation to TPA, a spray reactor in which the liquid phase is dispersed as fine droplets into the gas phase containing oxygen produces high-purity TPA $(<25 \mathrm{ppm} 4-\mathrm{CBA})$ in one step at $200{ }^{\circ} \mathrm{C}$ and 15 bar pressure. This result is in sharp contrast to the performance of the conventional stirred reactor in which the solid TPA product contains $>1000 \mathrm{ppm}$ 4-CBA requiring further purification steps. We attribute the superior performance of the spray reactor to increased $\mathrm{O}_{2}$-liquid mass transfer area thus avoiding starvation that can occur when gas is sparged into a continuous liquid phase, as in the stirred reactor. In addition, the reduced backmixing in the spray reactor has also contributed to enhanced conversion. Another advantage of the spray reactor is that the solvent (acetic acid) burning rate is reduced due to the shorter residence times in the spray reactor, which was demonstrated by our preliminary experimental 
results of $\mathrm{CO}$ formation rate. Theoretical calculations confirm that in typical spray droplets, the $\mathrm{O}_{2}$ diffusion time constants are one to two orders of magnitude lower than the kinetic time constant (associated with the rate determining step) implying facile penetration of the $\mathrm{O}_{2}$ throughout the liquid phase and elimination of $\mathrm{O}_{2}$ starvation. Further, mathematical modeling of a stirred reactor operated at typical Mid-Century process conditions shows that solvent evaporation is a main source of cooling that results in steady states that are open-loop stable. The modeling also shows that control of reactor operating pressure around 15 bar is critical to achieving stable operation without temperature overshoot that might lead to solvent and titanium burning in the presence of oxygen. These model predictions were verified experimentally. Spray reactor operation in which the reactor pressure is maintained constant results in constant pressure and temperatures whereas operation in which the reactor pressure is allowed to rise during continuous gas introduction results in a steadily rising temperature in the reactor. In addition, higher water content in the solvent results in lower reactor temperature in both the MC and spray processes because water has a lower boiling point and a higher value of heat of vaporization compared to acetic acid. Further, the presence of $\mathrm{CO}_{2}$ and acetic acid vapor in the gas phase in sufficiently high concentrations is shown to maintain the reaction outside the flammability envelope. The elimination of the hydrogenation step and reduction of solvent burning (due to smaller residence times compared to the stirred reactor) provide potential economic and environmental benefits that would make a continuous spray process a greener alternative to the conventional Mid-Century process. Furthermore, this work also suggests that the spray process may be extended to other fast gas-liquid reactions to overcome gas-liquid mass transfer limitations in conventional stirred reactors. 


\section{Chapter 4 Development of a Continuous Spray Process for Terephthalic Acid Production}

In the previous chapter, a semi-batch spray process concept for the oxidation of $p \mathrm{X}$ to TPA was successfully demonstrated. This chapter is intended to demonstrate the spray process concept in continuous operation mode. Some preliminary experimental results from the continuous spray process are reported.

\subsection{Continuous Spray Reactor: Initial Efforts}

A major goal of this research program is to develop and demonstrate a continuous, laboratoryscale spray reactor process in which polymer-grade $(<25$ ppm $4-\mathrm{CBA})$ TPA is produced as a solid product in one step at high yields (comparable to the Mid-Century process) while simultaneously reducing the solvent (acetic acid) burn rate. In Chapter 3, it was demonstrated that a $700 \mathrm{~mL}$ spray reactor operated in semi-batch mode (i.e., gas phase is continuously introduced in and withdrawn from the spray reactor while liquid phase remains in the reactor during the 3-min spray duration) produces high-purity TPA ( $<25 \mathrm{ppm} 4-\mathrm{CBA})$. The next challenge to address is whether the high-purity product can be continuously withdrawn from the reactor in order to demonstrate a continuous process. The progress to date is described in this section.

The intrinsic kinetic studies of the homogeneous reaction system indicate that $p \mathrm{X}$ oxidation to TPA requires several minutes of reaction time in the liquid phase to convert the partial oxidation products to TPA. Given that the minimum spray rate of $35 \mathrm{~mL} / \mathrm{min}$ required by the nozzle to 
generate micron sized droplets, the $700 \mathrm{~mL}$ spray reactor with $50 \mathrm{~mL}$ of liquid phase (initially) would provide only 1-3 minutes of residence time (based on the liquid phase holdup) during continuous operation. In order to provide flexibility in allowing longer residence times, it was decided to construct a longer ( $2 \mathrm{ft}$.) $4 \mathrm{~L}$ spray reactor. In addition to allowing larger liquid holdups (and therefore longer residence times), the larger vessel also increases the zone in which the oxidations occur without backmixing, which is particularly important for achieving purity levels of $99.99 \%$. The dimensions of the two generations of spray reactors are shown in Figure 4.1.
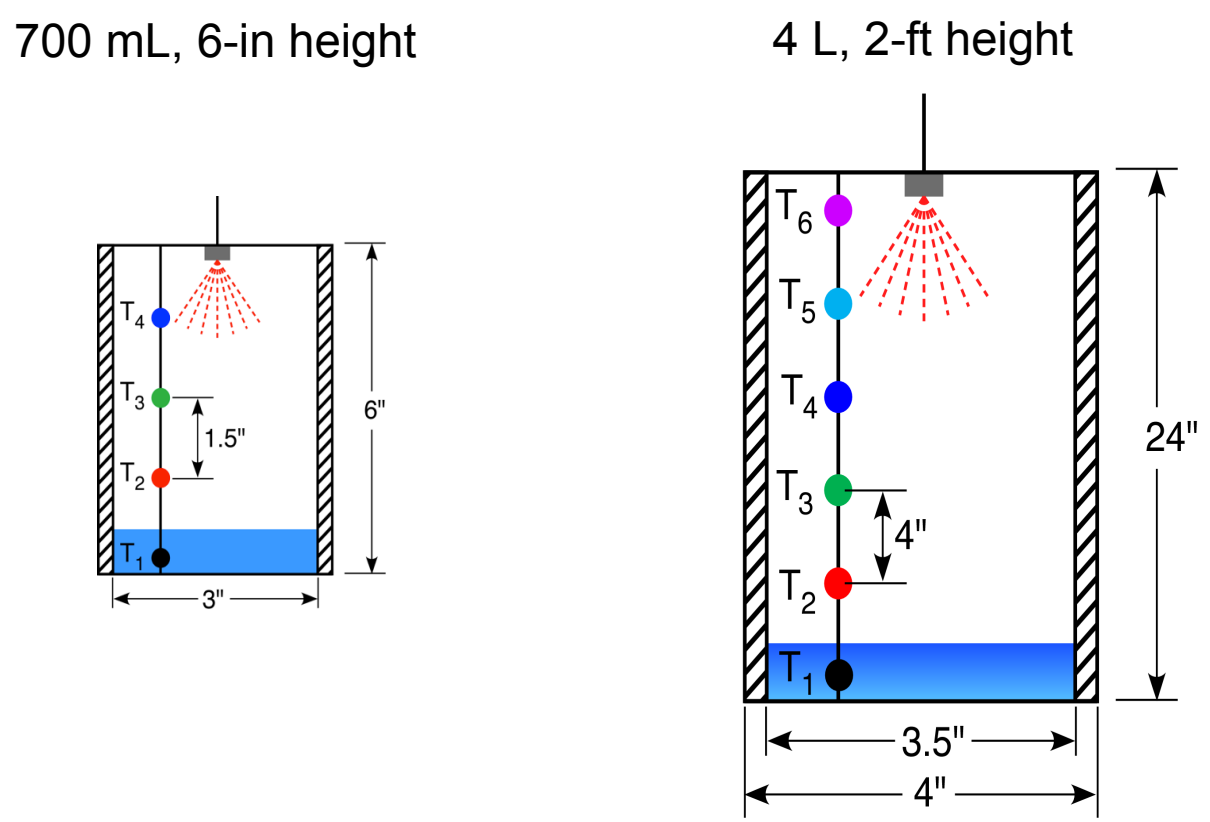

Figure 4.1: Dimensions of $700 \mathrm{~mL}$ and $4 \mathrm{~L}$ spray reactors

Table 4.1 shows the solid TPA product quality obtained from the $4 \mathrm{~L}$ spray reactor operated in semi-batch mode. The reaction conditions were identical to those employed in the $700 \mathrm{~mL}$ reactor (Table 3.3) except that $300 \mathrm{~mL}$ of acetic acid (instead of $50 \mathrm{~mL}$ ) were loaded in the $4 \mathrm{~L}$ reactor. The 4-CBA content in the solid TPA product was $93 \mathrm{ppm}$ and significant amounts of BPTA (449 ppm) were detected (run \#2). The higher impurity levels observed in the $4 \mathrm{~L}$ reactor 
compared to the $700 \mathrm{~mL}$ reactor were probably due to the faster cool-down resulting in unequal post-oxidation times in the two reactors. Indeed, polymer-grade TPA product was obtained given 15-min post-oxidation time at $200{ }^{\circ} \mathrm{C}$ (run \#4). Based on these results, we can conclude that high purity TPA is also obtainable in the longer $4 \mathrm{~L}$ reactor.

The experimental results shown in Table 4.1 also demonstrated the significant role of sparger by which $\mathrm{O}_{2} / \mathrm{CO}_{2}$ gas mixture was introduced into the liquid phase. Without sparger, polymergrade TPA product could not be obtained even given 1-hour post-oxidation time at $200{ }^{\circ} \mathrm{C}$ (run \#3). With sparger, however, the impurity contents of 4-CBA and BPTA are greatly reduced (run \#2). Further, polymer-grade TPA product is obtained given 15 -min post-oxidation time at $200{ }^{\circ} \mathrm{C}$ (run \#4). All this evidence shows that the vapor and liquid phases are better saturated with acetic acid and $\mathrm{O}_{2}$ with the help of the liquid sparger.

Table 4.1: Gas sparger effects on solid TPA product quality from the 4-L spray reactor

\begin{tabular}{cccccc}
\hline \multirow{2}{*}{$\begin{array}{c}\text { Run } \\
\#\end{array}$} & Sparger & TPA purity (wt\%) & 4-CBA (ppm) & BPTA (ppm) & $\begin{array}{c}p \text {-TA } \\
(\mathrm{ppm})\end{array}$ \\
\cline { 3 - 6 } & & $99.59 \pm 0.01$ & $1689 \pm 1$ & $2367 \pm 1$ & n.d. $^{\text {a }}$ \\
\hline 1 & No & $99.95 \pm 0.01$ & $93 \pm 1$ & $449 \pm 1$ & n.d. \\
2 & Yes & $99.97 \pm 0.01$ & $278 \pm 1$ & n.d. & n.d. \\
$3^{\text {b }}$ & No & $99.99+$ & $19 \pm 1$ & n.d. & n.d. \\
$4^{\text {c }}$ & Yes & & & & \\
\hline
\end{tabular}

Reaction conditions:

$\mathrm{T}=200{ }^{\circ} \mathrm{C} ; \mathrm{P}=15$ bar; $p \mathrm{X}$ added $=6.9 \mathrm{mmol} ; \mathrm{Co}=12.5 \mathrm{mM}, \mathrm{Mn}=12.5 \mathrm{mM}, \mathrm{Br}=32.5 \mathrm{mM}$; spray time $=3 \mathrm{~min}$; spray rate $=35 \mathrm{~mL} / \mathrm{min} ; \mathrm{O}_{2}: \mathrm{CO}_{2}(\mathrm{~mol}: \mathrm{mol})=1: 1$; gas mixture flow rate $=$ $600 \mathrm{scc} / \mathrm{min}$

an.d.: not detected;

${ }^{b}$ Reactor was maintained at $200^{\circ} \mathrm{C}$ for 1 hour after spray;

${ }^{\mathrm{c}}$ Reactor was maintained at $200{ }^{\circ} \mathrm{C}$ for 15 min after spray. 


\subsection{Preliminary Experimental Results from Continuous operation of the 4 L Spray Reactor}

The performance of the sparged continuous spray reactor was investigated initially using a manually controlled micro-metering valve in the liquid effluent stream. The experimental setup is similar to the semi-batch spray reactor setup (Figure 3.6) with the only difference being that there is an outlet at the bottom of the reactor and a manually controlled micro-metering valve is used to control the liquid effluent flow rate. In a typical experimental run, the reactor was initially pressurized with an equimolar $\mathrm{O}_{2} / \mathrm{CO}_{2}$ gas mixture, and then heated to the desired temperature (approximately $200{ }^{\circ} \mathrm{C}$ ). After the temperature stabilized, the $\mathrm{O}_{2} / \mathrm{CO}_{2}$ gas mixture was preheated to the reactor temperature by means of heating tape and continuously introduced into the reactor at a flow rate of $600 \mathrm{std} \mathrm{cm}^{3} / \mathrm{min}$. Simultaneously, the gas was also continuously withdrawn on the top of the reactor from the vapor phase to maintain the reactor pressure constant at the desired value $\left(15\right.$ bar) with a back-pressure regulator (DRESSER ${ }^{\circledR}$, Model SD91LW) connected to the gas-effluent line. Approximately $300 \mathrm{~mL}$ of acetic acid was first sprayed into the reactor (approximately 8 minutes and 40 seconds with a flow rate of 35 $\mathrm{mL} / \mathrm{min}$ ). Then the pre-mixed and pre-heated feed solution (containing specific concentrations of $p \mathrm{X}$, cobalt acetate, manganese acetate and hydrobromic acid dissolved in acetic acid; $[p \mathrm{X}]=66$ $\mathrm{mM} ;[\mathrm{Co}]=12.5 \mathrm{mM},[\mathrm{Mn}]=12.5 \mathrm{mM},[\mathrm{Br}]=32.5 \mathrm{mM}$;) was sprayed into the reactor at ate of $35 \mathrm{~mL} / \mathrm{min}$, while simultaneously opening the micro-metering valve to withdraw the liquid phase from the bottom of the reactor. For a typical run, the $\mathrm{O}_{2} / p \mathrm{X}$ (mol:mol) is approximately 5.8 which is sufficient to fully convert the $p \mathrm{X}$ to TPA. The initial $p \mathrm{X}$ concentration $(0.066 \mathrm{M})$ is low enough to maintain the produced TPA completely dissolved in the liquid phase. 
Excellent $\mathrm{T} \& \mathrm{P}$ control and constant acetic acid flow rate from the bottom of the reactor were obtained during a 25-min blank run. T \& P profiles and collected acetic acid amounts (from the bottom liquid phase and the gas stream exiting at the top of the reactor) are shown in Figure 4.2 and Table 4.2. HYSYS simulation (Figure B1 in Appendix B) gave a flow rate of the overhead acetic acid vapor stream of approximately $1.8 \mathrm{~g} / \mathrm{min}(\sim 9 \mathrm{~g}$ for $5 \mathrm{~min}$ duration) in a continuous reactor with vapor phase reaching equilibrium. The condensed acetic acid from overhead, obtained from the blank experiment, is shown to be 7.3-8.1 g during 5 minutes. Considering the possible acetic acid loss from the overhead condenser, it is surmised that the vapor phase is most probably saturated with acetic acid. This needs to be verified more thoroughly in future work.

Table 4.2: Acetic acid amounts from the top (gas stream) and bottom (liquid stream) of the spray reactor (operating conditions same as in Table 4.1)

\begin{tabular}{ccc}
\hline Time duration $(\mathrm{min})$ & $\begin{array}{c}\text { Condensed HOAc from } \\
\text { overhead }(\mathrm{g})\end{array}$ & HOAc from bottom $(\mathrm{g})$ \\
\hline $0-5$ & 8.01 & 207 \\
$5-10$ & 6.30 & 206 \\
$10-15$ & 8.08 & 205 \\
$15-20$ & 7.40 & 203 \\
$20-25$ & 7.36 & 204 \\
\hline
\end{tabular}




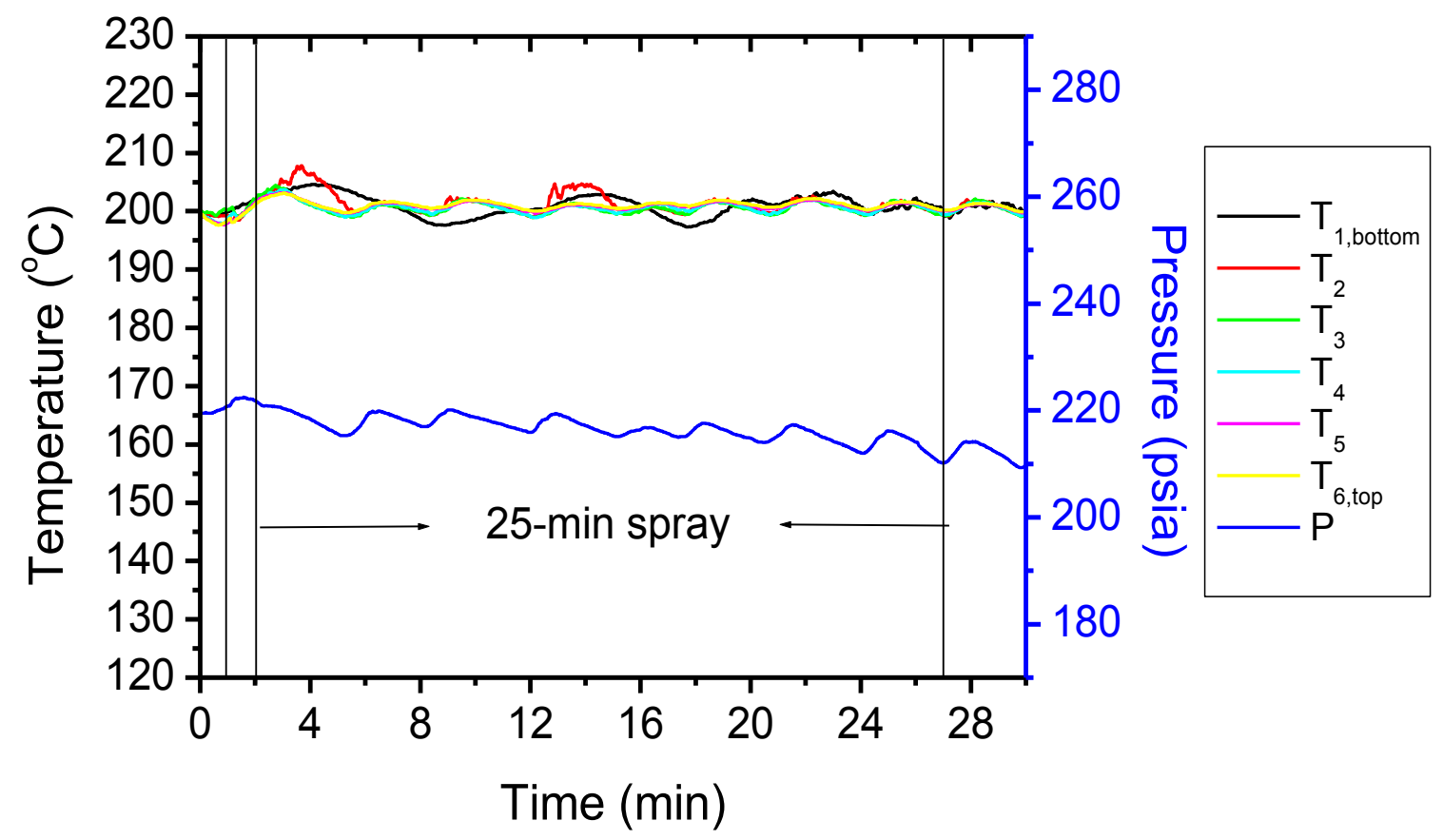

Figure 4.2: T \& P profiles during a 25-min blank run with manual control of micro-metering valve

However, in the preliminary reaction run, manual control led to varying liquid holdup in the reactor with time as shown in Table 4.3, as well as high concentrations of intermediates probably due to low catalyst concentration and/or inadequate residence time in the liquid phase. To get better liquid level control (and therefore more constant residence time in the liquid phase), automated liquid level control of the liquid level was implemented. A stepper-motor-controlled micro-metering valve for liquid level control was designed by Mr. Edwin Atchison (Electronics Technician, CEBC), employing the instantaneous reactor mass as feedback. Constant $\mathrm{T} \& \mathrm{P}$ profiles and acetic acid weight in the reactor were obtained during a 30-min blank run (Figure 4.3). This should permit further testing and development of the continuous spray reactor by future researchers. 
Table 4.3: Solid TPA product quality during 25 -min continuous run in $4 \mathrm{~L}$ spray reactor

\begin{tabular}{cccccc}
\hline \multirow{2}{*}{$\begin{array}{c}\text { Time duration } \\
(\mathrm{min})\end{array}$} & \multirow{2}{*}{$\begin{array}{c}\text { Liquid Holdup } \\
(\mathrm{mL})\end{array}$} & $\begin{array}{c}\text { TPA purity } \\
(\mathrm{wt} \%)\end{array}$ & $\begin{array}{c}\text { 4-CBA } \\
(\mathrm{ppm})\end{array}$ & BPTA (ppm) & $p$-TA (ppm) \\
\hline $0-5$ & 125 & 98.15 & 5,423 & 13,065 & n.d. \\
$5-10$ & 127 & 98.87 & 3,294 & 8,012 & n.d. \\
$10-15$ & 116 & 99.08 & 1,821 & 7,339 & n.d. \\
$15-20$ & 176 & 99.03 & 1,489 & 8,217 & n.d. \\
$20-25$ & $\sim 300$ & 98.92 & 1,489 & 9,304 & n.d. \\
\hline
\end{tabular}

$p \mathrm{X}=0.066 \mathrm{M}$; other reaction conditions are the same as those for Table 4.1.
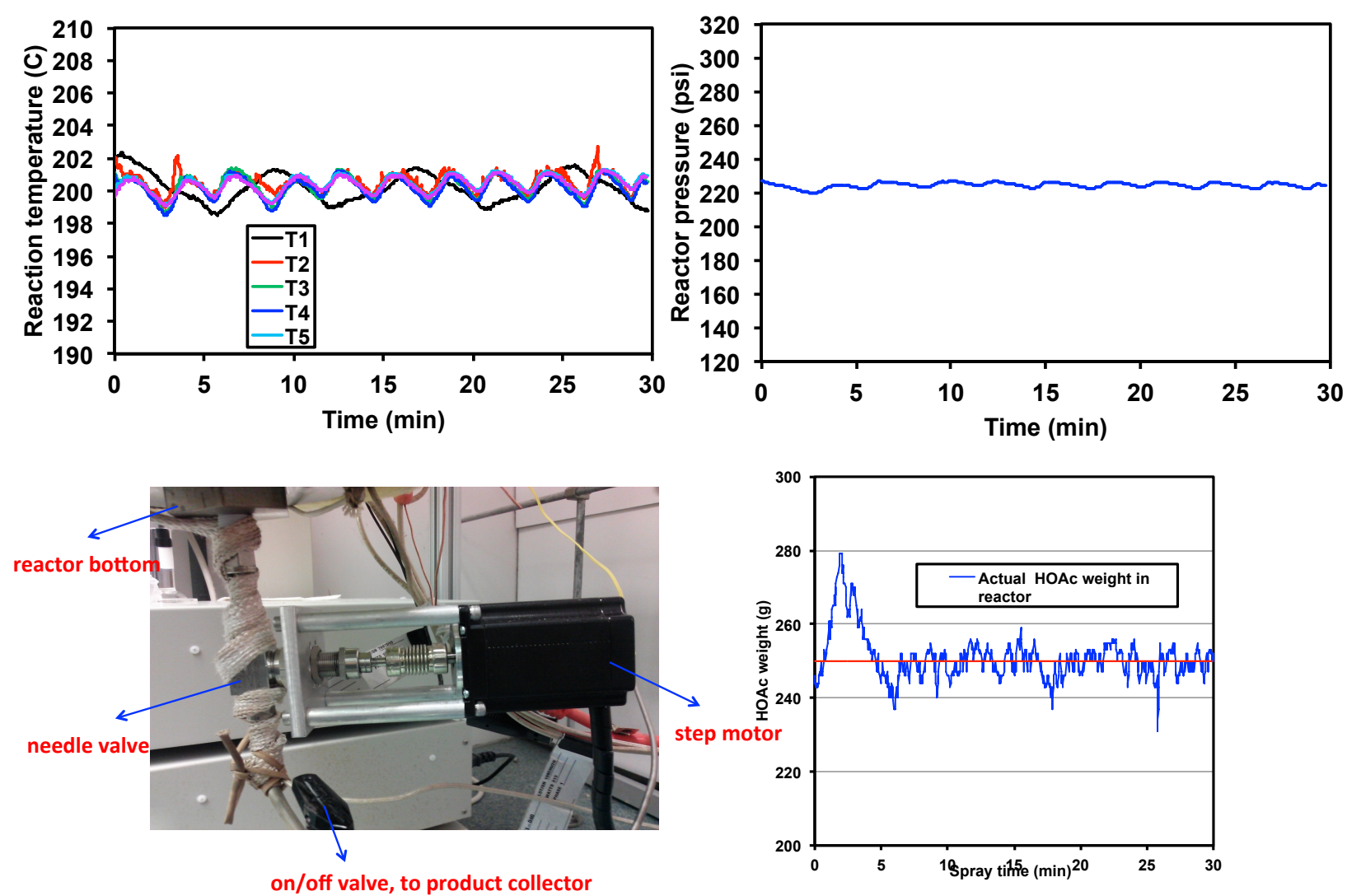

Figure 4.3: 30-min blank run with stepper motor controlled micro-metering valve 


\subsection{Summary}

Preliminary experimental results of $p \mathrm{X}$ oxidation from a $4 \mathrm{~L}$ continuous spray reactor during a 25-min experimental run using a manually controlled micro-metering valve show high concentrations of undesirable intermediates products, which could be due to several reasons such as non-steady state operation with varying liquid holdup in the reactor caused by the manual control, low catalyst concentration and inadequate residence time in the liquid phase. To get a steady state continuous operation, automatic control of the bottom liquid phase flow rate must be used. A stepper-motor-controlled micro-metering valve was successfully designed and implemented in the 4-L continuous spray reactor. Excellent $\mathrm{T} \& \mathrm{P}$ control as well as constant acetic acid weight in the reactor were obtained during a 30-min blank run. This should permit testing and development of the continuous spray reactor for $p \mathrm{X}$ oxidation by future researchers. 


\section{Chapter 5 Economic and Environmental Impact Assessment of the Spray Process for Terephthalic Acid Production}

With dwindling natural resources and increasing global population, sustainable development has assumed increased importance in this new millennium. The well-accepted definition of sustainability is "development that meets the needs of the present without compromising the ability of future generations to meet their own needs" [118]. Sustainability indicators include economic, environmental and social issues $[119,120]$. A sustainable process is one that covers all of these three dimensions.

As stated by the International Council of Chemical Associations (ICCA) in the United Nations Environmental Programme's GEO-2002, the chemical industry has laid a solid platform for moving toward becoming a sustainable sector. Many companies are continuing to evaluate resource-efficient manufacturing processes, and substituting more sustainable products as alternatives, where appropriate [121]. The CEBC mission is well aligned with this sustainability goal. The development of the CEBC spray process as a potentially more sustainable substitute for the conventional stirred process is exemplary of its efforts to promote sustainability in the chemical industry sector. The technical feasibility of the spray process concept has been demonstrated in Chapter 3. This chapter deals with quantitative comparison of the economics and potential environmental impacts of the CEBC spray process and the conventional MC process to assess the practical viability and the environmental benignity of the alternative spray process for terephthalic acid production. 


\subsection{Introduction}

It has been demonstrated in Chapter 3 that the CEBC spray process in which the liquid phase containing dissolved $p \mathrm{X}$ and the catalyst is dispersed as fine droplets into the gas phase containing oxygen produces high-purity TPA $(<25 \mathrm{ppm} 4-\mathrm{CBA})$ in one step at $200{ }^{\circ} \mathrm{C}$ and 15 bar pressure. The elimination of the hydrogenation step and the reduction of solvent burning provide economic and environmental benefits that make a continuous spray process as a potentially more sustainable alternative to the conventional Mid-Century process. The non-requirement of the energy-intensive hydrogenation section obviates a number of processing steps resulting in the reduction in energy requirements as well as capital and operating costs. The lower solvent burning rate reduces not only the cost for make-up acetic acid but also $\mathrm{CO}_{2}$ emissions. By conserving material and energy, and also reducing $\mathrm{CO}_{2}$ emissions, the spray process satisfies several principles of green chemistry [122]. However, quantitative sustainability assessment is essential to not only numerically determine the economic and environmental benefits but, equally importantly, to also identify areas for further improvement in sustainability-related metrics. Such a quantitative assessment is especially insightful when process conditions are different. For example, the $p \mathrm{X}$ concentration in the demonstrated spray process is 10 times lower compared to the industrial process. This means that the acetic acid that must be processed to meet a certain production requirement is ten times higher in the spray process. The processing of such large quantities of acetic acid increases not only equipment and energy costs, but also the $\mathrm{CO}_{2}$ emissions associated with solvent burning. These adverse effects could either partially or totally offset the potential economic and environmental benefits anticipated by eliminating the hydrogenation step. Clearly, quantitative comparative assessment of the economics and 
environmental impact assessments of the two processes are essential to unambiguously address and resolve such issues.

Several reported case studies of quantitative economic and environmental impact assessments of process design alternatives provide valuable guidance for the evaluation of this particular spray process. Dunn and Savage $[123,124]$ examined the economic feasibility and the environmental impact of a terephthalic acid process using high-temperature water (HTW) as the reaction medium. They found that a subcritical HTW-based process was equally capital intensive, less energy intensive, and more environmentally benign than the current acetic-acidbased process (MC process). Similar methodology of economic and environmental impact analyses have been employed to evaluate alternative chemical processes such as $\mathrm{CO}_{2}$-based hydroformylation process [125], solid-acid catalyzed alkylation process in supercritical $\mathrm{CO}_{2}$ [126], liquid-phase $\mathrm{H}_{2} \mathrm{O}_{2}$-based ethylene oxide process [127] and $\mathrm{H}_{2} \mathrm{O}_{2}$-based propylene oxide process [128], all of which were developed by CEBC researchers. The work presented herein is a relatively thorough quantitative evaluation of the CEBC spray technology for TPA production, from both economic and environmental points of view. The conventional MC process is employed as the industrial benchmark, against which the economics and environmental impacts of four different spray reactor case studies using varying amount of acetic acid in the feed are simulated and compared. The results reveal key operating factors that affect the economics and environmental impacts, and provide valuable guidance for process design and optimization.

\subsection{Methodology}

The methodology employed here generally includes three steps as follows. First, both the MC process and the CEBC spray process ( 4 cases with various acetic acid/p-xylene ratios in the feed) are simulated by the HYSYS ${ }^{\circledR}$ software assuming a certain TPA production capacity for the 
plant. Process flow diagrams (PFDs), developed for each technology with various operating parameters/variables and product specifications as inputs, provide the relevant mass and energy flow rates associated with the process streams. These values are used in process equipment design and sizing. Secondly, comparative economic analyses are performed by estimating capital investment and total production cost for each technology. The capital investment is estimated following the percentage of purchased-equipment cost method [129], in which the cost for purchased equipment is treated as the estimation basis. Other direct costs or indirect costs are estimated by multiplying the purchased equipment cost with a corresponding factor. The purchased equipment costs are estimated based on attributes such as equipment size, material of construction, weight or surface area $[129,130]$. For determining the production costs, the amounts of utilities and chemicals consumed are estimated from the HYSYS ${ }^{\circledR}$-estimated stream properties. The costs associated with utilities and raw materials labor are obtained from specified current sources. Finally, comparative gate-to-gate LCA (life cycle assessment) and cradle-to-gate LCA of both processes are performed to evaluate the relative environmental impacts $[127,128]$. The stream flows and compositions from the PFDs are used to estimate the potential environmental impacts associated with the emitted streams from both processes. More details of the methodologies are provided in the following sections.

\subsection{Process Simulations}

\subsubsection{Process simulation basis}

The design basis for both of the conventional MC process and the CEBC spray process is a production rate of 500,000 t/yr of purified terephthalic acid (PTA) at a 0.9 stream factor (i.e., 328 on-stream days). Plant-scale data for the simulated conventional MC process were obtained from published patent data $[27,28,132-134]$. Data for simulating the spray process were obtained 
from laboratory scale data reported in Chapter 3. Simulations of both processes were carried out with Aspen HYSYS ${ }^{\circledR}$ V7.3 software. For estimating vapor-liquid equilibrium, the UNIQUAC model was used to simulate the liquid phase composition and Peng-Robinson equation of state was used to model the vapor phase. $\mathrm{CO}_{2}$ /acetic acid binary interaction coefficient [116] was set as 0.02 , and other binary interaction coefficients are based on the HYSYS database. Tables 5.1 and 5.2 summarize the key operating parameters for process simulation basis and the assumptions, respectively, associated with acetic acid use and loss for the four cases of the CEBC spray process.

Table 5.1: Key operating parameters for the simulation of the MC and CEBC spray processes

\begin{tabular}{lcc}
\hline & MC Process & Spray Process \\
\hline Oxidation reactor conditions & & \\
Temperature $\left({ }^{\circ} \mathrm{C}\right)$ & 195 & 200 \\
Pressure (psia) & 220 & 220 \\
Water concentration in reaction solvent feed $(\mathrm{wt} \%)$ & 5 & 5 \\
$\quad p$-xylene conversion $(\%)$ & 98.1 & 99.6 \\
Solid product quality & & \\
$\quad$ 4-CBA concentration (ppmw) & 1375 & 23 \\
$\quad p$-TA concentration $(\mathrm{ppmw})$ & 608 & 2 \\
\hline Hydrogenation reactor conditions & & \\
Temperature $\left({ }^{\circ} \mathrm{C}\right)$ & 267 & - \\
Pressure (psia) & 975 & - \\
4-CBA conversion $(\%)$ & 90 & - \\
Solid product quality & & - \\
$\quad$ 4-CBA concentration (ppmw) & 7 & 97 \\
$\quad p$-TA concentration $(\mathrm{ppmw})$ & 100 & \\
\hline Overall TPA yield $(\%)$ & 95 & \\
\hline
\end{tabular}


Table 5.2: Assumed acetic acid use and loss for the MC process and the CEBC spray process

\begin{tabular}{cccccc}
\hline & \multirow{2}{*}{ MC process } & \multicolumn{4}{c}{ Spray process } \\
\cline { 3 - 6 } & & Case 1 & Case 2 & Case 3 & Case 4 \\
\hline HAc $/ p$ X feed ratio $(w t / w t)$ & 2.3 & 20.8 & 14.4 & 8.1 & 2.3 \\
HAc loss from oxidation reactor $(\%)$ & 5.3 & 1.3 & 1.3 & 1.3 & 1.3 \\
\hline
\end{tabular}

\subsubsection{Process descriptions}

The process flow diagrams of the conventional MC process and the CEBC spray process simulated by HYSYS ${ }^{\circledR}$ are shown in Figures $5.1 \&$ 5.2. Details of the simulation parameters, operating conditions of major unit operations and flow rates of key streams are provided in Tables C1 \& C2 of Appendix C. 


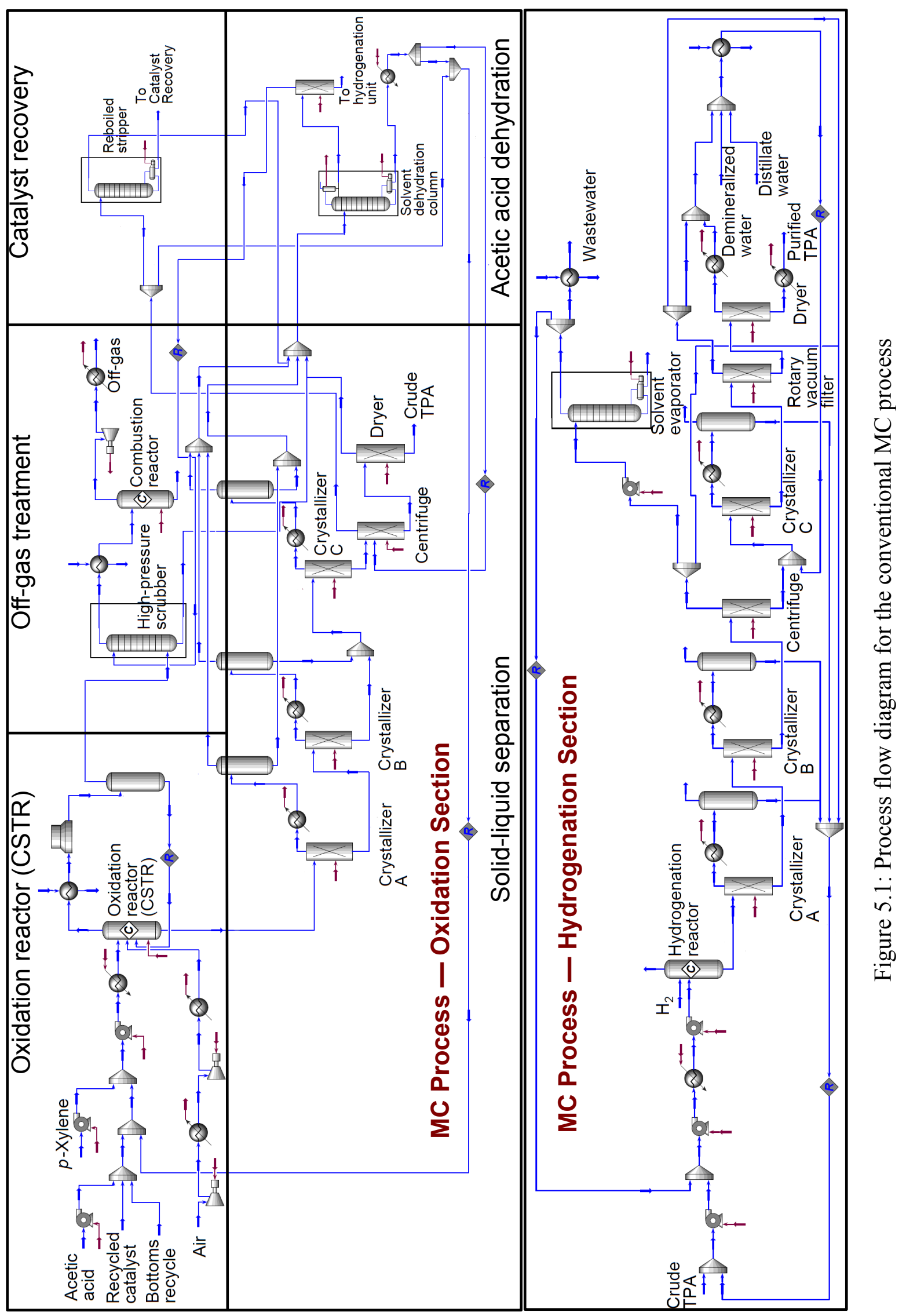




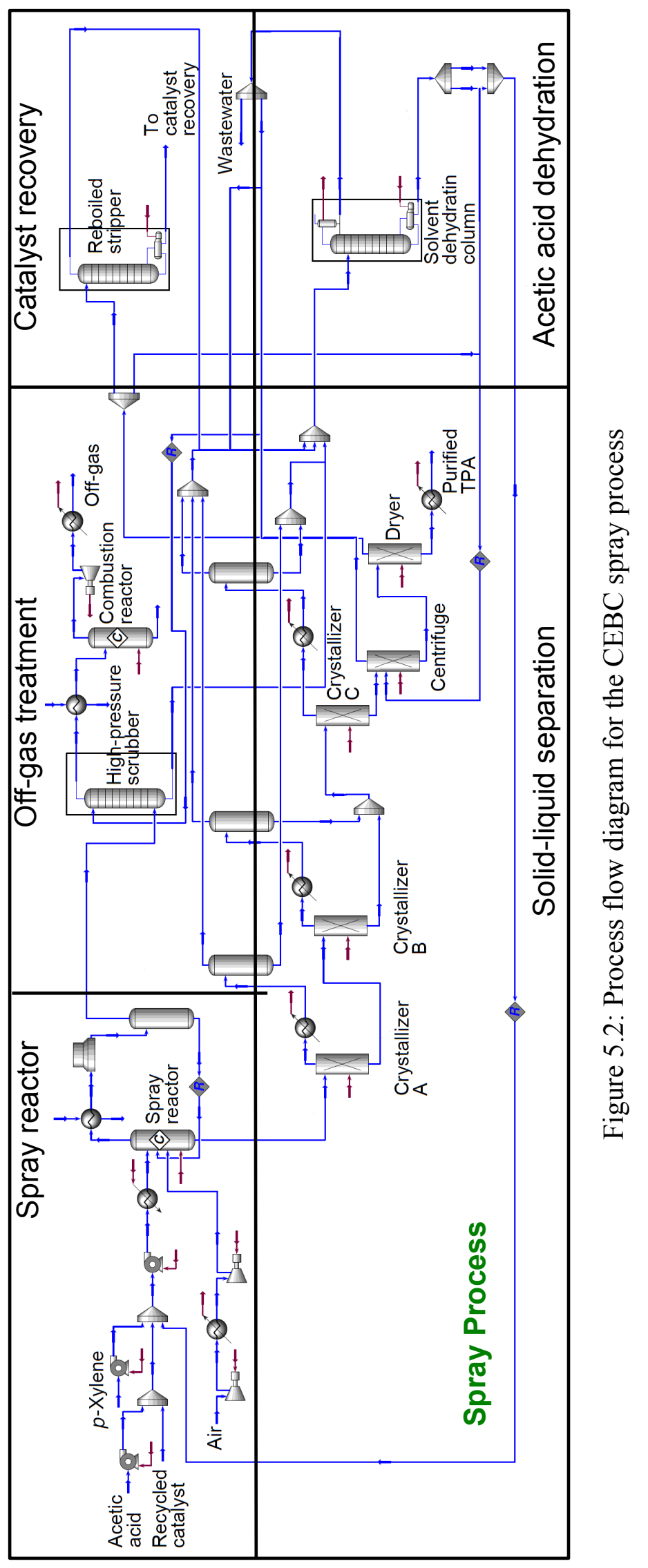




\subsubsection{Conventional MC process}

\section{Oxidation section}

\section{Oxidation reactor (CSTR)}

The feed solution pumped into the oxidation reactor contains $p$-xylene, catalyst (cobalt acetate, manganese acetate and hydrogen bromide) and solvent. Cobalt acetate and manganese acetate are mostly recovered in the catalyst recovery unit, so that hydrogen bromide is the only significant makeup component. Acetic acid is the solvent of choice in the TPA process primarily due to its unique interaction with Co to produce $\mathrm{Co}(\mathrm{III})$ that initiates the oxidation reaction [46]. In the MC process, the acetic acid solvent contains approximately $5 \mathrm{wt} \%$ of water [134]. The oxidation reactor requires a substantial amount of acetic acid, most of which is recycled via the solvent dehydration column where acetic acid is separated from water. The fresh (makeup) acetic acid accounts for approximately $3 \%$ of the amount of acetic acid fed to the oxidation reactor.

Air (oxidant) passes though two stages of compressors and is then also fed into the oxidation reactor. The oxidation reactor in the $\mathrm{MC}$ process is usually a stirred vessel in which $p \mathrm{X}$ is oxidized to TPA by the oxygen in the air. The reactor is assumed to be adiabatic and maintained at $200{ }^{\circ} \mathrm{C}$ and 15 bar under steady operation. A substantial amount of the heat generated from the highly exothermic reaction (the heat of reaction is $1,300 \mathrm{~kJ} / \mathrm{mol}$ at total $p \mathrm{X}$ conversion to TPA) is removed by the evaporative cooling of the solvent (see Chapter3, section 3.1.2). The liquid level is maintained at about $70 \%$ of the reactor volume to provide sufficient vapor disengaging space. The exit gas oxygen concentration is limited to approximately $3 \mathrm{~mol} \%$ to prevent the formation of explosive vapor phase mixtures [20]. Acetic acid combustion rate is estimated to be $5.4 \mathrm{wt} \%$ of the acetic acid fed, resulting in significant solvent loss owing to $\mathrm{CO}_{2}, \mathrm{CO}$ and methyl acetate 
formation (most of the methyl acetate formed from the oxidation reactor is converted to acetic acid via ion exchange catalyst and recovered).

\section{Solid-liquid separation}

The reactor effluent flows through three-stage flash crystallizers (refer to Table $\mathrm{C} 1$ in Appendix $\mathrm{C}$ for operating conditions), each of which has a condenser and a separator. The slurry from the third crystallizer is sent to a centrifuge, where the mother liquor containing the dissolved impurities in acetic acid are separated from crude TPA crystals. Approximately $20 \%$ of the heavies (acetic acid) from the solvent dehydration column (described in a later section) is diverted to the centrifuge to wash the crude TPA crystals. After leaving the centrifuge, the crude TPA is then sent to a dryer to remove the volatile acetic acid, yielding dry crude TPA, which contains about 1,400 ppmw 4-CBA and 600 ppmw $p$-TA. Over the past several decades, efforts have been made to eliminate the drying step prior to purification by hydrogenation (described in a subsequent section) in order to reduce the capital and operating costs $[27,28,132]$. The related alternative technology is to introduce the crude TPA from the centrifuge into a rotary drum filter

or belt filter and pressurize with water to remove the acetic acid (pressure filtration). More particularly, a filter cake is built up through the rotary drum filter or belt filter and the filter cake is covered with a layer of water, and then the water is pressured through the cake to displace the acetic acid.

\section{Catalyst recovery}

Most of the mother liquor from the centrifuge is fed to a reboiled stripper, in which almost all of the acetic acid is vaporized and the heavy ends containing the uncrystallized TPA, intermediates and catalysts are formed in the bottom. The heavies are pumped to the catalyst recovery unit, which is not simulated in this work because this would be common to both of the 
MC process and the spray process. Briefly, the heavies containing the catalysts from the reboiled stripper are sent to an incinerator where the metal acetate catalysts are converted to metal oxide by pyrolysis. Then the metal oxides are converted to metal acetate tetrahydrates by reaction with hydrazine and acetic acid [135].

\section{Off-gas treatment}

In addition to nitrogen, oxygen and combustion gases $\left(\mathrm{CO}_{2}\right.$ and $\left.\mathrm{CO}\right)$, the exhaust gas from the oxidation reactor contains significant amounts of acetic acid and methyl acetate, and minor amounts of unreacted $p \mathrm{X}$ and methyl bromide. A high-pressure scrubber is used to recover acetic acid by countercurrent contact with a water stream derived from the overhead stream of the dehydration column [20]. The scrubber has a bed of cation exchange resin that converts most of the methyl acetate into acetic acid and methanol. In other words, the scrubber is used to recover acetic acid from other VOCs. The acetic acid is then sent to the solvent dehydration column for recycle while the VOCs are let to a fixed bed combustion reactor [136] in which the VOCs are burned to $\mathrm{CO}_{2}$ and water over a supported palladium catalyst. The high temperature treated gas (above $400{ }^{\circ} \mathrm{C}$ ) then passes through an expander where the energy content of the gas is converted to mechanical power that is used to either drive one of the air compressors or for electric power generation. Before being vented to the atmosphere, the effluent gas from the expander is usually fed to a scrubber (not considered in this work) to remove the residual bromine components by countercurrent contact with a caustic solution [136].

\section{Acetic acid dehydration}

The overhead vapor from the reboiled stripper, the liquid and uncondensed streams from crystallizer separators, the bottom stream from the high-pressure scrubber and the vaporized solvents from the dryer are all fed to a distillation column to remove the water generated in the 
oxidation reactor. About $80 \%$ of the dehydrated acetic acid $(\sim 98 \mathrm{wt} \%)$ is then recycled to the oxidation reactor. Azeotropic distillation is generally used for acetic acid/water separation and methyl acetate is used as the entrainer in this case. Water and the entrainer are distilled overhead and separated in a decanter, with only the organic phase being totally refluxed to the column [134].

\section{Hydrogenation unit}

The crude TPA from the oxidation section is mixed with the recycled steam from the solvent evaporator (described in a later section) and the recycled water to form a slurry of about $20 \mathrm{wt} \%$ TPA in water [133]. The slurry is pumped to a dissolver that operates at $267^{\circ} \mathrm{C}$ and 63 bar and is agitated for approximately 15 minutes to dissolve all of the TPA in water. The liquid is then pumped to a hydrogenation reactor in which 4-CBA is converted to $p$-TA by $\mathrm{H}_{2}$. The hydrogenation reactor has two catalyst beds: a top (main) $\mathrm{Pd} / \mathrm{C}$ catalyst bed and a bottom (smaller) $\mathrm{Rh} / \mathrm{C}$ catalyst bed [137]. The TPA/water solution leaving the hydrogenation reactor flows through two-stage crystallizers, a centrifuge and then to a final stage of crystallization followed by a rotary vacuum filter and a PTA (purified TPA) dryer. The dry PTA includes 7 ppmw 4-CBA and 100 ppmw $p$-TA.

A major problem associated with the crystallization recovery process is the potential contamination of the purified TPA crystals with $p$-TA. Shock or sudden cooling is avoided and gradual cooling is achieved in a series of crystallizers to minimize the co-precipitation of the impurities. A subsequent "water reslurry" step is used to remove the impurities (mainly $p$-TA) dissolved in the mother liquor, and thus the $p$-TA content in the purified TPA is lowered to 100 ppmw. It should be noted that the previously described pressure filtration technology can also be applied to effect solid-liquid separation for obtaining purified TPA. This technology can 
eliminate the need for a second stage of reslurrying and filtration of the purified TPA crystals $[27,28]$.

About $51 \%$ of the mother liquor from the centrifuge is recycled to the hydrogenation reactor such that the $p$-TA concentration in the feed is about $3000 \mathrm{ppmw}$ (assumed basis). The rest of the mother liquor is concentrated in the solvent evaporator and the heavy-ends are recycled to the oxidation reactor, in which the $p$-TA is converted back to TPA. Approximately half of the steam from the top of the solvent evaporator is directly mixed with the crude TPA slurry and the rest is used to heat up the reslurry water.

\subsubsection{CEBC spray process}

The spray process layout looks similar to the MC process oxidation section. Instead of a CSTR in the MC process, the oxidation reaction occurs in a spray reactor in which the liquid phase containing dissolved $p \mathrm{X}$ and the catalyst (cobalt acetate, manganese acetate and hydrogen bromide) in acetic acid is dispersed as fine droplets by a nozzle into a continuous vapor phase containing the oxidant $\left(\mathrm{O}_{2}\right)$. Compressed air is used as the oxidant in the simulation of the spray process and the $\mathrm{O}_{2} / p \mathrm{X}$ molar feed ratio is 3.5:1. Note that equimolar $\mathrm{CO}_{2} / \mathrm{O}_{2}$ gas mixture rather than air was used in the laboratory-scale spray reactor. It has been demonstrated that the use of equimolar amounts of $\mathrm{CO}_{2}$ and $\mathrm{O}_{2}$ and excess acetic acid in the vapor phase falls outside the flammability envelope under the experimental conditions (section 3.1.2). It must be noted that using air as the oxidant source in the spray reactor also falls outside the flammability envelope. In the $\mathrm{MC}$ process, typical vapor phase $\mathrm{O}_{2}$ partial pressure is about 0.4 bar [98] or approximately $3 \mathrm{vol} \%$ in the MC oxidation reactor to prevent explosions. In the HYSYS simulation of the spray reactor, $\mathrm{O}_{2}$ concentration in the off-gas is also limited to $3 \mathrm{~mol} \%$. In addition, the acetic acid amount employed in the spray process is more than or equal to that in the $\mathrm{MC}$ process, resulting 
in a higher acetic acid composition in the vapor phase. Given that the acetic acid is above the upper flammability limit in the MC oxidation reactor, it follows that the acetic acid should also be above the flammability limit in the spray reactor.

The reaction temperature and pressure are very close to those in the MC oxidation reactor, i.e., $200{ }^{\circ} \mathrm{C}$ and 15 bar, respectively. As outlined in Table 5.2, four alternative spray processes using varying amounts of acetic acid in the feed are simulated with Case 1 having the highest acid feed rate (10-fold more than that in the MC process) and Case 4 having the least (acetic acid feed rate comparable to that in the $\mathrm{MC}$ process). In all of the four spray cases employed in the simulation, the acetic acid combustion is assumed to be $1.3 \mathrm{wt} \%$ of what is fed. This assumption is based on our preliminary experimental results from the $700 \mathrm{~mL}$ semi-batch spray reactor showing that the $\mathrm{CO}$ formed is approximately one-fourth compared to the MC process (section 3.2.5.4). The reactor product stream flows through three-stage crystallizers, a centrifuge and then a dryer to

yield dry TPA, which is composed of 23 ppmw 4-CBA and 2 ppmw $p$-toluic acid. The purity of the solid TPA meets the polymer-grade TPA requirement and therefore no further purification was deemed necessary. The off-gas treatment part and the distillation part are similar to those employed in the MC process oxidation.

\subsection{Economic Analysis}

\subsubsection{Capital investment}

\subsubsection{Purchased equipment costs}

The estimates of purchased equipment costs are determined by equipment parameters such as equipment size, weight, surface area, and material of construction $[129,130]$ that are in turn based on the material and energy balances obtained from HYSYS simulation. Cost estimation 
details including equipment design parameters and correlating equations for both processes are provided in Tables D1 \& D2 of Appendix D. All the costs are adjusted to June 2012 figures using Chemical Engineering Plant Cost Index (CEPCI) [138]. Figure 5.3 shows the purchased costs (f.o.b., free on board, meaning that the purchaser pays the freight) of major equipment in both processes. Special equipment costs such as crystallizers, solid-liquid separation units, dryers and storage silos account for about $40 \%$ of the costs in both processes. Miscellaneous equipment costs mainly consist of the $\mathrm{Pd}$ and $\mathrm{Rh}$ catalysts for the hydrogenation reaction in the $\mathrm{MC}$ process.

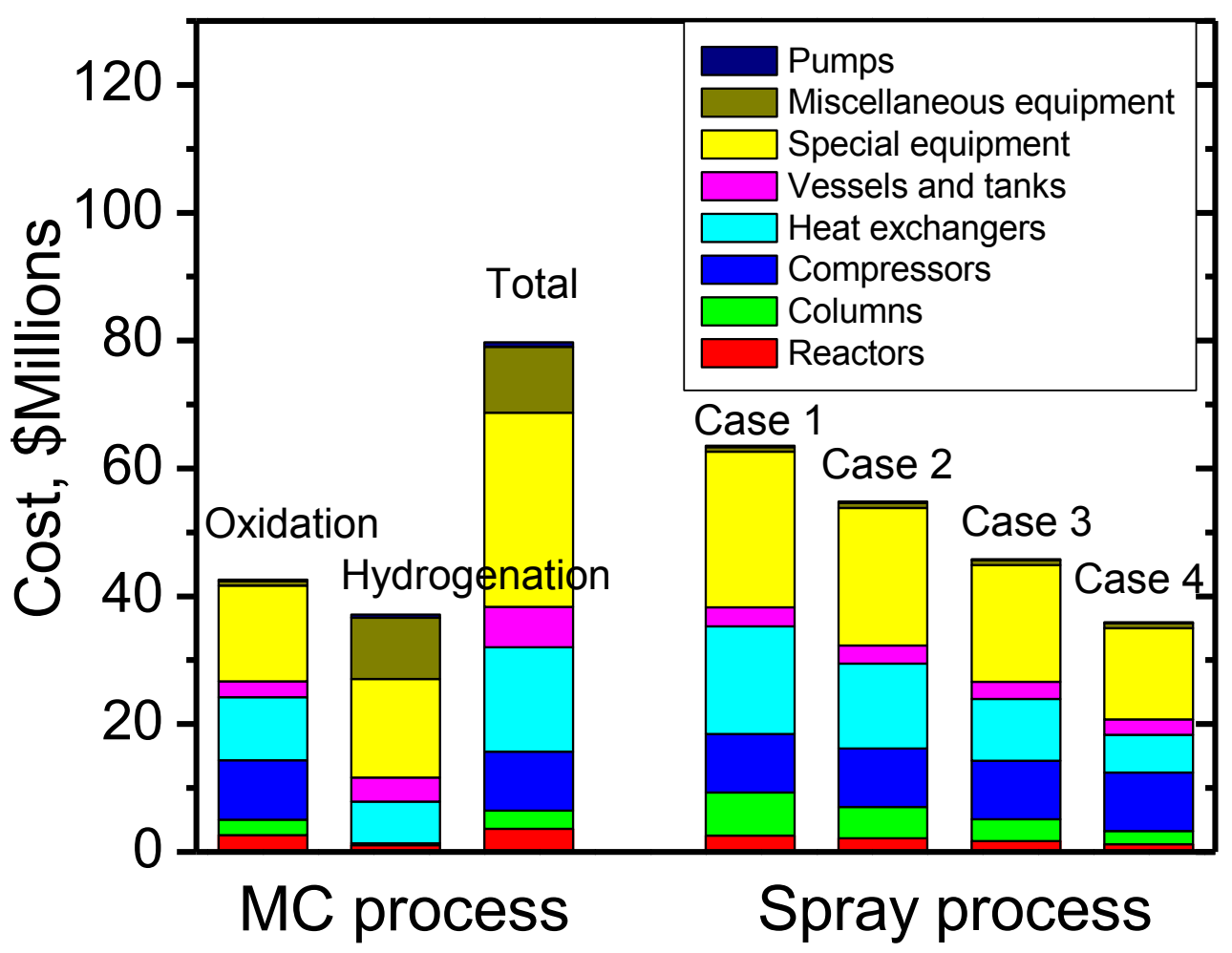

Figure 5.3: Comparison of purchased equipment costs for $\mathrm{MC}$ and spray processes

\subsubsection{Estimate of capital investment}

The estimate of capital investment is based on the "purchased equipment cost" method, in which the purchased equipment cost is treated as the basis, and other cost (direct, indirect and 
working capital) are estimated by multiplying the purchased equipment cost with a corresponding factor [129]. Table 5.3 compares the capital investments for both MC and spray processes. The estimated total capital investments of the spray process case 1 ( $\$ 241$ million when nearly 10 times more acetic acid is used in the feed compared to the MC process) and case 4 (\$136 million when similar amounts of acetic acid feed is used as in the MC process) are approximately $80 \%$ and $45 \%$, respectively, of those estimated for the MC process ( $\$ 302$ million). Thus, the capital cost benefit margin in the CEBC spray process (due to non-requirement of hydrogenation section) is progressively diminished from $55 \%$ at case 4 to $20 \%$ at the highest acetic acid throughputs of case 1 .

Table 5.3: Comparison of capital costs for both MC and spray processes

\begin{tabular}{lccccc}
\hline & $\begin{array}{c}\text { MC Process } \\
\text { (\$Million) }\end{array}$ & Case 1 & Case 2 & Case 3 & Case 4 \\
\cline { 2 - 6 } Purchased equipment costs (f.o.b.) & 79.7 & 63.6 & 54.8 & 45.8 & 35.9 \\
Direct installation costs & 98.1 & 78.2 & 67.4 & 56.4 & 44.2 \\
Direct costs (others) & 35.9 & 28.6 & 24.7 & 20.6 & 16.2 \\
$\quad$ Total direct costs & 213.8 & 170.4 & 146.9 & 122.9 & 96.3 \\
Indirect costs & 58.2 & 46.4 & 40.0 & 33.5 & 26.2 \\
$\quad$ Fixed capital investment (FCI) & 272.0 & 216.8 & 186.9 & 156.3 & 122.6 \\
Working capital & 30.2 & 24.1 & 20.8 & 17.4 & 13.6 \\
\hline \multicolumn{1}{c}{ Total capital investment (TCI) } & 302.2 & 240.9 & 207.7 & 173.7 & 136.2 \\
\hline
\end{tabular}

\subsubsection{Total production cost}

\subsubsection{Utilities and raw material costs}

The utilities and raw material costs (variable costs) for both MC process and spray process are summarized in Table 5.4. The $p$-xylene makes up the bulk of the raw material costs for both processes. Steam and electricity, used mainly in the heat exchangers and special equipment, 
compressors and pumps, account for the large part of the utilities. Note that the estimated utility costs for the MC process ( $\$ 7.37 / \mathrm{lb}$ of TPA) are more compared to even case 1 of the CEBC process ( $₫ 6.46 / 1 \mathrm{~b}$ of TPA), wherein maximum energy input is needed to handle the higher acetic acid throughputs (cases 1-3). 


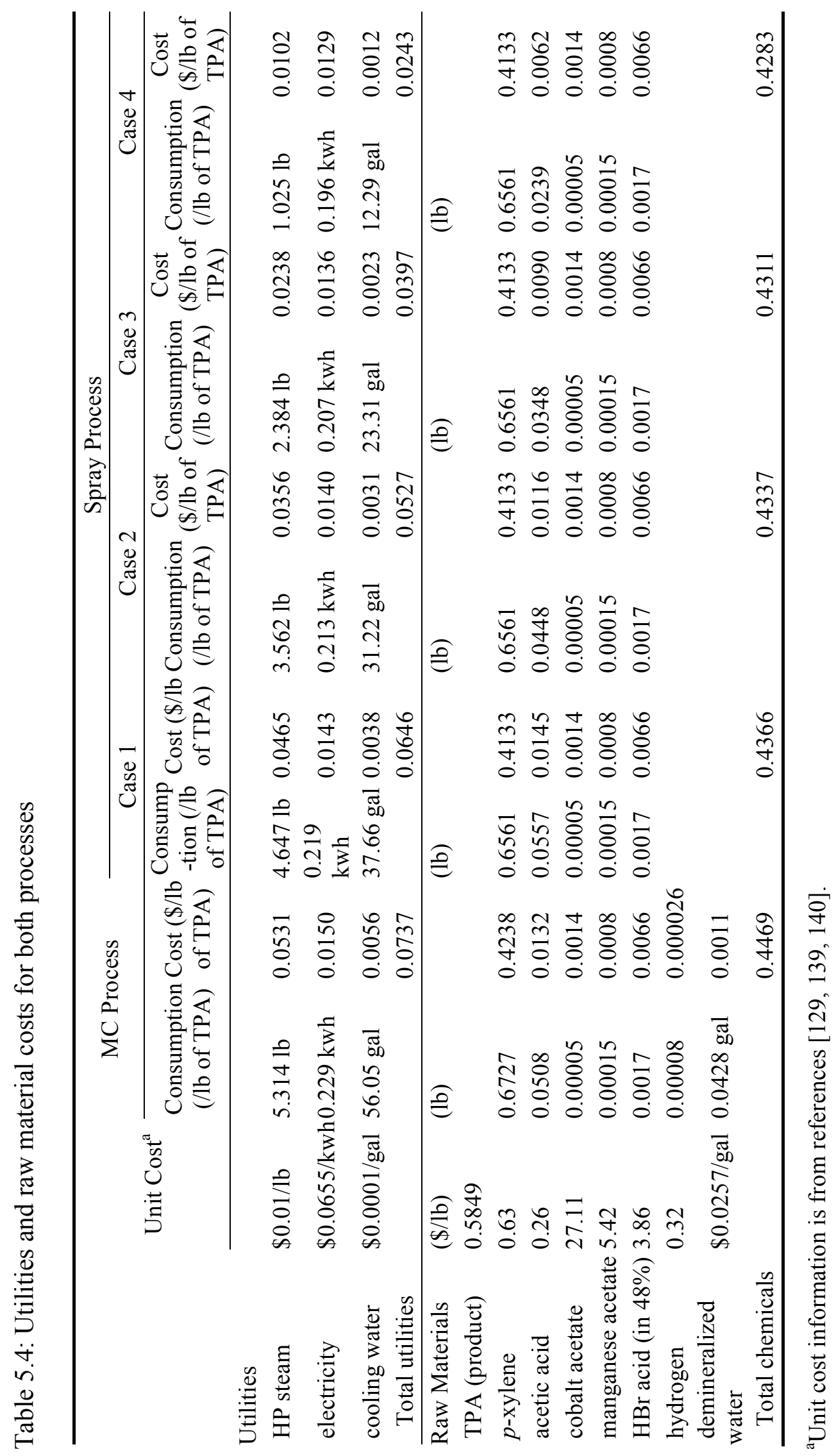




\subsubsection{Estimate of total production cost}

Figure 5.4 compares the total product cost for both processes (cost details of each item are provided in Table D3 of Appendix D). The total cost of the polymer-grade TPA product from the MC process is estimated to be $\$ 0.67 / \mathrm{lb}$. The total TPA cost from CEBC process case 1 and case 4 is estimated to be $\$ 0.635 / \mathrm{lb}$ and $\$ 0.56 / \mathrm{lb}$, respectively.

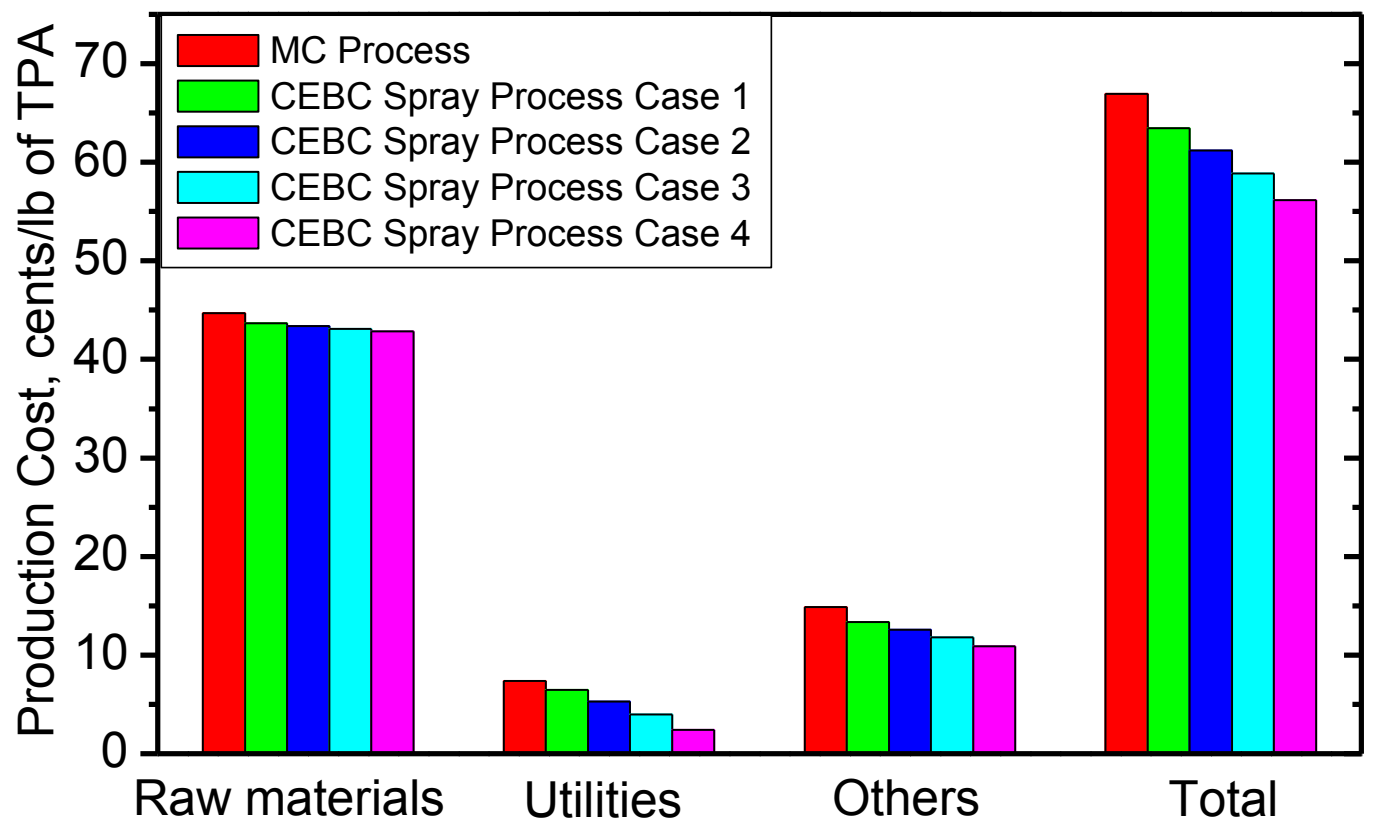

Figure 5.4: Comparison of total production costs for both processes

(Others include other direct, indirect costs, depreciation and general expenses such as labor, research, plant overhead, operating supplies and maintenance, details are provided in Table D3 of Appendix D)

\subsubsection{Sensitivity analysis}

Using lower $p \mathrm{X}$ concentration (higher acetic acid throughput) partly offsets the economic benefits accrued by the elimination of the hydrogenation step in the CEBC spray process. Figure 5.5 plots the capital and operating costs of the CEBC process as a function of acetic acid $/ p \mathrm{X}$ 
weight ratio. The capital investment and production cost of the spray process can increase by $120 \%$ and $13 \%$ respectively when acetic acid amounts increase nearly 10 -fold, and this change is linear. Clearly, as high a $p \mathrm{X}$ concentration as practically feasible should be used to maximize the savings in the CEBC spray process.

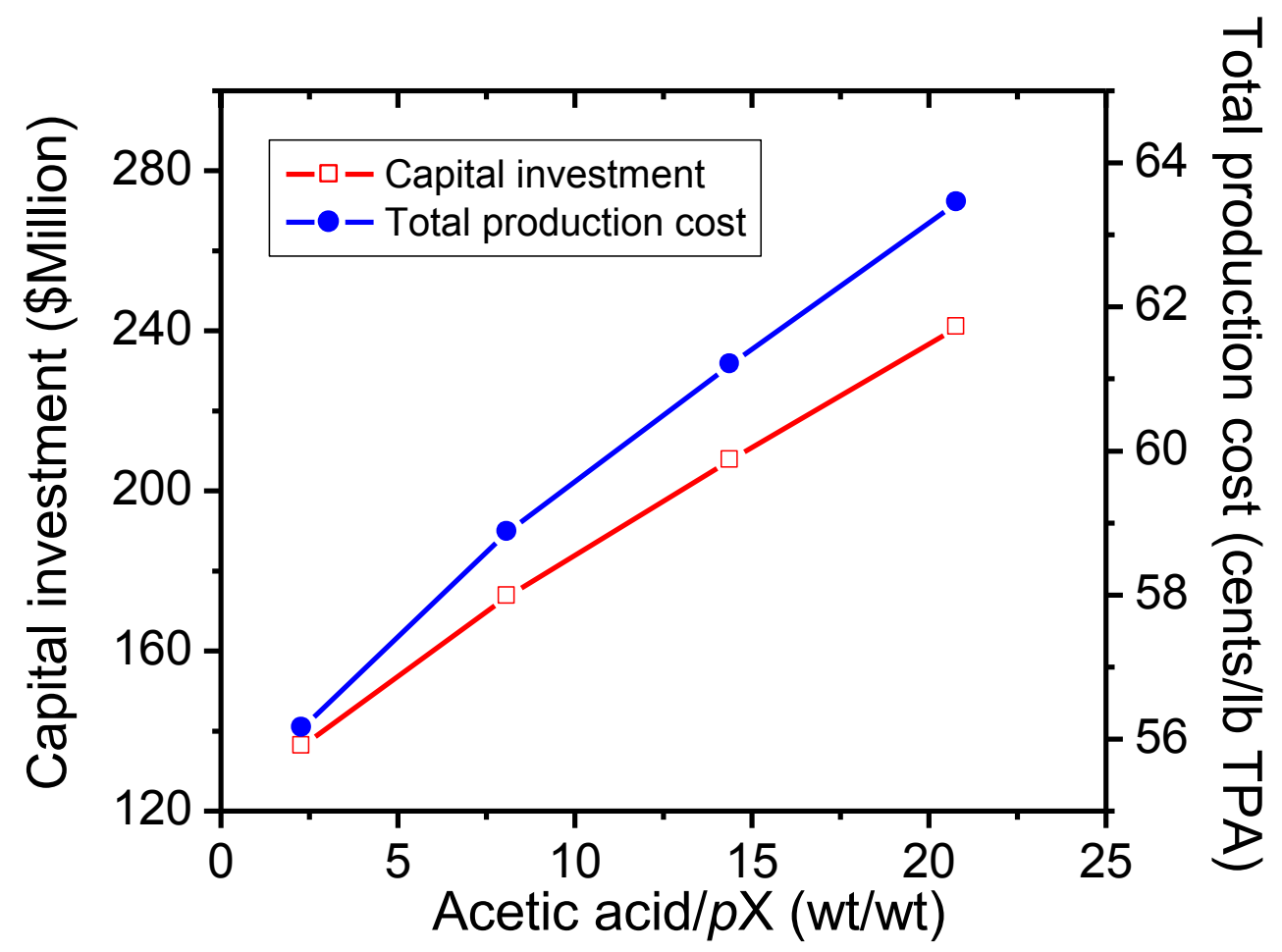

Figure 5.5: Capital and operating cost rise with acetic acid throughput in CEBC spray process

\subsection{Environmental Impact Analysis}

A sustainable process should demonstrate not only clear economic advantages, but also superiority in terms of of environmental stewardship and social progress [141, 142]. To this end, comparative environmental impacts of the $\mathrm{MC}$ and spray processes are evaluated in this section. There are several techniques for environmental assessment such as input-output analysis, environmental impact assessment (EIA), environmental impact indices, environmental risk assessment and life cycle assessment (LCA) [143]. Among the various environmental assessment 
approaches, LCA has become increasingly attractive and widely adopted in environmental impact analysis for making environmental decisions.

\subsubsection{Introduction to LCA}

LCA is a developing scientific methodology for identifying, quantifying and assessing environmental impacts throughout the life cycle of a product, process, or activity. The full LCA considers the material and energy uses and releases to the environment of the entire life cycle from cradle-to-grave (from raw material extraction through manufacturing, use and disposal phase) [144]. In fact, the user is free to select any of the following type of LCA: cradle-to-gate (from raw material extraction to factory gate, i.e., before transported to the consumer), gate-togate (an assessment when the product is at the manufacturing facility), gate-to-grave (evaluation of the environmental impact when the product leaves manufacturing facility to final product disposal).

Efforts have been made to develop and standardize the LCA methodology for decades, with inputs from important active organizations including the Society for Environmental Toxicology and Chemistry (SETAC), the United Nations Environmental Program (UNEP) and the International Organization for Standardization (ISO) [145-147]. An international standard for LCA developed by ISO suggests the four main phases in LCA in ISO 14040 series: a) goal and scope definition (ISO 14040); b) life cycle inventory (ISO 14041); c) life cycle impact assessment (ISO 14042) and d) life cycle assessments and interpretation (ISO 14043). [148, 149] Since the standard must be applicable to many industrial and consumer sectors, it is rather general. Nonetheless, it includes a comprehensive set of terms and definitions, a methodological framework for each of the four components, reporting considerations, approaches for critical review, and an appendix describing the application of LCA [150]. 
One key question during the practical application of LCA to processes is how to translate the inventory results of material, energy, waste and toxic emissions into category indicators for directly assessing the environmental burdens. There are several category-specific characterization models such as TRACI (the Tool for the Reduction and Assessment of Chemical and other environmental Impacts), CML 2001 [CML stands for Centrum voor Milieuwetenschappen (Dutch: Leiden University Institute of Environmental Sciences)] and Ecoindicator 99 to accomplish this translation efficiently. Generally, these LCA models can be grouped into two categories: mid-point approaches (problem-oriented approach or classical impact assessment method) which translates the category impact into real phenomenon such as acidification and ozone depletion and end-point approaches (damage-oriented approach) which translates the category impact based on the area of protection such as human health, natural resources and natural and human made environment in a cause-effect chain or network [151]. TRACI, CML 2001, EDIP 97 are typical of mid-point models while Eco-indicator 95 and 99, EPS 92, 96 and 2000 and LIME 2003 are examples of end-point approaches [151].

LCA is a powerful tool used for collecting and analyzing inventory data, weighing and ranking the environmental burdens of products, processes, or activities in a transparent and scientific way. It can help identify the hot spots that cause the greatest environmental impacts of the life cycle and thus provide decision makers with the resulting information to make improvements. It can also offer a link between the environmental impacts and economics of processes to help select an optimized application. CEBC researchers have employed LCA methodology for performing comparative environmental impacts of process concepts (ethylene and propylene epoxidations) developed at CEBC with conventional processes [127, 128].

\subsubsection{Methodology}


Comparative gate-to-gate LCA and cradle-to-gate LCA of the conventional MC process and the CEBC spray process (4 cases) were performed. The approach employed in this analysis follows the four phases (goal and scope definition, life cycle inventory, impact assessment and interpretation) suggested by ISO. Figure 5.6 depicts the methodological framework for LCA of the TPA processes. Life cycle inventory database of the material and energy flows associated with the conventional and CEBC processes was provided by the Aspen HYSYS simulations which were also used for performing the comparative economic analysis. The life cycle impact assessment was accomplished using GaBi 6 software [152] developed by PE International jointly with University of Stuttgart LBP (former IKP). TRACI, incorporated in $\mathrm{GaBi}^{\circledR}$ with U.S. professional and extension databases, was used to translate the inventory results into category indicators for environmental impact assessment.

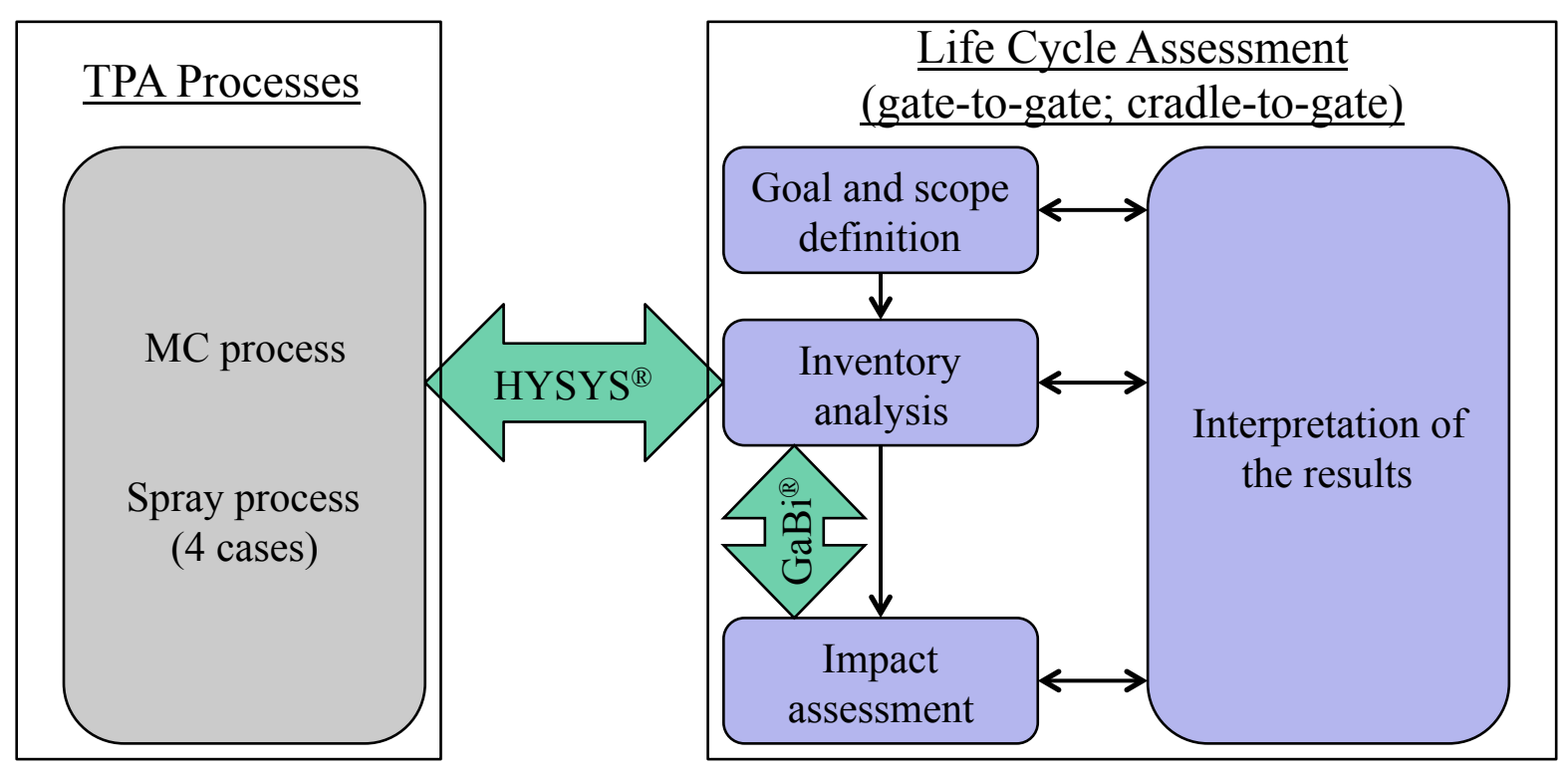

Figure 5.6: Methodological framework for LCA of TPA processes

$\mathrm{GaBi}^{\circledR}$ is a commercial LCA software tool for product sustainability solution by creating life cycle balances. This software is accompanied by an inventory database in compliance with the ISO 14044, ISO 14064 and ISO 14025 standards. For example, the datasets cover thousands of 
production processes such as chemicals, power generation and transport over the supply chain of the represented cradle-to-gate inventory according to individual country/region specific situation. $\mathrm{GaBi}^{\circledR}$ incorporates various LCA models such as TRACI, CML 2001 and Eco-indicator 99. While most of these models have been developed for use in specific country/region particularly in Europe or Japan, TRACI was developed by United States Environmental Production Agency (US EPA) in 2002. The underlying methodologies in TRACI reflect state-of-the-art developments for LCA in the US, and are using input parameters that are specific to US locations [153-156]. TRACI facilitates the characterization of the following environmental categories: ozone depletion, global warming, acidification, eutrophication, tropospheric ozone (smog) formation, ecotoxicity, human particulate effects, human carcinogenic effects, human non-carcinogenic effects, fossil fuel depletion, and land use effects. Consistent with EPA'S decision not to aggregate between environmental impact categories, many of the impact assessment methodologies within TRACI are based on mid-point characterization approaches [154].

In order to validate the methodologies employed in the Aspen HYSYS simulations as well as in the $\mathrm{GaBi}^{\circledR}$ LCA software, a gate-to-gate environmental impact assessment of the simulated conventional MC process was first performed and the predictions were compared with the total and released chemical wastes and green house gas emissions from a commercial plant (BP Amoco Chemical Company Cooper River Plant). The actual commercial plant emission data are available from public databases such as the annual toxic release inventory data reported to US EPA [157] and EPA's facility level information on Green House Gases Tool [158].

Fugitive emissions (unintentional releases of process fluid from equipment and evaporation of volatile liquids from open areas) are an important environmental intervention [143]. It was 
reported that $70-90 \%$ of air emissions for some plants in the United States resulted from the fugitive emissions [159]. Siegell [160] also claimed that in almost all cases, fugitive emissions from equipment leaks were the largest source of volatile organic compounds (VOCs) emissions in refineries, typically accounting for $40-60 \%$ of total VOC emissions. However, $\mathrm{GaBi}^{\circledR}$ fails to reveal these emissions from mass and energy balances based on process flowsheets for the LCA study. To the best of our knowledge, most LCA software tools don't include estimates for fugitive emissions. To execute a thorough gate-to-gate environmental impact analysis, the method used for gate-to-gate emission estimation was based on the Environmental Fate and Risk Assessment Tool (EFRAT) developed by Shonnard and Hiew [161]. Specifically, the emission rates from process unit operations were calculated by the throughput of VOCs in each piece of equipment multiplied by the average emission factors for that particular type of chemical process unit. Emission sources considered in the gate-to-gate environmental impact analysis include the unit operations such as reactors, distillation columns and strippers, the fugitive emissions from valves, flanges and pump seals, as well as the fuel combustion emissions for utility production.

The estimate of utility emissions was based on the $\mathrm{GaBi}^{\circledR}$ database. In the cradle-to-gate environmental impact analysis, the fugitive emissions would also be considered.

\subsubsection{Environmental impact analysis of TPA processes}

\subsubsection{Goal and scope definition}

\section{Goal}

The major project goal is to quantitatively benchmark the environmental impacts of TPA production by the CEBC spray process against the conventional MC process. Such a comparison 
should provide a clear picture of potential environmental advantages of the spray process and also identify the hot spots and opportunities for improvements.

Scope definition (LCA assumptions and system boundaries)

\section{Functional unit}

Functional unit for this analysis is the production of $63450 \mathrm{~kg} / \mathrm{h}(500,000$ metric tons/year) of purified TPA (i.e., polymer-grade TPA quality specifications) by both the conventional MC process and the CEBC spray process.

\section{System boundaries}

The scope of the evaluation is limited to gate-to-gate and cradle-to-gate analyses. The use and disposal phases are not considered. For gate-to-gate analysis, the system boundaries of both processes are limited to factory entry gate to exit gate (Figure 5.7). In other words, only the environmental impacts during TPA manufacture at the production facility stemming from emissions from unit operations, fugitive emissions, and utility emissions are considered. Electricity is assumed to be obtained from U.S. power grid from a portfolio of energy sources. Steam is assumed to be produced from natural gas. In contrast, the cradle-to-gate analysis assessment includes gate-to-gate scope and also the environmental impact from the extraction, manufacture and transportation of the raw materials. 


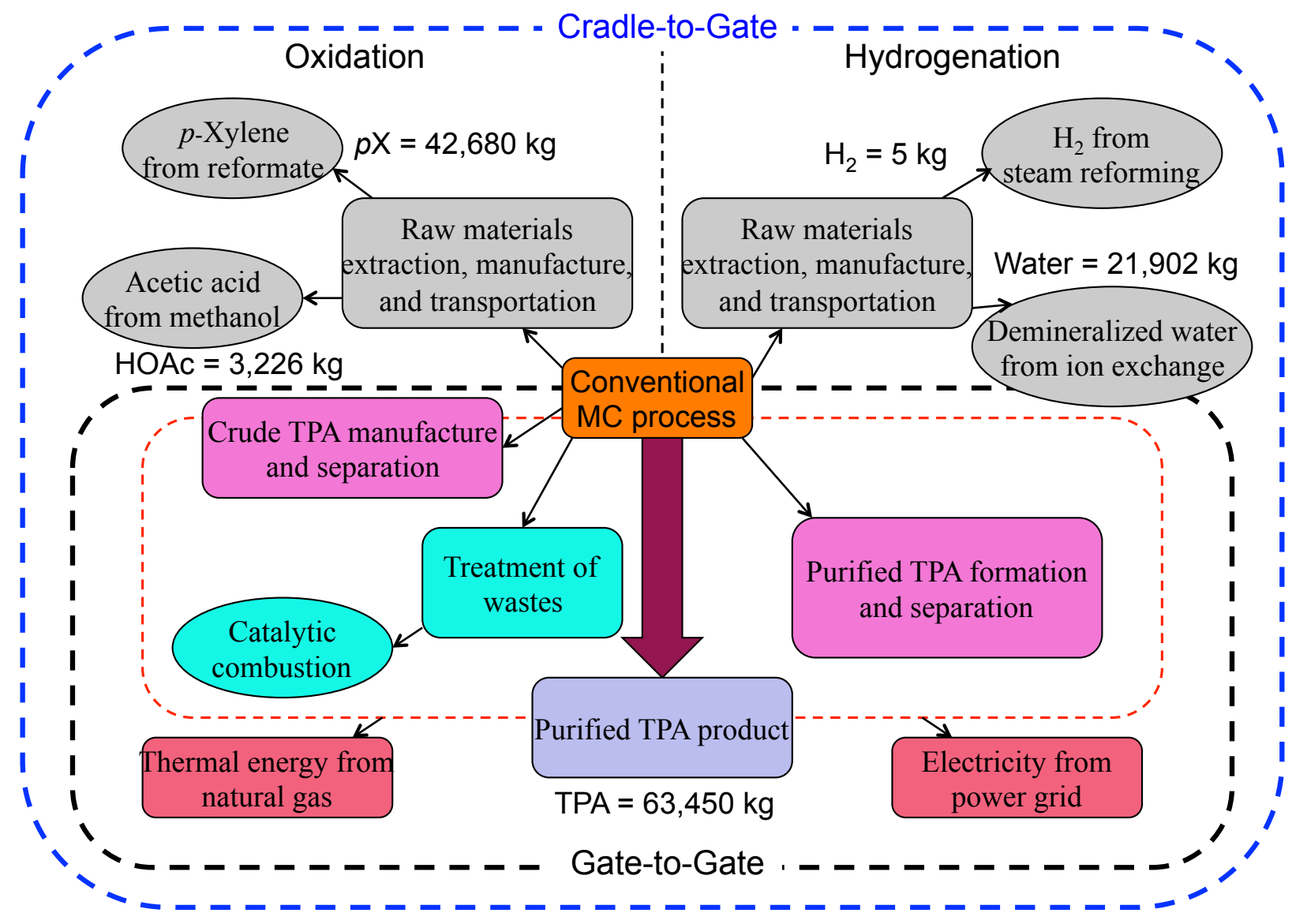

Figure 5.7a: System boundaries of conventional MC process

for gate-to-gate and cradle-to-gate LCA 


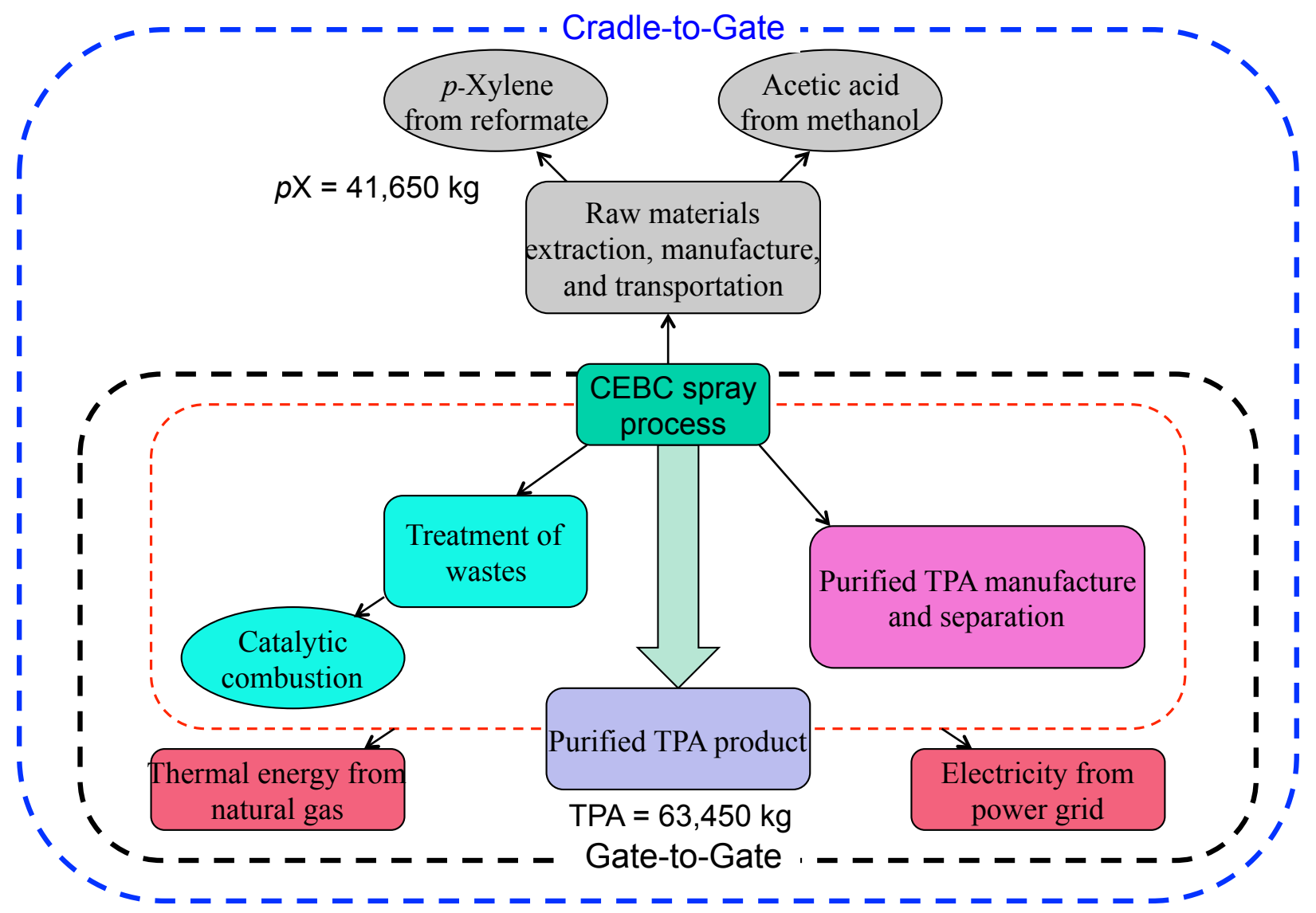

Figure 5.7b: System boundaries of CEBC spray process

for gate-to-gate and cradle-to-gate LCA

\section{Raw material sources}

The main raw materials used in the $\mathrm{CEBC}$ spray process and $\mathrm{MC}$ process oxidation section are $p$-xylene and acetic acid. The production of cobalt acetate, manganese acetate and hydrobromic acid is not considered since the usage amounts of these catalysts are small compared to the other raw materials. Hydrogen and demineralized water are also important raw materials for the $\mathrm{MC}$ process hydrogenation section. Besides, the environmental impacts due to energy production such as electricity and steam from fossil fuel sources are also considered.

Petroleum is practically the only natural source of xylenes. $p$-Xylene is primarily separated from reformates, in which naphtha fractions (from naphthenic crude oils or produced by oil 
hydrocracking) are used as the raw materials for the catalytic reforming process [162]. The $\mathrm{C}_{8}$ and heavier aromatics $\left(\mathrm{C}_{8+}\right.$ fraction) produced from the reformates pass through a distillation column where $p$-xylene is first separated (from $o$-xylene and $\mathrm{C}_{9+}$ aromatics. $m$-Xylene, which has a nearly identical boiling point to $p$-xylene, is mostly isomerized to $o$ - and $p$-xylenes before fed to the distillation column). The $p$-xylene is further purified from the isomer mixture by adsorption on a molecular sieve [163].

The major synthetic routes to acetic acid are methanol carbonylation and liquid-phase oxidation of butane, naphtha, or acetaldehyde, with methanol carbonylation being the preferred route for large-scale production [164]. A rhodium catalyst is usually used for the one-step methanol carbonylation process, in which methanol and carbon monoxide are fed into a stirred reactor and the reaction is conducted at $200{ }^{\circ} \mathrm{C}$ and 35 bar [165]. Methanol is generally produced from synthesis gas, which in turn is produced from natural gas by either steam reforming or partial combustion.

Steam reforming of natural gas or heavy fuel oil is the main technology used for the industrial scale production of hydrogen [166]. Electrolysis of water and hydrocracking of hydrocarbons are relatively minor sources of industrial hydrogen. In steam reforming, the natural gas or heavy fuel oil and steam are passed over a nickel-based catalyst to form hydrogen and carbon monoxide.

There are usually three methods to obtain demineralized water: distillation, ion exchange and reverse osmosis [167]. Ion exchange, in which cations and anions are exchanged with protons and hydroxide ions, is modeled in the GaBi dataset.

In the GaBi dataset, the electricity used is modeled according to the individual countryspecific situation. Specifically in our case, the power plants in service are based on the US power grid from a portfolio of energy sources. In this model, the net losses and imported electricity as 
well as the import, transport, mining and exploration processes for the energy carrier supply chain are all considered. The steam used for heat exchangers is assumed to be produced from natural gas.

\section{Allocation}

Since no valuable byproducts are obtained from either the MC process or the spray process, the allocation rule need not be considered in this evaluation.

\subsubsection{Life cycle inventory}

For both the MC process and the CEBC process, the inventory of the LCA system includes the inputs and outputs for the processes within the system boundaries (Figure 5.5), including the raw material and energy inputs, products and co-products outputs, and generated wastes released to air, water and soil. In this particular analysis, the foreground inventory data associated with the material and energy balances during TPA production at the facility are identical to those used for the comparative economic analysis (obtained from Aspen HYSYS simulations, refer to Appendix C). The background inventory data associated with raw material extraction, utility production and transportation are obtained from GaBi U.S. professional and extension databases. Flow models for cradle-to-gate LCA consistent with the system boundaries defined in Figure 5.7 are shown in Figure 5.8a (MC process) and Figure 5.8b (CEBC spray process). Flow models for gate-to-gate LCA (not shown here) look similar to those for cradle-to-gate LCA except that the section related to raw material extraction, manufacture and transportation is omitted. 
MC Process

GaBi process plan: Mass [kg]

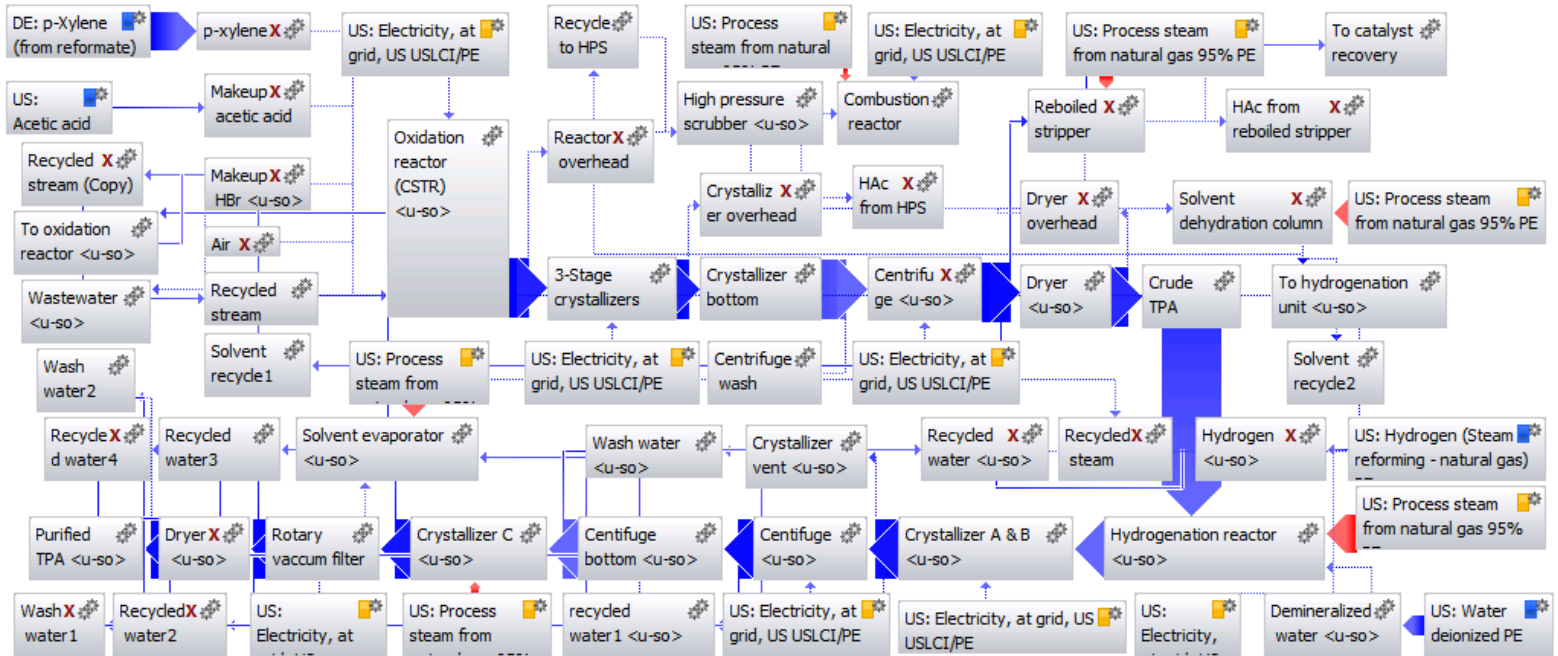

Figure 5.8a: Flow model of conventional MC process for cradle-to-gate LCA simulated in $\mathrm{GaBi}^{\circledR}$

\section{Spray Process Case1}

Gabi process plan: Mass [kg]

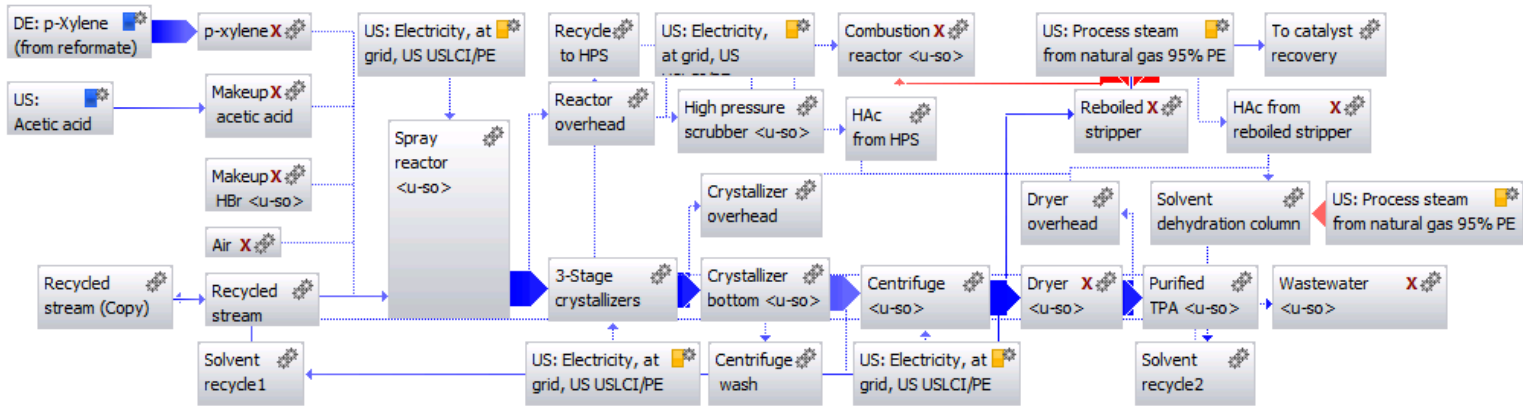

Figure 5.8b: Flow model of CEBC spray process for cradle-to-gate LCA simulated in $\mathrm{GaBi}^{\circledR}$ 


\subsubsection{Life cycle impact assessment}

\section{Methodology validation by gate-to-gate LCA of MC process}

\section{Comparison of VOC emissions}

As described in section 5.5.2, the gate-to-gate VOC emission rate from a unit operation is estimated by the VOC mass flow rate passing through the equipment multiplied by an average emission factor. The industry emission factors for various unit operations are summarized in the textbook by Allen and Shonnard [168] which also provides average emission factors for estimating fugitive emissions from valves, pump seals, flanges and other connections. The estimates of air emissions of two VOCs, $p$-xylene and methanol, are given in Table 5.5. These estimated emissions are compared with the waste quantity report of a commercial TPA plant, namely, BP Amoco Chemical Company Cooper River Plant in South Carolina with an annual production capacity of 1.345 million metric tons of purified TPA [11] The report was obtained from EPA public database [157], and the data therein are also included in Table 5.5.

Table 5.5: Comparison of conventional MC process VOC emissions obtained from U.S. EPA Toxic Release Inventory (TRI) data for commercial facility with $\mathrm{GaBi}^{\circledR}$-estimated emissions

\begin{tabular}{|c|c|c|c|c|c|}
\hline \multirow[b]{2}{*}{ Chemical } & \multicolumn{4}{|c|}{$\begin{array}{l}\text { Reported total and released chemical waste } \\
\text { by BP Amoco Chemical Co., } 2011\end{array}$} & \multirow{2}{*}{$\begin{array}{c}\text { Gate-to-gate } \\
\text { emissions (lb), } \\
\text { this work }\end{array}$} \\
\hline & Total waste & $\begin{array}{l}\text { Adjusted total } \\
\text { waste }^{\mathrm{a}}\end{array}$ & Released waste & $\begin{array}{c}\text { Adjusted } \\
\text { released waste }\end{array}$ & \\
\hline & 1258 & 418,513 & 25,79 & 9,589 & \\
\hline methanol & $1,703,100$ & 633,123 & 103,100 & 38,327 & 298,332 \\
\hline
\end{tabular}

${ }^{a}$ Adjusted wastes corresponded to the simulated annual capacity (500,000 metric tons PTA);

${ }^{\mathrm{b}}$ The TRI reported waste is the chemical waste emissions ( $p \mathrm{X}$ and methanol VOC emissions) to air.

As shown in Table 5.5, the estimated VOC emissions are approximately half of those reported by BP (total wastes). The TRI data are generally higher probably because the BP facility has two 
other chemical production sites in addition to the purified TPA site [11]. Nevertheless, the predicted and reported waste quantities are of the same order of magnitude, implying the method used for the process simulation and gate-to-gate VOC emission estimation is generally satisfactory on this level of analysis. One can also observe from Table 5.5 that more than $90 \%$ of the generated wastes are treated at the facility and the rest of them are released to the atmosphere.

\section{Comparison of $\mathrm{CO}_{2}$ emissions}

Major sources for direct gate-to-gate $\mathrm{CO}_{2}$ emissions to air are $\mathrm{CO}_{2}$ generated from solvent burning in the oxidation reactor, the combustion reactor for VOCs treatment as well as the onsite boilers for steam production by fuel combustion. Table 5.6 compares the estimated $\mathrm{CO}_{2}$ equiv. for the conventional $\mathrm{MC}$ process obtained from $\mathrm{GaBi}^{\circledR}$ with the reported facility $\mathrm{CO}_{2}$ emissions by BP Amoco Chemical Company Cooper River Plant (SC) for year 2011 [158]. Clearly, less than $10 \%$ of the total $\mathrm{CO}_{2}$ emission equivalent is emitted as direct $\mathrm{CO}_{2}$ emissions to air at the facility. The predicted and reported $\mathrm{CO}_{2}$-equiv. quantities are of the same order of magnitude, which provides a measure of the predictive ability of the TRACI tool incorporated in $\mathrm{GaBi}^{\circledR}$.

Table 5.6: Comparison of conventional $\mathrm{MC}$ process $\mathrm{CO}_{2}$ emissions obtained from U.S. EPA $\mathrm{GHG}$ data for commercial facility with $\mathrm{GaBi}^{\circledR}$-estimated emissions

\begin{tabular}{|c|c|c|c|c|}
\hline \multicolumn{2}{|c|}{$\begin{array}{l}\text { Reported total facility } \mathrm{CO}_{2} \text { emissions (in metric } \\
\text { tons } \mathrm{CO}_{2} \text {-equiv.), by BP Amoco Chemical Co., } \\
\qquad 2011\end{array}$} & \multicolumn{3}{|c|}{$\begin{array}{c}\text { Gate-to-gate } \mathrm{CO}_{2} \text { emissions (in metric } \\
\text { tons } \mathrm{CO}_{2} \text {-equiv.), this work }\end{array}$} \\
\hline Reported & Adjusted $^{\mathrm{a}}$ & Off-gas & $\begin{array}{c}\text { Fuel } \\
\text { combustion }\end{array}$ & Total \\
\hline 101,458 & 37,717 & 60,625 & 327,833 & 388,458 \\
\hline
\end{tabular}

${ }^{a}$ Adjusted emissions corresponded to the simulated annual capacity (500,000 metric tons purified TPA). 


\section{Comparative gate-to-gate life cycle impact assessment}

Figure 5.9 compares the gate-to-gate environmental impact potentials of TPA production by the conventional $\mathrm{MC}$ and $\mathrm{CEBC}$ spray processes (only the major impact categories are shown in Figure 5.9). The numerical data and a complete list of all impact potentials are provided in Table E1 of Appendix E. The estimated environmental impact potential of direct process emissions such as off-gas, wastewater, VOCs from unit operations and fugitive emissions (fuel combustion effect for steam production excluded) are also provided in Figure 5.9 (shaded regions). The interpretation of the results will be discussed in detail in next section. As expected, the adverse environmental impact potentials in the CEBC spray process decreases with a lower substrate $(p \mathrm{X})$ concentration, i.e., smaller amounts of acetic acid employed. Table 5.2 copied the HYSYS simulation parameters associated with acetic acid for the $\mathrm{MC}$ process and $\mathrm{CEBC}$ spray process (case 1 to case 4 ).

Table 5.2: Simulation parameters associated with acetic acid for both processes

\begin{tabular}{cccccc}
\hline & MC process & \multicolumn{4}{c}{ Spray process } \\
\cline { 3 - 6 } & Case 1 & Case 2 & Case 3 & Case 4 \\
\hline HAc/pX feed ratio $(\mathrm{wt} / \mathrm{wt})$ & 2.3 & 20.8 & 14.4 & 8.1 & 2.3 \\
$\begin{array}{c}\text { HAc loss through } \\
\text { oxidation reactor }(\%)\end{array}$ & 5.3 & 1.3 & 1.3 & 1.3 & 1.3 \\
\hline
\end{tabular}




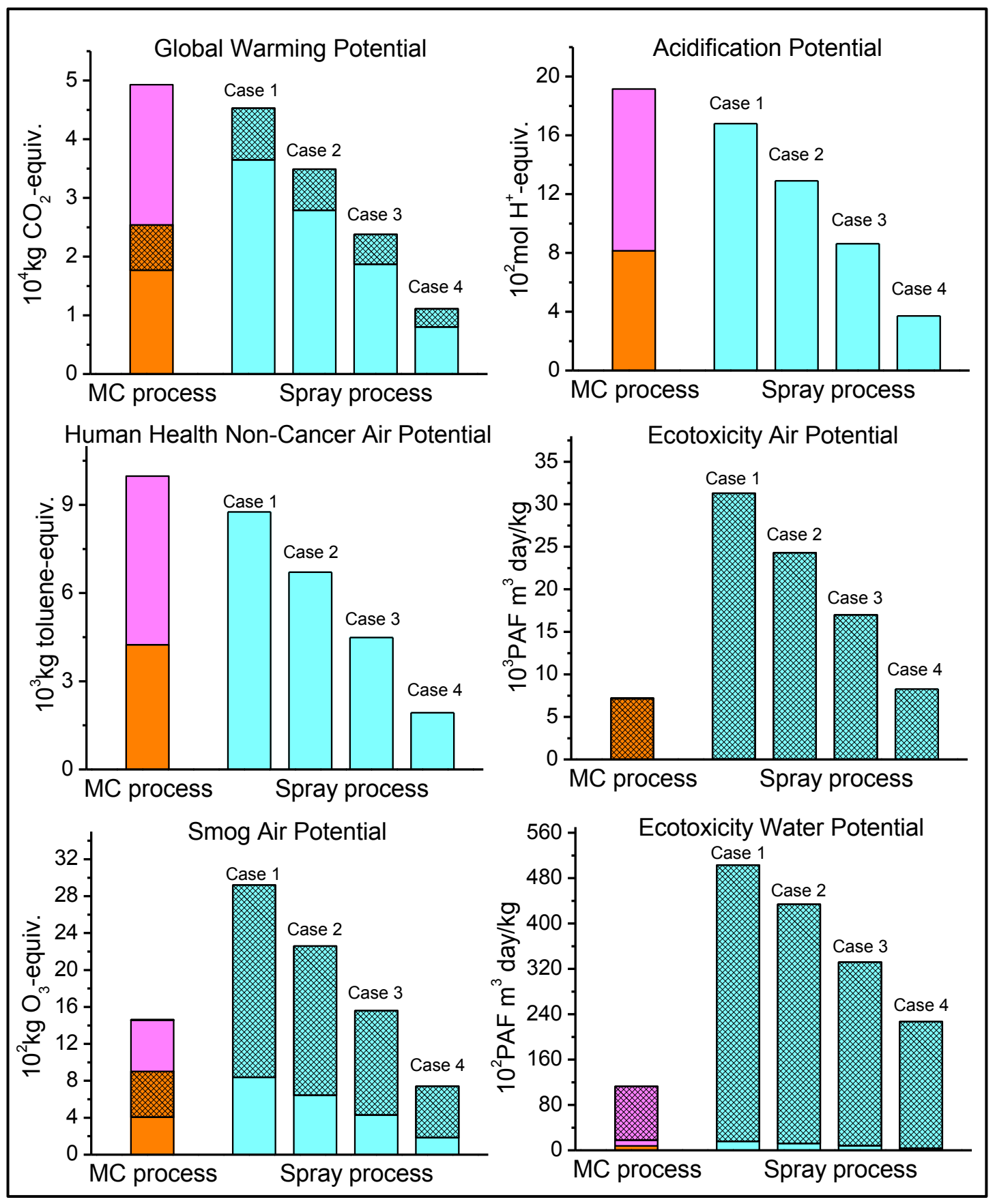

Figure 5.9: Comparison of predicted gate-to-gate environmental impact potential for both processes

(Orange: MC process oxidation section; Magenta: MC process hydrogenation section; Cyan: spray process; Shaded: environmental impact potential of direct process emissions such as offgas, wastewater, VOCs from unit operations and fugitive emissions;

Unshaded: fuel combustion for process steam production) 


\section{Comparative cradle-to-gate life cycle impact assessment}

The predicted cradle-to-gate environmental impact potential for both processes are shown in Figure 5.10. The numerical data and a complete list of all impact potentials are provided in Table E2 of Appendix E. The predicted cradle-to-gate environmental impact potential for most of the impact categories are generally an order of magnitude greater than the gate-to-gate impact potentials, while they are of the same order of magnitude with respect to ecotoxicity air and ecotoxicity water potential, the major contributors to which are VOC emissions to air and the organic solvents contained in wastewater. The contributors to the various environmental impact categories are described in the following sections. 


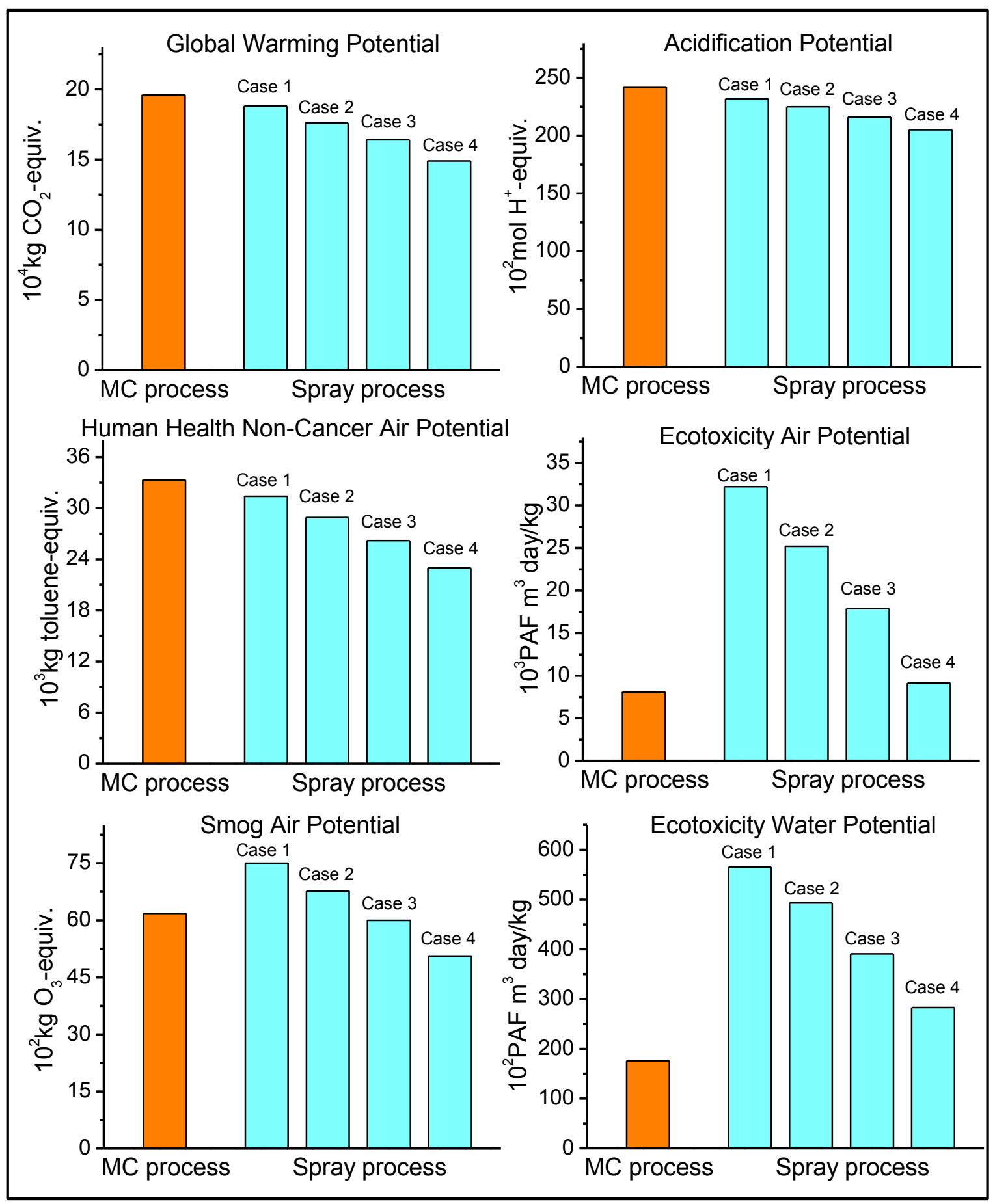

Figure 5.10: Predicted cradle-to-gate environmental impact potential for both processes (Orange: MC process; Cyan: Spray process) 


\subsubsection{Data interpretation}

\section{Environmental impact potential contributors in the TPA processes}

\section{Global warming potential}

Global warming potential arises from $\mathrm{CO}_{2}$ emissions and other greenhouse gases that trap the sun's heat. As shown in Figure 5.9, the $\mathrm{CO}_{2}$ emissions for a TPA plant that employs the MC process technology are primarily associated with the fuel combustion for steam production by on-site boilers, accounting for nearly $85 \%$ of the total $\mathrm{CO}_{2}$ emissions. The hydrogenation section alone accounts for approximately $57 \%$ of the total on site $\mathrm{CO}_{2}$ emissions, primarily due to the large energy input for the hydrogenation reactor. The other $\mathrm{CO}_{2}$ emission source is the solvent burning in the oxidation reactor and the combustion reactor for VOCs treatment. For the gate-togate $\mathrm{CO}_{2}$ emissions in the CEBC spray process, the fuel combustion for energy input contributes to approximately $80 \%$ of the total on site $\mathrm{CO}_{2}$ emissions in each of the four cases. The global warming potential in CEBC spray process Case 4 (similar acetic acid throughput to the MC process) is only approximately $23 \%$ of that in the MC process. In contrast, the global warming potential in the CEBC spray process that uses tenfold more acetic acid than the MC process (Case 1) is still approximately $91 \%$ of that for the MC process. The environmental benefits of eliminating the hydrogenation step in the spray process is partially offset by higher acetic acid usage, which leads to higher energy requirements for the separation columns as well as the larger off-gas $\mathrm{CO}_{2}$ emissions from solvent burning and the VOCs treatment reactor.

The conventional MC process cradle-to-gate global warming potential is about fourfold greater than that for gate-to-gate analysis (Figure 5.10), due primarily to $\mathrm{CO}_{2}$ emissions from fossil fuel-based energy required for $p \mathrm{X}$ production. Interestingly, the impact associated with $\mathrm{H}_{2}$ production from natural gas is minor because very small amounts of $\mathrm{H}_{2}$ are used for the 
hydrogenation reaction. Since similar amounts of $p \mathrm{X}$ are employed in the spray process, the increased emissions for cradle-to-gate LCA compared to gate-to-gate LCA in the spray process are about the same as those in the MC process.

\section{Acidification potential}

The acidification potential from on-site emissions is attributed to $\mathrm{SO}_{\mathrm{x}}$ and $\mathrm{NO}_{\mathrm{x}}$ emissions from the natural gas-based energy production (Figure 5.9). The gate-to-gate acidification potential correlates with the steam usage in the process. When cradle-to-gate assessment is considered, the acidification potential results primarily from the coal-based electrical power generation for producing $p \mathrm{X}$ and other raw materials. Compared to the MC process, the on-site acidification potential for case 1 (tenfold more acetic acid than the MC process) and case 4 (same acetic acid usage as the MC process) of the spray process are approximately $88 \%$ and $19 \%$, respectively, while the cradle-to-gate impacts for the two cases are approximately $96 \%$ and $85 \%$, respectively. These results show that while acetic acid usage dictate the acidification potential from on-site emissions, coal-based power generation is a major contributor to the overall acidification potential stemming from cradle-to-gate emissions.

\section{Human health non-cancer air potential}

Heavy metals (arsenic, cadmium, lead, mercury, etc.) and halogenated organic substances emitted during the fossil fuel-based energy generation (electricity and steam) contribute to this category. When compared to the MC process, the human health non-cancer air potentials of the spray process (case 1 and case 4 ) are approximately $88 \%$ and $19 \%$, respectively, based on on-site emissions, and, $94 \%$ and $69 \%$, respectively, when considering cradle-to-gate LCA. These comparisons again show the dominant effect of increased acetic acid usage on site and the use of coal-based power generation for producing the raw materials. 


\section{Ecotoxicity air potential}

Fugitive VOC emissions and VOCs emitted from various unit operations contribute primarily to the ecotoxicity air potential. The emitted VOCs associated with the TPA processes are mainly acetic acid, methyl acetate and methanol. Partitioning of inorganic chemicals into the air phase during fossil-fuel based energy generation has a relatively minor contribution compared to VOC emissions. As can be seen from Figure 5.9, for the MC process, the ecotoxicity air potential based on on-site emissions mainly stems from the fugitive acetic acid emissions from the oxidation section. The impact from the hydrogenation section, in which the relevant VOCs are mainly methanol, is relatively insignificant compared to the oxidation section (Appendix E1). There is a reflection of the fact that the solvent used in the hydrogenation process is water. The ecotoxicity air potential of the spray process that uses tenfold greater acetic acid (case 1) is greater (by approximately four times) based on both gate-to-gate and cradle-to-gate LCA results. These results show that the extent of acetic acid usage is by far the major contributor to the air toxicity potential in both processes.

\section{Smog air potential}

Smog air potential is attributed to chemicals released to air that cause smog via photochemical reactions [155]. Both the VOC emissions during the on-site TPA processes and the inorganic chemical emissions $\left(\mathrm{NO}_{\mathrm{x}}\right)$ to air during the fossil-fuel based energy generation are primary contributors to this type of air pollution. The smog air potential of the spray process case 1 is approximately 2-fold greater than that in the MC process based on on-site emissions and 1.2 times based on cradle-to-gate LCA. This trend is consistent with the greater VOC emission potential associated with acetic acid usage (see previous section). 


\section{Ecotoxicity water potential}

Organic solvents such as acetic acid, methanol and methyl acetate, as well as trace amounts of benzoic acid contained in the wastewater released to fresh water, are the primary causes of water contamination. Partitioning of metal emissions (mercury, lead, chromium, etc.) and inorganic chemicals into the water phase during the fossil-fuel based energy generation has a relatively small impact on the ecotoxicity water potential compared with the emissions/disposal of process wastewater. In the conventional MC process, the wastewater release occurs in the hydrogenation section, in which a solvent evaporator is used to concentrate the mother liquor from a centrifuge and the heavies are recycled back to the oxidation reactor while part of the lights containing trace amounts of acetic acid, methanol and benzoic acid are emitted to the water phase as process wastewater. The overhead water phase from the distillation column in the oxidation section is sent to the hydrogenation reactor for use as solvent. In the spray process, however, since the hydrogenation section is eliminated, the corresponding aqueous phase from the distillation column is released as wastewater directly, resulting in significant discharge of acetic acid, methanol and methyl acetate into the wastewater stream. This explains why the ecotoxicity water potential of the spray process is dramatically higher than that of the MC process in either gate-togate or cradle-to-gate LCA (Figures $5.9 \& 5.10$ ).

\section{Potential opportunities for improvements in the CEBC spray process}

\section{Substrate concentration (acetic acid throughput)}

The use of lower substrate $(p \mathrm{X})$ concentration in the CEBC spray process necessitates higher acetic acid throughput (e.g., nearly 10 times of acetic acid amounts in case 1 compared to the MC process). This is definitely undesirable, from both economic and environmental points of view. The higher acetic acid throughput requires bigger equipment, higher energy input, higher 
$\mathrm{CO}_{2}$ emission rates (the base number is huge despite of a lower solvent burning rate), higher makeup acetic acid amount and higher VOC emissions. The benefits of eliminating the purification step are either partially or almost totally offset (as in case 1) with respect to capital investment, operating costs and almost all the potential environmental impacts except ecotoxicity water potential, which is primarily caused by the directly released wastewater from the distillation column. To gain benefits in both the economic a well as environmental factors, as high a $p \mathrm{X}$ concentration as practically possible should be used for the alternative spray processes. The CEBC spray process would provide significant improvements in economic and environmental performances if it is able to employ the same $p \mathrm{X}$ concentration as the conventional MC process and produce polymer-grade TPA product in one step. This analysis also shows that the conventional MC process itself can be improved with respect to both economics and environmental performance if the acetic acid amount can be reduced at constant production capacity.

\section{Wastewater treatment}

Instead of directly releasing the corresponding aqueous stream (containing mainly acetic acid, methanol and methyl acetate) from the distillation column to fresh water, the wastewater stream should first be treated by passing through an absorber followed by burning the off-gas containing VOCs to $\mathrm{CO}_{2}$. Adding one or two unit operations will place a small burden on the capital and operating costs but will play an important role in minimizing the environmental impact.

p-Xylene sourcing

Comparisons of the gate-to-gate LCA and the cradle-to-gate LCA (Figures $5.9 \& 5.10$ ) indicate that the major contributors to the overall environmental impact potential stem from the fossil fuel-based energy for raw material production (primarily for $p$-xylene). Being able to use 
bio-sourced $p$-xylene as renewable feedstock instead of $p \mathrm{X}$ sourced from petroleum reformates will reduce the adverse environmental impacts, not only for the CEBC spray process, but also for the conventional MC process.

\subsection{Summary}

The spray process for producing high purity TPA from $p \mathrm{X}$ has the potential to provide both economic and environmental benefits when compared to the conventional MC process. Both benefits accrue mostly from the non-requirement of the hydrogenation step required in the conventional process for purifying the crude TPA. The estimated capital cost of the CEBC spray process is $20 \%$ lower than that of the conventional MC process even assuming tenfold more acetic acid is used in the spray process to achieve the TPA production rate. In contrast, when an identical amount of acetic acid is used in both processes, the capital cost in the spray process is $55 \%$ lower than the MC process. The corresponding costs for producing polymer-grade TPA are lower than the MC process by $3.5 \phi / \mathrm{lb}$ (when using tenfold greater acetic acid) and $10.5 \phi / \mathrm{lb}$ (when using an identical amount of acetic acid), respectively, in the two cases. The lower acetic acid usage decreases both equipment and energy costs.

The $\mathrm{GaBi}^{\circledR}$ software predicts cradle-to-grave environmental impacts for both processes that are, for most of the impact categories, generally an order of magnitude greater than the on-site (gate-to-gate) impacts. The environmental impacts are dominated by coal-based electricity generation required for producing the raw materials ( $p \mathrm{X}$, in particular). Even though acetic acid is the dominant component in the feed, $>97 \mathrm{wt} \%$ of the acetic acid used in the process is recycled such that the net usage of acetic acid per pound of product is much less than that of $p \mathrm{X}$. The emissions from coal-based electricity generation contribute to global warming, acidification potential and air pollution that cause adverse but non-cancerous human health hazards. With 
respect to both on-site emissions as well as cradle-to-gate emissions, the predicted environmental impacts for the spray process are lower than those of the $\mathrm{MC}$ process, even in the case where the acetic acid usage is tenfold greater compared to the MC process. However, such acetic acid usage contributes to greater fugitive $\mathrm{VOC}$ emissions that result in more air toxicity (non carcinogenic) and smog formation potential. Potential opportunities for improvements in the CEBC spray process are to lower the acetic acid throughput at fixed TPA production rate and to treat process wastewater before discharging to fresh water. The use of bio-sourced $p$-xylene as feedstock will improve the environmental performance of both the conventional and spray processes. 


\section{Chapter 6 Conclusions and Recommendations}

\subsection{Conclusions}

This dissertation research presents a comprehensive investigation of the oxidation of $p$-xylene to terephthalic acid in a spray reactor process. The design and development of an alternative process to the conventional Mid-Century (MC) process for terephthalic acid production that admits the principles of green chemistry and green engineering motivated this research work. Several aspects of terepthalic acid synthesis in a spray reactor were addressed, including: the acquisition of kinetic data under homogenous reaction conditions avoiding TPA precipitation; theoretical analyses and experimental demonstration of one step high-purity terephthalic acid production in a semi-batch spray reactor; rational design and preliminary demonstration of a continuous spray reactor process; and the economic and environmental impact assessments of a simulated spray process for terephthalic acid production.

Intrinsic kinetics of $p \mathrm{X}$ liquid phase oxidation to TPA was investigated in a stirred reactor under homogeneous conditions without TPA precipitation by using low $p$-xylene concentrations to provide guidelines for the rational design and optimization of the spray process for TPA production as well as reactor modeling studies. A first order kinetic model was developed and kinetic rate constants obtained based on the experimental data. The estimated rate constants are shown to be at least one order of magnitude greater than those literature reported data, probably because the reaction was operated under homogeneous conditions avoiding solid formation in the liquid phase and thereby eliminating gas-solid and liquid-solid mass transfer limitations. Our 
investigations concur with previous reports that the slowest step is the oxidation of $p$-toluic acid to 4-carboxybezaldehyde. However, our investigations show that it is not possible to completely eliminate the gas-liquid mass transfer limitations associated with the fast intermediate oxidation steps in a conventional stirred reactor, even when operating under homogeneous conditions at high rpm. This confirms that other reactor configurations (such as spray reactor) are needed to overcome gas-liquid mass transfer limitations. The effects of bromide and initial $p$-xylene concentrations on the reaction rate were also investigated. Bromide concentration shows zero order dependence at high concentrations where the intermediate 4-bromomethyl benzoic acid elimination is favored. Decreasing reaction rates with an increase of initial $p$-xylene concentration shown in the traditional three-phase reaction system were not observed in the homogeneous system (wherein the intrinsic rate constants were invariant within the respective confidence intervals), which further confirms that the solid phase hinders the reaction rate and the literature reported kinetic rate constants are not intrinsic kinetic parameters.

The spray reactor is configured to disperse the liquid phase containing the dissolved $p$-xylene and $\mathrm{Co} / \mathrm{Mn} / \mathrm{Br}$ based catalyst in acetic acid as fine droplets (by means of a nozzle) into a continuous vapor phase containing the oxidant $\left(\mathrm{O}_{2}\right)$. Each of these droplets is envisioned to function as a microreactor. The switching dispersed and continuous phases in the conventional MC process [in which the gas phase (air) is dispersed into the continuous liquid phase] was aimed at maximizing the gas/liquid interfacial mass transfer area and eliminating $\mathrm{O}_{2}$ starvation in the liquid phase, thereby avoiding incomplete oxidation to achieve improved TPA selectivity and purity, as well as reducing the solvent burning by shortening the reactor residence times.

A spray process concept for terephthalic acid production was successfully demonstrated in a $700 \mathrm{~mL}$ semi-batch spray reactor, custom designed and built in house at CEBC, in which high- 
purity terephthalic acid $\left(<25 \mathrm{ppm}\right.$ 4-carboxybenzaldehyde) was produced in one step at $200{ }^{\circ} \mathrm{C}$ and 15 bar pressure. This result is in sharp contrast to the performance of the conventional stirred reactor in which the solid terephthalic acid product contains $>1000 \mathrm{ppm}$ 4-carboxybenzaldehyde requiring further purification steps. We attribute the superior performance of the spray reactor to our initial hypothesis that the increased gas-liquid mass transfer area avoids $\mathrm{O}_{2}$ starvation that can occur when gas is sparged into a continuous liquid phase, as in the stirred reactor. In addition, the reduced backmixing in the spray reactor has also contributed to enhanced conversion. Theoretical calculations confirm that in typical spray droplets (50 $\mu$ m diameter), the $\mathrm{O}_{2}$ diffusion time constants are one to two orders of magnitude lower than the kinetic time constant (associated with the rate determining step) confirming the complete penetration of the $\mathrm{O}_{2}$ throughout the liquid phase and the elimination of $\mathrm{O}_{2}$ starvation.

Mathematical modeling of a stirred reactor operated at typical MC process conditions shows that solvent evaporation is a main source of cooling that results in steady states that are openloop stable. Based on steady state heat generation and heat removal rates, the stirred reactor model accurately predicts the reactor temperature reported in conventional reactors. In addition, the modeling also shows that control of reactor operating pressure around 15 bar is critical to achieving stable operation without temperature overshoot that might lead to solvent and titanium burning in the presence of oxygen. These model predictions were verified experimentally. Spray reactor operation in which the reactor pressure is maintained constant results in constant pressure and temperatures whereas operation in which the reactor pressure is allowed to rise during continuous gas introduction results in a steadily rising pressure and temperature in the reactor.

Gas phase concentration measurements show that $\mathrm{CO}$ formation (an indicator of solvent burning reaction) in the spray reactor is roughly one-fourth of that formed in the MC process. 
This suggests that the shorter residence times in the spray reactor could also reduce solvent burning. Further, the use of $\mathrm{CO}_{2}$ as an inert gas has the advantage of higher heat capacity and superior flame inhibition properties compared to nitrogen. The presence of $\mathrm{CO}_{2}$ and acetic acid vapor in the gas phase in sufficiently high concentrations is shown to maintain the vapor phase outside the flammability envelope.

The promising results from the semi-batch spray reactor led us to the more challenging task of demonstrating continuous spray reactor operation and its benefits. Preliminary experimental results from a larger $4 \mathrm{~L}$ continuous spray reactor during a 25 -min experimental run using a manually controlled micro-metering valve show high concentrations of intermediates. This is attributed to various factors such unsteady state operation with varying liquid holdup in the reactor, low catalyst concentration and inadequate residence time in the liquid phase. A steppermotor-controlled micro-metering valve was successfully designed and implemented in the $4 \mathrm{~L}$ continuous spray reactor. Excellent T \& P control as well as constant acetic acid holdup in the reactor were demonstrated during a 30-min blank run. Further process optimization, guided by modeling of the spray reactor, is essential to rationally address the challenging task of demonstrating continuous/stable spray reactor operation that produces high-purity TPA as observed in the semi-batch spray reactor.

Comparative economic analyses of the conventional MC process and CEBC spray process (four alternative spray cases using varying amount of acetic acid in the feed) for terephthalic acid production were performed assuming a production rate of 500,000 t/yr of purified terephthalic acid. The estimated capital cost of the CEBC spray process is $20 \%$ lower than that of the conventional MC process even though tenfold more acetic acid is used in the spray process to achieve the TPA production rate. In contrast, when an identical amount of acetic acid is fed in 
both processes, the capital cost in the spray process is $55 \%$ lower than the MC process. The corresponding costs for producing polymer-grade TPA are lower than the MC process by 3.5 $\notin / \mathrm{lb}$ (when using tenfold greater acetic acid) and $10.5 \not / l b$ (when using an identical amount of acetic acid), respectively, in the two cases. The economic benefits of the CEBC spray process are mainly derived from the non-requirement of the hydrogenation step required in the conventional process for purifying the crude TPA.

Gate-to-gate and cradle-to-gate life cycle assessments of the conventional MC process and CEBC spray process for terephthalic acid production were performed and potential environmental impacts were estimated based on the TRACI model incorporated in $\mathrm{GaBi}^{\circledR}$ software. The predicted cradle-to-grave environmental impacts for both processes are, for most of the impact categories, generally an order of magnitude greater than the on-site (gate-to-gate) impacts. Cradle-to-gate LCA reveals that the overall environmental impacts in both processes are dominated by coal-based electricity generation required for producing the raw materials $(p$ xylene, in particular). Even though acetic acid is the dominant component in the feed, $>97 \mathrm{wt} \%$ of the acetic acid used in the process is recycled such that the net usage of acetic acid per pound of product is much less than that of $p$-xylene. The emissions from coal-based electricity generation contribute to global warming, acidification potential and air pollution that cause adverse but non-cancerous human health hazards. With respect to both on-site emissions as well as cradle-to-gate emissions, the predicted environmental impacts for the spray process are lower than those of the MC process, even in the case where the acetic acid usage is tenfold greater compared to the $\mathrm{MC}$ process. However, such acetic acid usage contributes to greater fugitive VOC emissions that result in more air toxicity (non carcinogenic) and smog formation potential. Potential opportunities for improvements in the CEBC spray process are to lower the acetic acid 
throughput at fixed TPA production rate and to treat process wastewater before discharging to fresh water. The use of bio-sourced $p$-xylene as feedstock will improve the environmental performance of both the conventional and spray processes.

In summary, the spray concept for $p$-xylene oxidation to terephthalic acid has the potential to be an alternative greener process concept for making polymer-grade dicarboxylic acids in one step. The several potential green attributes (no need for hydrogenation step, reduced solvent burning, and reduced capital and operating costs as well as environmental burdens) make this process especially eminent for making chemical intermediates for renewable plastics. This spray concept could also be applied to other fast gas-liquid reaction systems.

\subsection{Recommendations}

The following studies are recommended as extensions of the research project on the spray process for terephthalic acid production.

1. This work successfully demonstrated the spray process concept to produce high-purity terephthalic acid in one step in a $700 \mathrm{~mL}$ semi-batch spray reactor (with continuous gas phase flow but the liquid phase remained in the reactor) for a 3-min duration. Even though significant efforts were made to scale up the spray reactor from $700 \mathrm{~mL}$ to $4 \mathrm{~L}$ and to make the process continuous, the demonstration of the continuous spray process under steady state operation for a longer duration (at least several hours) turned out to be more challenging than expected and has not yet been achieved. The major roadblock to the steady state continuous operation at a suitable large scale is the automatic control of the liquid effluent flow rate to achieve constant liquid holdup in the reactor. This has been partially achieved in this work with a stepper-motor driven micro-metering valve but a robust, corrosion resistant valve needs to be demonstrated for extended operation lasting days. In addition, solubility studies show that terephthalic acid has 
low solubility in acetic acid (1.6 g terephthalic acid/100 g acetic acid at $200{ }^{\circ} \mathrm{C}$ and $15 \mathrm{bar}$ ), resulting in product precipitation at reactor conditions. Hence, future reactor design should take into account the challenge of continuously flowing solids-containing liquid slurry across the valve and tubing without plugging. Lessons from high-pressure industrial polymerization reactors provide guidance for overcoming this problem [169].

2. One of the important advantages of the spray process is to reduce the solvent (acetic acid) burning due to the shorter residence times as we claimed. The solvent burning rate has not been fully investigated in this work and only $\mathrm{CO}$ formation was measured from the $700 \mathrm{~mL}$ semibatch spray reactor during a 3-min spray duration. Once the $4 \mathrm{~L}$ spray reactor setup for steady state continuous operation is ready, the quantification of $\mathrm{CO} / \mathrm{CO}_{2}$ formation should be implemented. Isotope-labeled studies with mass spectroscopy could help distinguish the $\mathrm{CO}_{2}$ formation from the burning side reactions against the $\mathrm{CO}_{2}$ used as the inert gas for the reaction system [3].

3. As the scalability and the steady state continuous operation are demonstrated and more data from the continuous runs become available, the comparative economic and environmental impact assessments of the spray process for terephthalic acid production should be continually updated to evaluate the economic viability and the environmental benefits as well as to guide process development. In addition, the plant-scale simulation of the CEBC spray process used air for the oxidant source, which was not the case in our laboratory experiments in which $\mathrm{CO}_{2} / \mathrm{O}_{2}$ was used instead of air. It is strongly recommended to investigate the $p$-xylene oxidation with air as the $\mathrm{O}_{2}$ source in the spray reactor and update the economic and environmental analyses based on these experimental data. Additionally, the analyses based on the process simulation using $\mathrm{CO}_{2}$ as the inert gas should also be performed for comparison. 
4. Modeling of the spray process for terephthalic acid production is essential for rational optimization and scale-up of the continuous process. The initial model could focus on representing the spray reactor as a series of mixed cells in the vapor phase followed by the wellmixed liquid phase at the bottom, incorporating the kinetics obtained in this work. Such a model should clearly bring out the importance of back-mixing and liquid phase residence times on the observed product purity profiles, and could provide guidance for the development of a more comprehensive model that incorporates nozzle geometry, spray pattern and detailed fluid dynamics in addition to the kinetics of the reaction system. The availability of advanced computational fluid dynamics (CFD) software, such as COMSOL Multiphysics ${ }^{\circledR}$ [170] software, makes it easier to perform such detailed reactor simulations. 


\section{Nomenclature}

$\begin{array}{ll}C_{p, j} & \text { heat capacity of species } \mathrm{j}, \mathrm{kJ} /\left(\mathrm{mol}{ }^{\circ} \mathrm{C}\right) \\ D & \text { diffusivity of } \mathrm{O}_{2} \text { in the acetic acid-dominated reaction mixture, } \mathrm{cm}^{2} / \mathrm{s} \\ F_{j 0} & \text { molar feed rate for species } \mathrm{j}, \mathrm{mol} / \mathrm{h} \\ F_{j v a p} & \text { evaporative molar rate for species } \mathrm{j}, \mathrm{mol} / \mathrm{h} \\ H_{j 0} & \text { partial molar enthalpy for species } \mathrm{j} \text { in feed stream, } \mathrm{kJ} / \mathrm{mol} \\ H_{j} & \text { partial molar enthalpy for species } \mathrm{j} \text { in effluent stream, } \mathrm{kJ} / \mathrm{mol} \\ \Delta H_{R} & \text { heat of reaction, } \mathrm{kJ} / \mathrm{mol} \\ \Delta H_{v a p, j} & \text { heat of vaporization for species } \mathrm{j}, \mathrm{kJ} / \mathrm{mol} \\ k & \text { kinetic rate constant for the rate-determining step, } \mathrm{s}^{-1} \\ N_{j} & \text { moles of species } \mathrm{j} \text { inside reactor at any instant of time, mol } \\ Q_{G} & \text { temperature of reaction mixture or effluent stream, }{ }^{\circ} \mathrm{C} \\ Q_{R} & \text { heat generation rate, } \mathrm{kJ} / \mathrm{h} \\ R_{\mathrm{p}} & \text { heat removal rate, } \mathrm{kJ} / \mathrm{h}\end{array}$




\section{Bibliography}

[1] Chemistry Explained: Green Chemistry, 2013. (http://www.chemistryexplained.com/Ge-Hy/Green-Chemistry.html, accessed May 2013)

[2] Anastas, P. T., Warner, J. C., 1998. Green Chemistry: Theory and Practice. Oxford University Press: Oxford, UK.

[3] Zuo, X., Niu, F., Snavely, K., Subramaniam, B., Busch, D.H., 2010. Liquid phase oxidation of $p$-xylene to terephthalic acid at medium-high temperatures: multiple benefits of $\mathrm{CO}_{2}$ expanded liquids. Green Chemistry 12, 260-267.

[4] Kwak, J. W., Lee, J. S., Lee, K. H., 2009. Co-oxidation of p-xylene and p-toluic acid to terephthalic acid in water solvent: kinetics and additive effects. Applied Catalysis A: General 358, 54-58.

[5] ICIS.com, 2010. (http://www.icis.com/Articles/2007/11/06/9076461/purified-terephthalic-acid-pta-usesand-market-data.html, accessed Feb. 2013)

[6] Rajagopalan, B., 2007. Investigation of dense carbon dioxide as a solvent medium for the catalytic oxidation of hydrocarbons. University of Kansas, $\mathrm{PhD}$ Dissertation.

[7] Landau, R., Heights, R., Saffer, A., 1958. Process for the preparation of terephthalic acid. US 2833818 .

[8] Ure, A.M., Parker, D., 2010. Methods, processes, and system for treating and purifying crude terephthalic acid and associated process streams. WO 2010/122304 A1.

[9] Hashmi, S.M.A., Al-Luhaidan, S., 2006. Process for preparing purified terephthalic acid. EP 1671938 A1.

[10] Meyer, D. H., 1971. Fiber-grade terephthalic acid by catalytic hydrogen treatment of dissolved impure terephthalic acid. US 3584039.

[11] BP, 2011. Annual report and form 20-F 2011 (bp.com/annualreport)

[12] Lin, R., De Vreede, M., Sluijmers, J.W., De Boer, M., Woodruff, T.E., 2006. Method for oxidizing a slurry composition in a post oxidation zone in the presence of added steam. US 20060084824 A1.

[13] Weissermel, K., Arpe, H.-J., 1997. Industrial Organic Chemistry. VCH Publishers, Inc., New York.

[14] Raghavendrachar, P., Ramachandran, S., 1992. Liquid-phase catalytic oxidation of $p$ xylene. Industrial \& Engineering Chemistry Research 31, 453-462.

[15] Packer, L. G., James, D. E., 1986. Purification of terephthalic acid. US 4626598 A.

[16] Schroeder, H., 1986. Purification of terephthalic acid to relatively low levels of 4carboxybenzaldehyde and catalyst therefor. US 4629715 A. 
[17] Schroeder, H., 1989. Recovery of terephthalic acid from its recycle streams. US 4833269 A.

[18] Bartos, T. M., 2006. Process and apparatus for manufacturing pure forms of aromatic carboxylic acids. WO 2006/102336 A2.

[19] Bartos, T. M., Leung, L. K., 2006. Process and apparatus for manufacturing aromatic carboxylic acids including pure forms thereof. WO 2006/102459 A1.

[20] Abrams, K. J., 1996. Process for preparing aromatic carboxylic acids with efficient energy recovery by the oxidation of aromatic hydrocarbon feedstocks. WO 96/11899 A1.

[21] Seslar, T., 2003. Finding the $X$ factor. Frontiers, 25-29.

[22] Graham, D. A., Hamley, P. A., Ilkenhans, T., Poliakoff, M., Woodcock, D. C., 2002. Production of aromatic carboxylic acids. WO 2002006201 A1.

[23] Turner, J. A., Royall, D. J., Hugall, D. S., Jones, G. H., Woodcock, D. C., 1998. Production of terephthalic acid by liquid-phase oxidation. WO 9838150 A1.

[24] Bickham, D. R., 1999. Hydrogenation-centrifugation process for separating pure terephthalic acid from crude reaction products. WO 9908990 A1.

[25] INVISTA, 2012. INVISTA's Spring 2013 fabric concepts for denim - reinvent, renew, recover.

(http://www.invista.com/en/news/pr-invista-spring-2013-denim-fabric-concepts.html, accessed Mar. 2013)

[26] INVISTA, 2009. INVISTA acquires unique residue recovery technology for terephthalic acid industry.

(http://www2.invista.com/news_releases/2009/pr_ipt_acquisition.shtml, accessed Mar. 2013)

[27] Piras, L., Schena, S., Chiarelli, M., Soro, L., 2001. Filtration and washing process and apparatus for the recovery of purified terephthalic acid. WO 2001/049647 A1.

[28] Piras, L., Schena, S., Chiarelli, M., Soro, L., 2001. Filtration and washing process for the recovery of crude terephthalic acid using a rotary filter. WO 2001/055075 A2.

[29] Dow, 2010. Dow technology licensing - COMPRESS ${ }^{\mathrm{TM}}$ PTA technology process. (http://www.dow.com/licensing/offer/pta.htm, accessed Mar. 2013)

[30] Kiefer, J. E., Phillips, W. V., Woodruff, T. E., 1986. Purification of terephthalic acid. US 4605763 A.

[31] Johnson, G. I., Kiefer, J. E., 1984. Purification of terephthalic acid. US 4447646 A.

[32] Nakaoka, K., Miyama, Y., Matsuhisa, S., Wakamatsu, S., 1973. Preparation of terephthalic acid using paraldehyde promotor. Industrial \& Engineering Chemistry Product Research and Development 12(2), 150-155. 
[33] Nakaoka, K., Kato, T., Matsuhisa, S., 1972. Catalytic preparation of aromatic carboxylic acids. US 3636095 A.

[34] Yoshimura, T., 1969. New process can reduce terephthalic acid costs. Chemical Engineering 76(10), 78-80.

[35] Ichikawa, Y., Yamashita, G., Tokashiki, M., Yamaji, T., New oxidation process for production of terephthalic acid from $p$-xylene. Industrial and Engineering Chemistry 62(4), 38-42.

[36] Partenheimer, W., 1995. Methodology and scope of metal/bromide autoxidation of hydrocarbons. Catalysis Today 23, 69-158.

[37] Aspen HYSYS ${ }^{\circledR}, 2009$. Version 7.1, Aspen Technologies: Calgary, Alberta, Canada.

[38] Lange, N., 1999. Lange's Handbook of Chemistry, fifteenth ed. McGraw-Hill, New York.

[39] Roffia, P., Callni, P., Motta, L., 1984. Byproduct identification in the terephthalic acid production process and possible mechanisms of their formation. Industrial and Engineering Chemistry Product Research and Development 23, 629-634.

[40] Cheng, Y., Peng, G., Wang, L., Li, X., 2009. Kinetics of burning side reaction in the liquid-phase oxidation of $p$-xylene. Chinese Journal of Chemical Engineering 17(2), 181 188.

[41] Roffia, P., Calini, P., Tonti, S., 1988. Methyl acetate: by-product in the terephthalic acid production process. Mechanisms and rates of formation and decomposition in oxidation. Industrial \& Engineering Chemistry Research 27(5), 765-770.

[42] Sheehan, R. J., 2003. Terephthalic Acid, Dimethyl Terephthalate, and Isophthalic Acid. Ullmann's Encyclopedia of Industrial Chemicstry, WILEY-VCH: Weinheim. 6th Edition, volume 35, 639-651.

[43] Hunter, R. S., 1975. The Measurement of Appearance. John Wiley \& Sons, New York.

[44] Wang, Q., Cheng, Y., Wang, L., Li, X., 2007. Semicontinuous studies on the reaction mechanism and kinetics for the liquid-phase oxidation of $p$-xylene to terephthalic acid. Industrial \& Engineering Chemistry Research 46, 8980-8992.

[45] Heiba, E. I., Dessau, R. M., Koehl, W J., Jr., 1969. Oxidation by metal salts. V. Cobaltic acetate oxidation of alkylbenzenes. Journal of the American Chemical Society 91(24), 6830-6837.

[46] Partenheimer, W., 1990. A chemical model for the Amoco "MC" oxygenation process to produce terephthalic acid. Chemical Industries (Dekker) 40 (Catalysis of Organic Reactions), 321-346.

[47] Solorzano, M. Z., 2000. Modeling, dynamic behavior and control of chemical reactors with evaporative cooling. University of Wisconsin-Madison, $\mathrm{PhD}$ Dissertation. 
[48] Kingsley, J. P., Roby, A. K., 1996. Terephthalic acid production using evaporative cooling. US 5523474 A.

[49] Fukuhara, H., 1998. Process for producing aromatic carboxylic acids, such as terephthalic acid, by oxidation of alkyl aromatics using heavy metal and bromine compound catalysts. EP 818433 A2.

[50] Cheng, Y., Li, X., Wang, L., Wang, Q., 2006. Optimum Ratio of Co/Mn in the liquidphase catalytic oxidation of $p$-xylene to terephthalic acid. Industrial \& Engineering Chemistry Research 45(12), 4156-4162.

[51] Kamiya, Y., Nakajima, T., Sakoda, K., 1966. Autoxidation of $p$-xylene catalyzed with cobalt monobromide in acetic acid. Bulletin of the Chemical Society of Japan 39(10), 2211-2215.

[52] Wang, Q., Li, X., Wang, L., Cheng, Y., Xie, G., 2005. Kinetics of $p$-xylene liquid-phase catalytic oxidation to terephthalic acid. Industrial \& Engineering Chemistry Research 44, 261-266.

[53] Belmonte, F. G., Fjare, K. A., Partenheimer, W., 1992. Process and catalyst for production of benzene polycarboxylic acids by liquid-phase oxidation of polymethylbenzenes. US 5112992 A.

[54] Broeker, J. L., Partenheimer, W., Rosen, B. I., 1995. Manufacture of aromatic dicarboxylic acids using cerium to facilitate a low bromine/metal ratio in the oxidation catalyst. US 5453538 A.

[55] Partenheimer, W., 1991. Production of polycarboxylic acids with a molybdenumactivated cobalt catalyst. US $4992580 \mathrm{~A}$.

[56] Partenheimer, W., 2003. The effect of zirconium in metal/bromide catalysts on the autoxidation of $p$-xylene. Part II. Alternative metals to zirconium and the effect of zirconium on manganese(IV) dioxide formation and precipitation with pyromellitic acid. Journal of Molecular Catalysis A: Chemical 206(1-2), 131-144.

[57] Codignola, F., Moro, A., 2000. Preparation and purification of aromatic mono- and polycarboxylic acids. WO 2000063146 A1.

[58] Hronec, M., Al Ghatta, H., Ruggieri, R., 2007. Preparation of terephthalic acid with high purity. WO 2007066365 A1.

[59] Jhung, S., Park, Y., Lee., K., Yoo, J. S., Chae, J., 2001. Method of producing aromatic carboxylic acids by oxidizing alkyl aromatic hydrocarbons or partially oxidized intermediates thereof. US 6194607 B1.

[60] Inary, M., Ohkoshi, F., Zaima, F., 1997. Process for producing terephthalic acid. US 5679847 A.

[61] Fukuhara, H., Oikawa, H., 1997. Method for producing aromatic carboxylic acids. US 5663428 A. 
[62] Jhung, S., Lee, K., Park, Y., Yoo, J. S., 2001. Method of producing aromatic carboxylic acids by oxidizing alkyl aromatic compounds or partially oxidized intermediates thereof with carbon dioxide containing gas. US $6180822 \mathrm{~B} 1$.

[63] Roby, A. K., 1997. Production of terephthalic acid with excellent optical properties through the use of pure or nearly pure oxygen as the oxidant in $p$-xylene oxidation. US 5696285.

[64] Roby, A. K., Kingsley, J. P., 1997. Process for producing an aromatic carboxylic acid. EP 796837 A1.

[65] Ramachandran, R., Sethna, R. H., Tamhankar, S. S., 1997. Production of terephthalic acid. US 5693856 A.

[66] Jhung, S., Park, Y., 2001. Method of producing aromatic carboxylic acids by oxidizing alkyl aromatic compounds or partially oxidized intermediates thereof with oxygenenriched gas. US $6175038 \mathrm{~B} 1$.

[67] Edwards, C. L., June, R. L., Potter, M. W., Simpson, E. J., 2000. Method to produce aromatic dicarboxylic acids using cobalt and zirconium catalysts. US $6153790 \mathrm{~A}$.

[68] Chester, A. W., Scott, E. J. Y., Landis, P. S., 1977. Zirconium cocatalysis of the cobaltcatalyzed autoxidation of alkylaromatic hydrocarbons. Journal of Catalysis 46(3), 308319.

[69] Yen, D., 2003. Catalytic solution system for manufacturing terephthalic acid and a method for same. US 20030078451 A1.

[70] Li, K., Li, S., 2008. $\mathrm{CoBr}_{2}-\mathrm{MnBr}_{2}$ containing catalysts for catalytic oxidation of $p$-xylene to terephthalic acid. Applied Catalysis, A: General 340, 271-277.

[71] Tashiro, Y., Iwahama, T., Sakaguchi, S., Ishii, Y., 2001. A new strategy for the preparation of terephthalic acid by the aerobic oxidation of $p$-xylene using $\mathrm{N}$ hydroxyphthalimide as a catalyst. Advanced Synthesis \& Catalysis 343, 220-225.

[72] Saha, B., Koshino, N., Espenson, J. H., 2004. N-hydroxyphthalimides and metal cocatalysts for the autoxidation of $p$-xylene to terephthalic acid. Journal of Physical Chemistry 108(3), 425-431.

[73] Koshino, N., Saha, B., Espenson, J. H., 2003. Kinetic study of the phthalimide $N$-oxyl radical in acetic acid. Hydrogen abstraction from substituted toluenes, benzaldehydes, and benzyl alcohols. Journal of Organic Chemistry 68(24), 9364-9370.

[74] Cai, Y., Koshino, N., Saha, B., Espenson, J. H., 2005. Kinetics of self-decomposition and hydrogen atom transfer reactions of substituted phthalimide $N$-oxyl radicals in acetic acid. Journal of Organic Chemistry 70(1), 238-243.

[75] Graham, D. A., Hamley, P. A., Oliver, R., Poliakoff, M., Woodcock, D., Ilkenhans, K., 2004. Production of aromatic carboxylic acids. US 6765113. 
[76] Dunn, J. B., Urquhart, D. I., Savage, P. E., 2002. Terephthalic acid synthesis in supercritical water. Advanced Synthesis \& Catalysis 344, 385-392.

[77] Dunn, J. B., Savage, P. E., 2002. Terephthalic acid synthesis in high-temperature liquid water. Industrial \& Engineering Chemistry Research 41, 4460-4465.

[78] Fraga-Dubreuil, J., Poliakoff, M., 2006. Organic reactions in high-temperature and supercritical water. Pure and Applied Chemistry 78, 1971-1982.

[79] Sikkenga, D. L., Pandya, A. K., Zaenger, I. C., Abrams, K. J., Bartos, T. M., 2003. Production of high purity aromatic carboxylic acid by oxidation in benzoic acid and water solvent. US 6562997 B2.

[80] Klier, J., Tucker, C. J., Kalantar, T. H., Burdett, K. A., Green, D. P., Piras, L., Schena, S., Lee, G. J., 2004. Oxidation of alkyl aromatic compounds to aromatic acids in an aqueous medium. US 6759550 B1.

[81] Nexant, Inc., 2005. Squeezing profitability from the PTA/PET value chain: impact of the latest technology advances.

(http://www.chemsystems.com/reports/search/docs/prospectus/mc-polyester-pros.pdf, accessed Dec. 2012)

[82] Melhem, G. A., 1997. A detailed method for estimating mixture flammability limits using chemical equilibrium. Process Safety Progress 16, 203-218.

[83] Sheehan, R. J., 2003. Terephthalic acid, dimethyl terephthalate, and isophthalic acid. Ullmann's Encyclopedia of Industrial Chemicstry, WILEY-VCH: Weinheim. 6th Edition, volume 35, 639-651.

[84] Izumisawa, T., Kawahara, T., Toyosawa, A., 1996. Process for producing terephthalic acid. US 5567842 A.

[85] Wang, Q., Xu, H., Li, X., 2005. Solid-liquid equilibria of 1,4-benzenedicarboxylic acid in binary acetic acid + water solvent mixtures at elevated temperatures. Journal of Chemical and Engineering Data 50(1), 258-260.

[86] Wang, Q., Xu, H., Li, X., 2005. Solubility of terephthalic acid in aqueous acetic acid from 423.15 to 513.15K. Fluid Phase Equilibria 233(1), 81-85.

[87] Chen, M., Ma, P., 2004. Solid-liquid equilibria of several systems containing acetic acid. Journal of Chemical and Engineering Data 49(4), 756-759.

[88] Ma, P., Chen, M., 2003. Solid-liquid equilibrium of terephthalic acid in several solvents. Chinese Journal of Chemical Engineering 11(3), 334-337.

[89] Han, N., Zhu, L., Wang, L., Fu, R., 1999. Aqueous solubility of $m$-phthalic acid, $o$ phthalic acid and $p$-phthalic acid from 298 to 483 K. Separation and Purification Technology 16(2), 175-180.

[90] D'oro, P. C., Danoczy, E., Roffia, P., 1980. Low temperature oxidation of $p$-xylene. Oxidation Communications 1(2), 153-162. 
[91] Morbidelli, M., Paludetto, R., Carra, S., 1986. Gas-liquid autoxidation reactors. Chemical Engineering Science 41, 2299-2307.

[92] Jacobi, R., Baerns, M., 1983. The effect of oxygen transfer limitation at the gas-liquid interphase. Kinetics and product distribution of the $p$-xylene oxidation. Erdoel \& Kohle, Erdgas, Petrochemie 36 (7), 322-326.

[93] Sun, W., Pan, Y., Zhao, L., Zhou, X., 2008. Simplified free-radical reaction kinetics for $p$-xylene oxidation to terephthalic acid. Chemical Engineering \& Technology 31 (10), 1402-1409.

[94] Cao, G., Pisu, M., Morbidelli, M., 1994. A lumped kinetic model for liquid-phase catalytic oxidation of $p$-xylene to terephthalic acid. Chemical Engineering Science 49 (24B), 5775-5783.

[95] Cao, G., Servida, A., Pisu, M., 1994. Kinetics of $p$-xylene liquid-phase catalytic oxidation. AIChE Journal 40 (7), 1156-1166.

[96] Cincotti, A., Orrù, A., Cao, G., 1997. Effect of catalyst concentration and simulation of precipitation processes on liquid-phase catalytic oxidation of $p$-xylene to terephthalic acid. Chemical Engineering Science 52 (21/22), 4205-4213.

[97] Cincotti, A., Orrù, A., Cao, G., 1999. Kinetics and related engineering aspects of catalytic liquid-phase oxidation of $p$-xylene to terephthalic acid. Catalysis today 52, 331-347.

[98] Wang, Q., Li, X., Wang, L., Cheng, Y., Xie, G., 2005. Kinetics of $p$-xylene liquid-phase catalytic oxidation to terephthalic acid. Industrial \& Engineering Chemistry Research 44, 261-266.

[99] Yan, X., 2007. Data mining macrokinetic approach based on ANN and its application to model industrial oxidation of p-xylene to terephthalic acid. Chemical Engineering Science 62, 2641-2651.

[100] Dong, Y., Yan, X., 2013. Hybrid model of industrial $p$-xylene oxidation incorporated fractional kinetic model with intelligent models. Industrial \& Engineering Chemistry Research 52, 2537-2547.

[101] Qian, F., Tao, L., Sun, W., Du, W., 2012. Development of a free radical kinetic model for industrial oxidation of $p$-xylene based on artificial neural network and adaptive immune genetic algorithm. Industrial \& Engineering Chemistry Research 51, 3229-3237.

[102] Viola, A., Cao, G., 1996. A rapid direct analysis of $p$-xylene oxidation products by reverse phase HPLC. Journal of Chromatographic Science 34, 27-33.

[103] Athena Visual Studio, 2012. Athena Visual Studio v 14.2, Athena Visual Software, Inc.

[104] Sawada, K., Tanaka, M., 1977. Formation of bromo complexes of cobalt(II) in acetic acid. Journal of Inorganic and Nuclear Chemistry 39(2), 339-344.

[105] Metelski, P. D., Adamian, V. A., Espenson, J. H., 2000. Mechanistic role of benzylic bromides in the catalytic autoxidation of methylarenes. Inorg. Chem. 39, 2434-2439. 
[106] Partenheimer, W., 2004. The complex synergy of water in the metal/bromide autoxidation of hydrocarbons caused by benzylic bromide formation. Adv. Synth. Catal. 346, 297-306.

[107] Patenheimer, W., 2005. The complex synergy of water in the metal/bromide autoxidations. Part II. Effect of water and catalyst on the aerobic oxidation of benaldehydes and the effect of water on the elementary catalytic pathways. Adv. Synth. Catal. 347, 580-590.

[108] Saha, B., Espenson, J. H., 2007. Low-bromide containing MC catalyst for the autoxidation of para-xylene. Journal of Molecular Catalysis A: Chemical 271, 1-5.

[109] Levenspiel, O., 1999. Chemical Reaction Engineering. $3^{\text {rd }}$ ed. John Wiley \& Sons, Inc., New York.

[110] O'Meadhra, Ruairi S., Lin, R., 2007. Apparatus and process for preparation and purification of terephthalic acid by oxidative digestion with optimized agitation. US 20070155987 A1.

[111] Gnagnetti, A., Piras, L., Kar, K., 2002. Impeller agitation system for dialkylbenzene oxidation reactors used for the manufacture of benzenedicarboxylic acids. WO 2002092549 A1.

[112] Osada, M., Savage, P. E., Terephthalic acid synthesis at higher concentrations in hightemperature liquid water. 1. Effect of oxygen feed method. AIChE Journal 55(3), 710716.

[113] Cussler, E.L., 2009. Diffusion: Mass Transfer in Fluid Systems, third ed. Cambridge University Press, New York.

[114] Hilmen, E.K., 2000. Separation of azeotropic mixtures: tools for analysis and studies on batch distillation operation. Norwegian University of Science and Technology, PhD Dissertation.

[115] Carlson, E. C., 1996. Don't gamble with physical properties for simulations. Chemical Engineering Progress 92 (10), 35-46.

[116] Houndonougbo, Y., Jin, H., Rajagopalan, B., Wong, K., Kuczera, K., Subramaniam, B., Laird, B., 2006. Phase equilibria in carbon dioxide expanded solvents: experiments and molecular simulations. Journal of Physical Chemistry B 110, 13195-13202.

[117] Krag, P. W., Henson, H. R., 1993. Materials selection for sulfide pressure oxidation autoclaves. Flammability and Sensitivity of Materials in Oxygen-Enriched Atmospheres: 6th Volume ASTM STP 1197, American Society for Testing and Materials, Philadelphia.

[118] Brundtland, G. H., 1987. Our common future: report of the world commission on environment and development. United Nations.

[119] Shadiya, O. O., 2010. Social, economic and environmental metrics for the sustainable optimization of chemical and petroleum processes. Oklahoma State University, PhD Dissertation. 
[120] Adams, W. M., 2006. The future of sustainability: re-thinking environment and development in the twenty-first century. Report of the IUCN Renowned Thinkers Meeting, 29-31.

[121] ICCA, 2002. On the road to sustainability: a contribution from the global chemical industry to the world summit on sustainable development. A summary of ICCA Chemical Sector Report to UNEP.

[122] Schwarz, J., Beloff, B., Beaver, E., 2002. Use sustainability metrics to guide decisionmaking. Chemical Engineering Progress 98(7), 58-63.

[123] Dunn, J. B., Savage, P. E., 2003. Economic and environmental assessment of hightemperature water as a medium for terephthalic acid synthesis. Green Chemistry 5(5), 649-655.

[124] Dunn, J. B., Savage, P. E., 2002. Economic and environmental assessment of terephthalic acid synthesis in high-temperature water. Annual Meeting Archive - American Institute of Chemical Engineers, Indianapolis, IN, United States, 158-160.

[125] Fang, J., Jin, H., Ruddy, T., Pennybaker, K., Fahey, D., Subramaniam, B., 2007. Economic and environmental impact analyses of catalytic olefin hydroformylation in $\mathrm{CO}_{2}$-expanded Liquid (CXL) media. Industrial \& Engineering Chemistry Research 46(25), 8687-8692.

[126] Gong, K., Chafin, S., Pennybaker, K., Fahey, D., Subramaniam, B., 2008. Economic and environmental impact analyses of solid acid catalyzed isoparaffin/olefin alkylation in supercritical carbon dioxide. Industrial \& Engineering Chemistry Research 47(23), 90729080 .

[127] Ghanta, M., Ruddy, T., Fahey, D. R., Busch, D. H., Subramaniam, B., 2013. Is the liquidphase $\mathrm{H}_{2} \mathrm{O}_{2}$-based ethylene oxide process more economical and greener than the gasphase $\mathrm{O}_{2}$-based silver-catalyzed process. Industrial \& Engineering Chemistry Research $52,18-29$.

[128] Ghanta, M., Fahey, D. R., Busch, D. H., Subramaniam, B., 2013. Comparative economic and environmental assessments of $\mathrm{H}_{2} \mathrm{O}_{2}$-based and tertiary butyl hydroperoxide-based propylene oxide technologies. ACS Sustainable Chemistry \& Engineering 1(2), 268-277.

[129] Peters, M. S., Timmerhaus, K. D., West, R. E., 2004. Plant Design and Economics for Chemical Engineers. 5th ed., McGraw Hill: Boston, MA.

[130] Walas, S. M., 1988. Chemical Process Equipment: Selection and Design. Butterworth Publishers: Stoneham, MA.

[131] Banker, J. G., Forrest, A. L., 1996. Titanium/steel explosion bonded clad for autoclaves and reactors. Advances in the Science and Technology of Titanium Alloy Processing, Proceedings of an International Symposium held at the 125th TMS Annual Meeting and Exhibition, Anaheim, Calif., 549-559. 
[132] Donaldson, P. A., Milne, I. P., Hollins, A. M., Usher, G. W., Gardner, M. W., Ward, P. N., 1998. Production of aromatic carboxylic acids. WO 9818749.

[133] Hsu, Y., Lin, C., 2001. Process for producing highly pure aromatic carboxylic acids. US 6245939.

[134] Parten, W. D., Ure, A. M., 1999. Dehydration of acetic acid by azeotropic distillation in the production of an aromatic acid. US 5980696.

[135] Royall, D. J., Bartlett, J. A., Fairhurst, R. G., 2001. Production of aromatic carboxylic acids and recovery of $\mathrm{Co} / \mathrm{Mn}$ catalyst from ash. US 6255510.

[136] Turner, J. A., Jeffery, I. C., 1996. Effluent gas treatment. WO 9639595.

[137] Romanenko, A. V., Likholobov, V. A., Timofeeva, M. N., Hva, J. S., Seok, P. J., 2004. Catalytic composition, method for manufacturing thereof and method for the purification of terephthalic acid. US 6753290.

[138] CEPCI. "Chemical engineering plant cost index," Chemical Engineering 2012 Oct. 64.

[139] http://apps.catalysts.basf.com/apps/eibprices/mp/ (BASF Catalysts-Metal Prices) (accessed Feb. 2013).

[140] ICIS Pricing, personal contact.

[141] Beloff, B., Lines, M., Tanzil, D., 2005. Transforming Sustainability Strategy into Action. John Wiley \& Sons, Inc., Hoboken, New Jersey.

[142] Darton, R. C., 2003. Scenarios and metrics as guides to a sustainable future the case of energy supply. Trans IChemE Part B 81, 295-302.

[143] Burgess, A. A., Brennan, D. J., 2001. Application of life cycle assessment to chemical processes. Chemical Engineering Science 56, 2589-2604.

[144] Wikipedia, 2013. (http://en.wikipedia.org/wiki/Life-cycle_assessment, accessed March 2013)

[145] Huppes, G., 1996. LCA yesterday, today and tomorrow. Institute of Environmental Sciences (CML), Leiden University, Leiden, the Netherlands, 29-49.

[146] Udo de Haes, H. A., 1993. Applications of life cycle assessment: expectations, drawbacks and perspectives. Journal of Cleaner Production 1(3-4), 131-137.

[147] Voet, E. Van der, 1996. Substances from cradle to grave: development of a methodology for the analysis of substances flows through the economy and the environment of a region: with case studies on cadmium and nitrogen compounds. Leiden University, Leiden, the Netherlands, PhD Dissertation.

[148] ISO 14040, 2006. Environmental management — Life cycle assessment — Principles and framework, International Organization for Standardization, ISO, Geneva.

[149] ISO 14044, 2006. Environmental management - Life cycle assessment — Requirements and guidelines, International Organization for Standardization, ISO, Geneva. 
[150] Elock, D., 2007. Life-cycle thinking for the oil and gas exploration and production industry. Argonne National Laboratory, Argonne, IL.

[151] Sharaai, A. H., Mahmood, N. Z., Sulaiman, A. H., 2010. Life cycle impact assessment (LCIA) using TRACI methodology: an analysis of potential impact on potable water production. Australian Journal of Basic and Applied Sciences 4(9), 4313-4322.

[152] GaBi, 2012. Version 6. PE International, Germany.

[153] Bare, J. C., Hofstetter, P., Pennington, D. W., Udo de Haes, H. A., 2000. Life cycle impact assessment workshop summary: Midpoints versus endpoints: The sacrifices and benefits. Int. J. Life Cycle Assess. 5 (6), 319-326.

[154] Bare, J. C., 2002. Developing a consistent decision-making framework by using the U.S. EPA's TRACI. US Environmental Protection Agency, Cincinnati, OH.

[155] Bare, J. C., Norris, G. A., Pennington, D. W., McKone, T., 2002. TRACI: The tool for the reduction and assessment of chemical and other environmental impacts. Journal of Industrial Ecology 6 (3-4), 49-78.

[156] Curran, M. A., 2004. The status of life-cycle assessment as an environmental management tool. Environmental Progress 23 (4) 277-283.

[157] TRI Explorer, 2013. (http://iaspub.epa.gov/triexplorer/tri_release.chemical, accessed March 2013)

[158] GHG data publication tool, 2013. (http:/ghgdata.epa.gov/ghgp/main.do, accessed March 2013)

[159] Schaich, J. R., 1991. Estimate fugitive emissions from process equipment. Chemical Engineering Progress, 31-35.

[160] Siegell, J. H., 1997. Improve VOC emission predictions. Hydrocarbon Processing, 119121.

[161] Shonnard, D., Hiew, D., 2000. Comparative environmental assessments of VOC recovery and recycle design alternatives for a gaseous waste stream. Environmental Science and Technology 34(24), 5222-5228.

[162] Fabri, J., Graeser, U., Simo, T. A., 2003. Xylenes. Ullmann's Encyclopedia of Industrial Chemicstry, WILEY-VCH: Weinheim. 6th Edition, volume 39, 583-603.

[163] GaBi Database Content, 2013a.

(http://gabi-dataset-documentation.gabi-software.com/xml_data/processes/29b2ba8c4200-4d3c-a671-53f264b0bf2b_05.00.000.xml, accessed March 2013)

[164] Cheung, H., Tanke, R., Torrence, G. P., 2003. Acetic acid. Ullmann's Encyclopedia of Industrial Chemicstry, WILEY-VCH: Weinheim. 6th Edition, volume 1, 149-178.

[165] GaBi Database Content, 2013 b.

(http://gabi-6-lci-documentation.gabi-software.com/xml-data/processes/80c0f36b-90894058-9940-3fa073e8bfc5.xml, accessed March 2013) 
[166] GaBi Database Content, 2013c.

(http://gabi-6-lci-documentation.gabi-software.com/xml-data/processes/bdb79573-38724dc2-9bc7-41a4c624c37b.xml, accessed March 2013)

[167] GaBi Database Content, $2013 d$.

(http://gabi-6-lci-documentation.gabi-software.com/xml-data/processes/300e0734-6b744225-a078-d64108783da3.xml, accessed March 2013)

[168] Allen, D. T., Shonnard, D. R., 2002. Green Engineering: Environmentally Conscious Design of Chemical Processes. Prentice-Hall, Inc., Upper Saddle River, NJ.

[169] Burns, D. H., Hein, J. E., Rajaendran, G. K., Verser, D. W., 2011. Continuous withdrawal from high solids slurry polymerization. EP 1554317 B1.

[170] COMSOL Multiphysics, 2013. Version 4.3b, COMSOL, Inc.: Los Angeles, CA, US. 


\section{Appendices}

\section{Appendix A: Numerical Data of the Experimental Solubilities}

Table A1. Experimental solubilities of TPA, 4-CBA and $p$-TA in acetic acid or aqueous acetic acid

\begin{tabular}{|c|c|}
\hline \multicolumn{2}{|c|}{ Solubilities of TPA in acetic acid } \\
\hline $\mathrm{T}\left({ }^{\circ} \mathrm{C}\right)$ & S (g TPA/100 g HOAc) \\
\hline 118.5 & 0.243 \\
\hline 134.5 & 0.318 \\
\hline 153.1 & 0.392 \\
\hline 171.3 & 0.646 \\
\hline 180.1 & 1.061 \\
\hline 202.4 & 1.618 \\
\hline 214.5 & 2.038 \\
\hline \multicolumn{2}{|c|}{ Solubilities of TPA in $95 \mathrm{wt} \%$ aqueous acetic acid } \\
\hline $\mathrm{T}\left({ }^{\circ} \mathrm{C}\right)$ & S (g TPA/100 g solvent) \\
\hline 117.5 & 0.242 \\
\hline 137.2 & 0.389 \\
\hline 149.5 & 0.584 \\
\hline 171.2 & 0.963 \\
\hline 187 & 1.406 \\
\hline 202.7 & 1.968 \\
\hline 208.5 & 2.219 \\
\hline \multicolumn{2}{|c|}{ Solubilities of 4-CBA in acetic acid } \\
\hline $\mathrm{T}\left({ }^{\circ} \mathrm{C}\right)$ & $\mathrm{S}(\mathrm{g} 4-\mathrm{CBA} / 100 \mathrm{~g} \mathrm{HOAc})$ \\
\hline 112 & 6.238 \\
\hline 124 & 10.18 \\
\hline 148 & 23.09 \\
\hline 179 & 45.25 \\
\hline 196 & 68.71 \\
\hline \multicolumn{2}{|c|}{ Solubilities of $p$-TA in acetic acid } \\
\hline $\mathrm{T}\left({ }^{\circ} \mathrm{C}\right)$ & $\mathrm{S}(\mathrm{g} p-\mathrm{TA} / 100 \mathrm{~g} \mathrm{HOAc})$ \\
\hline 79 & 31.45 \\
\hline 97 & 63.26 \\
\hline 112 & 125.6 \\
\hline 127 & 261.7 \\
\hline
\end{tabular}




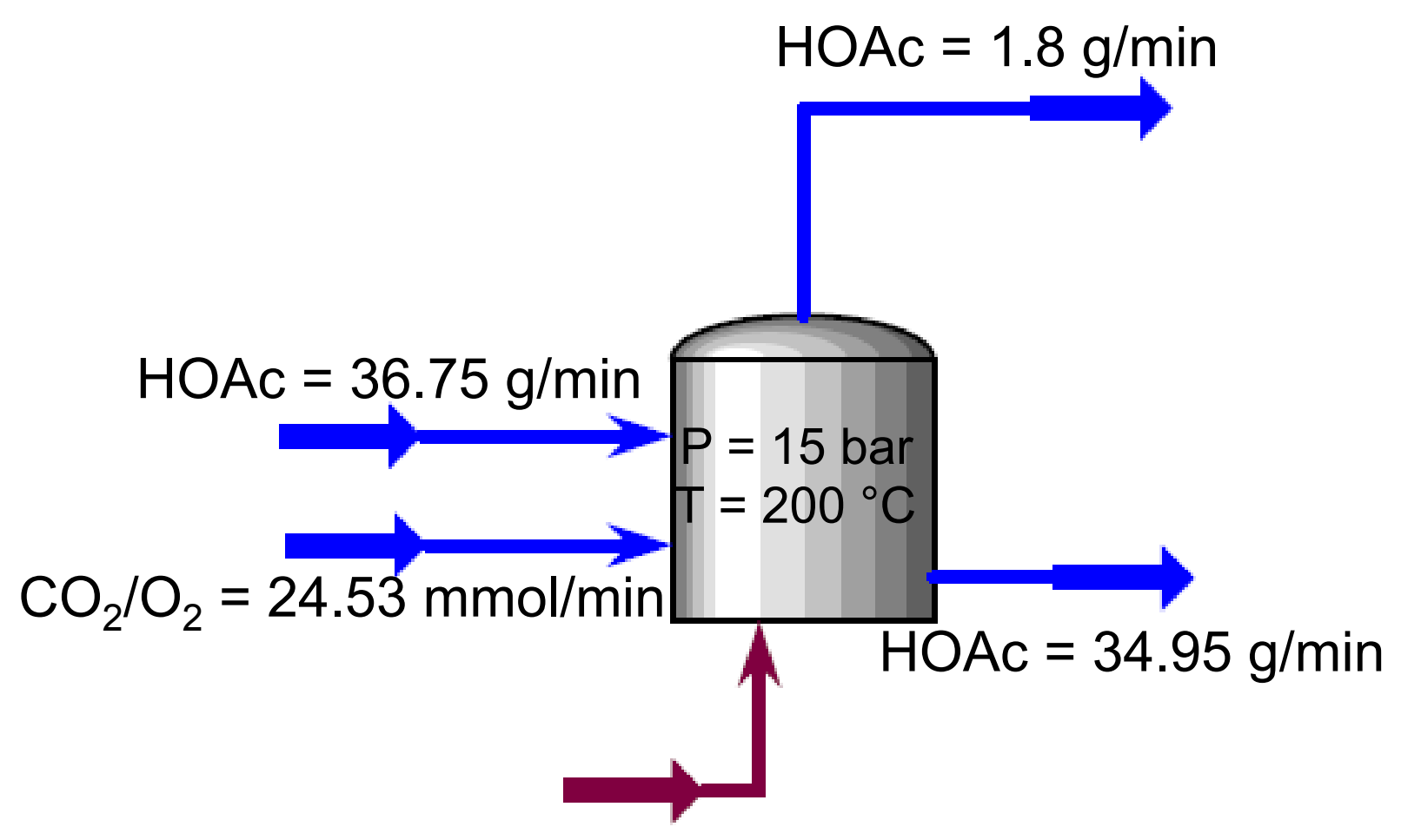

Figure B1: HYSYS simulation of saturated acetic acid flow in the vapor phase 


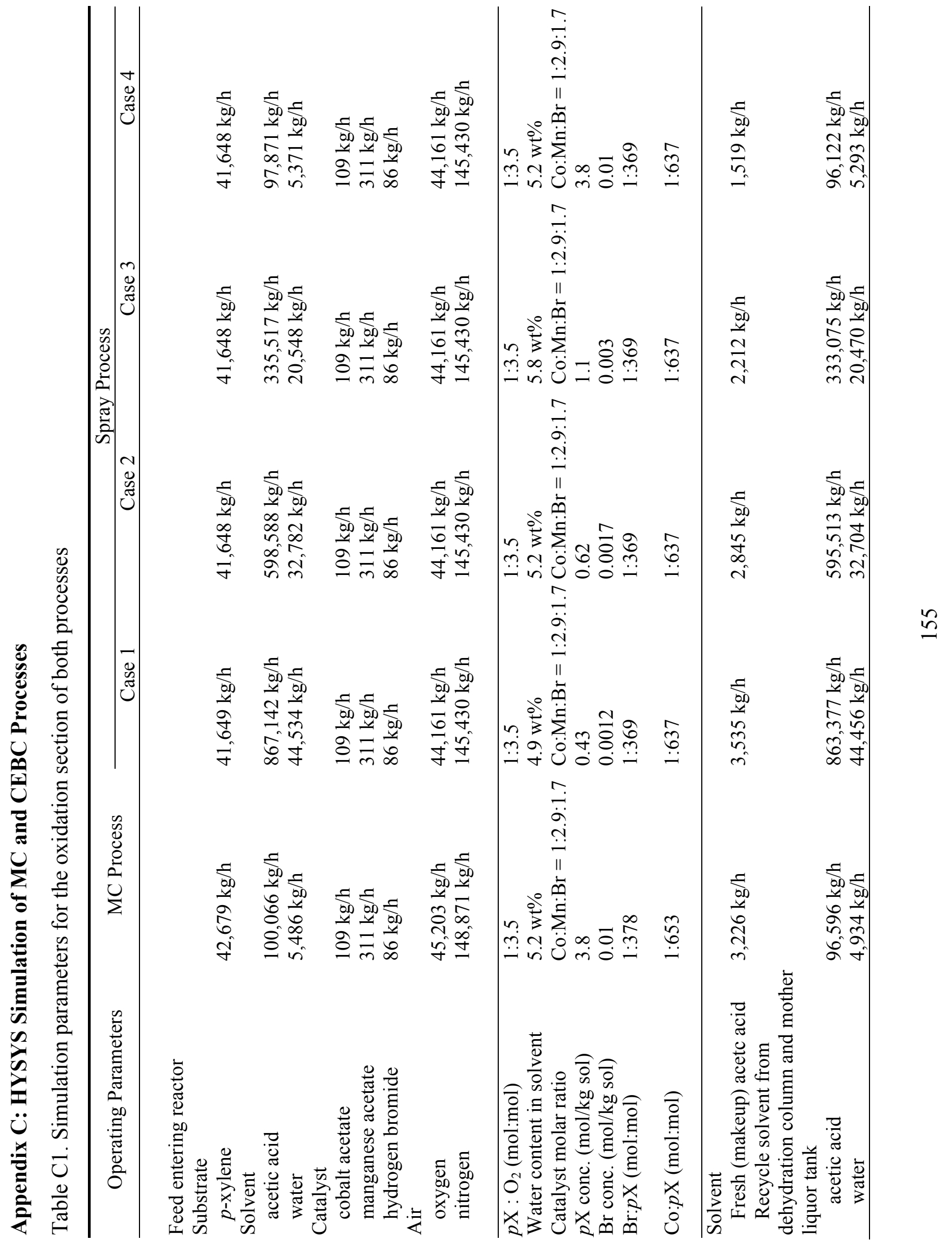




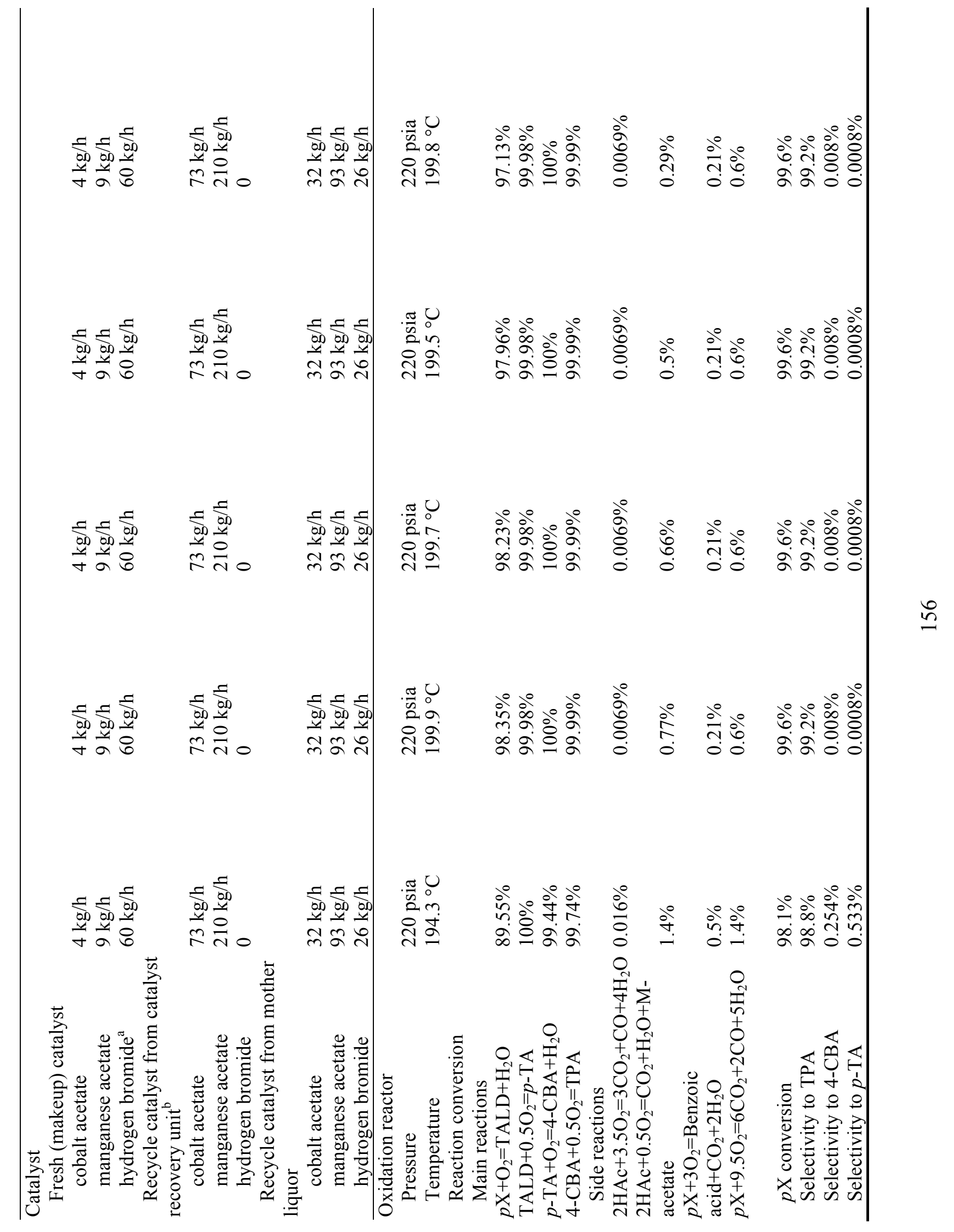




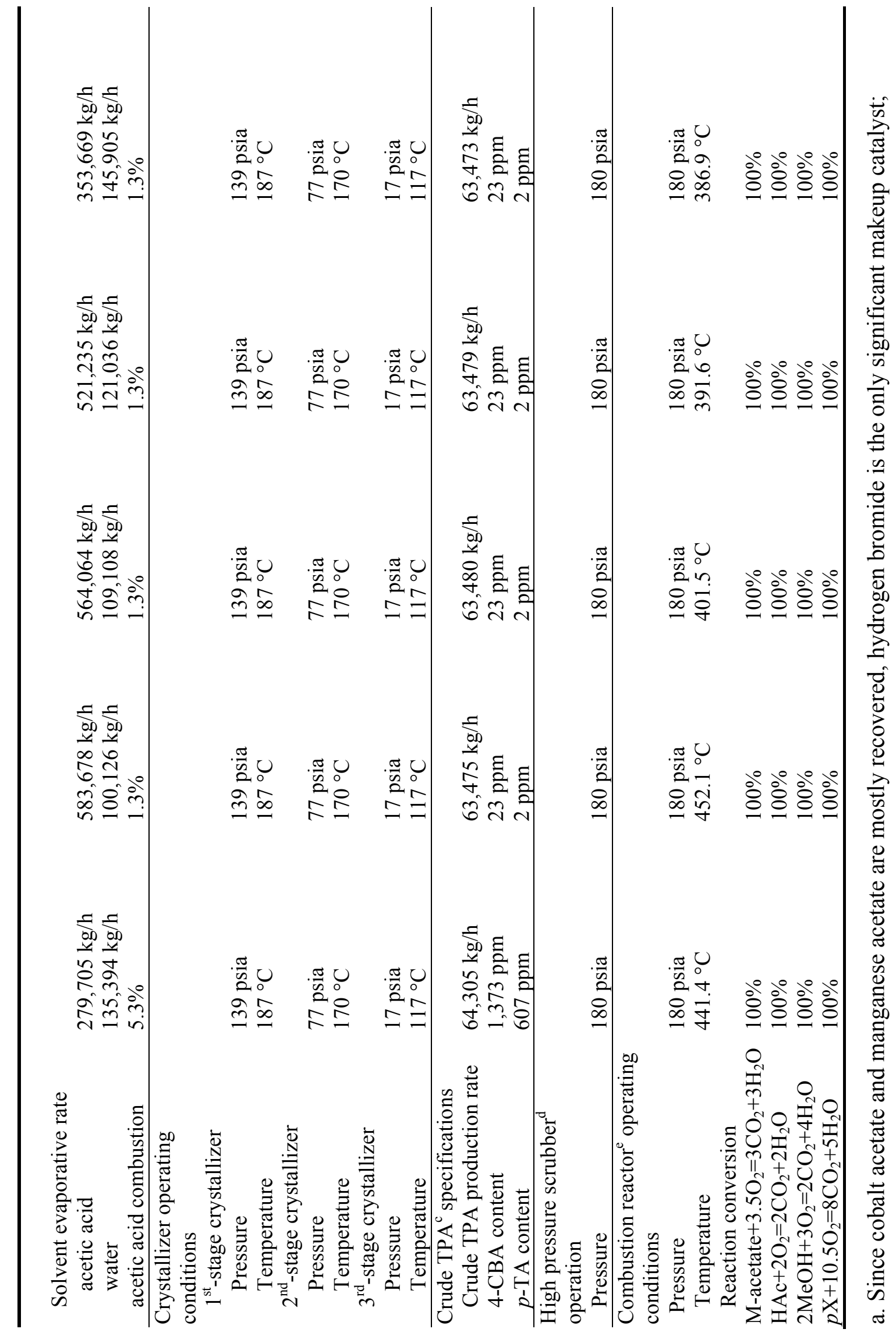




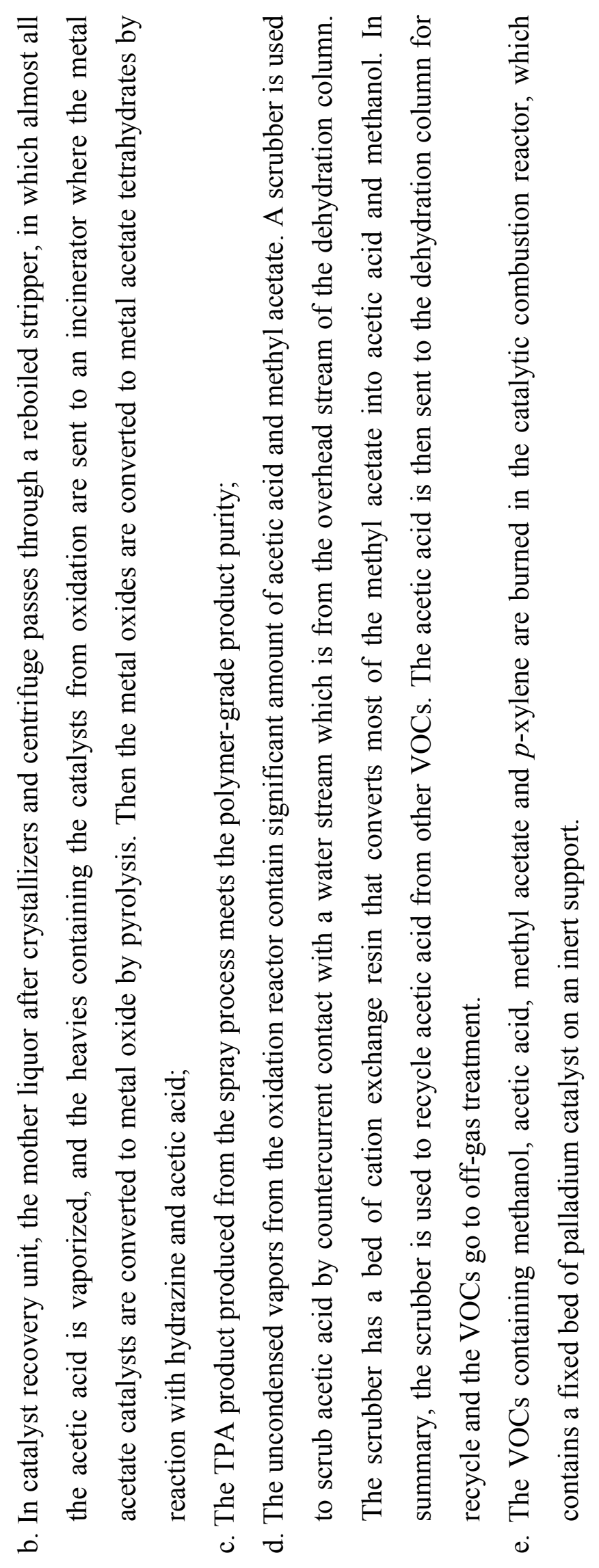




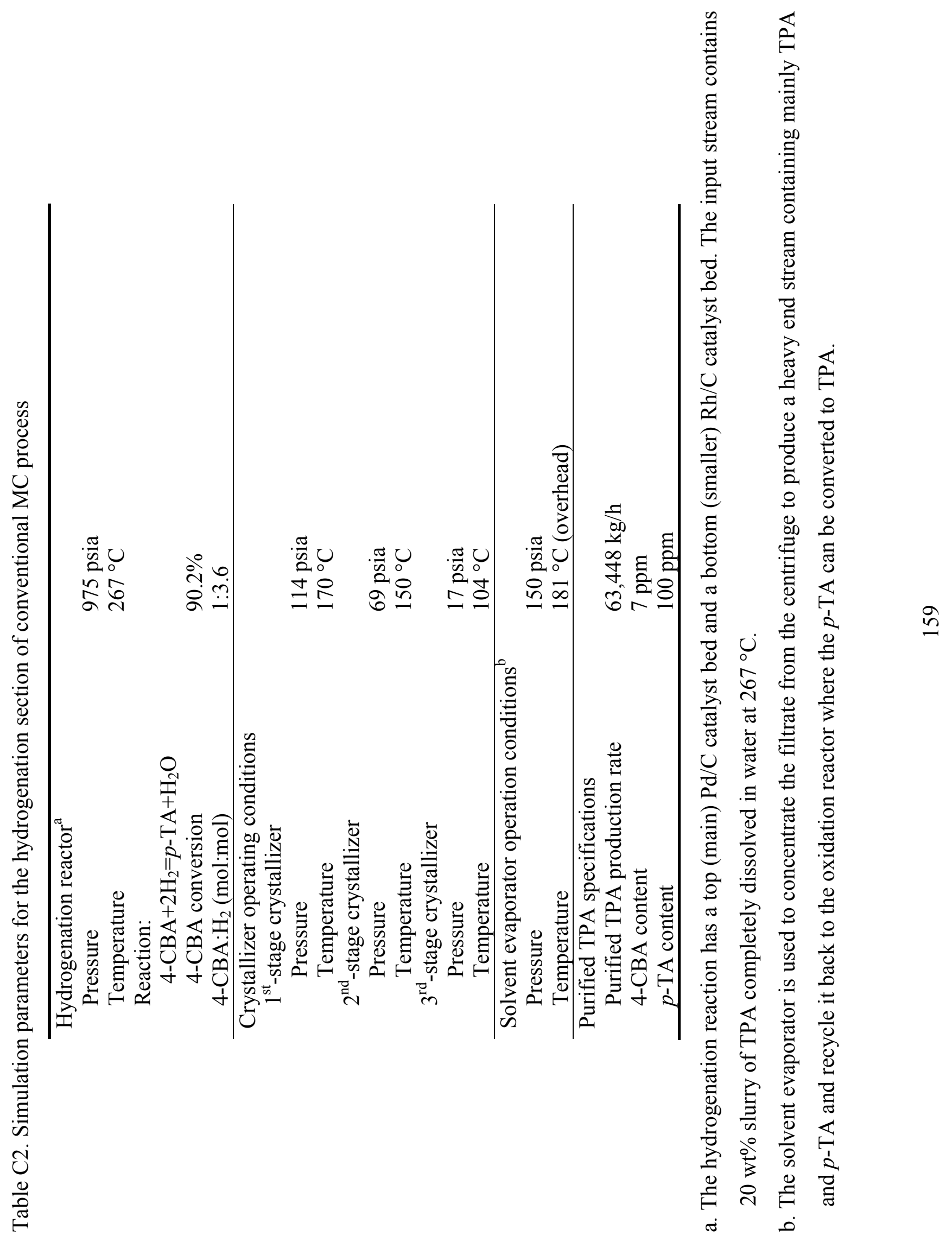




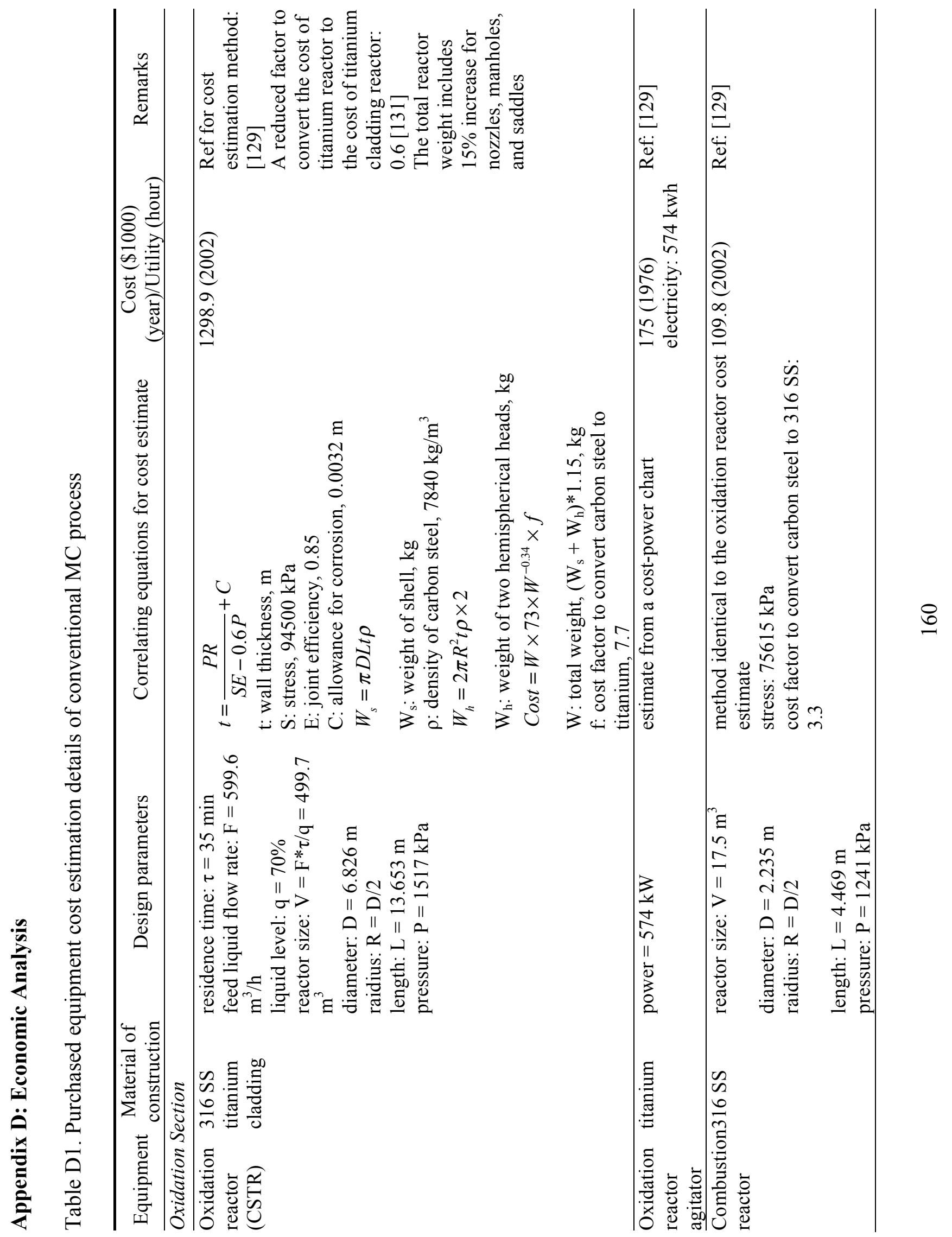




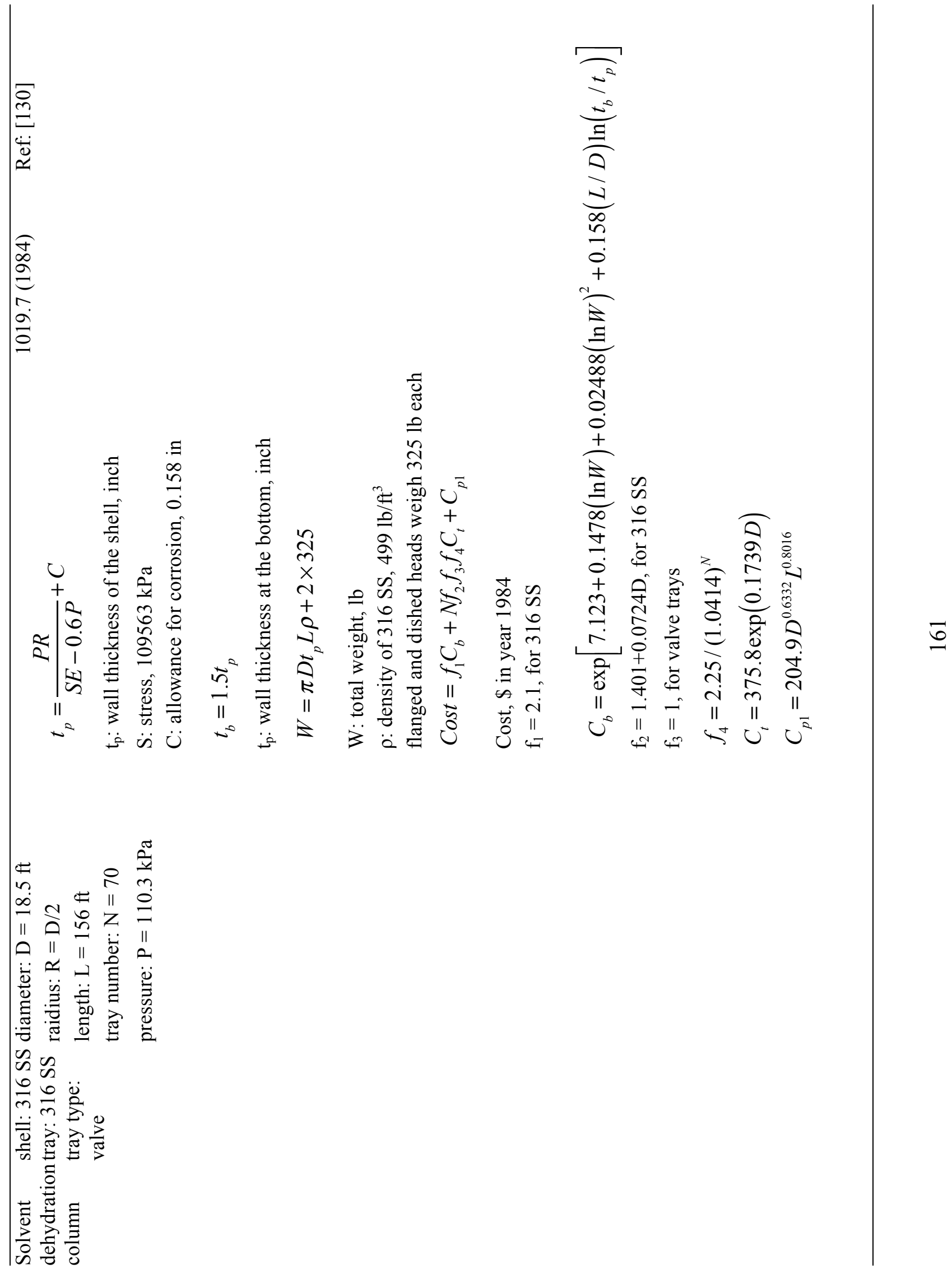



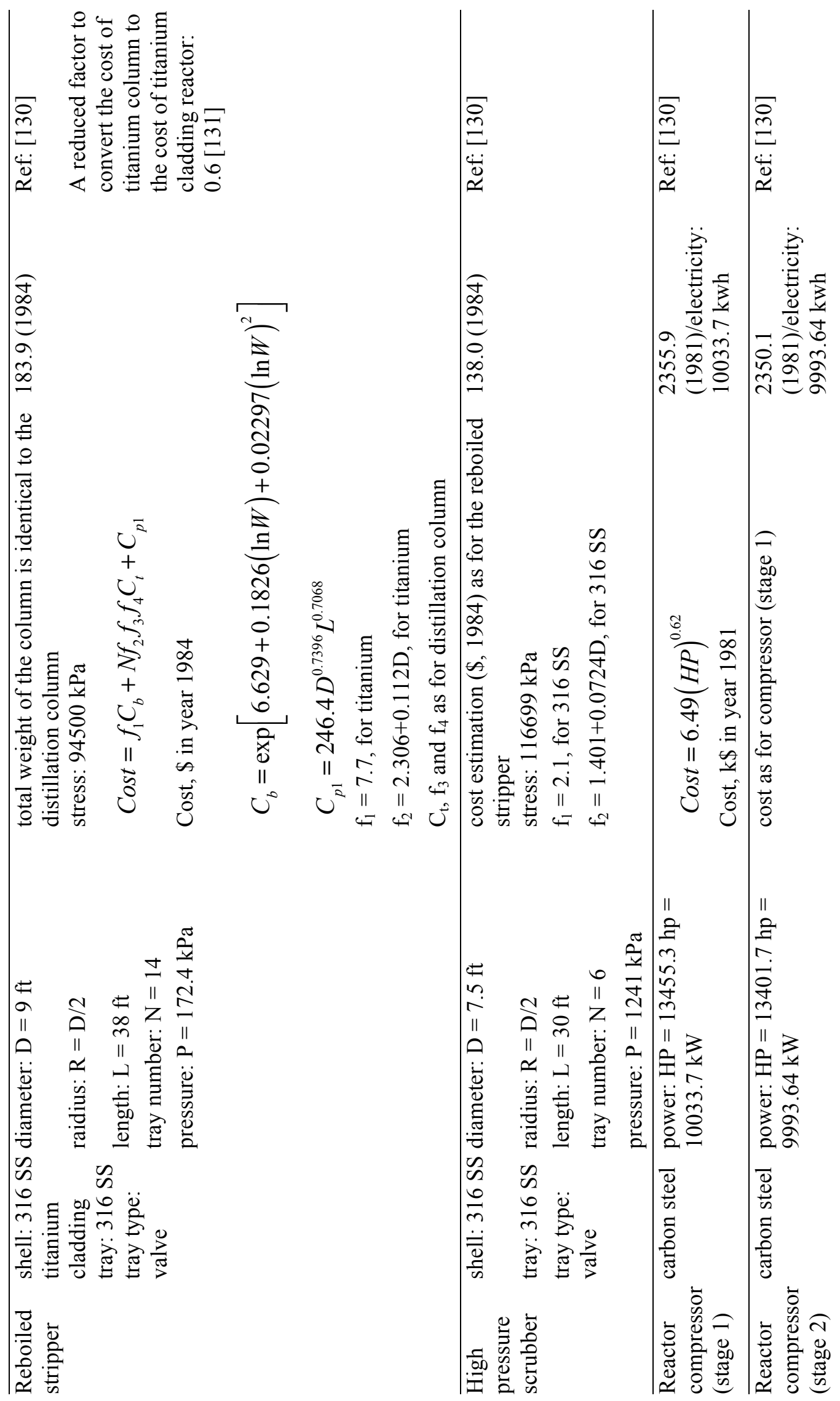


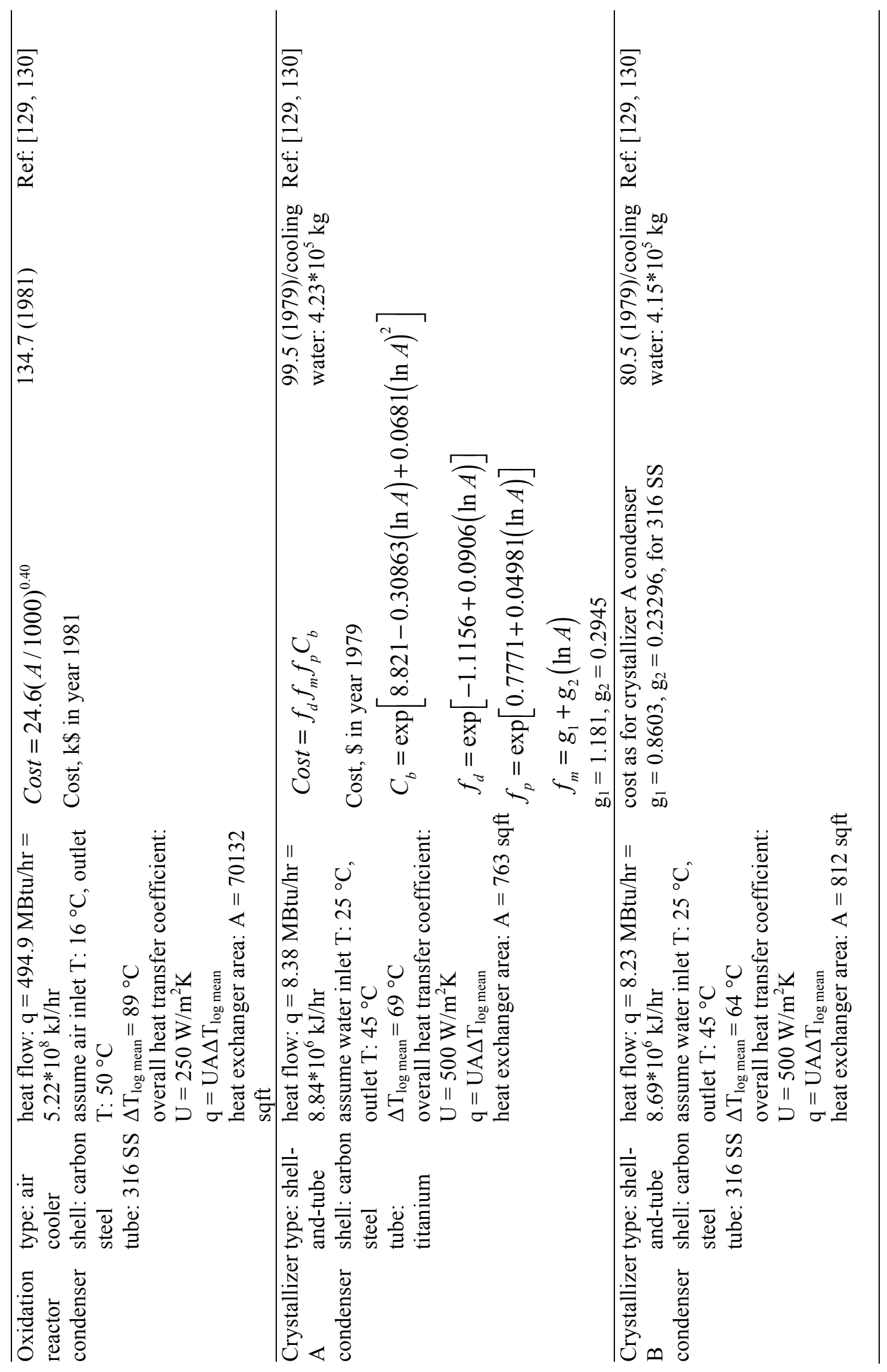




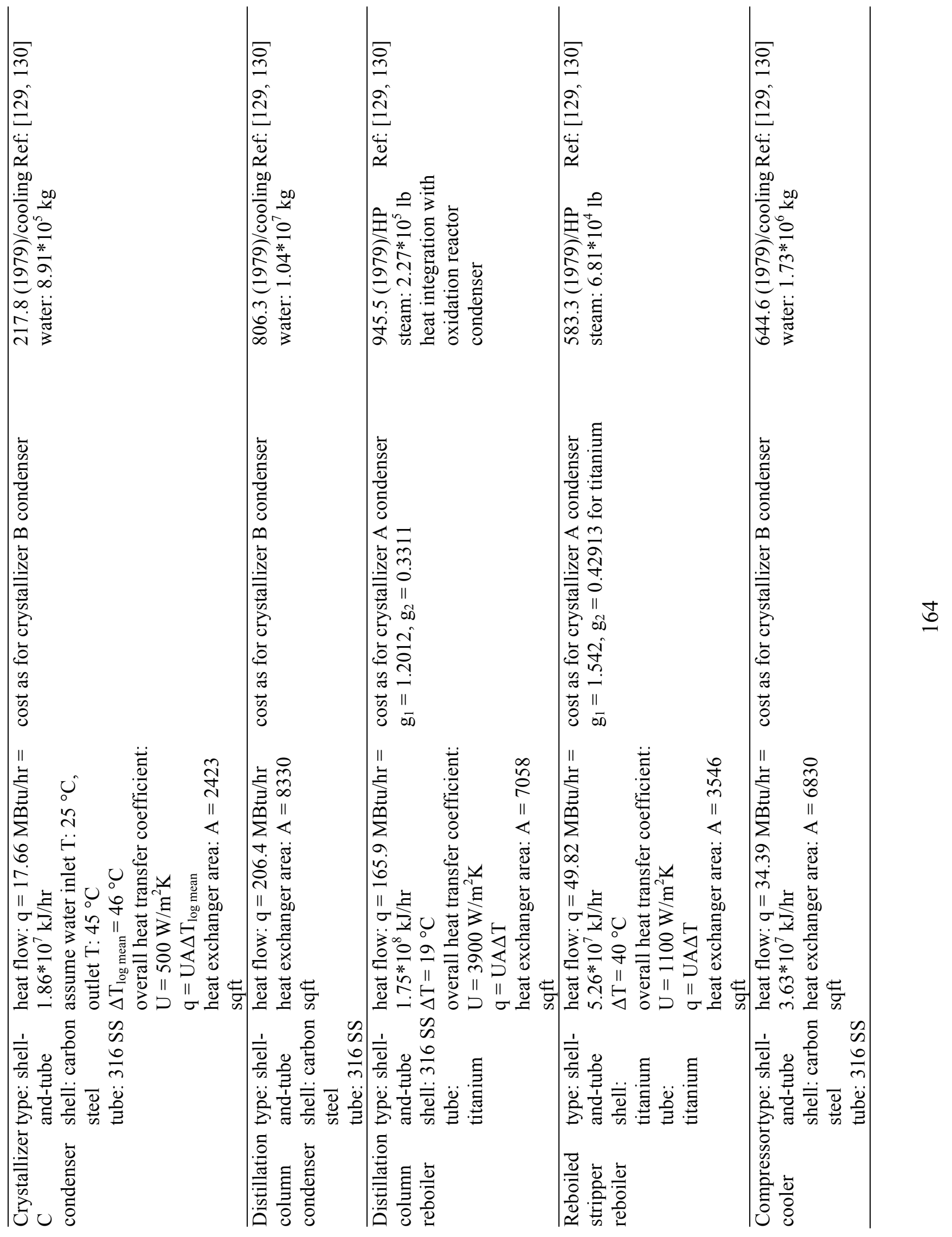




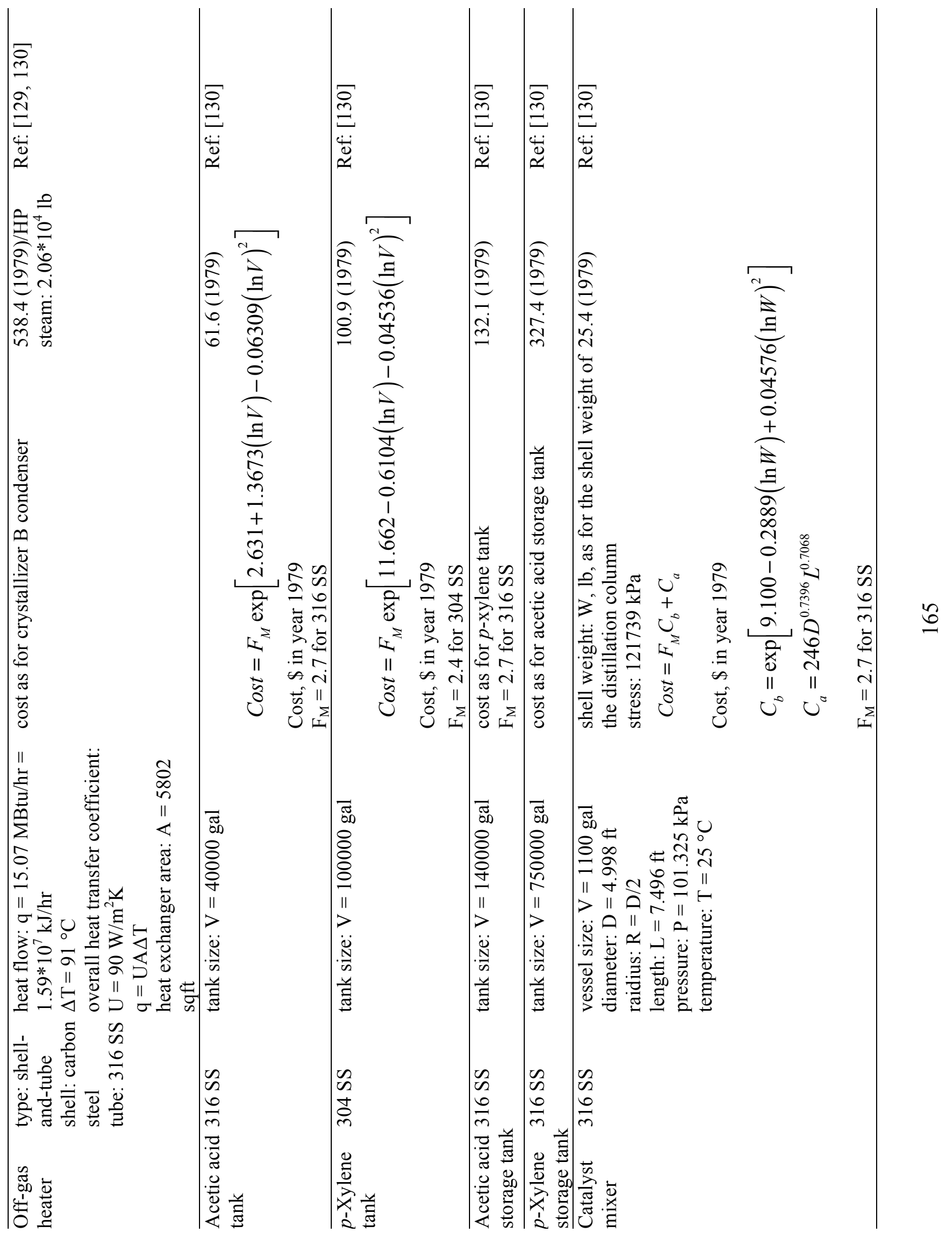




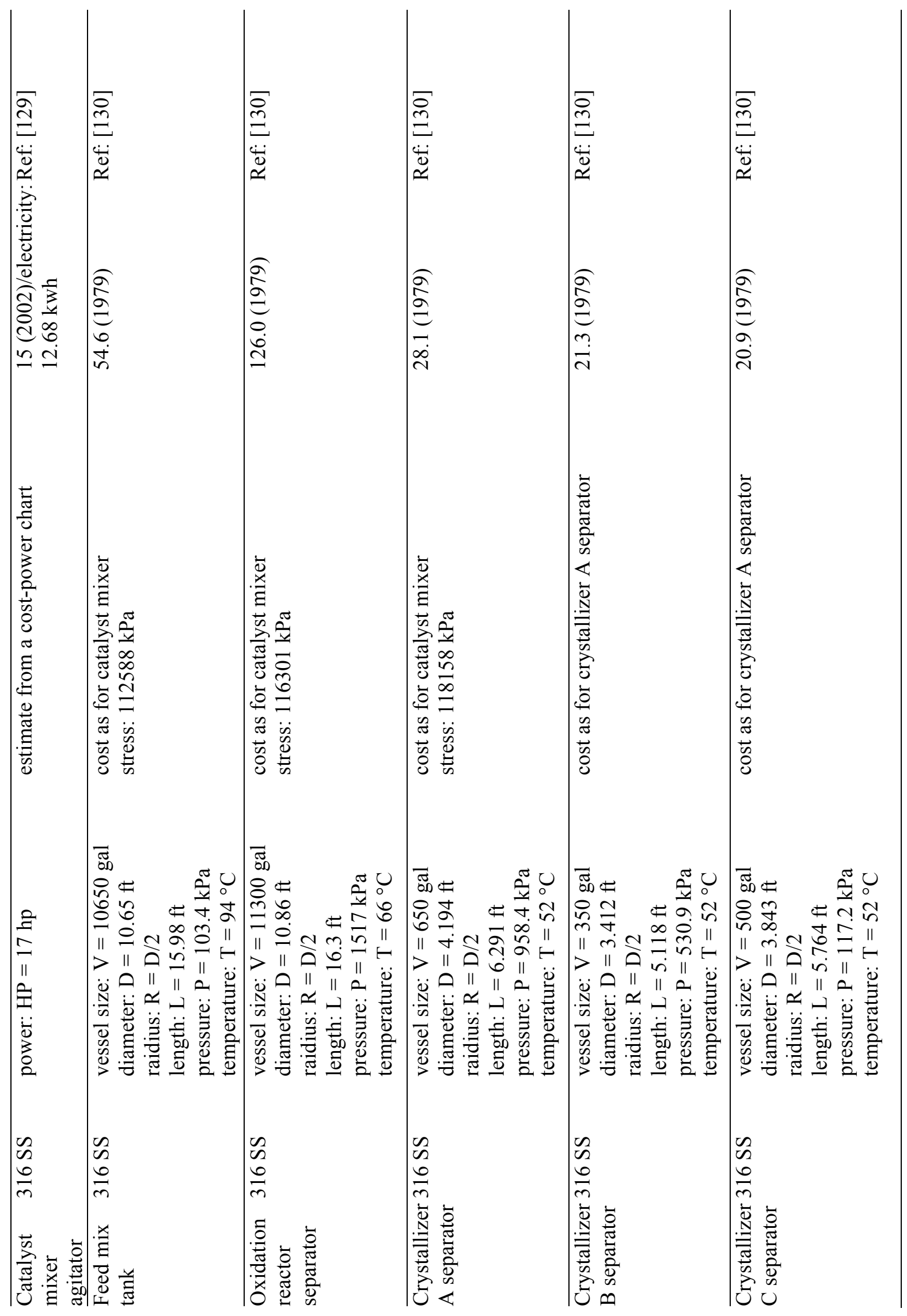




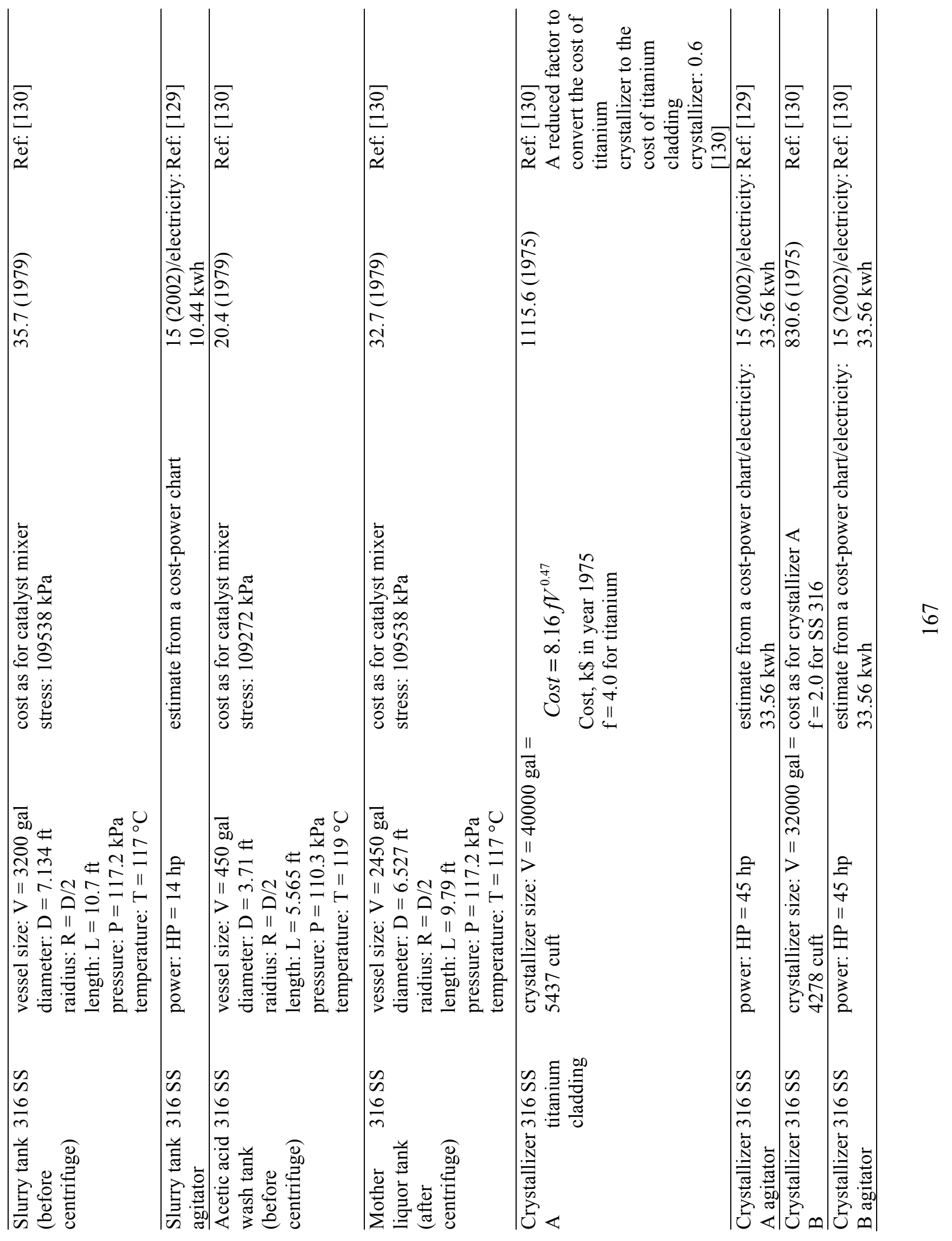




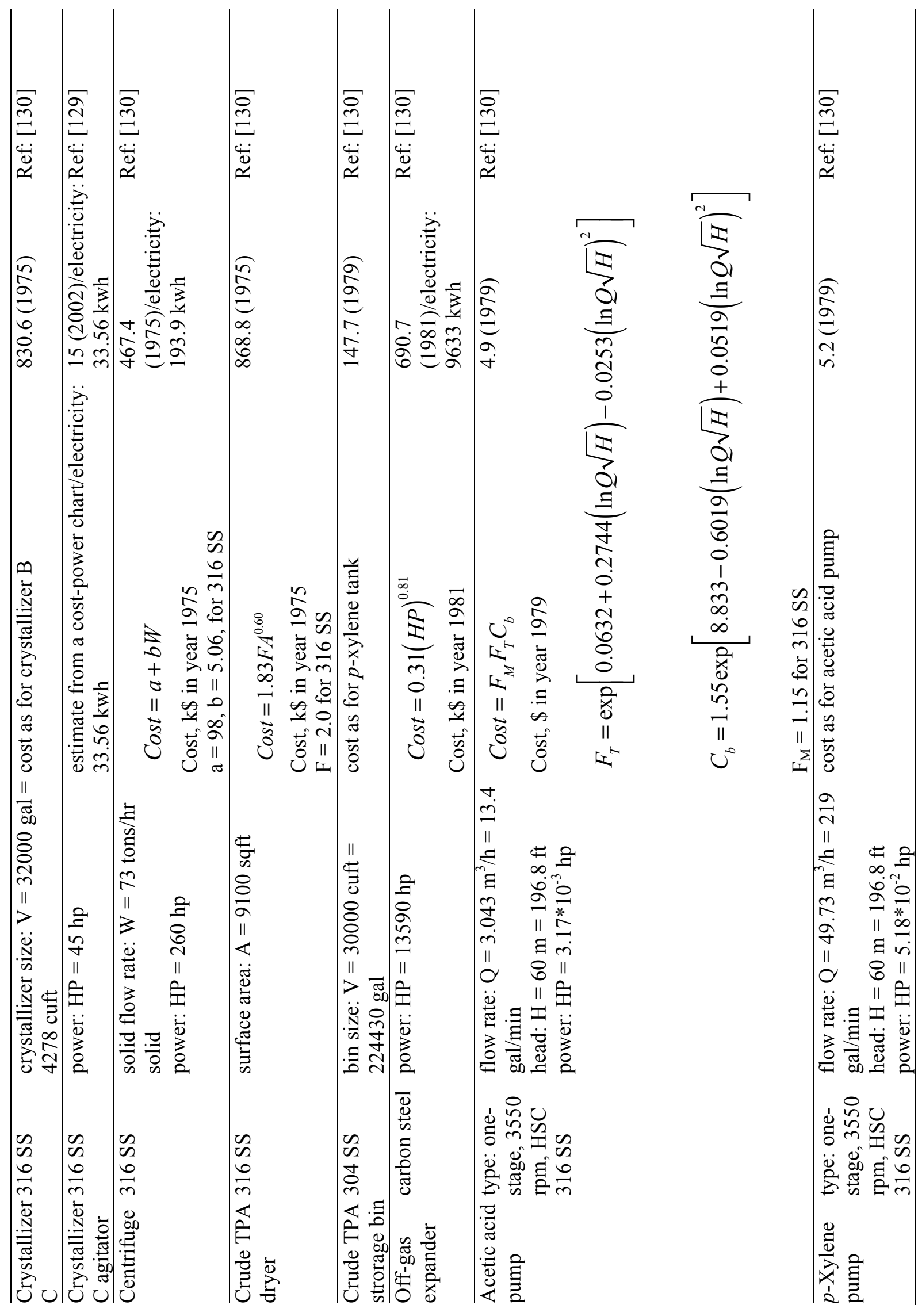




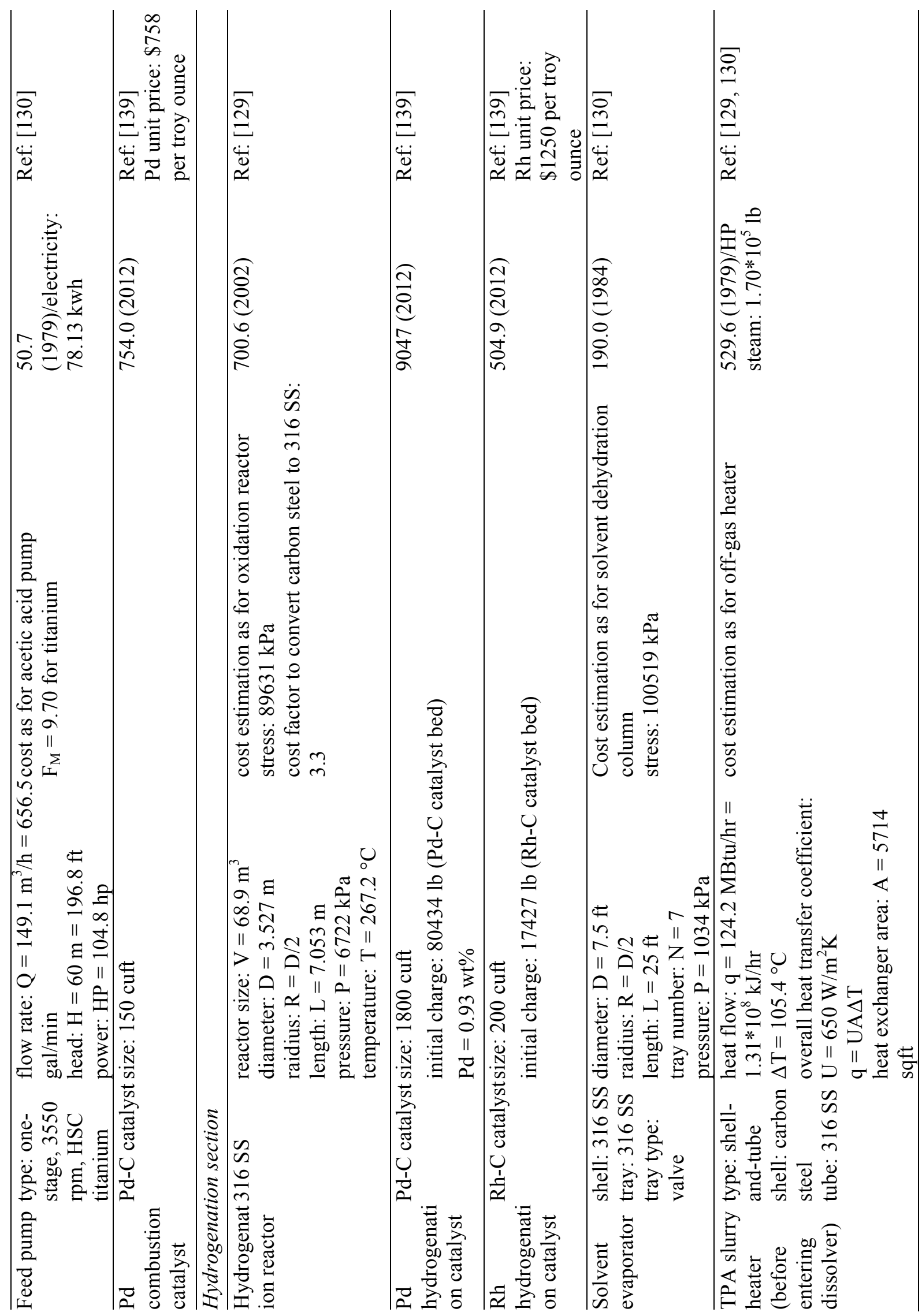

ชำ 


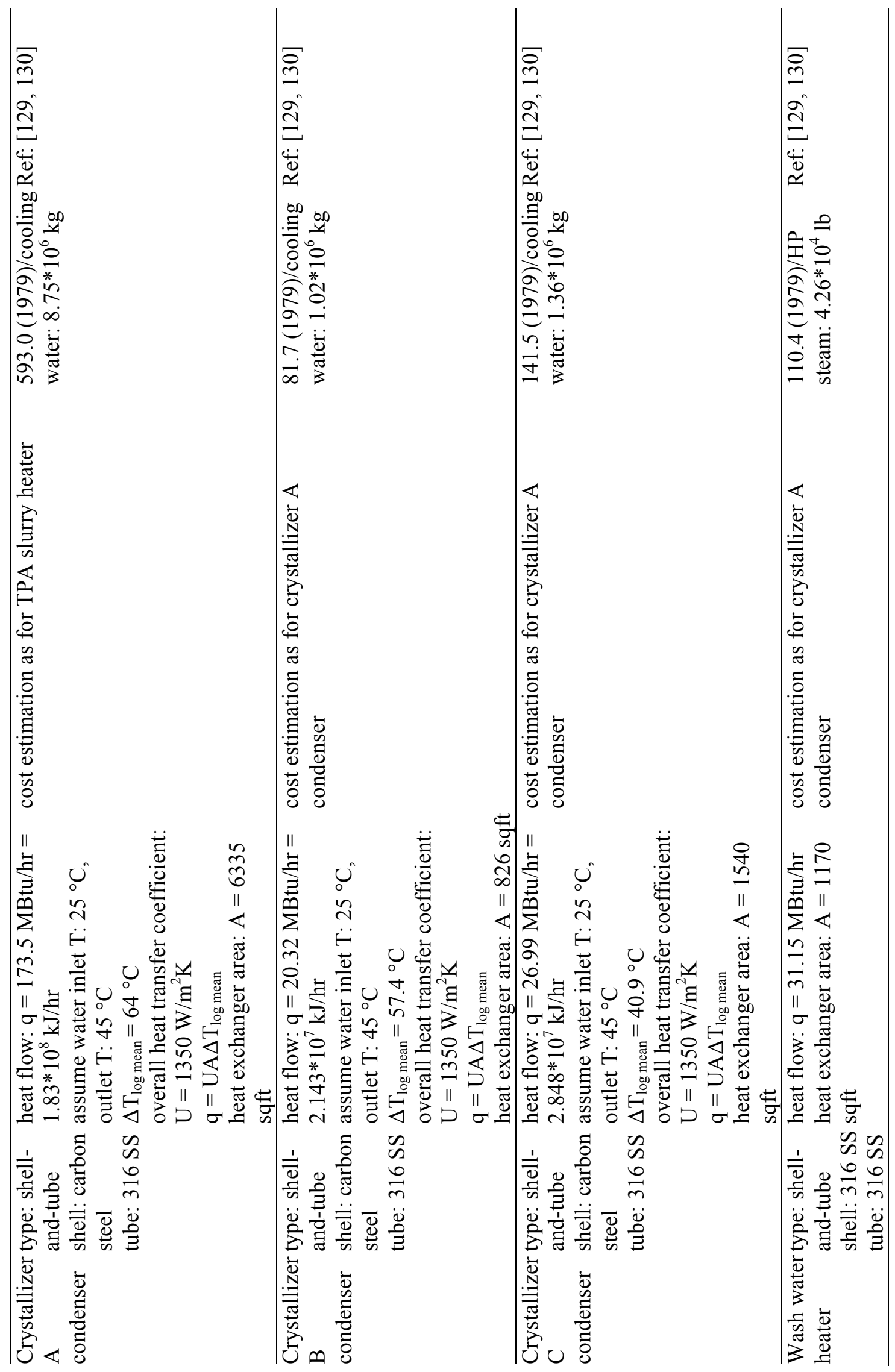




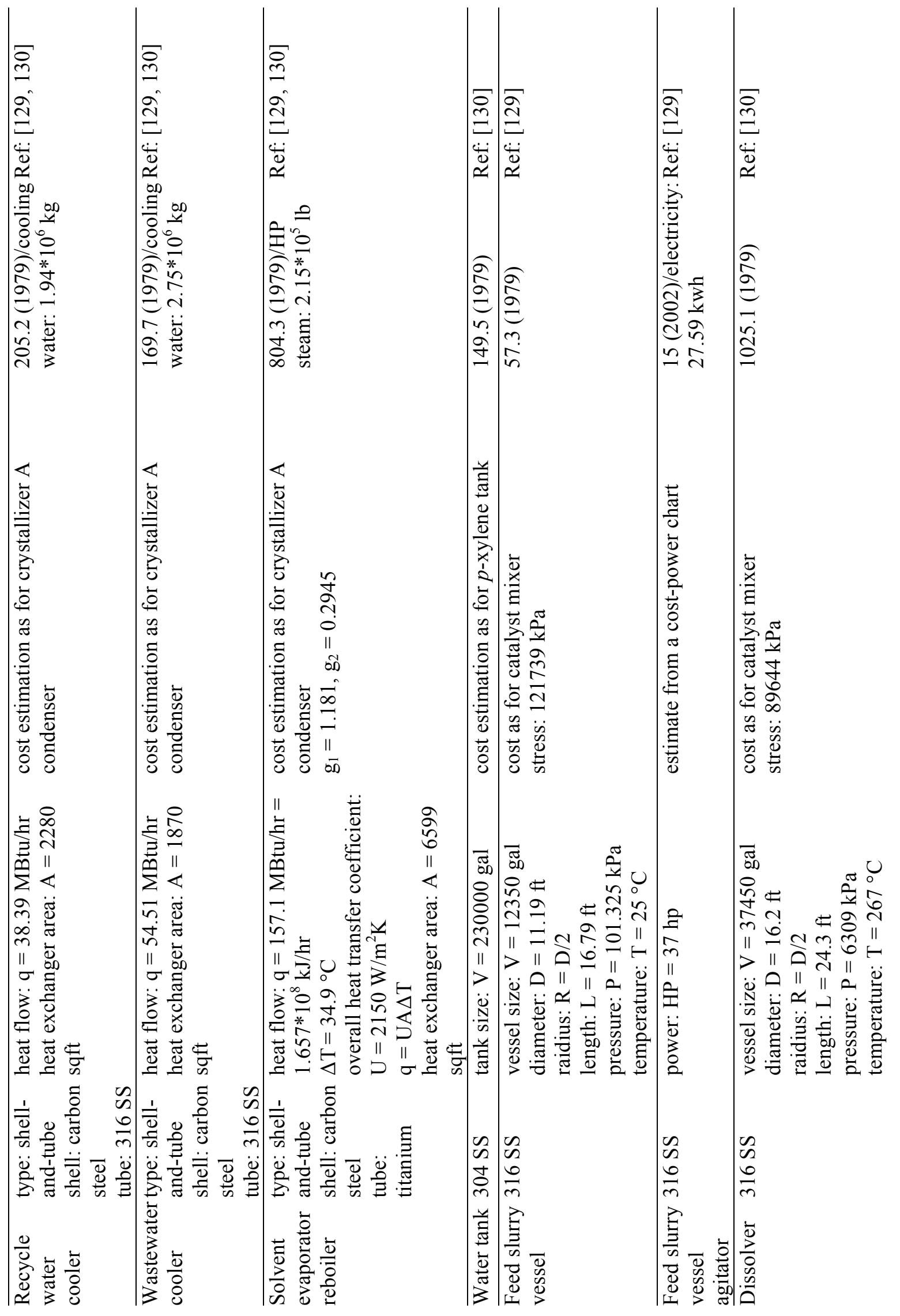




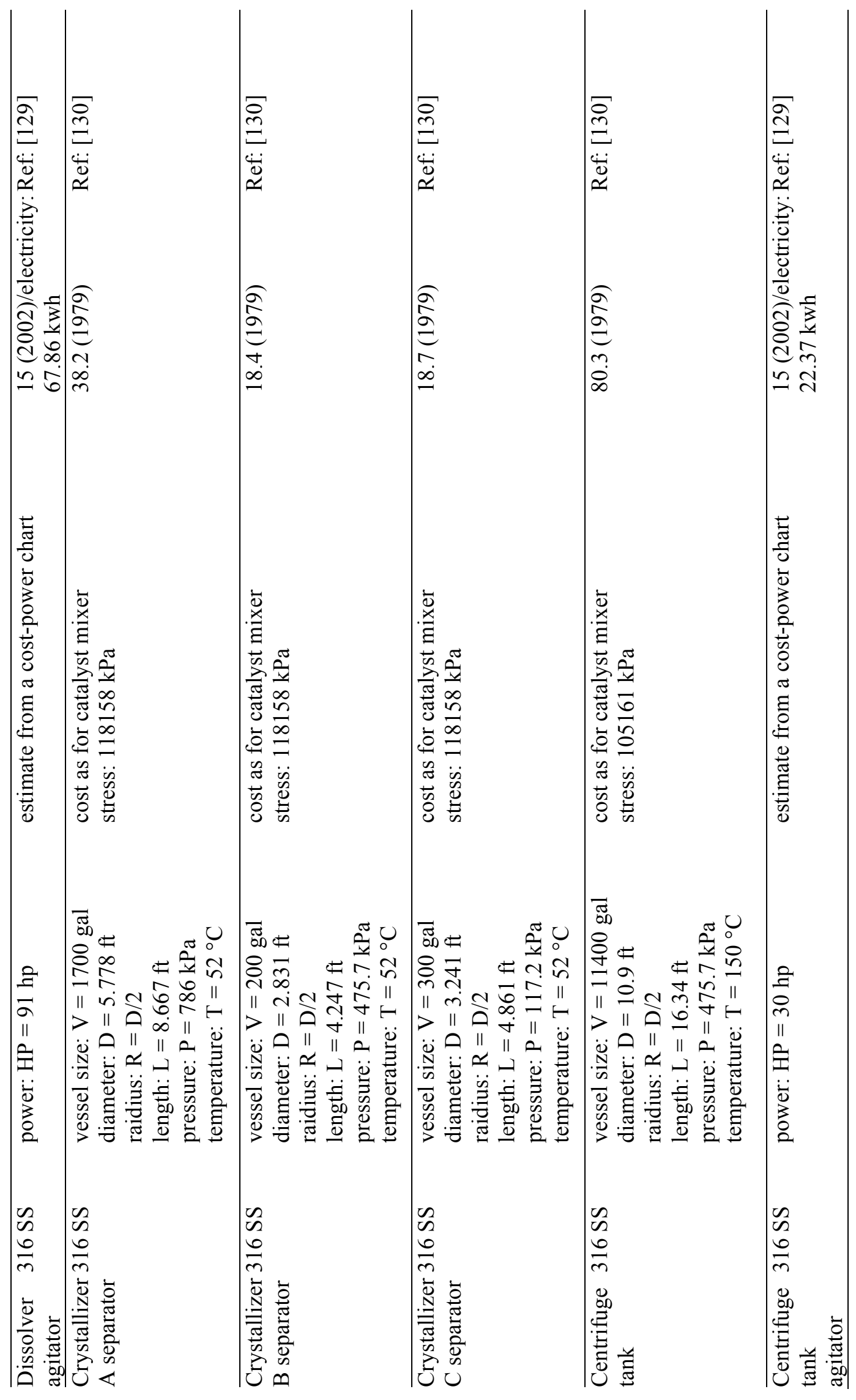




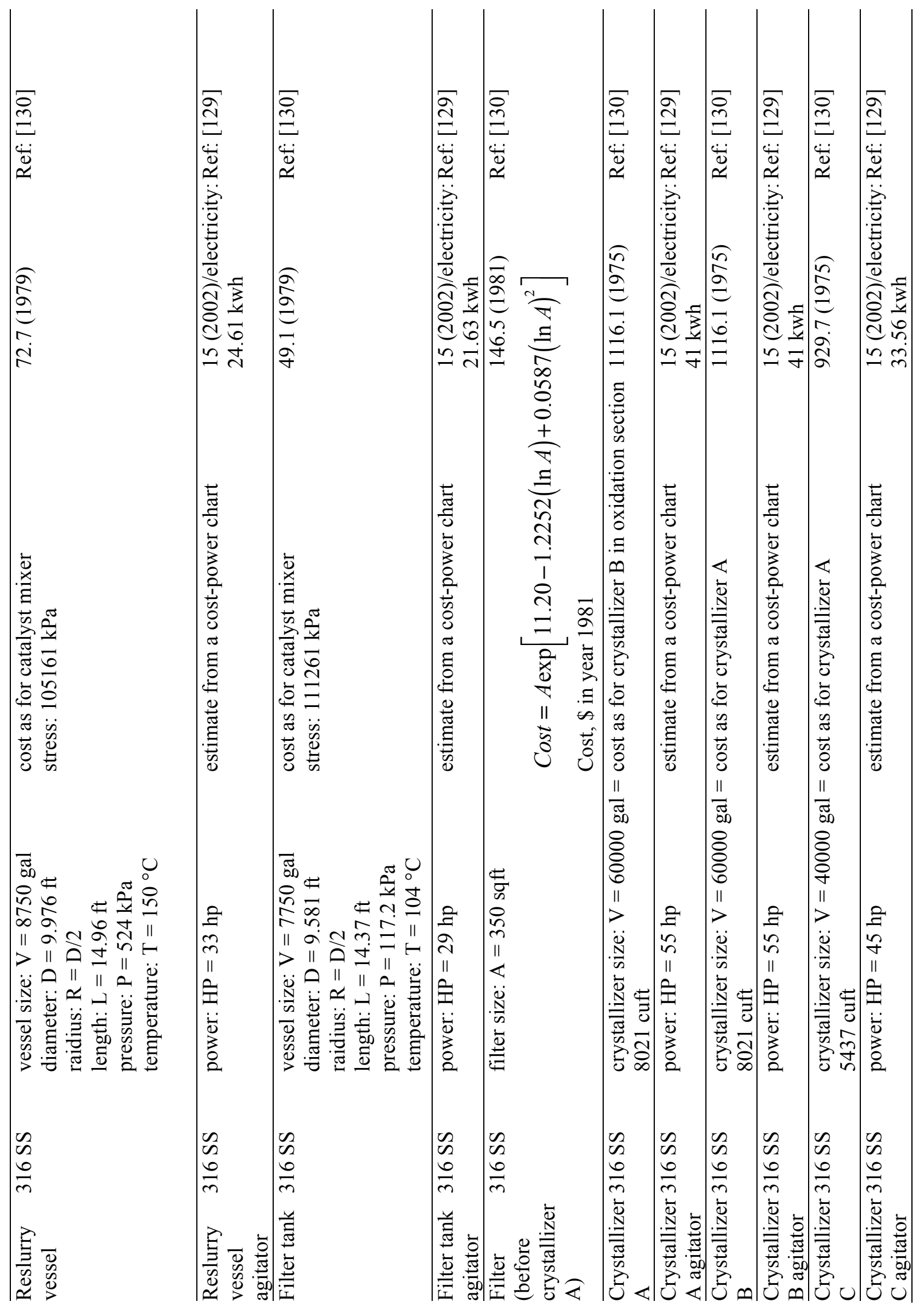

$\stackrel{1}{1}$ 


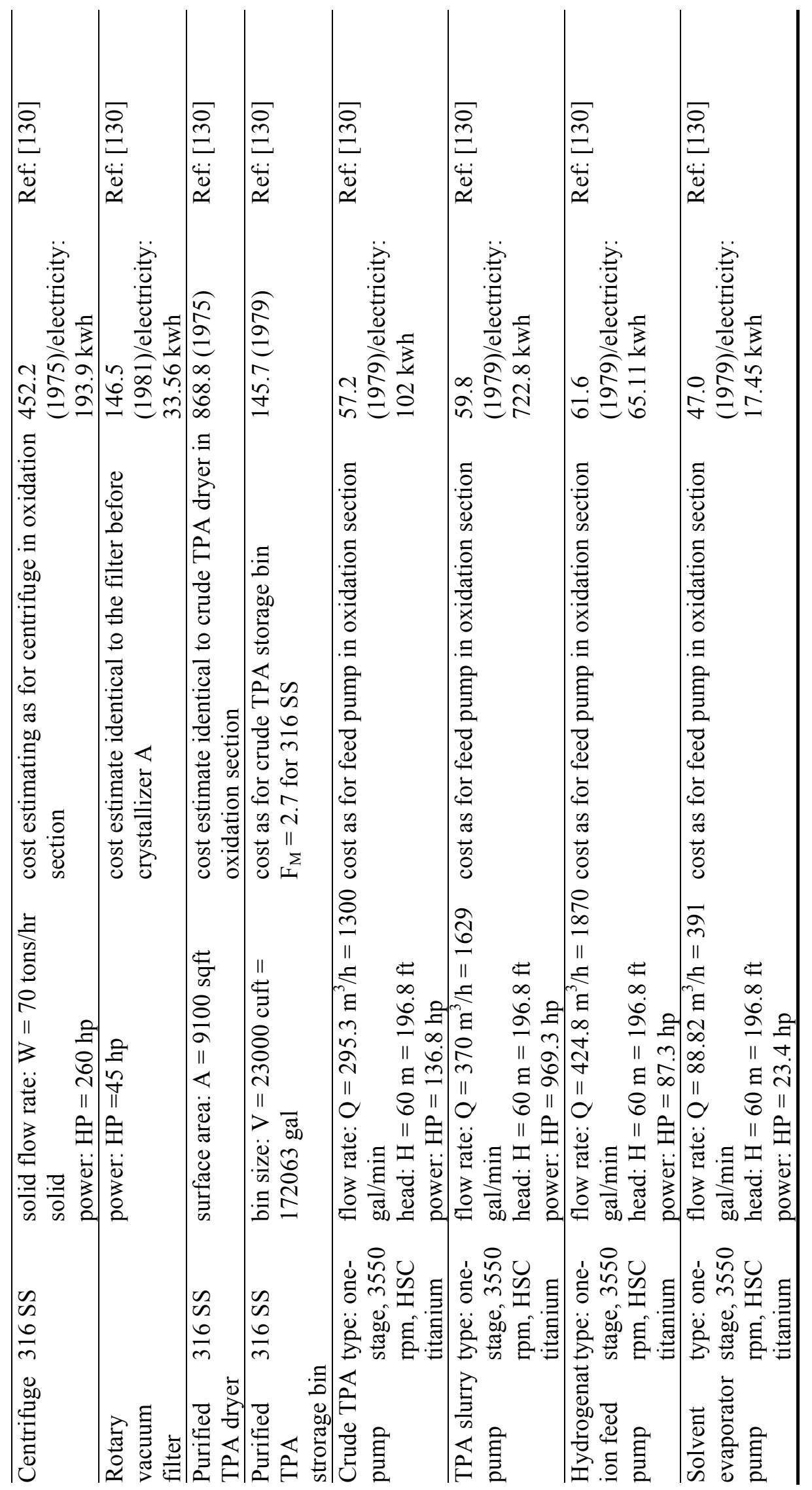




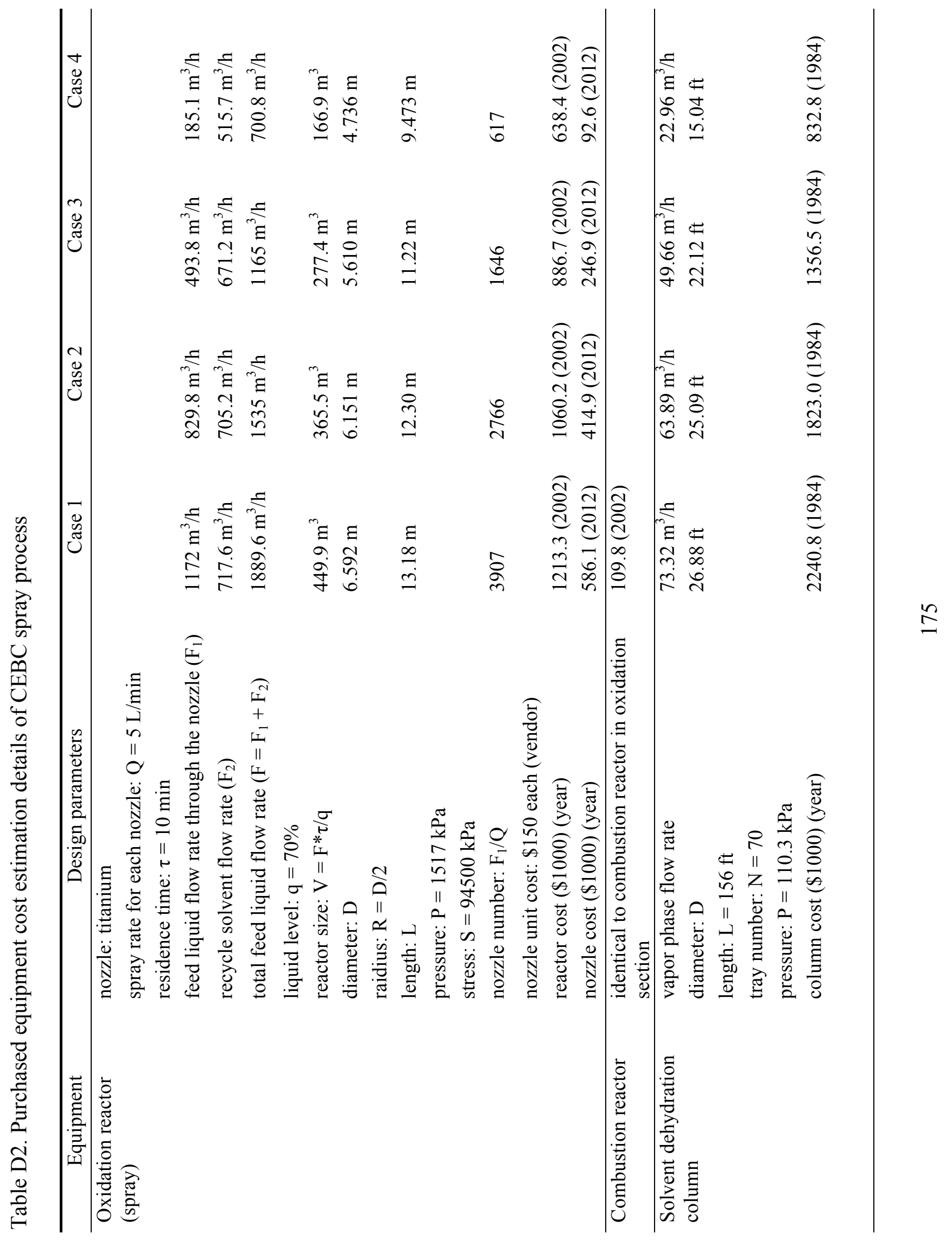




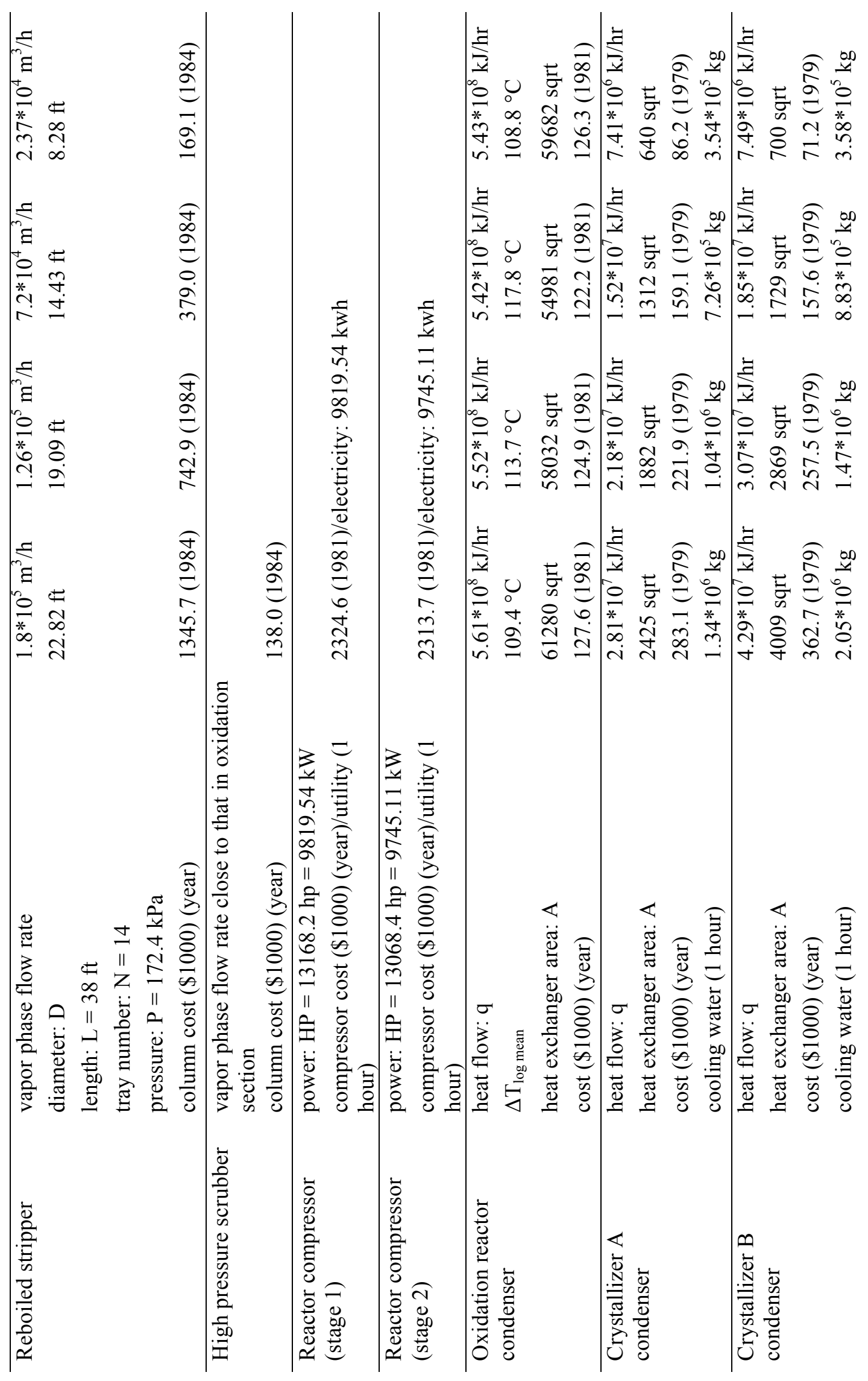




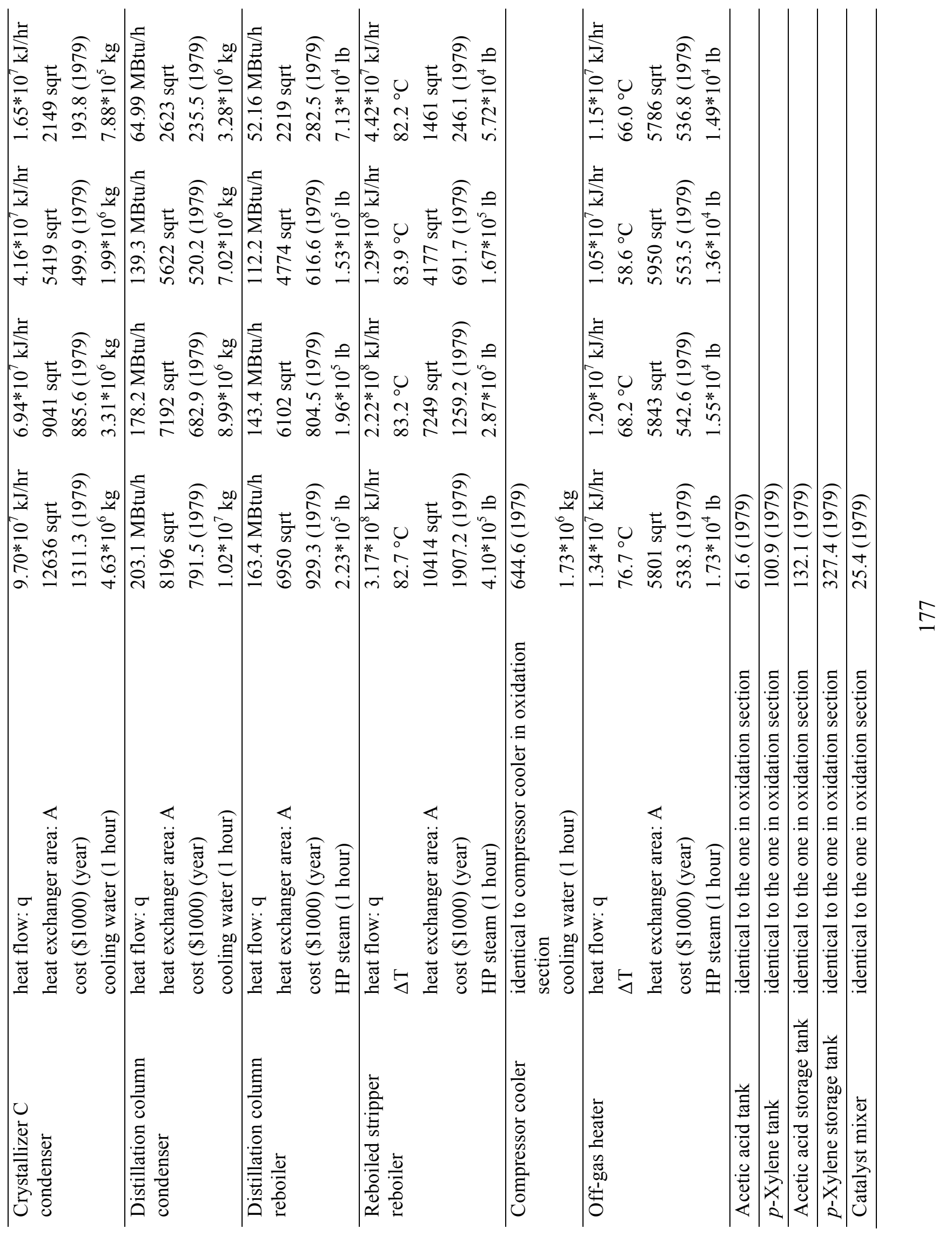




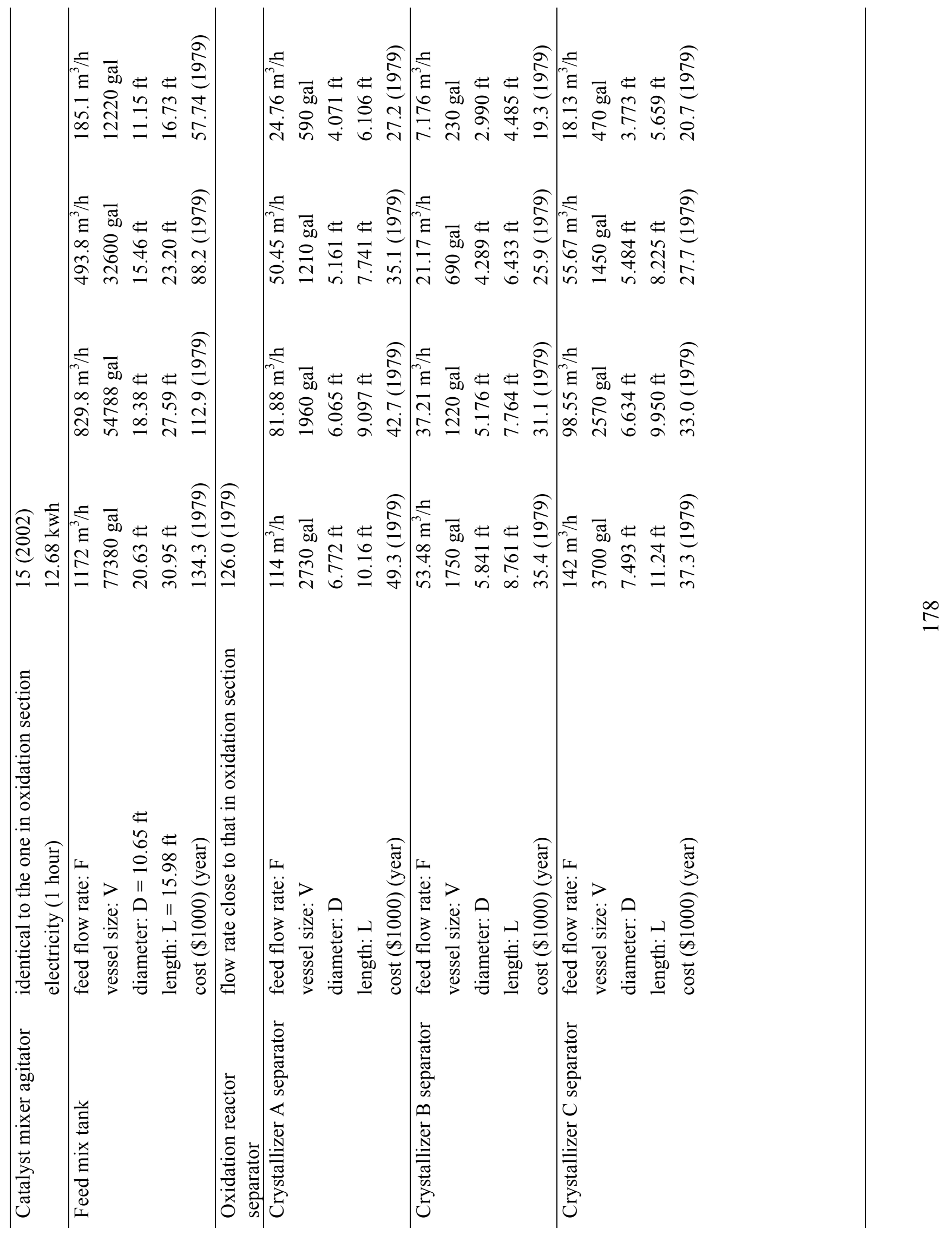




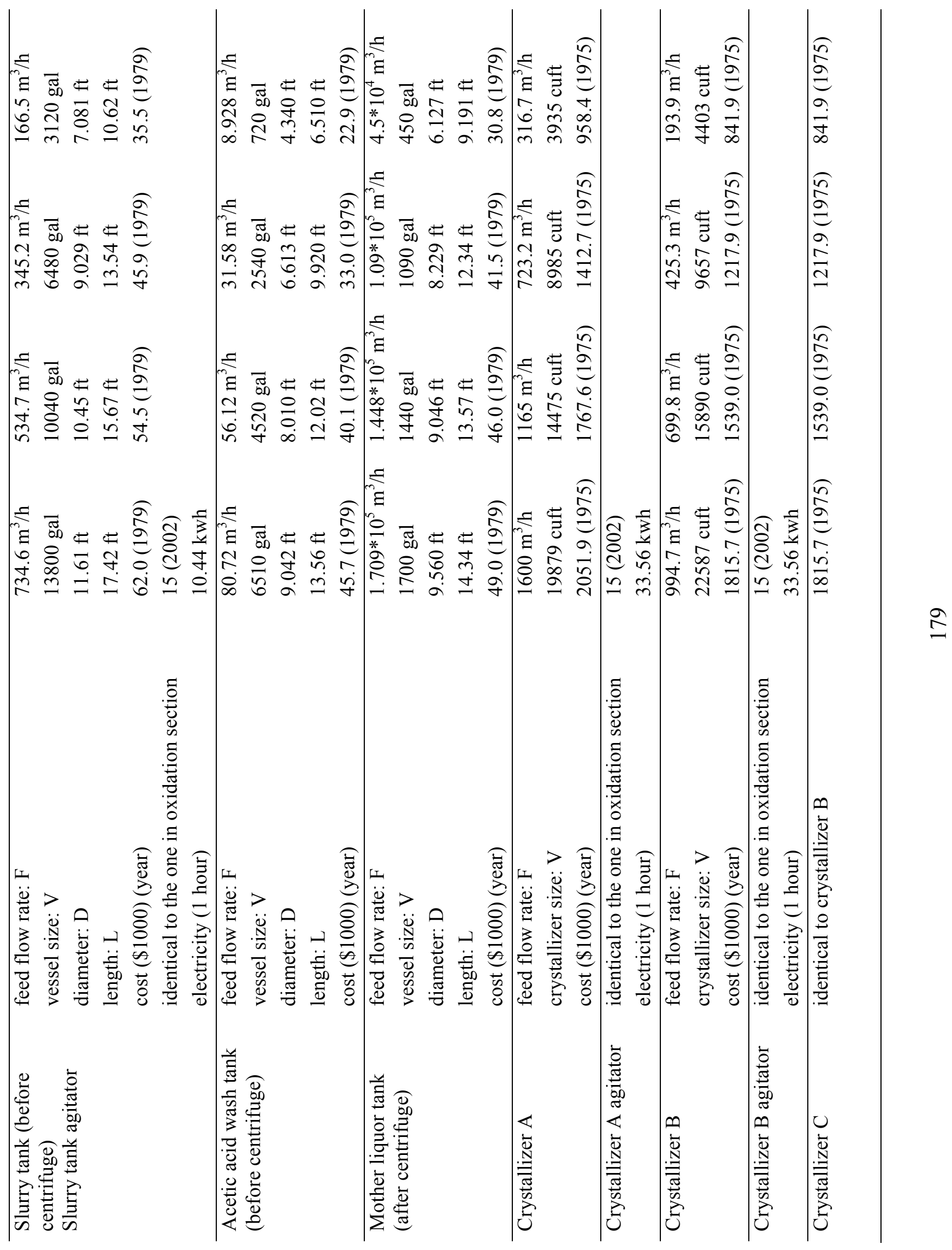




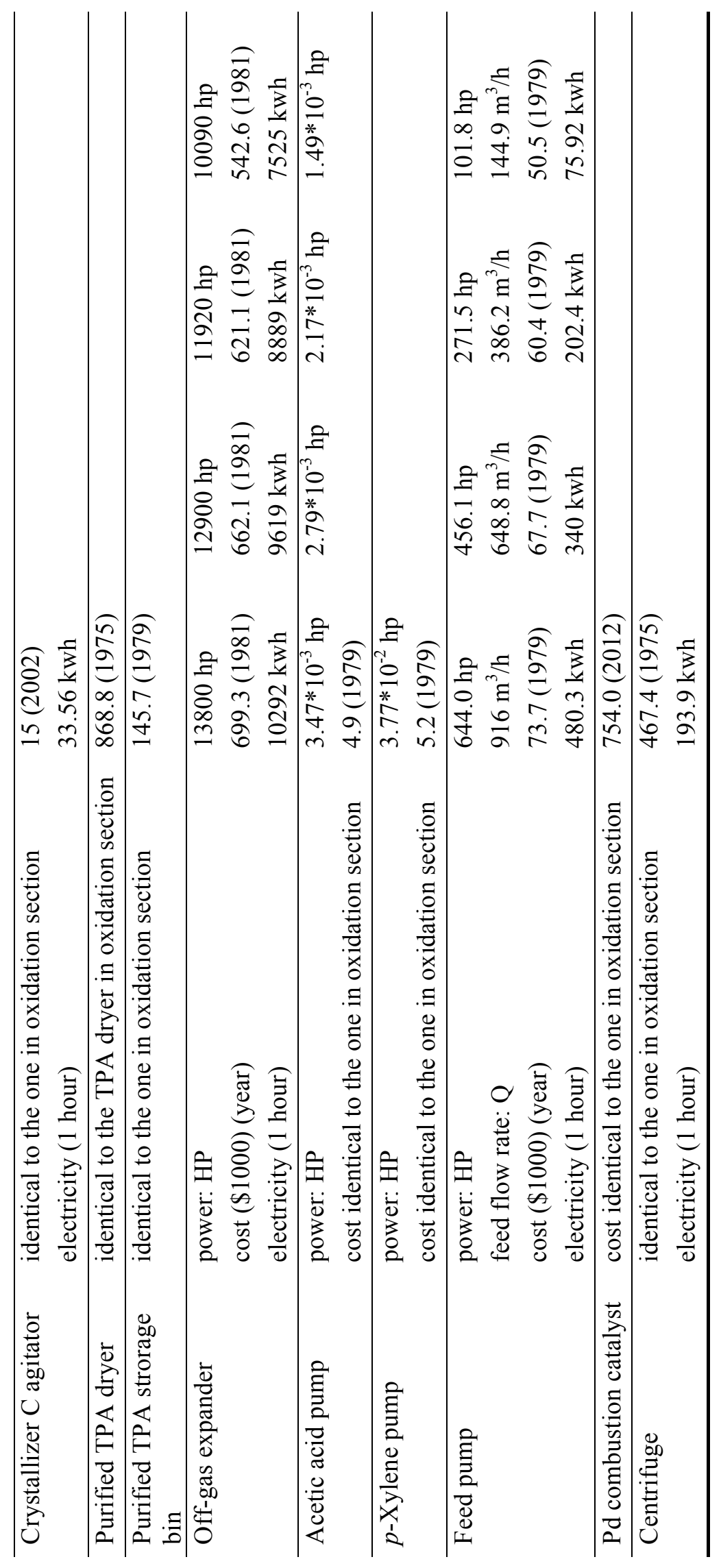




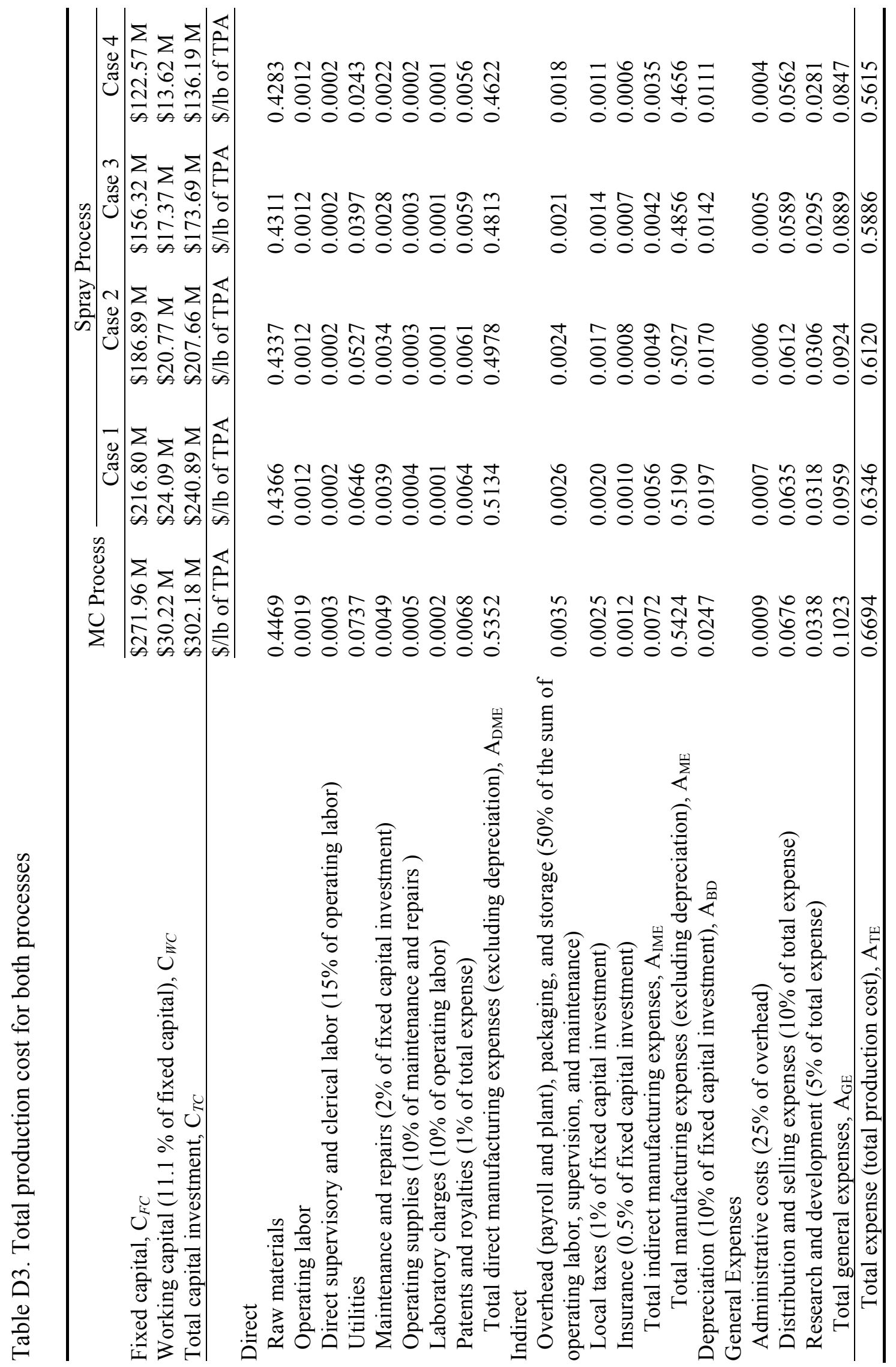




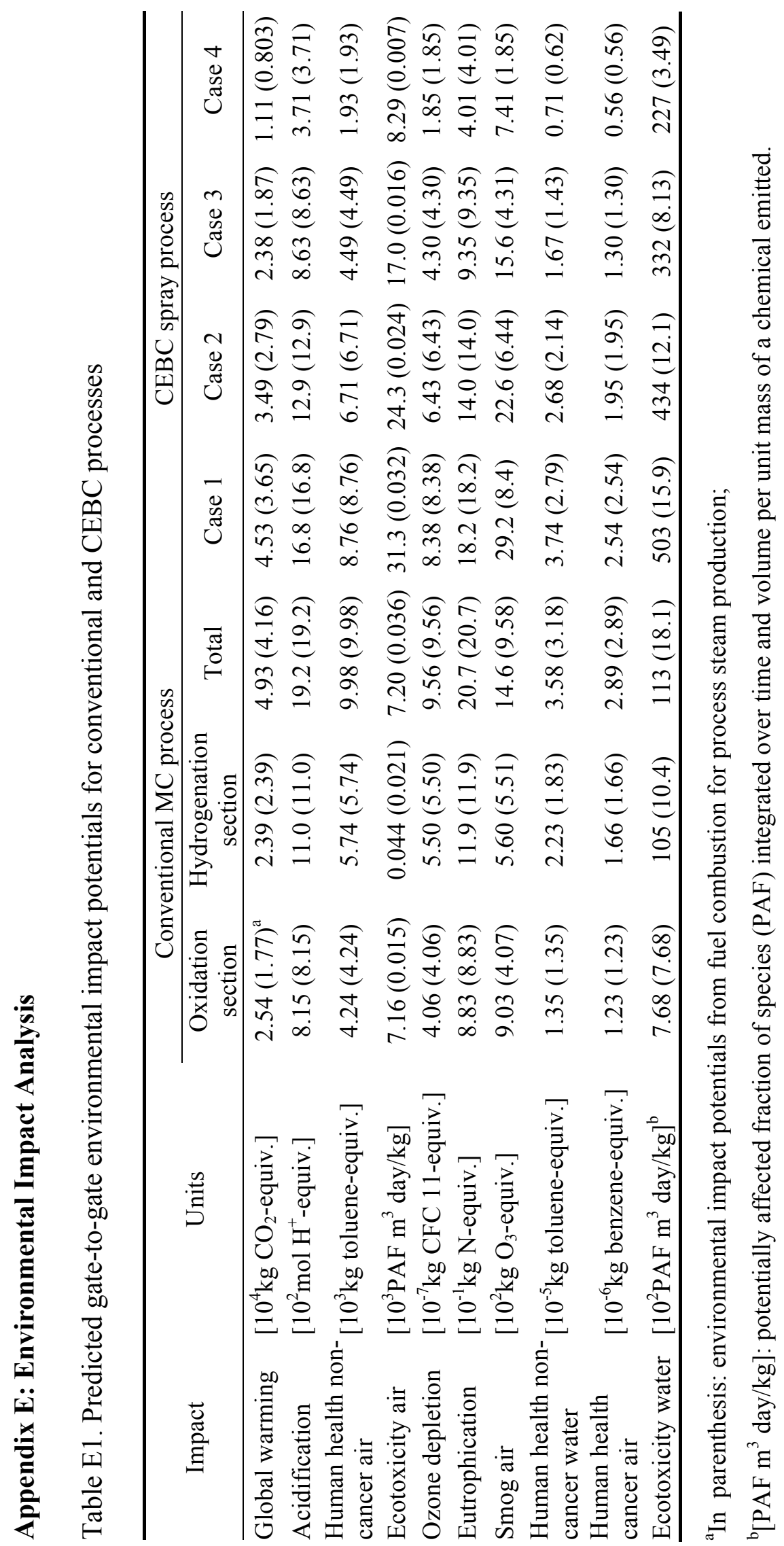




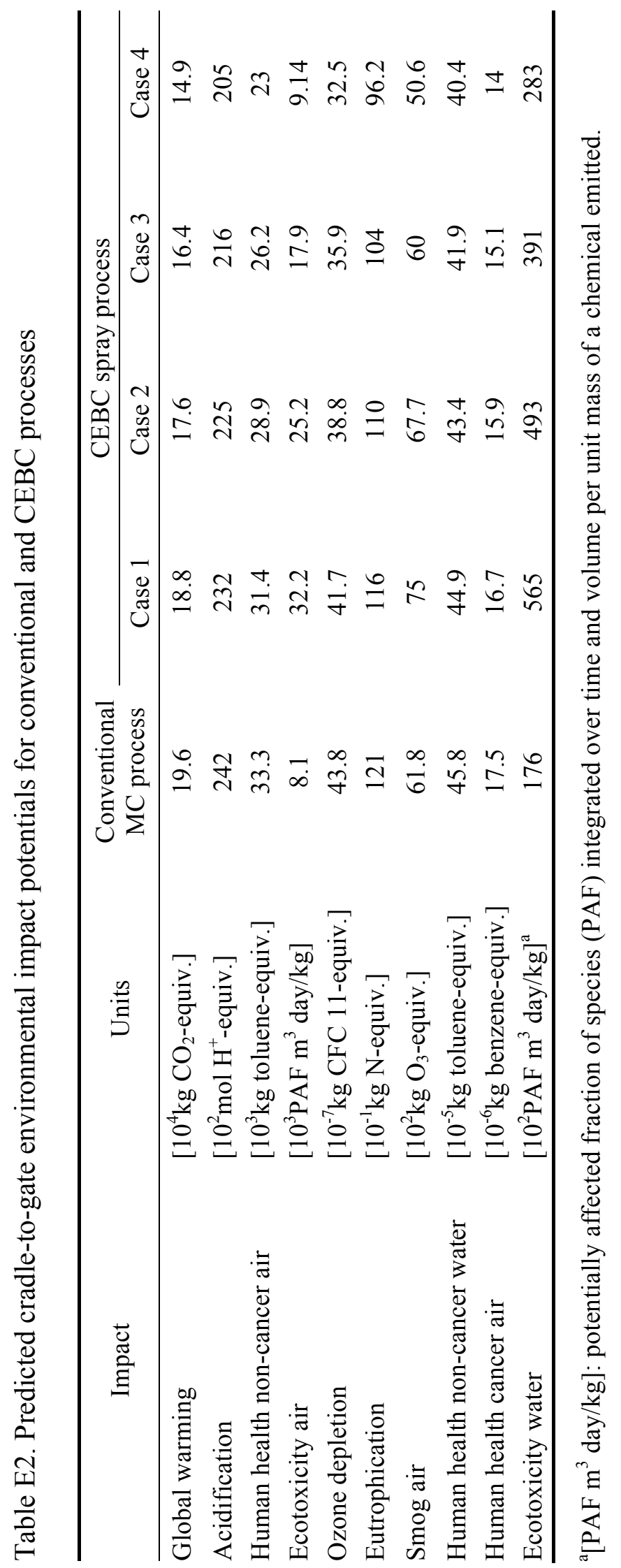

